\title{
Oral anticoagulants as double-edged swords
}

Citation for published version (APA):

van Gorp, R. H. P. (2020). Oral anticoagulants as double-edged swords: balancing between coagulation and calcification. [Doctoral Thesis, Maastricht University]. Gildeprint en Universitaire Pers Maastricht. https://doi.org/10.26481/dis.20200708rg

Document status and date:

Published: 01/01/2020

DOI:

10.26481/dis.20200708rg

Document Version:

Publisher's PDF, also known as Version of record

\section{Please check the document version of this publication:}

- A submitted manuscript is the version of the article upon submission and before peer-review. There can be important differences between the submitted version and the official published version of record.

People interested in the research are advised to contact the author for the final version of the publication, or visit the DOI to the publisher's website.

- The final author version and the galley proof are versions of the publication after peer review.

- The final published version features the final layout of the paper including the volume, issue and page numbers.

Link to publication

\footnotetext{
General rights rights.

- You may freely distribute the URL identifying the publication in the public portal. please follow below link for the End User Agreement:

www.umlib.nl/taverne-license

Take down policy

If you believe that this document breaches copyright please contact us at:

repository@maastrichtuniversity.nl

providing details and we will investigate your claim.
}

Copyright and moral rights for the publications made accessible in the public portal are retained by the authors and/or other copyright owners and it is a condition of accessing publications that users recognise and abide by the legal requirements associated with these

- Users may download and print one copy of any publication from the public portal for the purpose of private study or research.

- You may not further distribute the material or use it for any profit-making activity or commercial gain

If the publication is distributed under the terms of Article $25 \mathrm{fa}$ of the Dutch Copyright Act, indicated by the "Taverne" license above, 


\title{
Oral anticoagulants as double-edged swords:
}

\section{balancing between coagulation and calcification}

\author{
RH van Gorp
}


This project has been financially supported by the Norwegian Research Council under the industrial Ph.D program.

Cover design: $\quad$ Lucie van den Driessche, lucie.studio

Layout: $\quad$ Rick van Gorp

Printed by: $\quad$ Gildeprint

ISBN: $\quad 9789464022025$

(C) Copyright Rick H. van Gorp, Maastricht 2020.

All rights reserved. No part of this thesis may be reproduced, stored in a retrieval system or transmitted in any form or means, without the permission in writing from the author. 


\title{
Oral anticoagulants as double-edged swords: balancing between coagulation and calcification
}

\author{
PROEFSCHRIFT
}

Ter verkrijging van de graad van doctor aan de Universiteit Maastricht, op gezag van de Rector Magnificus, Prof. Dr. Rianne M. Letschert, volgens het besluit van het College van Decanen,

in het openbaar te verdedigen op woensdag 8 juli 2020 om 16:00 uur.

door

Rick Henricus Petrus van Gorp

Geboren op 3 maart 1991 te Oosterhout, Nederland 


\section{Promotoren}

Prof. Dr. L.J. Schurgers

Prof. Dr. C.P.M. Reutelingsperger

Beoordelingscommissie

Prof. Dr. A.A Kroon (voorzitter)

Prof. Dr. S. Middeldorp (AMC, Amsterdam)

Prof. Dr. M.J. Post

Prof. Dr. E.A.L. Biessen

Dr. H.H. Boersma (UMCG, Groningen) 


\section{Content}

$\begin{array}{lll}\text { Chapter } 1 \text { General introduction } & 7\end{array}$

Chapter 2 New Insights into the Pros and Cons of the Clinical Use of 17 Vitamin K Antagonists (VKAs) Versus Direct Oral Anticoagulants (DOACs)

Chapter 3 Vitamin K antagonist induces calcification and atherosclerotic plaque progression resulting in increased hypercoagulability

Chapter 4 Vitamin K inhibits vascular smooth muscle cell induced calcification via reduced oxidative stress and extracellular vesicle secretion

Chapter 5 Off-target effects of oral anticoagulants - Vascular effects of vitamin $\mathrm{K}$ antagonist and non-vitamin $\mathrm{K}$ antagonist oral anticoagulants

Chapter 6 Reactive oxygen-forming Nox5 links vascular smooth muscle cell phenotypic switching and extracellular vesicle-mediated-vascular calcification

Chapter 7 General discussion

Addenum Summary

Nederlandse samenvatting

Valorisation

About the author

Publications

Dankwoord 


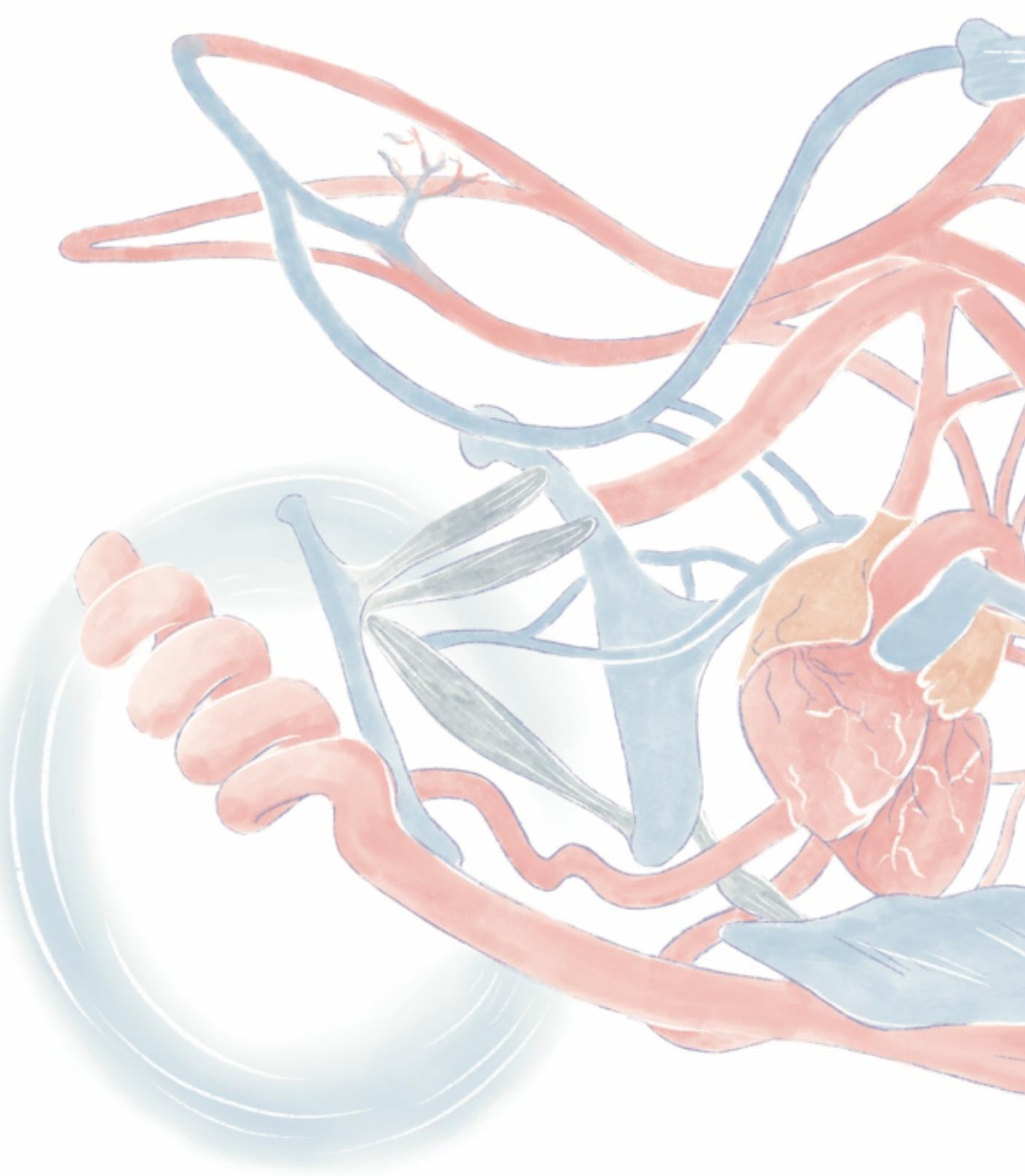




\section{Chapter 1}

General introduction 


\section{Cardiovascular disease}

Cardiovascular diseases (CVDs) are pathologies involving heart and vessels of the body. In 2017, CVD was the number one cause of death globally (WHO, May 2017). Within CVD mortality, $85 \%$ was because of a heart attack or stroke. Both heart attack (myocardial infarction) and stroke (brain infarction) can be the result of a ruptured atherosclerotic plaque. Atherosclerosis is the development of lesions in the intimal space of the arterial wall, ultimately hampering blood flow or causing thrombosis (1). Plaques develop at sites with high shear stress of which the branches in coronary arteries, carotid arteries and aorta are the most common sites (1). With time, atherosclerotic lesions progress from an intermediate lesion to an advanced atherosclerotic plaque (atheroma). Advanced atherosclerotic plaques can be divided into fibroatheroma, calcific, fibrous or thrombotic lesions (Table 1). Rupture of an atherosclerotic plaque potentially exposes the thrombotic core of the atherosclerotic plaque triggering thrombosis (also known as atherothrombosis) and subsequently leading to myocardial infarction or stroke (1).

Table 1. Atherosclerotic lesion progression (adapted from $(2,3)$ ).

\begin{tabular}{|c|c|c|}
\hline $\begin{array}{l}\text { Atherosclerotic plaque } \\
\text { classification }\end{array}$ & & Histological presentation \\
\hline Type I: & \multicolumn{2}{|c|}{ Initial lesion, with foam cells } \\
\hline Type II: & \multicolumn{2}{|c|}{ Early lesion, fatty streak, with multiple foam cell layers / microcalcification } \\
\hline Type III: & \multicolumn{2}{|c|}{ Intermediate lesions, extracellular lipid pools / microcalcification } \\
\hline Type IV: & \multicolumn{2}{|c|}{ atheroma, confluent extracellular lipid core formation } \\
\hline Type V: & \multicolumn{2}{|c|}{ Advanced lesion, fibroatheroma, fibromuscular tissue layers produced } \\
\hline Type VI: & \multicolumn{2}{|c|}{ Advanced lesion, complicated lesion, surface defect, hematoma, thrombosis } \\
\hline \multirow{2}{*}{ Type VII: } & Advanced lesion & $\begin{array}{l}\text { Microcalcification, calcium deposits }<50 \mu \mathrm{m} \text {, increases } \\
\text { chance of plaque rupture }\end{array}$ \\
\hline & Calcified plaques & $\begin{array}{l}\text { Macrocalcification, calcium deposits } \geq 50 \mu \mathrm{m}, \\
\text { stabilization of atherosclerotic plaque }\end{array}$ \\
\hline Type VIII: & \multicolumn{2}{|c|}{$\begin{array}{l}\text { Advanced lesion, fibrotic plaque without lipid core, fibrous tissue changes } \\
\text { predominates }\end{array}$} \\
\hline
\end{tabular}

\section{Coagulation}

Physiologically, coagulation prevents excessive blood loss after injury. However, risk factors including genetic predisposition, therapeutics and life style can result in increased coagulation potential with risk of thrombosis independent of vascular injury (4). Thrombosis can be classified as venous thromboembolism and arterial thrombosis. Venous thromboembolism consists mainly of deep vein thrombosis, which can induce pulmonary embolism when the blood clot breaks free. Arterial thrombosis is usually the consequence of atherosclerotic plaque erosion or rupture $(5,6)$. Patients experiencing thrombosis are prescribed anticoagulant therapy to reduce risk of recurrent thrombosis. Most used long-term anticoagulation drugs are vitamin $\mathrm{K}$ antagonists (VKA). Vitamin $\mathrm{K}$ is a cofactor for the posttranslational carboxylation and is recycled via the vitamin K-epoxide-reductase (VKOR) 
thereby efficiently used to activate vitamin K dependent proteins (VKDP) (7). VKA inhibit the VKOR enzyme thereby blocking re-cycling and thus re-use of vitamin $\mathrm{K}$, subsequently limiting carboxylation of VKDP (8). Use of VKA results in undercarboxylated vitamin K dependent coagulation factors II, VII, IX and X thereby inducing reduced coagulation. Since only recycling of vitamin $\mathrm{K}$ is limited, vitamin $\mathrm{K}$ supplementation counteracts VKA treatment. Recently, anticoagulant therapeutics targeting specifically activated coagulation factor IIa (thrombin) or Xa have been approved and are now being used in the clinic $(8,9)$. These so called direct non-vitamin $\mathrm{K}$ oral anticoagulants (NOAC) inhibit the activated coagulation factors by binding to their active sites (8).

In addition to their role in the coagulation cascade, thrombin and factor Xa have been implicated in the development of atherosclerosis via protease activated receptor (PAR) signaling $(10,11)$. Upregulation or downregulation of thrombin has been shown to aggravate or reduce atherosclerosis severity, respectively in animal models $(9,12)$. Thus, blocking thrombin and factor Xa activity using NOACs may influence atherosclerosis development. VKA on the other hand is not expected to influence atherogenesis via PARs since uncarboxylated coagulation factors retain their ability to activate PAR signaling (9). However, VKA affects atherogenesis differently, namely by reducing the carboxylation of extrahepatic VKDP, especially the vascular calcification inhibitor matrix Gla protein (MGP) (13). VKA has been shown to increase vascular calcification in the $A p o e^{-/-}$mouse model of atherosclerosis (14). VKA use is associated with a progressive increase of vascular calcification in patients $(14,15)$.

\section{Vascular calcification}

Vascular calcification present in any vascular bed increases chance of cardiovascular events by some 3 to 4 -fold (16). Additionally, coronary artery calcification is a predictor for overall plaque burden and an independent risk factor for cardiovascular events that improves standard risk assessment $(17,18,19)$. Moreover, progression of calcification over time has been associated with a 17-fold increased risk of cardiovascular events (20). Calcification can occur in the medial layer (also known as Monckebergs sclerosis) or in the intimal layer of the vasculature (Figure 1). Medial and intimal calcification are two different entities (21). Medial calcification is associated with aging, chronic kidney disease (CKD) and diabetes mellitus. Intimal calcification is associated with hypercholesterolemia and inflammation (22). While both medial and intimal calcification share common mechanistic pathways, such as loss of inhibitors, they have different clinical consequences. Therefore, it is important to distinguish between the two types of calcification (21). Medial calcification presents as railtracking deposits along the vasculature and affects vascular stiffness. Intimal calcification presents as spotty or sheet like calcification and is linked to plaque instability. Further to this, both forms of calcification can be divided in micro- and macrocalcification based on size of the calcification (Figure 1, Table 2). 


\section{Chapter 1}
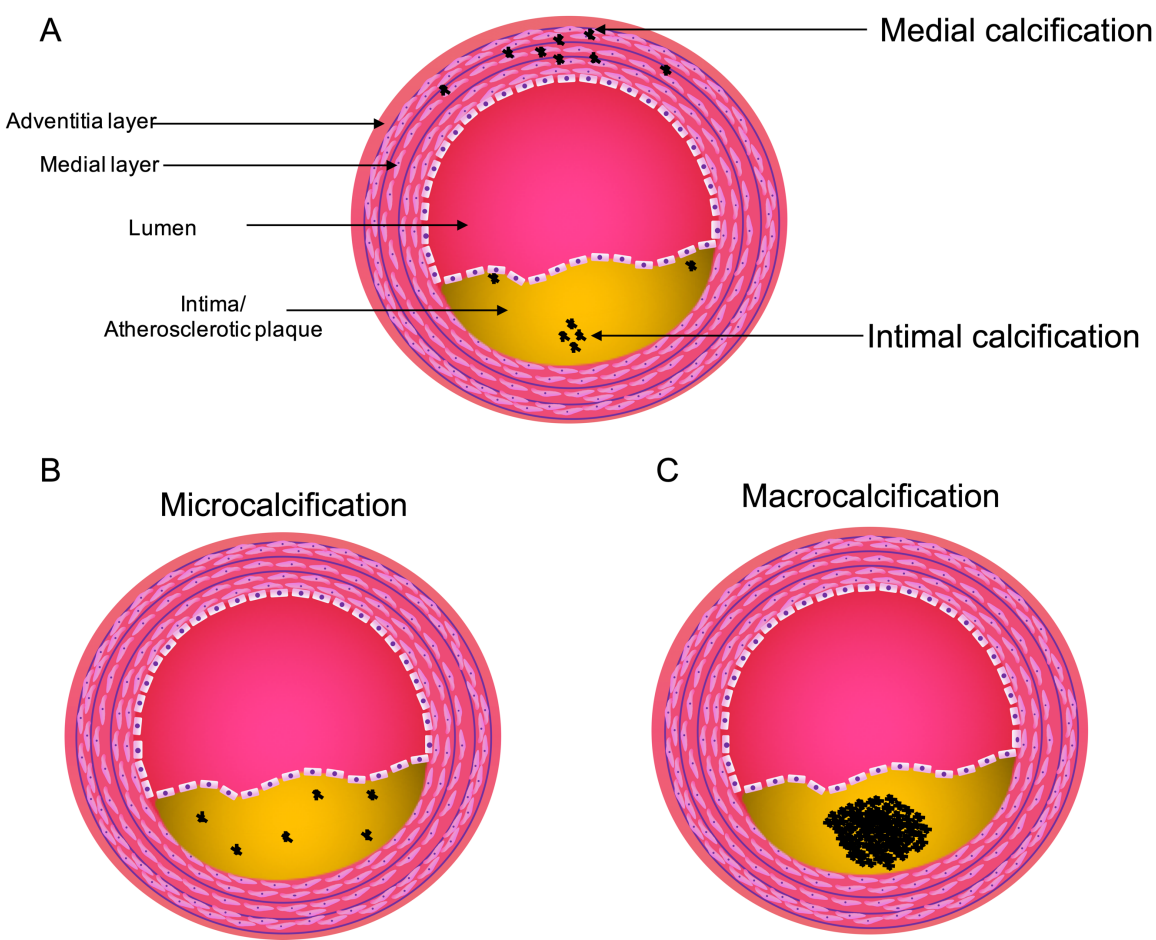

Figure 1. Crossover section of the lumen. Demonstration the vessel wall with calcification in the medial and intimal layer (A). Calcification can be distinguished based on size for micro (B) and macro calcification (C).

\section{Micro- and macrocalcification}

Calcium deposits $<50 \mu \mathrm{m}$ are considered as microcalcification. Microcalcification present in the atherosclerotic plaque increases plaque vulnerability (23). Microcalcification represents the early phase of vascular calcification, initiated by extracellular vesicles including exosomes and apoptotic bodies $(24,25)$. In the atherosclerotic plaque it has been shown that microcalcification and inflammation are closely related in vivo (26) and in vitro (27). Microcalcification can be visualized by invasive methods such as histopathology of vascular sections and intravascular ultrasound (IVUS). It has been associated with high risk and culprit atherosclerotic plaques (28), likely because it affects distribution of blood flow induced mechanical stress on the cap of the atherosclerotic plaque $(23,29)$. Moreover, microcalcification has been associated with active remodeling of atherosclerotic plaques thereby contributing to increasing plaque vulnerability (28). While intimal calcification has frequently been observed in advanced atherosclerotic plaque, it can also be detected in early stages of atherosclerosis (Table 1) (27). This raises the question whether microcalcification propels atherogenesis or that it is a consequence of atherosclerosis.

Non-invasive visualization of microcalcification has been challenging until recently. The resolution of computed tomography (CT) scanning is too low to detect 
microcalcifications. A recent breakthrough was achieved by employing PET imaging with the radiotracer ${ }^{18} \mathrm{~F}-\mathrm{NaF}$. This protocol is able to visualize active calcifications and identifies microcalcifications $(30,31)$. The incorporation of ${ }^{18} \mathrm{~F}$ into the calcium deposits is based on the exchange of fluoride ions with hydroxyl groups in hydroxyapatite. The uptake of ${ }^{18} \mathrm{~F}$ $\mathrm{NaF}$ is increased when microcalcifications are present because of their high surface to volume ratios as compared to macrocalcifications.

When calcification progresses and calcium deposits are $\geq 50 \mu \mathrm{m}$ it is considered macrocalcification. Microcalcification as detected by $\mathrm{NaF}^{18}$ has been shown to progress into macrocalcification detected by CT scanning $(32,33)$. Currently, macrocalcification is debated on its role in plaque stability (34). Prospective clinical data from the Multi-Ethnic Study of Atherosclerosis Trial (MESA) demonstrated an inverse correlation between arterial calcification per arterial volume whereas increased calcium density increased CVD risk (35). However, plaques with extensive calcification contribute to only a small percentage of total atherosclerotic plaque ruptures in acute coronary syndromes and sudden cardiac death (1), suggesting that calcium CT-score is merely a biomarker for plaque burden (36). Macrocalcification has been put forward to stabilize atherosclerotic plaques $(37,38)$. This is supported by data from patients treated with statins which increased coronary artery calcification progression while reducing plaque volume (39). Moreover, increased coronary artery calcification progression upon statin treatment is postulated to improve plaque repair rather than plaque volume (40), raising a possible hypothesis that statin treatment accelerates the conversion of microcalcification into macrocalcification (34). The mechanism behind statin increased plaque stability remains unknown. While micro- and macrocalcification are two different entities, both can be found in patients in close proximity (37).

Table 2. Characteristics of micro- and macrocalcification (14).

Microcalcification

\begin{tabular}{|c|c|}
\hline Small spots of calcification & Sheet like calcification \\
\hline Increased plaque vulnerability & Associated with plaque stability \\
\hline $\begin{array}{c}\text { Increased mechanical stress at the surface of } \\
\text { the atherosclerotic cap }\end{array}$ & \\
\hline Elevates inflammation & \\
\hline Detected by radiotracer $\mathrm{F}^{18}$ & Detected by CT \\
\hline
\end{tabular}

\section{Loss of calcification inhibitors}

Currently, there is no treatment for blocking, stopping or reducing vascular calcification in spite of the generally accepted notion that vascular calcification is a highly regulated process of which some molecular players and mechanisms have been elucidated (8). Fetuin-A and MGP have been identified as inhibitors of vascular calcification. MGP, a VKDP produced by VSMC in the vasculature, is a pivotal inhibitor of vascular calcification as revealed from MGP knockout (KO) mice which all died within 6-8 weeks after birth due to calcified and ruptured vessels (13). The lethal MGP KO phenotype could be restored by 
rescuing MGP expression in VSMC but not by increasing MGP in the circulation (41). VKA treatment, which impairs carboxylation of MGP, caused vascular calcification similar to the calcification observed in MGP KO mice (42). MGP inhibits calcification via multiple mechanisms. MGP can directly inhibit calcification via binding to growing hydroxy apatite crystals and thereby blocking nucleation sides. Indirectly, MGP blocks VSMC phenotype switching as $\mathrm{MGP}^{-/-}$mice demonstrate reduced contractile and increased osteochondrogenic markers in VSMC (8). However, MGP is not the only VKDP in vascular tissue that is affected by VKA. Gas6 and Upper zone of growth plate Cartilage Matrix Associated protein (UCMA; also known as Gla Rich Protein (GRP)) are two VKDPs which are inhibited by VKA treatment. Gas6 protects from VSMC apoptosis (43), thereby indirectly protecting from apoptosis since apoptosis promotes vascular calcification (44). UCMA blocks calcification via inhibiting VSMC phenotype switching towards osteochondrogenic differentiation (45).Vitamin $\mathrm{K}$ supplementation has been shown to increase carboxylation of VKDP such as MGP, suggesting that vitamin $\mathrm{K}$ supplementation has the potential for treatment of vascular calcification.

\section{Outline of thesis}

The research presented in this thesis aims to gain further insight into how oral anticoagulant treatment and vitamin $\mathrm{K}$ supplementation affects vascular remodeling. In more detail, I investigated the role of VSMC in vascular calcification and atherogenesis.

Chapter 2 focuses on the history and different aspects of oral anticoagulant treatments. This chapter compares oral anticoagulant treatment with VKA and NOAC. Moreover, direct and indirect effects of oral anticoagulant treatment and coagulation factors on arterial blood vessels are discussed. Chapter 3 investigates the role of vascular macrocalcification on the coagulation system. In a clinical cohort of patients on oral anticoagulation and in mice treated with oral anticoagulation I showed that vascular calcification is associated with a prethrombotic state. Additionally, I identified a potential mechanism by which VSMCs affect vascular remodeling thereby influencing thrombogenicity. Chapter $\mathbf{4}$ focuses on effects of VKA and vitamin $\mathrm{K}$ treatment on atherosclerosis development. Using a pre-clinical animal model, I studied the role of VKA and VKA replaced by vitamin K supplementation on atherosclerosis and vascular calcification. I present mechanistic insights into vitamin $\mathrm{K}$ dependent effects of VSMC on vascular remodeling. Chapter 5 elaborates on comparing VKA and NOAC treatment on atherosclerosis development. I emphasize the effect of VKA and NOAC on the development of intimal calcification. Using an immunohistochemical approach I demonstrate that long-term anticoagulation treatment has an aggravating or beneficial effect on atherogenesis for VKA and NOAC, respectively. In chapter 6 we investigate the role of Nox 5 as key factor in driving VSMC phenotype switching. Here, we show that Nox5 is a novel modulator of synthetic VSMCs and that synthetic VSMCs cause calcification via oxidative stress driven extracellular vesicle release. Chapter 7 summarizes key findings of my thesis and discusses them in relation with published literature. 


\section{Reference}

1. Insull W. The pathology of atherosclerosis: plaque development and plaque responses to medical treatment. Am J Med. 2009 Jan;122(1 Suppl):S3-S14.

2. Stary HC, Chandler AB, Dinsmore RE, Fuster V, Glagov S, Insull W, et al. A definition of advanced types of atherosclerotic lesions and a histological classification of atherosclerosis. A report from the Committee on Vascular Lesions of the Council on Arteriosclerosis, American Heart Association. Circulation. 1995 Sep 1;92(5):1355-74.

3. Stary HC. Natural history and histological classification of atherosclerotic lesions: an update. Arterioscler Thromb Vasc Biol. 2000 May;20(5):1177-8.

4. Previtali E, Bucciarelli P, Passamonti SM, Martinelli I. Risk factors for venous and arterial thrombosis. Blood Transfus. 2011 Apr;9(2):120-38.

5. Bentzon JF, Otsuka F, Virmani R, Falk E. Mechanisms of plaque formation and rupture. Circ Res. 2014 Jun 6;114(12):1852-66.

6. Badimon L, Vilahur G. Thrombosis formation on atherosclerotic lesions and plaque rupture. J Intern Med. 2014 Aug 22;276(6):618-32.

7. Willems BAG, Vermeer C, Reutelingsperger CPM, Schurgers LJ. The realm of vitamin K dependent proteins: Shifting from coagulation toward calcification. Mol Nutr Food Res. 2014 Aug;58(8):1620-35.

8. van Gorp RH, Schurgers LJ. New Insights into the Pros and Cons of the Clinical Use of Vitamin K Antagonists (VKAs) Versus Direct Oral Anticoagulants (DOACs). Nutrients. 2015;7(11):9538-57.

9. Schurgers LJ, Spronk HMH. Differential cellular effects of old and new oral anticoagulants: consequences to the genesis and progression of atherosclerosis. Thromb Haemost. 2014 Nov 3;112(5):909-17.

10. Patterson C, Stouffer GA, Madamanchi N, Runge MS. New Tricks for Old Dogs : Nonthrombotic Effects of Thrombin in Vessel Wall Biology. Circ Res. 2001 May 25;88(10):987-97.

11. Spronk HMH, de Jong AM, Crijns HJ, Schotten U, Van Gelder IC, Cate ten H. Pleiotropic effects of factor Xa and thrombin: what to expect from novel anticoagulants. Cardiovasc Res. 2014 Mar 1;101(3):344-51.

12. Borissoff JI, Otten JJT, Heeneman S, Leenders P, van Oerle R, Soehnlein O, et al. Genetic and pharmacological modifications of thrombin formation in apolipoprotein e-deficient mice determine atherosclerosis severity and atherothrombosis onset in a neutrophil-dependent manner. PLoS ONE. 2013;8(2):e55784.

13. Luo G, Ducy P, McKee MD, Pinero GJ, Loyer E, Behringer RR, et al. Spontaneous calcification of arteries and cartilage in mice lacking matrix GLA protein. Nature. 1997 Mar 6;386(6620):78-81.

14. Schurgers LJ, Joosen IA, Laufer EM, Chatrou MLL, Herfs M, Winkens MHM, et al. Vitamin Kantagonists accelerate atherosclerotic calcification and induce a vulnerable plaque phenotype. PLoS ONE. 2012;7(8):e43229.

15. Weijs B, Blaauw Y, Rennenberg RJMW, Schurgers LJ, Timmermans CCMM, Pison L, et al. Patients using vitamin $\mathrm{K}$ antagonists show increased levels of coronary calcification: an observational study in low-risk atrial fibrillation patients. Eur Heart J. 2011 Oct;32(20):2555-62.

16. Rennenberg RJMW, Kessels AGH, Schurgers LJ, van Engelshoven JMA, de Leeuw PW, Kroon AA. Vascular calcifications as a marker of increased cardiovascular risk: a meta-analysis. Vasc Health Risk Manag. 2009;5(1):185-97.

17. Hoffmann U, Massaro JM, D'Agostino RB, Kathiresan S, Fox CS, O'Donnell CJ. Cardiovascular Event Prediction and Risk Reclassification by Coronary, Aortic, and Valvular Calcification in the Framingham Heart Study. J Am Heart Assoc. 2016 Feb 22;5(2).

18. Vliegenthart R, Oudkerk M, Hofman A, Oei H-HS, van Dijck W, van Rooij FJA, et al. Coronary calcification improves cardiovascular risk prediction in the elderly. Circulation. 2005 Jul 26;112(4):5727.

19. Martin SS, Blaha MJ, Blankstein R, Agatston A, Rivera JJ, Virani SS, et al. Dyslipidemia, coronary artery calcium, and incident atherosclerotic cardiovascular disease: implications for statin therapy from the multi-ethnic study of atherosclerosis. Circulation. 2014 Jan 7;129(1):77-86.

20. Raggi P, Callister TQ, Shaw LJ. Progression of coronary artery calcium and risk of first myocardial infarction in patients receiving cholesterol-lowering therapy. Arterioscler Thromb Vasc Biol. American Heart Association, Inc; $2004 \mathrm{Jul} ; 24(7): 1272-7$.

21. Amann K. Media calcification and intima calcification are distinct entities in chronic kidney disease. Clin J Am Soc Nephrol. American Society of Nephrology; 2008 Nov;3(6):1599-605.

22. Rogers M, Goettsch C, Aikawa E. Medial and intimal calcification in chronic kidney disease: stressing the contributions. J Am Heart Assoc. 2013 Sep 23;2(5):e000481.

23. Vengrenyuk Y, Carlier S, Xanthos S, Cardoso L, Ganatos P, Virmani R, et al. A hypothesis for vulnerable plaque rupture due to stress-induced debonding around cellular microcalcifications in thin fibrous caps. Proc Natl Acad Sci USA. 2006 Oct 3;103(40):14678-83. 
24. New SEP, Goettsch C, Aikawa M, Marchini JF, Shibasaki M, Yabusaki K, et al. Macrophage-derived matrix vesicles: an alternative novel mechanism for microcalcification in atherosclerotic plaques. Circ Res. 2013 Jun 21;113(1):72-7.

25. Kapustin AN, Shanahan CM. Emerging roles for vascular smooth muscle cell exosomes in calcification and coagulation. J Physiol (Lond). 2016 Jun 1;594(11):2905-14.

26. Aikawa E, Nahrendorf M, Figueiredo J-L, Swirski FK, Shtatland T, Kohler RH, et al. Osteogenesis associates with inflammation in early-stage atherosclerosis evaluated by molecular imaging in vivo. Circulation. 2007 Dec 11;116(24):2841-50.

27. Chatrou MLL, Cleutjens JP, van der Vusse GJ, Roijers RB, Mutsaers PHA, Schurgers LJ. Intra-Section Analysis of Human Coronary Arteries Reveals a Potential Role for Micro-Calcifications in Macrophage Recruitment in the Early Stage of Atherosclerosis. PLoS ONE. 2015;10(11):e0142335.

28. Ehara S, Kobayashi Y, Yoshiyama M, Shimada K, Shimada Y, Fukuda D, et al. Spotty calcification typifies the culprit plaque in patients with acute myocardial infarction: an intravascular ultrasound study. Circulation. 2004 Nov 30;110(22):3424-9.

29. Kelly-Arnold A, Maldonado N, Laudier D, Aikawa E, Cardoso L, Weinbaum S. Revised microcalcification hypothesis for fibrous cap rupture in human coronary arteries. Proc Natl Acad Sci USA. National Academy of Sciences; 2013 Jun 25;110(26):10741-6.

30. Bucerius J, Dijkgraaf I, Mottaghy FM, Schurgers LJ. Target identification for the diagnosis and intervention of vulnerable atherosclerotic plaques beyond $18 \mathrm{~F}$-fluorodeoxyglucose positron emission tomography imaging: promising tracers on the horizon. Eur J Nucl Med Mol Imaging. Springer Berlin Heidelberg; 2019 Jan;46(1):251-65.

31. Irkle A, Vesey AT, Lewis DY, Skepper JN, Bird JLE, Dweck MR, et al. Identifying active vascular microcalcification by (18)F-sodium fluoride positron emission tomography. Nat Comms. 2015;6:7495.

32. Dweck MR, Jenkins WSA, Vesey AT, Pringle MAH, Chin CWL, Malley TS, et al. 18F-sodium fluoride uptake is a marker of active calcification and disease progression in patients with aortic stenosis. Circ Cardiovasc Imaging. 2014 Mar;7(2):371-8.

33. Pawade TA, Newby DE, Dweck MR. Calcification in Aortic Stenosis. J Am Coll Cardiol. 2015 Aug;66(5):561-77.

34. Reutelingsperger C, Schurgers L. Coronary Artery Calcification: A Janus-Faced Biomarker? JACC Cardiovasc Imaging. 2018 Sep;11(9):1324-6.

35. Criqui MH, Denenberg JO, Ix JH, McClelland RL, Wassel CL, Rifkin DE, et al. Calcium density of coronary artery plaque and risk of incident cardiovascular events. JAMA. American Medical Association; 2014 Jan 15;311(3):271-8.

36. Dweck MR, Aikawa E, Newby DE, Tarkin JM, Rudd JHF, Narula J, et al. Noninvasive Molecular Imaging of Disease Activity in Atherosclerosis. Circ Res. 2016 Jul 8;119(2):330-40.

37. Hutcheson JD, Goettsch C, Bertazzo S, Maldonado N, Ruiz JL, Goh W, et al. Genesis and growth of extracellular-vesicle-derived microcalcification in atherosclerotic plaques. Nat Mater. 2016 Jan 11.

38. Libby P. How does lipid lowering prevent coronary events? New insights from human imaging trials. Eur Heart J. 2015 Feb 21;36(8):472-4.

39. Puri R, Nicholls SJ, Shao M, Kataoka Y, Uno K, Kapadia SR, et al. Impact of statins on serial coronary calcification during atheroma progression and regression. J Am Coll Cardiol. 2015 Apr 7;65(13):127382.

40. Henein M, Granåsen G, Wiklund U, Schmermund A, Guerci A, Erbel R, et al. High dose and long-term statin therapy accelerate coronary artery calcification. International Journal of Cardiology. Elsevier Ireland Ltd; 2015 Apr 1;184(C):581-6.

41. Murshed M, Schinke T, McKee MD, Karsenty G. Extracellular matrix mineralization is regulated locally; different roles of two gla-containing proteins. J Cell Biol. 2004 Jun 7;165(5):625-30.

42. Price PA, Faus SA, Williamson MK. Warfarin causes rapid calcification of the elastic lamellae in rat arteries and heart valves. Arterioscler Thromb Vasc Biol. 1998 Sep;18(9):1400-7.

43. Melaragno MG, Cavet ME, Yan C, Tai L-K, Jin Z-G, Haendeler J, et al. Gas6 inhibits apoptosis in vascular smooth muscle: role of Axl kinase and Akt. J Mol Cell Cardiol. 2004 Oct;37(4):881-7.

44. Proudfoot D, Skepper JN, Hegyi L, Bennett MR, Shanahan CM, Weissberg PL. Apoptosis regulates human vascular calcification in vitro: evidence for initiation of vascular calcification by apoptotic bodies. Circ Res. Lippincott Williams \& Wilkins; 2000 Nov 24;87(11):1055-62.

45. Willems BA, Furmanik M, Caron MMJ, Chatrou MLL, Kusters DHM, Welting TJM, et al. Ucma/GRP inhibits phosphate-induced vascular smooth muscle cell calcification via SMAD-dependent BMP signalling. Sci Rep. Nature Publishing Group; 2018 Mar 21;8(1):4961. 
GENERAL INTRODUCTION 


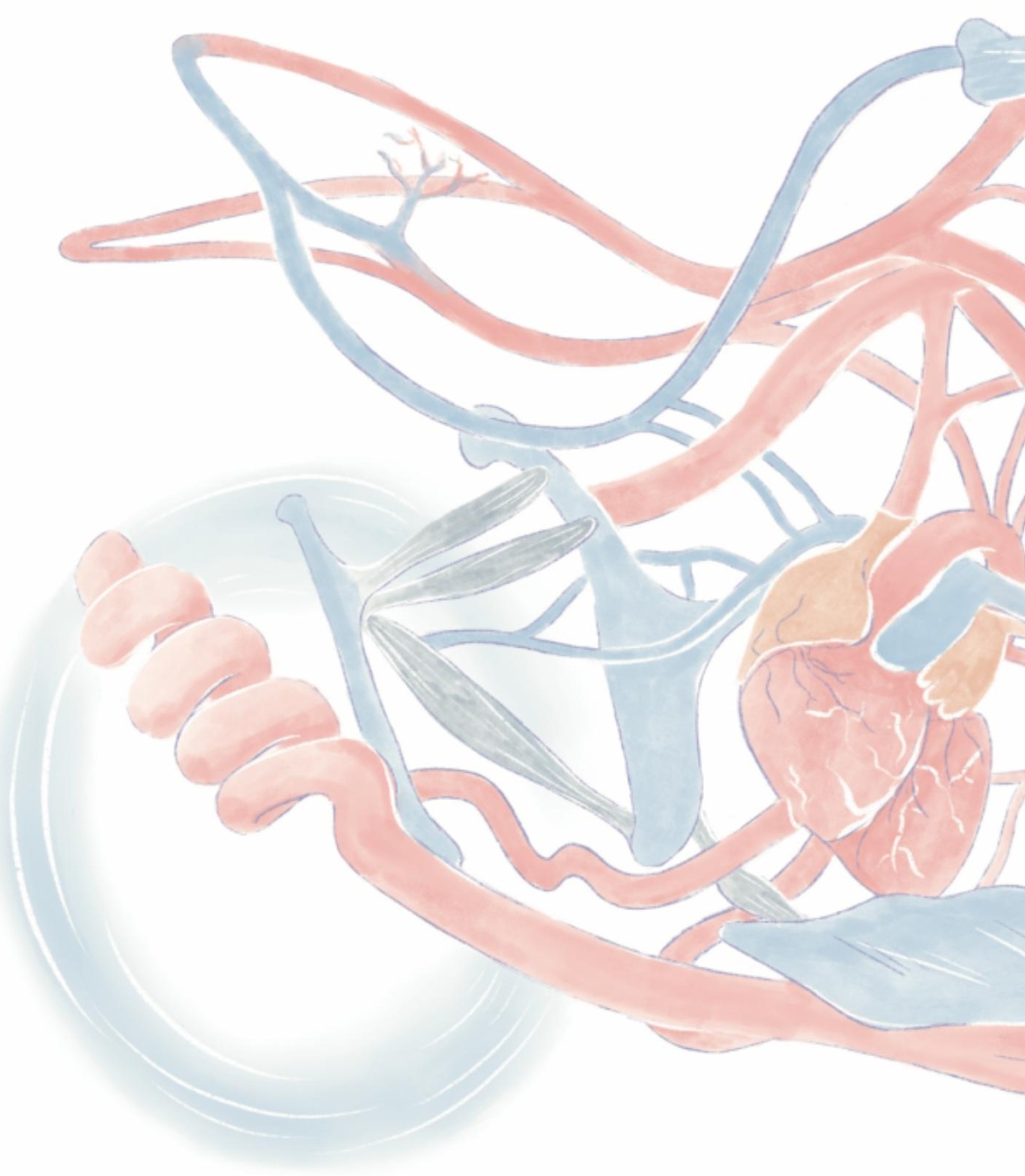




\section{Chapter 2}

New Insights into the Pros and Cons of the Clinical Use of Vitamin K Antagonists (VKAs) Versus Direct Oral Anticoagulants (DOACs)

Rick H. van Gorp and Leon J. Schurgers

Nutrients, 2015 vol.7 (11) pp. 9538-9557 


\begin{abstract}
Vitamin K-antagonists (VKA) are the most widely used anticoagulant drugs to treat patients at risk of arterial and venous thrombosis for the past 50 years. Due to unfavorable pharmacokinetics VKA have a small therapeutic window, require frequent monitoring, and are susceptible to drug and nutritional interactions. Additionally, the effect of VKA is not limited to coagulation, but affects all vitamin K-dependent proteins. As a consequence, VKA have detrimental side effects by enhancing medial and intimal calcification. These limitations stimulated the development of alternative anticoagulant drugs, resulting in direct nonvitamin $\mathrm{K}$ oral anticoagulant (DOAC or NOAC) drugs, which specifically target coagulation factor Xa and thrombin. NOACs also display non-hemostatic vascular effects via proteaseactivated receptors (PARs). As atherosclerosis is characterized by a hypercoagulable state indicating the involvement of activated coagulation factors in the genesis of atherosclerosis, anticoagulation could have beneficial effects on atherosclerosis. Additionally, accumulating evidence demonstrates vascular benefit from high vitamin $\mathrm{K}$ intake. This review gives an update on oral anticoagulant treatment on the vasculature with a special focus on calcification and vitamin $\mathrm{K}$ interaction.
\end{abstract}




\section{Arterial and Venous Thrombosis}

In 1856 Rudolf Virchow, often regarded as the founder of modern pathology, delineated three major components that were responsible for the formation of emboli in the venous circulation. These three elements, now known as Virchow's triad, can be briefly summarized as: (1) changes in the composition of blood; (2) alterations in the vessel wall; and (3) disruption of the blood flow. Coagulation is a protective response after vascular injury to prevent bleeding [1] and can be initiated via either the so-called intrinsic or extrinsic pathways, which although simplistic, are still useful schematic models of the coagulation process (Figure 1a). Both pathways are characterized by a series of enzymatic events whereby the activation of members of a hierarchical chain of coagulation enzymes (called coagulation factors) are successively activated by the preceding factor in the chain. Although the initiation steps are different, both pathways converge and lead to activation of prothrombin (FII) to produce thrombin (FIIa). An important feature of this coagulation cascade is that it functions as a biochemical amplifier [2] in which the final product, thrombin catalyses the production of fibrin which forms a meshwork clot [3]. The coagulation events leading to the formation of a blood clot (thrombus) that adheres to the wall of a blood vessel and obstructs the flow of blood is termed thrombosis. Thrombosis can take place in both arteries and veins. Atherothrombosis is the term describing the occlusion of a blood vessel by a ruptured atherosclerotic plaque $[4,5]$. Arterial thrombosis can lead to stroke and myocardial infarction. In contrast to arterial thrombosis, venous thrombosis is associated with dysregulation of coagulation proteins and manifests in deep-vein thrombosis and pulmonary embolism [6]. Obesity and diabetes mellitus are risk factors for both arterial and venous thrombosis whereas other risk factors such as smoking, hypertension and hyperlipidemia increase only the risk for arterial thrombosis [6]. Oral anticoagulant drugs are prescribed to patients to reduce the risk and incidence of both arterial and venous thrombosis, although mainly for the latter.

\section{The Discovery of Oral Anticoagulant Drugs}

The story of the discovery of vitamin K antagonists (VKA) began in the 1920s as a result of an often fatal bleeding disorder in cattle that manifested after the animals had been fed on the hay derived from sweet clover [7]. For this reason the haemorrhagic disease became known as "sweet clover disease". A crucial observation was the animals that bled had been fed on sweet clover hay that had become mouldy; those animals fed mould-free hay did not present with bleeding [7]. During the subsequent classical studies by Karl Link's group in Wisconsin it was first shown that bleeding was associated with a low plasma activity of prothrombin as measured by early coagulation assays that were the precursors of the modern day prothrombin time. Link's group then undertook the task of isolating the haemorrhagic component in spoiled sweet clover that was responsible for the prolonged 


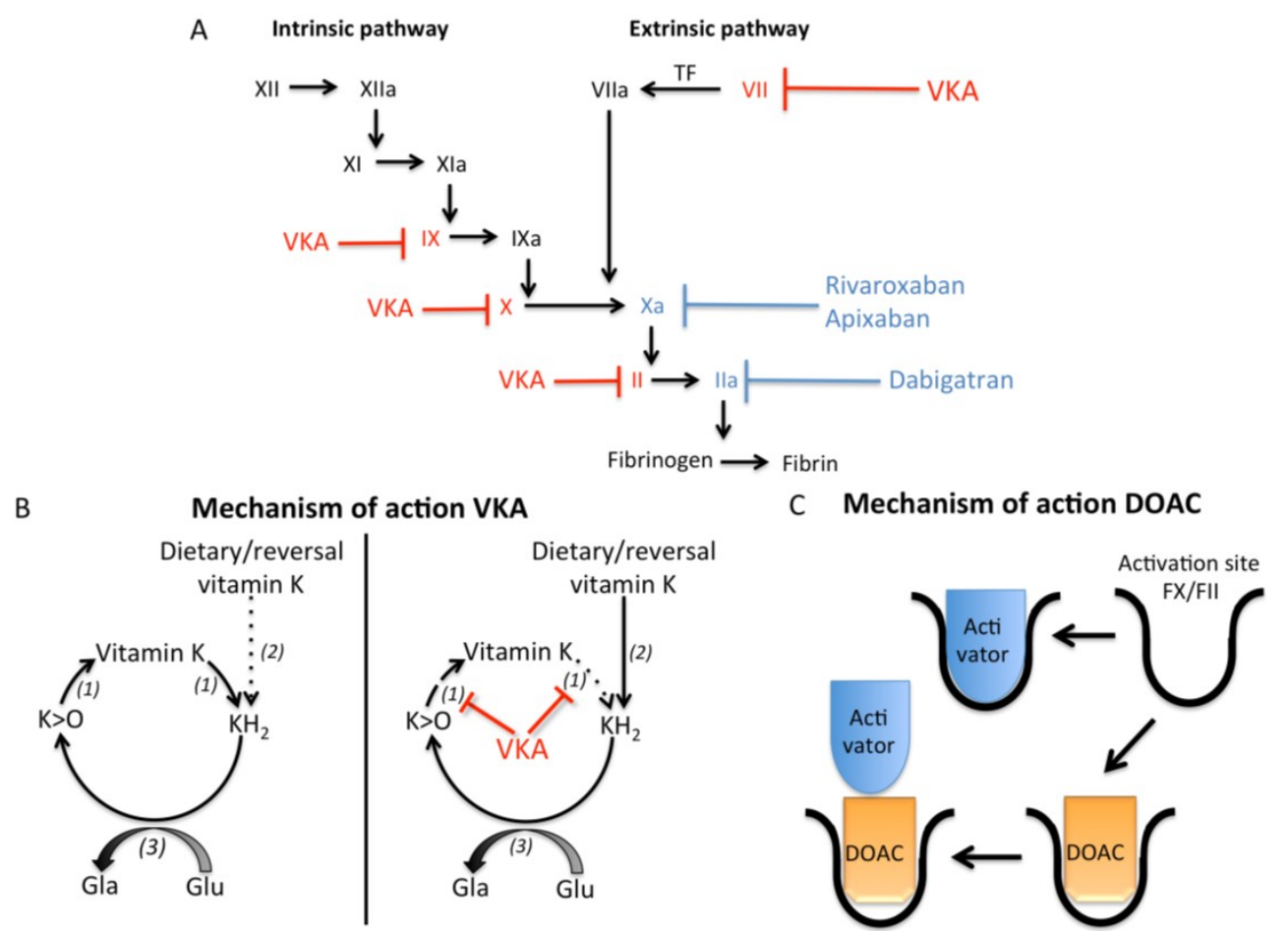

Figure 1. Effects of vitamin $\mathrm{K}$ antagonists and direct oral anticoagulants on coagulation. (A) The coagulation cascade can be activated by both the intrinsic and extrinsic pathway, which finally results in activation of thrombin and subsequently fibrin formation. Vitamin K antagonists (VKA) induce anticoagulation via inhibiting activation of the coagulation factors depicted in red (factors X, IX, VII, and II). Direct non-vitamin K oral anticoagulants (DOACs or NOAC) induce anticoagulation via blocking the activity of coagulation factors Xa (rivaroxaban and apixaban) and IIa (dabigatran) depicted in blue; (B) Vitamin K cycle is required to carboxylate, and thus activate, vitamin $\mathrm{K}$ dependent proteins. Vitamin $\mathrm{K}$ is converted to vitamin hydroquinone $\left(\mathrm{KH}_{2}\right)$, which is oxidized by $\gamma$ glutamylcarboxylase (3) to convert glutamate (Glu) residues in $\gamma$-carboxyglutamate (Gla) residues. This reaction results in vitamin $\mathrm{K}$ epoxide $(\mathrm{K}>\mathrm{O})$, which is recycled to vitamin $\mathrm{K}$ through vitamin $\mathrm{K}$ epoxide reductase (1). VKA disrupts the vitamin $\mathrm{K}$ cycle by inhibiting vitamin $\mathrm{K}$ epoxide reductase (VKOR) leading to depletion of vitamin $\mathrm{K}$ and uncarboxylated vitamin $\mathrm{K}$ dependent proteins. In the liver, the inhibition of warfarin can be circumvented via $\mathrm{NAD}(\mathrm{P}) \mathrm{H}$ quinone reductase (2), which can convert vitamin $\mathrm{K}$ into $\mathrm{KH}_{2}$ even in the presence of VKA. In extrahepatic tissues $\mathrm{NAD}(\mathrm{P}) \mathrm{H}$ quinone reductase activity is $c a .100$ fold less, resulting in inactive vitamin $\mathrm{K}$ dependent proteins in the presence of VKA; (C) NOACs induce anticoagulation via inhibiting the activity of FXa and FIIa via binding to the activation site.

prothrombin time. This proved a long and arduous process but finally resulted in the isolation and identification of the haemorrhagic agent that we now know as the compound dicoumarol and the first VKA [8]. Dicoumarol is a 4-hydroxycoumarin derivative and originated from microbial action on the compound coumarin which is rich in sweet clover.

After animal trials, dicoumarol was rapidly introduced into the clinic with considerable success. At that time, dicoumarol had also been tested in field trials as a potential rodenticide but it was concluded that the anticoagulant activity of dicoumarol in the rat was not high enough to make it practical for rodent control [8]. Therefore, Link's group synthesized a 
large number of different 4-hydroxycoumarin compounds to try and find a derivative with increased anticoagulant activity over dicoumarol. It turned out that a 4-hydroxycoumarin anticoagulant number 42 (out of 150 synthetic variants) was more potent than dicoumarol and had superior pharmacokinetics [8]. This coumarin derivative is today known as warfarin, the word originating from the combination of the Wisconsin Alumni Research Foundation (WARF) and "arin" from coum"arin". Warfarin revolutionized rodent control but clinicians were reluctant to use a rat poison in humans until an unforeseen event changed their minds. In 1951 an army conscript tried to commit suicide with warfarin. According to Link the slow onset time of warfarin gave him "too much time for thinking" enabling him to reconsider and admit himself to hospital where after receiving vitamin $\mathrm{K}$ supplementation he completely recovered. Clinical trials with warfarin in the 1950s confirmed its superiority over dicoumarol, being better absorbed and some 5-10 times more potent, although no head to head comparison was performed. Moreover, it became apparent that an overdose could easily be corrected by vitamin $\mathrm{K}$ supplementation $[8,9]$. Today, warfarin is the most prescribed oral anticoagulant world-wide [10].

\subsection{Vitamin $K$ and Vitamin $K$ Antagonists}

Vitamin $\mathrm{K}$ is a fat-soluble vitamin that exists in different forms (Table 1). All forms of vitamin $\mathrm{K}$ contain a 2-methyl-1,4-naphthoquinone ring structure, also known as menadione (vitamin $\mathrm{K}_{3}$ ). Phylloquinone (vitamins $\mathrm{K}_{1}$ ) and menaquinones (vitamin $\mathrm{K}_{2}$ ) are classified according to the length and degree of saturation of the aliphatic side chain. Phylloquinone contains a phytyl side chain, and its main dietary sources are green leafy vegetables and certain vegetable oils. Menaquinones have an unsaturated aliphatic side chain comprising a varying number of prenyl units, abbreviated as MK-n (menaquinone with $\mathrm{n}$ representing the number of prenyl units). All menaquinones, except MK-4, are produced by bacteria and can be found in fermented foods such as cheese. Additionally, some bacteria in our intestines produce long-chain menaquinones (mainly MK-10 and MK-11) and although they make up the majority of human hepatic reserves their bioavailability for the synthesis of coagulation Gla proteins in the liver is debatable [11]. Both phylloquinone and menaquinones can participate in the $\gamma$-glutamylcarboxylation of both hepatic and extra-hepatic vitamin $\mathrm{K}$ dependent proteins, although the longer residence times and better absorption of long chain menaquinones such as MK-7 and MK-9 in the circulation [12, 13] makes them more effective for carboxylating both hepatic and extrahepatic vitamin K-dependent proteins [14]. Interestingly, menadione has been shown to act as an endogenous metabolite formed during the in vivo conversion of phylloquinone to MK-4 [15]. Furthermore, whereas phylloquinone and menaquinones can reverse VKA induced anticoagulation, menadione per se has no cofactor activity for $\gamma$-carboxylation and thus cannot reverse VKA-induced anticoagulation.

The molecular function of vitamin $\mathrm{K}$ is to serve as an essential cofactor to drive the $\gamma$ glutamyl carboxylation reaction (Figure 1b). In this vitamin K-dependent reaction, specific 
protein bound glutamate residues are modified to $\gamma$-glutamate residues, hence the name of the $\gamma$-carboxylated protein products as vitamin K-dependent proteins (VKDP).

To achieve this protein modification vitamin $\mathrm{K}$ is first reduced to the active cofactor vitamin hydroquinone $\left(\mathrm{KH}_{2}\right)$ via quinone reductases. Next, the enzyme $\gamma$-glutamyl carboxylase (GGCX) oxidizes vitamin $\mathrm{KH}_{2}$ with vitamin $\mathrm{K}$ epoxide $(\mathrm{K}>\mathrm{O})$ as the product. This oxidation reaction is intimately linked and essential to the $\gamma$-carboxylation modification of VKDP [16]. The metabolite $\mathrm{K}>\mathrm{O}$ can be recycled by the microsomal enzyme vitamin $\mathrm{K}$ epoxide reductase (VKOR), first to vitamin $\mathrm{K}$ and then to $\mathrm{KH}_{2}$. This cyclic pathway is called the "vitamin K-epoxide cycle" or simply the "vitamin K cycle" [17, 18]. By this salvage mechanism, one molecule of vitamin $\mathrm{K}$ is able to carboxylate some 500 glutamate residues [17].

Table 1. Structural forms of vitamin $\mathrm{K}$.

\begin{tabular}{|c|c|c|c|}
\hline Drug & \multicolumn{2}{|c|}{ Characterization } & Dietary Sources \\
\hline Phylloquinone (vitamin $\mathrm{K}_{1}$ ) & \multicolumn{2}{|c|}{ Phytyl side chain } & Leafy green vegetables \\
\hline \multirow{3}{*}{ Menaquinones (vitamin $\mathrm{K}_{2}$ ) } & \multirow{3}{*}{ Isoprenoid side chain } & MK-4 & Meat, eggs \\
\hline & & MK-7 & Natto, Cheese \\
\hline & & MK-9 & Cheese, curd, sauerkraut \\
\hline Menadione (vitamin $\mathrm{K}_{3}$ ) & \multicolumn{2}{|c|}{ 2-methyl-1,4- naphthoquinone } & $\begin{array}{c}\text { Non-dietary metabolite. } \\
\text { Precursor of MK-4 }\end{array}$ \\
\hline
\end{tabular}

A deficiency of vitamin $\mathrm{K}$ can result from an insufficient dietary intake of vitamin $\mathrm{K}$ leading to depletion of local vitamin $\mathrm{K}$ tissue stores or via interference with the vitamin $\mathrm{K}$ cycle by VKA $[18,19]$. VKA exert their anticoagulant effect by inhibiting the VKOR enzyme resulting in reduced recycling of $\mathrm{K}>\mathrm{O}$, thereby limiting $\mathrm{KH}_{2}$ production. As the cellular concentrations of $\mathrm{KH}_{2}$ decline a stage is reached when the cofactor supply to the GGCX becomes insufficient to fully carboxylate the VKDP that are synthesized in a particular tissue. This in turn leads to the secretion of undercarboxylated species of VKDP in the circulation. The anticoagulant balance exerted by VKA can be said to be ultimately determined by the concentrations of $\gamma$-carboxylated coagulation proteins II, VII, IX and X that are secreted into the circulation. Changes in nutritional vitamin $\mathrm{K}$ intake interfere with VKA treatment by altering the size of the available pool of $\mathrm{KH}_{2}$ cofactor because dietary vitamin $\mathrm{K}$ in its quinone state can be converted to $\mathrm{KH}_{2}$ by a dehydrogenase enzyme not affected by VKA [18]. Remarkably, despite the long use of VKA the exact mechanism of inhibition of VKOR remains to be elucidated [18].

Owing to their unfavorable pharmacokinetics, VKA have a small therapeutic window, require frequent monitoring, and are susceptible to drug and nutritional interactions. A major disadvantage is that because of their indirect mechanism of action there is a lag phase of 23 days before a therapeutic anticoagulant effect is achieved. Therefore, considerable resources have been directed to the discovery of new anticoagulant agents that can directly target specific factors in the coagulation cascade. Several of these so-called direct non- 
vitamin $\mathrm{K}$ oral anticoagulants (DOACs or NOACs) have been approved for clinical use and can be subdivided into agents that either target coagulation factor IIa (FIIa, thrombin) or factor Xa (FXa).

\subsection{Direct Thrombin Inhibitors}

As with the discovery of VKA, the presence in nature of another anticoagulant (albeit in this case an anticoagulant without a lag phase) proved a catalyst to the future discovery of direct inhibitors of coagulation factors. Here it was the isolation of hirudin, a peptide present in the saliva of leeches as a direct inhibitor of thrombin, and long known to prevent coagulation [20, 21]. For a detailed review on hirudin, see [22]. Hirudin binds to both the active and substrate recognition sites of thrombin. In addition, it is slowly reversible and excreted predominantly by the kidneys. In the 1990s, recombinant hirudin was shown to prevent postoperative venous thromboembolism [23]. However, a major concern was the increased bleeding tendency with hirudin treatment $[21,24]$. As with heparin another major disadvantage of hirudin is that it could only be administered by subcutaneous injection. Despite the specific concerns with hirudin, the theory behind designing a direct thrombin inhibitor as a clinical anticoagulant gained support and in the early 2000s a compound called ximelagatran became the first synthetic direct inhibitor of thrombin to be trialed as an oral anticoagulant. However, although initial human trials were promising, later trials led to concerns of hepatotoxicity, which ultimately prevented ximelagatran from being used in the clinical setting $[25,26]$.

The second direct oral anticoagulant to undergo clinical trials was dabigatran etexilate, and this agent received approval for clinical use in 2008 (Table 2). Dabigatran etexilate is a prodrug that requires hydrolysis by carboxylesterases in the body to the active metabolite dabigatran [27]. Dabigatran binds with high specificity to the active site of thrombin thereby inhibiting both bound and free thrombin activity (Figure 1c). It takes two hours before dabigatran etexilate is metabolically active, which eliminates the need for parental anticoagulation. Dabigatran has a half-life of $12-17 \mathrm{~h}$ and is usually taken twice daily. Since $80 \%$ of dabigatran is excreted by the kidneys, patients with renal problems are not suited for this drug $[25,28,29]$.

\subsection{Factor Xa Inhibitors}

The idea of using FXa inhibitors as clinical anticoagulants also originates from naturally occurring inhibitors. The first FXa inhibitor to be studied was a compound called antistasin, originally isolated from the Mexican leech haementeria officinalis [33]. However, the concept of FXa inhibitors as anticoagulant drugs was not supported until a second FXa inhibitor called the tick anticoagulant peptide (TAP) had been isolated from the soft tick ornithodors moubata [34]. In vitro and in vivo studies demonstrated that FXa inhibitors block the activity of FXa generated via both intrinsic and extrinsic pathways and thus subsequently block the formation of thrombin $[35,36]$. 
In 2012 the FXa inhibitor called rivaroxaban was approved for clinical use (Table 2). Rivaroxaban acts via inhibition of the active site of FXa (Figure 1c) [35], and has predictable pharmacokinetic and dynamics [30]. Peak activity of rivaroxaban occurs $2-3 \mathrm{~h}$ after intake, with a half -life of 5-9 h [30]. The short half-life suggests that rivaroxaban needs to be taken twice daily, however guidelines for rivaroxaban usage recommend once daily. This recommendation comes from both clinical phase II and III trials, which provided evidence that once daily administration is most beneficial with respect to the balance between safety and efficacy [37]. In addition, the duration of rivaroxaban inhibiting FXa lasts $24 \mathrm{~h}$ thereby supporting the once daily policy. Rivaroxaban is mainly excreted by the kidneys $(66 \%$ with $36 \%$ as unchanged drug) with a smaller fraction excreted in the faeces $(28 \%$ with $7 \%$ unchanged) [31].

To date, the most recently approved NOAC is another FXa inhibitor called apixaban (Table 2). Like rivaroxaban, apixaban inhibits both bound and free FXa (Figure 1c).

Table 2. Characteristics of VKA and NOACs.

\begin{tabular}{|c|c|c|c|c|c|c|}
\hline & \multicolumn{3}{|c|}{ VKA [10] } & \multirow{2}{*}{$\begin{array}{c}\text { Dabigatra } \\
\text { n Etexilate } \\
{[24,27,28]}\end{array}$} & \multirow[b]{2}{*}{$\begin{array}{c}\text { Rivaroxab } \\
\text { an }[30,31]\end{array}$} & \multirow[b]{2}{*}{$\begin{array}{c}\text { Apixaban } \\
{[32]}\end{array}$} \\
\hline & Warfarin & $\begin{array}{c}\text { Acenocoumar } \\
\text { ol }\end{array}$ & $\begin{array}{c}\text { Phenprocoum } \\
\text { on }\end{array}$ & & & \\
\hline Target & $\begin{array}{l}\text { Vitamin K } \\
\text { epoxide } \\
\text { reductase }\end{array}$ & $\begin{array}{c}\text { Vitamin K } \\
\text { epoxide } \\
\text { reductase }\end{array}$ & $\begin{array}{c}\text { Vitamin K } \\
\text { epoxide } \\
\text { reductase }\end{array}$ & Thrombin & Factor Xa & Factor Xa \\
\hline Pro-drug & No & No & No & $\begin{array}{l}\text { Yes, active } \\
\text { metabolite } \\
\text { is } \\
\text { dabigatran }\end{array}$ & No & No \\
\hline $\begin{array}{c}\text { Half-life } \\
\text { (hours) }\end{array}$ & $20-60$ & $8-11$ & $120-144$ & $12-17$ & $5-9$ & $9-14$ \\
\hline $\begin{array}{l}\text { Onset time } \\
\text { peak effect } \\
\text { (hours) }\end{array}$ & $72-96$ & $36-48$ & $48-72$ & 2 & $2-3$ & 3 \\
\hline $\begin{array}{c}\text { Duration of } \\
\text { action }\end{array}$ & $2-5$ days & $<48 \mathrm{~h}$ & $7-14$ days & $24-36 \mathrm{~h}$ & $24 \mathrm{~h}$ & $24 \mathrm{~h}$ \\
\hline Metabolism & $\begin{array}{c}\text { Via } \\
\text { cytochrom } \\
\text { e P } 450\end{array}$ & $\begin{array}{c}\text { Via } \\
\text { cytochrome P } \\
450\end{array}$ & $\begin{array}{c}\text { Via } \\
\text { cytochrome P } \\
450\end{array}$ & $\begin{array}{c}\text { Via P- } \\
\text { Glucoprote } \\
\text { in } \\
\text { transporter }\end{array}$ & $\begin{array}{c}\text { Via } \\
\text { cytochrome } \\
\text { P450 } \\
(30 \%) \text {, and } \\
\text { P- } \\
\text { Glucoprotei } \\
n \\
\text { transporter }\end{array}$ & $\begin{array}{c}\text { Via } \\
\text { cytochrom } \\
\text { e P450 } \\
(15 \%) \text {, and } \\
\text { P- } \\
\text { Glucoprote } \\
\text { in } \\
\text { transporter }\end{array}$ \\
\hline Elimination & $\begin{array}{l}\text { Hepatical } \\
\text { metaboliz } \\
\text { ed }\end{array}$ & $\begin{array}{l}60 \% \text { Renal } \\
29 \% \text { Fecal }\end{array}$ & $\begin{array}{l}63 \% \text { Renal } \\
33 \% \text { Fecal }\end{array}$ & $\begin{array}{l}85 \% \text { Renal } \\
6 \% \text { Fecal }\end{array}$ & $\begin{array}{l}66 \% \text { Renal } \\
28 \% \text { Fecal }\end{array}$ & $25 \%$ Renal \\
\hline $\begin{array}{c}\text { Bioavailabili } \\
\text { ty }\end{array}$ & $\begin{array}{l}79 \%- \\
100 \%\end{array}$ & $60 \%$ & $>99 \%$ & $6.5 \%$ & $80 \%$ & $66 \%$ \\
\hline
\end{tabular}


Apixaban activity peaks $3 \mathrm{~h}$ after intake and has a half life of 9-14 h [32]. Bioavailability of apixaban is $66 \%$, and apixaban is partly (25\%) excreted by the kidneys.

\section{Clinical Trials with Oral Anticoagulation Drugs}

\subsection{Vitamin K Antagonists (VKA)}

The promise and later importance of VKA as oral anticoagulant drugs for clinical use became apparent in a randomized trial performed in the 1960s [38]. In this trial, patients with pulmonary embolism were divided in two groups receiving either the anticoagulant drug or placebo control. Of the group receiving anticoagulation therapy none of the patients died, whereas 5 patients in the placebo group died of pulmonary embolism [38].

Ever since the clinical introduction of VKA, their clinical efficacy and safety have been monitored through measuring the coagulation activity of the blood, mainly using the prothrombin time (PT) test or a close variant of this test [39]. A central ingredient of the PT test is a biological tissue reagent called thromboplastin. It quickly became apparent that innate variations in the source and batch of thromboplastin led to significant variabilities in PT results which were usually reported as a prothrombin time ratio (PTR) representing the patient's PT divided by normal PT [39]. In principle, when a high or low laboratory PTR is reported, the anticoagulant dosage is adjusted accordingly to reach the target coagulation ratio [39]. In the early 1960s it became apparent that some commercial thromboplastins were insufficiently responsive to the anticoagulant-induced effect leading to an underestimation of the dose of VKA required to achieve the target PTR. The subsequent overdosing with VKA led to an increase in bleeding complications and indicated the importance of using sensitive thromboplastin-based assays to prevent over or under dosing with VKA. Comparison of thromboplastin assays between North America and the UK revealed that increased sensitivity of assays reduced the incidence of hemorrhage [39, 40]. These results also addressed the need for increased standardization of PT assays and international guidelines for monitoring anticoagulation therapy. In 1983, the World Health Organization (WHO) adopted a universally standardized system of reporting patient PT data during VKA therapy called the international normalized ratio (INR) which is still used today [41].

\section{2. $N O A C s$}

With the approval of NOACs for clinical use, an alternative to VKA or heparin treatment became available. Recent clinical trials have investigated efficacy and safety profiles of NOACs in comparison to VKA treatment. The outcomes of these comparative trials are briefly described below.

\subsubsection{Dabigatran Etexilate}

The RE-LY and RE-COVER clinical trials demonstrated non-inferiority of dabigatran etexilate compared to warfarin in atrial fibrillation (AF) patients and patients with acute 
venous thromboembolism, respectively [25, 42]. Additionally, the RE-MODEL clinical trial demonstrated non-inferiority of dabigatran compared to enoxaparin treatment with respect to preventing venous thromboembolism after total knee replacement surgery [43]. Taken together these clinical trials demonstrated the non-inferiority of dabigatran compared to VKA treatment. Thus, dabigatran etexilate seems a suitable alternative for VKA for the treatment of patients with increased thrombosis risk.

\subsubsection{Rivaroxaban}

The clinical trials ROCKET AF, RECORD, EINSTEIN-DVT and EINSTEINExtension demonstrated the non-inferiority of rivaroxaban as compared to VKA and heparin treatment in patients with non-valvular atrial fibrillation, symptomatic venous thromboembolism, deep-vein thrombosis, and recurrent thrombosis in deep-vein thrombosis patients [44-46]. Moreover, rivaroxaban demonstrated no difference in risk for major bleedings in patients undergoing elective hip/knee replacement, though rivaroxaban was more effective in preventing symptomatic venous thromboembolism [47].

\subsubsection{Apixaban}

The ARISTOTLE and ADVANCE clinical trials showed non-inferiority of apixaban as compared to VKA and heparin in AF patients and for thromboprophylaxis in patients after hip replacement, respectively [48, 49]. Similar to dabigatran and rivaroxaban, apixaban showed no difference as compared to VKA treatment with respect to risk for major bleedings.

These clinical trials did not demonstrate superiority of NOACs over VKA, questioning whether NOACs should replace VKA as standard treatment. A meta-analysis comparing NOACs with VKA provided additional insight and showed the superiority over NOACs compared to VKA treatment with respect to major bleedings [50]. However, care is required interpreting these results and more research is needed.

\section{Advantages and Disadvantages of VKA and NOACs}

The use of VKA treatment over 60 years is one of the major advantages, since this revealed both short and long-term effects in humans. Disadvantages of VKA include the narrow therapeutic window and thus indirectly safety and efficacy. Therefore, VKA therapy requires regular monitoring by measuring the $\operatorname{INR}[21,51]$. Additionally, the pharmacokinetic and pharmacodynamics are unpredictable through drug interactions, cytochrome P450-dependent mechanisms, and the influence of dietary vitamin K intake [10, 52]. All the coumarin VKA listed in Table 2 (i.e., warfarin, acenocoumarol, and phenprocoumon) have a slow onset, taking $c a$. two to seven days to be effective in inducing anticoagulation $[10,21]$. Therefore, VKA therapy requires initial co-administration with other anticoagulants, such as heparin. Moreover, it has been shown recently that patients taking VKA treatment display hitherto unreported non-hemostasis side effects [53]. One of these side effects is VKA-induced vascular calcification. Vascular calcification is associated 
with an increased risk for cardiovascular disease [53-55]. In addition, VKA treatment is associated with arterial stiffness, which in turn is related to vascular calcification $[56,57]$.

NOACs were developed to circumvent the disadvantages of VKA therapy without negatively influencing safety profiles. Initially, the manufacturers of NOACs promoted the view that a major advantage of NOACs was that they did not require routine coagulation testing (as needed for VKA) or the measurement of circulating drug concentrations. However, data from the dabigatran RE-LY trial suggested that adjusting the dosage of dabigatran based on measurements of plasma dabigatran concentrations may reduce major bleeding events by as much as $30 \%-40 \%$ as compared to well-controlled warfarin [58]. Moreover, monitoring dabigatran plasma concentrations has the potential to improve safety and efficacy profiles as compared to fixed dosage $[58,59]$. Therapeutic drug monitoring of NOACs is supported by population studies which demonstrated a wide range of plasma dabigatran concentrations in different patient groups and at different time points although drug concentrations remained within the putative therapeutic range [60]. Variations in plasma concentration are ascribed to drug interactions, differences in absorption in the gastrointestinal tract, and clearance by the liver and kidneys [61]. These issues raise the question whether NOAC treatment should be regularly monitored.

A major issue of oral anticoagulation using VKA and NOACs is the need for reversal agents and coagulation assays to monitor the precise degree of anticoagulation. This is of importance in the event of bleeding and to allow surgical procedures or to counteract overdosing. The importance of reversal agents is illustrated by warfarin-related bleeding events, in which $50 \%$ of patients die within 90 days, mainly due to intracranial hemorrhage [62]. In the case of VKA, guidelines for the reversal of over-anticoagulation are well established with effective reversal agents (phylloquinone, and prothrombin complex concentrates) and sensitive coagulation assays (e.g., INR) to monitor haemostasis [62]. Vitamin $\mathrm{K}$ can counteract the effect of VKA via a NAD(P)H-dependent quinone reductase, the precise identity of which is uncertain. The enzyme that counteracts VKA is predominantly active in the liver and unlike VKOR is not inhibited by VKA thereby enabling the reduction of vitamin $\mathrm{K}$ into $\mathrm{KH}_{2}$ cofactors needed for $\gamma$-glutamyl carboxylation (Figure 1b) [63]. Reversal agents for NOACs were initially lacking, but are currently under development (idarucizumab for dabigatran, and andexanet and PER977 for FXa) [64]. Idarucizumab was developed to inactivate dabigatran, and works by binding to dabigatran with an affinity 350 times higher than thrombin. Recently, the clinical trial RE-VERSE AD investigated the capability and safety of idarucizumab as a reversal agent, and demonstrated a complete reversal of the anticoagulation effect of dabigatran within minutes. Of note, a major limitation in the RE-VERSE AD trial is the lack of a control group [65].

It should be noted that all NOACs are partially excreted by the kidneys $(80 \%, 65 \%$, and $25 \%$ for dabigatran, rivaroxaban and apixaban, respectively) $[66,67]$ and are therefore unsuitable for patients with severe renal deficiency. In contrast, VKA are predominantly 
metabolized through the liver and are thus the best option for anticoagulation with this patient population.

\section{Vitamin K Dependent Proteins and Atherosclerosis}

\subsection{Coagulation and Atherosclerosis}

Coagulation factors are effective activators of the vascular system independent of their effects on coagulation and exhibit pleiotropic effects on the vasculature that contribute to cardiovascular disease. More specifically, coagulation factors can affect the vessel wall through regulation of the proliferation, migration and differentiation of vascular smooth muscle cells (VSMCs) as well as by inducing oxidative stress, inflammation and apoptosis $[66,67]$, all processes that contribute to the development of atherosclerosis. In addition, micro plaque ruptures and subclinical thrombosis are pivotal to the progression and increased vulnerability of plaques, thereby increasing the risk for atherothrombosis [67].

\subsection{Thrombin and Atherosclerosis}

Thrombin is the central player in the coagulation cascade and influences nonhemostasis signaling via the protease-activated receptors (PAR-1, PAR-2, PAR-3, PAR-4) [66]. Thrombin activates these receptors via proteolytic cleavage of the N-terminal domain of PAR-1, PAR-3 and PAR-4 resulting in a tethered ligand activating the receptor [68, 69]. In the vasculature, endothelial cells express PAR-1, PAR-2 and PAR-4, whereas VSMCs express PAR-1 and PAR-2 [70]. The effects of thrombin on the different PARs are likely to induce different effects, of which PAR-1 related effects have been most studied $[66,71]$.

Human atherosclerotic plaques express elevated levels of PAR-1 [72], suggesting that PAR-1 plays a role in atherosclerosis development. In line with this hypothesis, thrombin induced PAR-1 activation can lead to VSMC migration and proliferation (Figure 2b). Moreover, thrombin elevates collagen production by VSMCs in a PAR-1 dependent manner [73], indicating a possible role for thrombin in plaque stability. Another feature of atherosclerotic plaque development is apoptosis. VSMCs that undergo apoptosis can generate thrombin via a process that accelerates the assembly of the prothrombinase complex [74]. In addition, thrombin generation is associated with vascular dabigatran was shown to attenuate atherosclerosis development [77] and promote plaque stability calcification [75].

Of note, rodents lack PAR-1 expression on platelets [71]. Therefore, experiments targeting PAR-1 in rodents can be directly ascribed to PAR-1 expressed by the vessel wall. Indeed, vascular injury is altered in PAR-1 deficient mice possibly via extracellular matrix formation and remodeling [72]. In line with these results, in vitro stimulation of VSMCs by thrombin stimulates extracellular matrix production [70].

Dabigatran inhibits thrombin mediated PAR1 function by inhibiting N-terminal cleavage and internalization [76]. The inhibition of thrombin activity by dabigatran may thus inhibit the development of cardiovascular disease by reducing pro-inflammatory signaling 


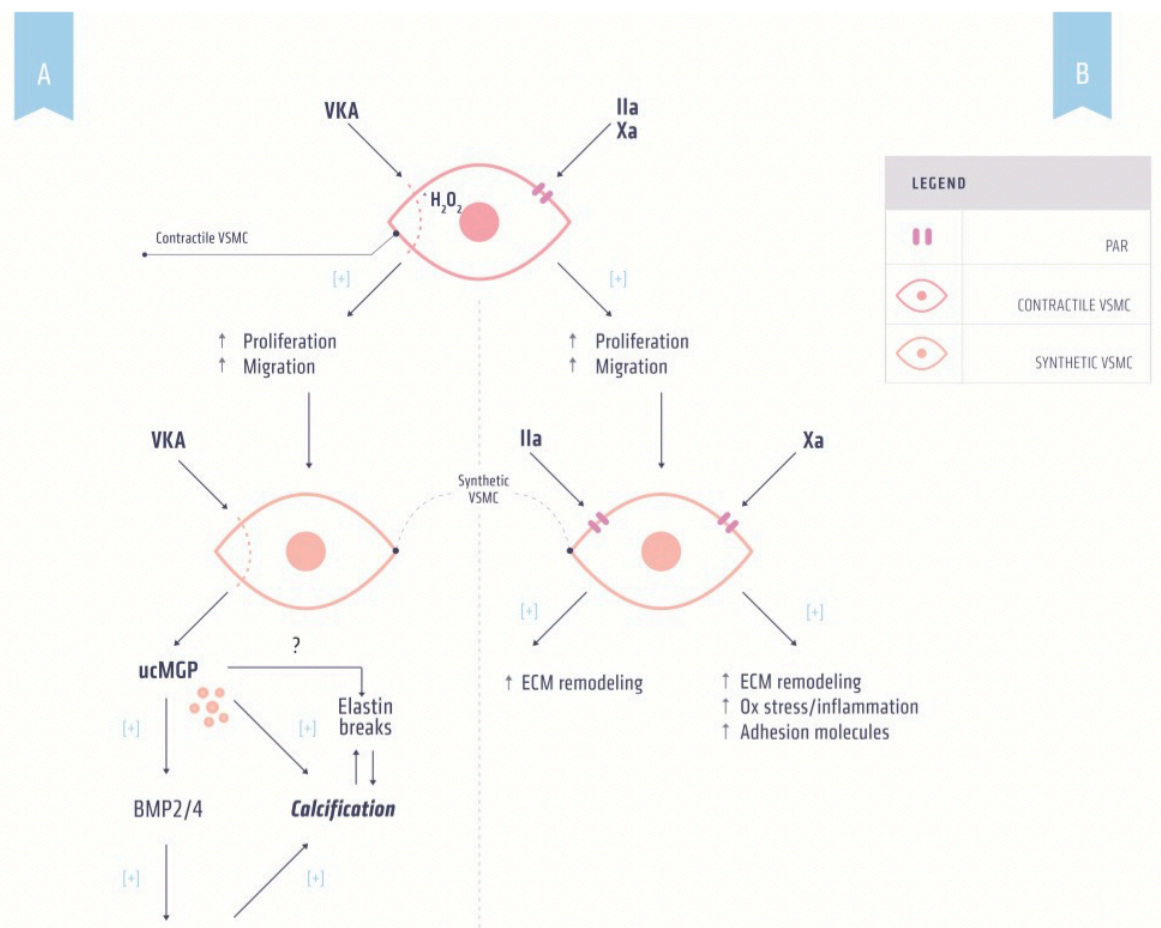

VSMC osteochondrogenic differentiation
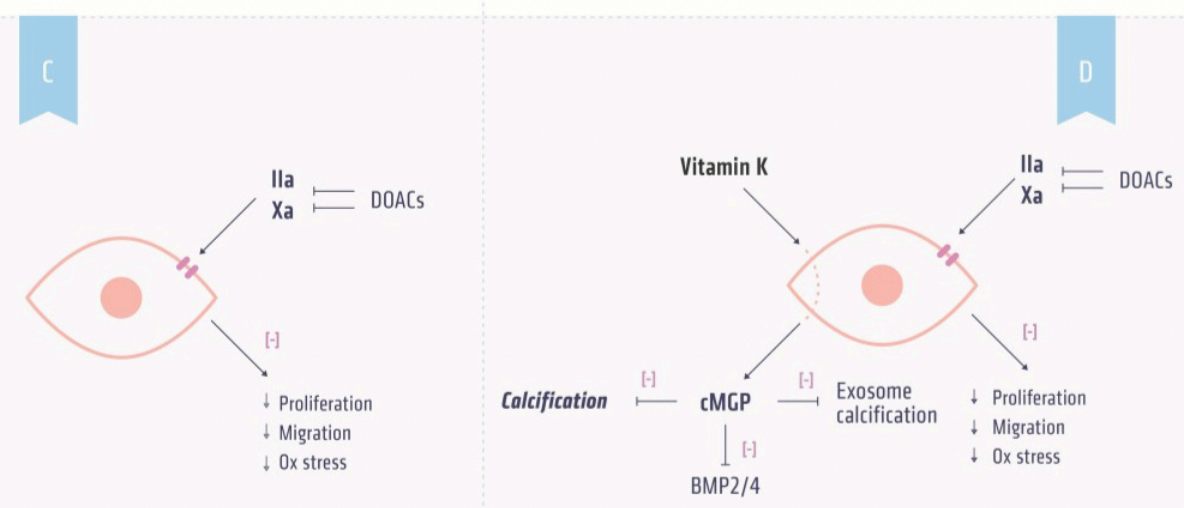

Figure 2. Effects of anticoagulants and vitamin $\mathrm{K}$ on vascular calcification. (A) Mechanism by which vitamin $\mathrm{K}$ antagonist (VKA) induces calcification. VKA induces phenotypic switching of contractile to synthetic vascular smooth muscle cells (VSMCs) by increasing oxidative stress resulting in increased vitamin K antagonist (VKA) induces calcification. VKA induces phenotypic switching of contractile proliferation and migration. Synthetic VSMCs secrete uncarboxylated matrix Gla protein (ucMGP) to synthetic vascular smooth muscle cells (VSMCs) by increasing oxidative stress resulting in as a result of vitamin K depletion induced by VKA. ucMGP is unable to inhibit bone morphogenetic increased proliferation and migration. Synthetic VSMCs secrete uncarboxylated matrix Gla protein protein (BMP) 2 and 4, a marker for osteochondrogenic differentiation. Osteochondrogenic VSMCs (ucMGP) as a result of vitamin $\mathrm{K}$ depletion induced by VKA. ucMGP is unable to inhibit bone are prone to calcification. Additionally, ucMGP is directly associated with increased calcification; morphogenetic protein (BMP) 2 and 4, a marker for osteochondrogenic differentiation. (B) Thrombin and factor Xa induce non-hemostasis 
signaling via protease-activated receptors (PARs). Osteochondrogenic VSMCs are prone to calcification. Additionally, ucMGP is directly associated Activation of PARs on contractile VSMCs can induce phenotypic switching resulting in increased proliferation and migration. PAR signaling in these synthetic VSMCs increases oxidative stress and adhesion molecules and induces extracellular (ECM) remodeling thereby facilitating calcification; (C) NOAC treatment in combination with (D) supplemental vitamin K administration has the potential to prevent both hypercoagulability and inhibit vascular calcification.

[67]. Indeed, dabigatran was shown to attenuate atherosclerosis development [77] and promote plaque stability [78].

\subsection{Factor Xa and Atherosclerosis}

Like thrombin, FXa induces non-hemostatic signaling via PARs. However, in contrast to thrombin FXa interacts only with PAR-1 and PAR-2 [70, 79]. As with PAR-1, the expression of PAR-2 is upregulated in human vascular lesions [70, 80]. FXa has been linked to pathophysiological conditions, including inflammation, tissue fibrosis and vascular remodeling [81]. In line with this link, FXa has been shown to induce inflammatory signaling and increase expression of cell adhesion molecules [67]. Moreover, FXa induces proliferation and migration of VSMCs via activation of PAR-2 thereby altering the composition and accumulation of extracellular matrix [82, 83]. The in vivo importance of PAR-2 in the inflammation process is suggested by findings that PAR-2 deficient mice display lower inflammation in a model of arthritis [84] and from rat studies in which lipopolysaccharide and oxidative stress increased the expression of PAR-2 [85, 86].

Taken together, the available data suggests that FXa has a role in atherosclerosis via its interaction with PAR-1 and PAR-2. Recently, treatment of atherosclerosis prone apoE ${ }^{-/-}$ mice with rivaroxaban resulted in increased plaque stability [87]. Since FXa activates prothrombin it is tempting to speculate that inhibition of FXa also prevents thrombinmediated effects in atherothrombosis.

\section{Vitamin K Dependent Proteins and Calcification}

Originally, vascular calcification was thought to be a passive process. The discovery of calcification inhibitors that actively prevent vascular calcification showed that it is a highly regulated process involving proteins and cellular components. The VKDP matrix Gla protein (MGP) is a local calcification inhibitor associated with calcifications in human lesions [88, 89]. Other VKDP associated with vascular calcification are osteocalcin (OC) and the more recently discovered Gla Rich Protein (GRP) [90]. Moreover, MGP, OC and the downstream regulator bone morphogenetic proteins (BMP) 2 and 4 are associated with microcalcifications in early human atherosclerotic lesions [88]. Microcalcifications in atherosclerosis are associated with increased plaque vulnerability [91-94].

\subsection{Osteocalcin}

Like all VKDP proteins, OC requires vitamin $\mathrm{K}$ for the $\gamma$-glutamylcarboxylation of three glutamate-residues, which in turn confers functional protein activity. In tissues and the 


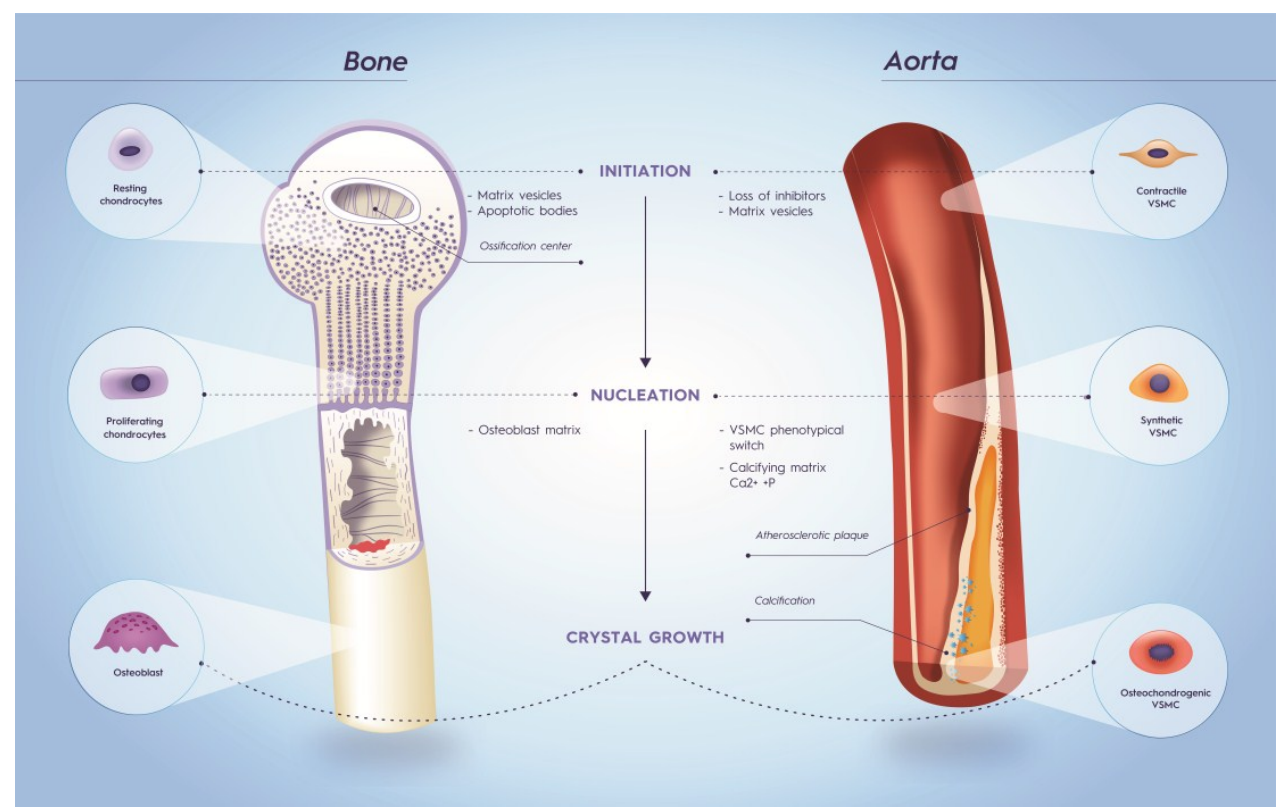

Figure 3. Similarities in bone metabolism and vascular calcification. The calcification process can be divided into three stages: initiation, nucleation and crystal growth. In order to initiate mineralization resting chondrocytes and contractile vascular smooth muscle cells (VSMCs) lose calcification inhibitors. Moreover, vesicles derived from chondrocytes and VSMCs form a nidus for calcification. In both bone metabolism and vascular calcification the matrix plays an important role in the nucleation stage. In bone metabolism, an osteoblast matrix results in proliferation of chondrocytes. Likewise, a calcifying matrix consisting of elastin, collagen and $\mathrm{Ca}^{2+}$ and $\mathrm{P}$ accompany vascular calcification. Additionally, contractile VSMCs undergo phenotypic switching resulting in synthetic VSMCs, which have increased proliferation and migration in comparison to contractile VSMCs. Finally, osteoblasts and osteochondrogenic VSMCs induce crystal growth in bone metabolism and vascular calcification, respectively.

circulation $\mathrm{OC}$ is present in both the carboxylated (cOC) and uncarboxylated (ucOC) conformations. OC is mostly associated with bone metabolism [95] where it promotes bone growth [96-98]. The processes of bone metabolism and soft tissue calcification are closely related, suggesting a possible role of osteocalcin in vascular calcification (Figure 3). As in bone metabolism, OC is thought to both promote [99] and inhibit [100-103] soft tissue calcification in order to regulate remodeling and mineralization [104]. It has been suggested that the inhibitory effect of $\mathrm{OC}$ on calcification is via mechanisms that prevent calcium and phosphate precipitation [17]. The role of $\mathrm{OC}$ in vascular calcification suggests the possibility that $\mathrm{OC}$ measurements can be used as a biomarker of calcification. OC-positive endothelial progenitor cells are elevated in patients with a history of cardiovascular events and were associated with calcification of coronary arteries $[105,106]$. During atherosclerosis, VSMCs undergo phenotypic switching resulting in osteoblast-like VSMCs, which are prone to calcification. Calcifying VSMCs express OC [107] and thus increased circulating concentrations of $\mathrm{OC}$ may reflect vascular calcification. Indeed, calcification of osteoblastlike VSMCs is associated with OC synthesis [108]. Furthermore, circulating levels of ucOC 
could provide insights into the relationship between vitamin $\mathrm{K}$ status and calcification. Indeed, it has been shown that ucOC concentrations are an independent predictor of carotid artery calcification [109].

\subsection{Matrix Gla Protein}

MGP is found in a wide range of tissues including heart, lungs, skin and the vasculature. In the vessel wall MGP is secreted by VSMCs [110]. Besides posttranslational carboxylation, MGP can also undergo serine phosphorylation. The precise role of the latter modification is not fully understood, but is thought to play a role in the secretion of MGP [111].

The pivotal role of MGP became clear from MGP-deficient mice, which all died within eight weeks after birth due to rupture of severely calcified arteries [112]. Analyses of the arteries revealed fragmented and calcified elastic fibers and the presence of osteochondrogenic-like cells. Surprisingly, the calcification phenotype of $\mathrm{MGP}^{-/-}$mice was not rescued by restoring circulating levels of MGP via hepatic MGP expression [113]. In contrast, restoring MGP expression in VSMCs rescued the vascular phenotype completely [113]. These data demonstrated that only VSMCs synthesis of carboxylated MGP (cMGP) is able to inhibit vascular calcification. MGP deficiency is also present in humans resulting in Keutel syndrome. Patients with Keutel syndrome suffer from abnormal soft tissue calcification [104] and have low levels of circulating cMGP [114].

Administration of warfarin results in similar calcification of arteries as observed in $\mathrm{MGP}^{-/-}$mice suggesting that warfarin-induced calcification is via impairment of MGP function [115]. Additionally, since warfarin chemically knocks down $\gamma$-carboxylated MGP it can be used as a model to investigate the role of MGP in vascular calcification [115].

There are several routes by which cMGP may inhibit calcification [110]. MGP is present in matrix vesicles and apoptopic bodies released from VSMCs [116]. In the presence of increased extracellular calcium MGP levels initially increase. However, when calcium levels are chronically elevated, MGP levels decrease [110]. Furthermore, MGP blocks VSMC phenotypic switching. Under physiological conditions, VSMCs display a contractile phenotype supporting vascular tone, and are not prone to calcify. In contrast, VSMCs undergoing synthetic or osteochondrogenic differentiation are susceptible to vascular calcification [17]. $\mathrm{MGP}^{-/-}$mice had decreased VSMC contractile markers and increased osteochondrogenic markers [112, 117]. The phenotypic switching of VSMCs is under the regulation of BMP 2 and 4, which in turn are inhibited by cMGP [118, 119]. MGP also directly inhibits calcium crystal growth by its ability to block nucleation sites through the binding of the negatively charged Gla domain and phosphorylated serine residues of MGP with growing hydroxyapatite crystals $[120,121]$. Finally, MGP prevents mineralization of elastin fibers by shedding nucleation sites, this process being facilitated by the low molecular weight and small size of MGP allowing it to prevent mineralization within the elastin fibers $[122,123]$. 


\subsection{Gla Rich Protein}

GRP, also termed Upper zone of growth plate and Cartilage Matrix Associated protein (UCMA), is a recently discovered VKDP highly conserved in animals and humans and involved in the inhibition of vascular calcification. Human gene expression and protein accumulation of GRP was shown in the fetal growth plate, vascular system and skin [124]. In human pathological conditions, GRP expression is associated with calcification of skin and arteries [90]. GRP has, like MGP, calcium-binding properties and acts as a calcification inhibitor [125]. The calcification-inhibitory effect of GRP is dose-dependent, requires $\gamma$ glutamylcarboxylation, and is thought to act via inhibition of osteochondrogenic switching of VSMCs [125]. GRP knockout mice, however lack phenotypic alterations [126].

\section{Role for Vitamin K in Cardiovascular Disease}

The first evidence that vitamin $\mathrm{K}$ is associated with vascular health came from data from the Rotterdam study [127]. In this observational study the risk for cardiovascular disease was some $50 \%$ lower in people in the highest tertile for menaquinone dietary intake (MK4 through MK10) [127]. In epidemiological studies, dietary intakes of menaquinones (MK-4 through MK-10) have been reported to be associated with a reduced risk for cardiovascular mortality [127, 128]. Additionally, higher menaquinone intakes (MK-4 through MK-10) or supplementation with MK-7 were associated with reduced calcification, which is presumed to be due to the improved $\gamma$-carboxylation and greater functional activity of VKDP [129, 130]. Finally, the long chain menaquinone isoprenologue MK-7 has been shown to have a greater impact on restoring coagulation compared to phylloquinone in VKA treated healthy volunteers indicating the overall better efficacy of MK-7 for the $\gamma$ carboxylation of the coagulation VKDP synthesized in the liver [14, 131]. Explanations for the putative beneficial effect of MK-7 on the vascular system for the $\gamma$-carboxylation of MGP and GRP is that long chain menaquinones such as MK7 are mainly transported via lowdensity lipoproteins and have a slower clearance rate from the circulation [13] as well as a higher co-factor activity for the $\gamma$ glutamyl-carboxylase [132].

In order to investigate the effect of vitamin $\mathrm{K}$ on the vascular system in humans a biomarker that reflects vitamin K status is required. Since vitamin K is essential for carboxylation of VKDP, the carboxylation status of VKDP can be used as a biomarker for vitamin $\mathrm{K}$ status. Dialysis patients have significantly increased levels of circulating uncarboxylated MGP (ucMGP) and reduced levels of cMGP indicative of a subclinical vascular vitamin K deficiency [133]. Moreover, circulating levels of ucMGP are positively associated with vascular calcification. Taken together, ucMGP seems a promising biomarker for vascular vitamin $\mathrm{K}$ status in relation to vascular calcification [89, 133-135]. Therefore, clinical studies investigating the effect of vitamin $\mathrm{K}$ on the vascular system and calcification use the carboxylation status of MGP as a biomarker [130]. Currently, clinical trials are ongoing to assess the effect of high intake of vitamin $\mathrm{K}$ on vascular calcification progression 
[136]. In these clinical studies both phylloquinone and menaquinones are under investigation and can therefore provide novel insights into the differential effect of vitamin $\mathrm{K}$ forms on vascular calcification.

\section{Vitamin K and Direct oral Anticoagulation}

Treating patients with hypercoagulability and vascular disease requires personalized medicine. Whereas both VKA and NOACs are equally suitable for treating hypercoagulability, VKA induces vascular calcification thereby affecting the vessel wall in a negative way (Figure 2a). Effects of NOACs on vascular calcification are not known yet, but are unlikely to affect VKDP activity (Figure 2b) [137]. Moreover, high intake of vitamin $\mathrm{K}$ has shown to inhibit and even reverse warfarin-induced vascular calcification in experimental animals [56, 138] and in adenine treated rats [52]. It is tempting to speculate that co-administration of vitamin $\mathrm{K}$ with anticoagulation therapy can target both coagulation and calcification. Since this co-administration is unsuitable with VKA [131], it should be investigated whether combining NOACs and vitamin $\mathrm{K}$ can be beneficial for both coagulation and calcification (Figure 2c). Presently, clinical trials assessing these aspects of co-administration are being conducted and the results of these studies should provide novel insights into personalized anticoagulation therapy [136].

\section{Conclusions}

Currently, VKA are still the most widely prescribed drugs used for anticoagulation therapy. However, owing to the unfavorable pharmacokinetics and actions of VKA, direct thrombin and FXa inhibitors have been introduced as alternatives to VKA. Clinical studies have demonstrated that NOACs are non-inferior to VKA but are likely to lack the calcification-inducing side effect of VKA. Additionally, NOACs exert beneficial effects on atherogenesis via PAR signaling. Presently, ongoing clinical trials are addressing whether vitamin $\mathrm{K}$ supplementation can halt or regress vascular calcification. The outcome of these trials will pave the way to test whether co-supplementation of vitamin K with NOACs can benefit both coagulation and calcification.

Acknowledgments: This work was supported in part by research funding from the Trombose Stichting Nederland (grant 2014.02) and by the Norwegian Research Council and NattoPharma ASA.

Author Contributions: Rick van Gorp and Leon Schurgers wrote the manuscript. Leon Schurgers had primary responsibility for the final content.

Conflicts of Interest: Nattopharma ASA received an industrial $\mathrm{PhD}$ grant from the Norwegian Research Council to conduct research on vitamin $\mathrm{K}$ in collaboration with the 
Maastricht University. Rick van Gorp has been employed as $\mathrm{PhD}$ student to work on this project. Nattopharma ASA is a pharmaceutical company with interest in vitamin $\mathrm{K}_{2}$.

\section{References}

1. Mann, K.G. Biochemistry and physiology of blood coagulation. Thromb. Haemost. 1999, 82, $165-174$.

2. Macfarlane, R.G. An enzyme cascade in the blood clotting mechanism, and its function as a biochemical amplifier. Nature 1964, 202, 498-499.

3. Colman, R.W. Are hemostasis and thrombosis two sides of the same coin? J. Exp. Med. 2006, $203,493-495$.

4. Badimon, L. Atherosclerosis and thrombosis: Lessons from animal models. Thromb. Haemost. 2001, 86, 356-365.

5. Fuster, V.; Stein, B.; Ambrose, J.A.; Badimon, L.; Badimon, J.J.; Chesebro, J.H. Atherosclerotic plaque rupture and thrombosis. Evolving concepts. Circulation 1990, 82, II47-II59.

6. Rosendaal, F.R. Venous thrombosis: A multicausal disease. Lancet 1999, 353, 1167-1173.

7. Schofield, F.W. A brief account of a disease in cattle simulating hemorrhagic septicaemia due to feeding sweet clover. Can. Vet. J. Rev. Vétérinaire Can. 1984, 25, 453-455.

8. Link, K. The discovery of dicumarol and its sequels. Circulation 1959, 19, 97-107.

9. Shapiro, S.; Ciferri, F.E. Intramuscular administration of the anticoagulant warfarin (coumadin) sodium. J. Am. Med. Assoc. 1957, 165, 1377-1380.

10. Beinema, M.; Brouwers, J.R.B.J.; Schalekamp, T.; Wilffert, B. Pharmacogenetic differences between warfarin, acenocoumarol and phenprocoumon. Thromb. Haemost. 2008, 100, 1052-1057.

11. Beulens, J.W.J.; Booth, S.L.; van den Heuvel, E.G.H.M.; Stoecklin, E.; Baka, A.; Vermeer, C. The role of menaquinones (vitamin K2) in human health. Br. J. Nutr. 2013, 110, 1357-1368.

12. Schurgers, L.J.; Vermeer, C. Determination of phylloquinone and menaquinones in food. Eff. Food Matrix Circ. Vitam. K Conc. 2000, 30, 298-307.

13. Schurgers, L.J.; Vermeer, C. Differential lipoprotein transport pathways of K-vitamins in healthy subjects. Biochim. Biophys. Acta 2002, 1570, 27-32.

14. Schurgers, L.J.; Teunissen, K.J.F.; Hamulyak, K.; Knapen, M.H.J.; Vik, H.; Vermeer, C. Vitamin K-containing dietary supplements: Comparison of synthetic vitamin K1 and natto-derived menaquinone-7. Blood 2007, 109, 3279-3283.

15. Thijssen, H.H.W.; Vervoort, L.M.T.; Schurgers, L.J.; Shearer, M.J. Menadione is a metabolite of oral vitamin K. Br. J. Nutr. 2006, 95, 260-266.

16. Esmon, C.; Sadowski, J.; Suttie, J. A new carboxylation reaction. The vitamin K-dependent incorporation of H-14CO3- into prothrombin. J. Biol. Chem. 1975, 250, 4744-4748.

17. Willems, B.A.G.; Vermeer, C.; Reutelingsperger, C.P.M.; Schurgers, L.J. The realm of vitamin K dependent proteins: Shifting from coagulation toward calcification. Mol. Nutr. Food Res. 2014, 58, 1620-1635.

18. Van Horn, W.D. Structural and functional insights into human vitamin K epoxide reductase and vitamin K epoxide reductase-like1. Crit. Rev. Biochem. Mol. Biol. 2013, 48, 357-372.

19. Stafford, D. The vitamin K cycle. J. Thromb. Haemost. 2005, 3, 1873-1878.

20. Weitz, J.I.; Hirsh, J. New antithrombotic agents. Chest 1998, 114, 715S-727S.

21. Van Aken, H.; Bode, C.; Darius, H.; Diehm, C.; Encke, A.; Gulba, D.C.; Haas, S.; Hacke, W.; Puhl, W.; Quante, M.; et al. State-of-the-art review: Anticoagulation: The present and future. Clin. Appl. Thromb. Hemost. 2001, 7 , 195-204.

22. Greinacher, A.; Warkentin, T.E. The direct thrombin inhibitor hirudin. Thromb. Haemost. 2008, 99, 819-829.

23. Eriksson, B.I.; Wille-Jørgensen, P.; Kälebo, P.; Mouret, P.; Rosencher, N.; Bösch, P.; Baur, M.; Ekman, S.; Bach, D.; Lindbratt, S.; et al. A comparison of recombinant hirudin with a low-molecular-weight heparin to prevent thromboembolic complications after total hip replacement. N. Engl. J. Med. 1997, 337, 1329-1335.

24. Investigators, G.L. Randomized trial of intravenous heparin versus recombinant hirudin for acute coronary syndromes. The Global Use of Strategies to Open Occluded Coronary Arteries (GUSTO) IIa Investigators. Circulation 1994, 90, 1631-1637.

25. Schulman, S.; Kearon, C.; Kakkar, A.K.; Mismetti, P.; Schellong, S.; Eriksson, H.; Baanstra, D.; Schnee, J.; Goldhaber, S.Z. RE-COVER Study Group Dabigatran versus warfarin in the treatment of acute venous thromboembolism. N. Engl. J. Med. 2009, 361, 2342-2352.

26. Schulman, S.; Wåhlander, K.; Lundström, T.; Clason, S.B.; Eriksson, H. THRIVE III Investigators Secondary prevention of venous thromboembolism with the oral direct thrombin inhibitor ximelagatran. N. Engl. J. Med. 2003, 349, 1713-1721.

27. Laizure, S.C.; Parker, R.B.; Herring, V.L.; Hu, Z.-Y. Identification of carboxylesterase-dependent dabigatran etexilate hydrolysis. Drug Metab. Dispos. Biol. Fate Chem. 2014, 42, 201-206. 
28. Akwaa, F.; Spyropoulos, A.C. The potential of target-specific oral anticoagulants for the acute and long-term treatment of venous thromboembolism. Curr. Med. Res. Opin. 2014, 30, 2179-2190.

29. Yeh, C.H.; Hogg, K.; Weitz, J.I. Overview of the New Oral Anticoagulants: Opportunities and Challenges. Arterioscler. Thromb. Vasc. Biol. 2015, 35, 1056-1065.

30. Kubitza, D.; Becka, M.; Wensing, G.; Voith, B.; Zuehlsdorf, M. Safety, pharmacodynamics, and pharmacokinetics of BAY 59-7939-An oral, direct Factor Xa inhibitor-After multiple dosing in healthy male subjects. Eur. J. Clin. Pharmacol. 2005, 61, 873-880.

31. Kubitza, D.; Haas, S. Novel factor Xa inhibitors for prevention and treatment of thromboembolic diseases. Expert Opin. Investig. Drugs 2006, 15, 843-855.

32. Weitz, J.I. Emerging anticoagulants for the treatment of venous thromboembolism. Thromb. Haemost. 2006, 96, 274-284.

33. Nutt, E.M.; Jain, D.; Lenny, A.B.; Schaffer, L.; Siegl, P.K.; Dunwiddie, C.T. Purification and characterization of recombinant antistasin: A leech-derived inhibitor of coagulation factor Xa. Arch. Biochem. Biophys. 1991, 285, 37 44.

34. Yeh, C.H.; Fredenburgh, J.C.; Weitz, J.I. Oral direct factor Xa inhibitors. Circ. Res. 2012, 111, 1069-1078.

35. Perzborn, E.; Strassburger, J.; Wilmen, A.; Pohlmann, J.; Roehrig, S.; Schlemmer, K.-H.; Straub, A. In vitro and in vivo studies of the novel antithrombotic agent BAY 59-7939-An oral, direct Factor Xa inhibitor. J. Thromb. Haemost. 2005, 3, 514-521.

36. Schurgers, L.J.; Spronk, H.M.H. Differential cellular effects of old and new oral anticoagulants: Consequences to the genesis and progression of atherosclerosis. Thromb. Haemost. 2014, 112, 909-917.

37. Kreutz, R. A clinical and pharmacologic assessment of once-daily versus twice-daily dosing for rivaroxaban. $J$. Thromb. Thrombolysis 2014, 38, 137-149.

38. Barritt, D.W.; Jordan, S.C. Anticoagulant drugs in the treatment of pulmonary embolism. A controlled trial. Lancet 1960, 1, 1309-1312.

39. Duxbury, B.M.; Poller, L. The oral anticoagulant saga: Past, present, and future. Clin. Appl. Thromb. Hemost. 2001, 7, 269-275.

40. Hull, R.; Hirsh, J.; Jay, R.; Carter, C.; England, C.; Gent, M.; Turpie, A.G.; McLoughlin, D.; Dodd, P.; Thomas, M.; et al. Different intensities of oral anticoagulant therapy in the treatment of proximal-vein thrombosis. $N$. Engl. J. Med. 1982, 307, 1676-1681.

41. Guyatt, G.H.; Akl, E.A.; Crowther, M.; Gutterman, D.D.; Schuünemann, H.J. Executive summary: Antithrombotic therapy and prevention of thrombosis, 9 th ed.: American college of chest physicians evidence-based clinical practice guidelines. Chest 2012, 141, 7S-47S.

42. Connolly, S.J.; Ezekowitz, M.D.; Yusuf, S.; Eikelboom, J.; Oldgren, J.; Parekh, A.; Pogue, J.; Reilly, P.A.; Themeles, E.; Varrone, J.; et al. RE-LY Steering Committee and Investigators Dabigatran versus warfarin in patients with atrial fibrillation. N. Engl. J. Med. 2009, 361, 1139-1151.

43. Eriksson, B.I.; Dahl, O.E.; Rosencher, N.; Kurth, A.A.; van Dijk, C.N.; Frostick, S.P.; Kälebo, P.; Christiansen, A.V.; Hantel, S.; Hettiarachchi, R.; et al. RE-MODEL Study Group Oral dabigatran etexilate vs. subcutaneous enoxaparin for the prevention of venous thromboembolism after total knee replacement: The RE-MODEL randomized trial. J. Thromb. Haemost. 2007, 5, 2178-2185.

44. Patel, M.R.; Mahaffey, K.W.; Garg, J.; Pan, G.; Singer, D.E.; Hacke, W.; Breithardt, G.; Halperin, J.L.; Hankey, G.J.; Piccini, J.P.; et al. ROCKET AF Investigators Rivaroxaban versus warfarin in nonvalvular atrial fibrillation. N. Engl. J. Med. 2011, 365, 883-891.

45. Eriksson, B.I.; Borris, L.C.; Friedman, R.J.; Haas, S.; Huisman, M.V.; Kakkar, A.K.; Bandel, T.J.; Beckmann, H.; Muehlhofer, E.; Misselwitz, F.; et al. RECORD1 Study Group Rivaroxaban versus enoxaparin for thromboprophylaxis after hip arthroplasty. N. Engl. J. Med. 2008, 358, 2765-2775.

46. EINSTEIN Investigators; Bauersachs, R.; Berkowitz, S.D.; Brenner, B.; Büller, H.R.; Decousus, H.; Gallus, A.S.; Lensing, A.W.; Misselwitz, F.; Prins, M.H.; et al. Oral rivaroxaban for symptomatic venous thromboembolism. $N$. Engl. J. Med. 2010, 363, 2499-2510.

47. Eriksson, B.I.; Kakkar, A.K.; Turpie, A.G.G.; Gent, M.; Bandel, T.-J.; Homering, M.; Misselwitz, F.; Lassen, M.R. Oral rivaroxaban for the prevention of symptomatic venous thromboembolism after elective hip and knee replacement. J. Bone Joint Surg. Br. 2009, 91, 636-644.

48. Granger, C.B.; Alexander, J.H.; McMurray, J.J.V.; Lopes, R.D.; Hylek, E.M.; Hanna, M.; Al-Khalidi, H.R.; Ansell, J.; Atar, D.; Avezum, A.; et al. ARISTOTLE Committees and Investigators Apixaban versus warfarin in patients with atrial fibrillation. N. Engl. J. Med. 2011, 365, 981-992.

49. Lassen, M.R.; Gallus, A.; Raskob, G.E.; Pineo, G.; Chen, D.; Ramirez, L.M. ADVANCE-3 Investigators Apixaban versus enoxaparin for thromboprophylaxis after hip replacement. N. Engl. J. Med. 2010, 363, 2487-2498.

50. Van Es, N.; Coppens, M.; Schulman, S.; Middeldorp, S.; Büller, H.R. Direct oral anticoagulants compared with vitamin $\mathrm{K}$ antagonists for acute venous thromboembolism: Evidence from phase 3 trials. Blood 2014, 124, 19681975 . 
51. Haas, S. Medical indications and considerations for future clinical decision making. Thromb. Res. 2003,109 (Suppl. 1), S31-S37.

52. McCabe, K.M.; Booth, S.L.; Fu, X.; Shobeiri, N.; Pang, J.J.; Adams, M.A.; Holden, R.M. Dietary vitamin K and therapeutic warfarin alter the susceptibility to vascular calcification in experimental chronic kidney disease. Kidney Int. 2013, 83, 835-844.

53. Chatrou, M.L.L.; Winckers, K.; Hackeng, T.M.; Reutelingsperger, C.P.; Schurgers, L.J. Vascular calcification: The price to pay for anticoagulation therapy with vitamin K-antagonists. Blood Rev. 2012, 26, 155-166.

54. Rosenhek, R.; Binder, T.; Porenta, G.; Lang, I.; Christ, G.; Schemper, M.; Maurer, G.; Baumgartner, H. Predictors of outcome in severe, asymptomatic aortic stenosis. N. Engl. J. Med. 2000, 343, 611-617.

55. Rennenberg, R.J.M.W.; Kessels, A.G.H.; Schurgers, L.J.; van Engelshoven, J.M.A.; de Leeuw, P.W.; Kroon, A.A. Vascular calcifications as a marker of increased cardiovascular risk: A meta-analysis. Vasc. Health Risk Manag. 2009, 5, 185-197.

56. Schurgers, L.J.; Spronk, H.M.; Soute, B.A.; Schiffers, P.; DeMey, J.G.R.; Vermeer, C. Regression of warfarininduced medial elastocalcinosis by high intake of vitamin K in rats. Blood 2007, 109, 2823-2831.

57. Mac-Way, F.; Poulin, A.; Utescu, M.S.; De Serres, S.A.; Marquis, K.; Douville, P.; Desmeules, S.; Larivière, R.; Lebel, M.; Agharazii, M. The impact of warfarin on the rate of progression of aortic stiffness in hemodialysis patients: A longitudinal study. Nephrol. Dial. Transplant. 2014, 29, 2113-2120.

58. Cohen, D. Dabigatran: How the drug company withheld important analyses. BMJ (Clin. Res. ed.) $2014,349$.

59. Moore, T.J.; Cohen, M.R.; Mattison, D.R. Dabigatran, bleeding, and the regulators. BMJ (Clin. Res. ed.) 2014, 349.

60. Favaloro, E.J.; Lippi, G. Laboratory testing in the era of direct or non-vitamin K antagonist oral anticoagulants: A practical guide to measuring their activity and avoiding diagnostic errors. Semin. Thromb. Hemost. 2015, 41, 208227.

61. Lippi, G.; Favaloro, E.J.; Mattiuzzi, C. Combined administration of antibiotics and direct oral anticoagulants: A renewed indication for laboratory monitoring? Semin. Thromb. Hemost. 2014, 40, 756-765.

62. Bauer, K.A. Targeted Anti-Anticoagulants. N. Engl. J. Med. 2015, 373, 569-571.

63. Shearer, M.J.; Newman, P. Metabolism and cell biology of vitamin K. Thromb. Haemost. 2008, 100, 530-547.

64. Crowther, M.; Crowther, M.A. Antidotes for Novel Oral Anticoagulants: Current Status and Future Potential. Arterioscler. Thromb. Vasc. Biol. 2015, 35, 1736-1745.

65. Pollack, C.V.; Reilly, P.A.; Eikelboom, J.; Glund, S.; Verhamme, P.; Bernstein, R.A.; Dubiel, R.; Huisman, M.V.; Hylek, E.M.; Kamphuisen, P.W.; et al. Idarucizumab for Dabigatran Reversal. N. Engl. J. Med. 2015, 373, 511-520.

66. Patterson, C.; Stouffer, G.A.; Madamanchi, N.; Runge, M.S. New tricks for old dogs: Nonthrombotic effects of thrombin in vessel wall biology. Circ. Res. 2001, 88, 987-997.

67. Borissoff, J.I.; Spronk, H.M.H.; Ten Cate, H. The hemostatic system as a modulator of atherosclerosis. N. Engl. J. Med. 2011, 364, 1746-1760.

68. Ma, L.; Dorling, A. The roles of thrombin and protease-activated receptors in inflammation. Semin. Immunopathol. 2012, 34, 63-72.

69. Kalz, J.; Ten Cate, H.; Spronk, H.M.H. Thrombin generation and atherosclerosis. J. Thromb. Thrombolysis 2014, $37,45-55$.

70. Alberelli, M.A.; De Candia, E. Functional role of protease activated receptors in vascular biology. Vasc. Pharmacol. 2014, 62, 72-81.

71. Martorell, L.; Martínez-González, J.; Rodríguez, C.; Gentile, M.; Calvayrac, O.; Badimon, L. Thrombin and protease-activated receptors (PARs) in atherothrombosis. Thromb. Haemost. 2008, 99, 305-315.

72. Cheung, W.M.; D'Andrea, M.R.; Andrade-Gordon, P.; Damiano, B.P. Altered vascular injury responses in mice deficient in protease-activated receptor-1. Arterioscler. Thromb. Vasc. Biol. 1999, 19, 3014-3024.

73. Dabbagh, K.; Laurent, G.J.; McAnulty, R.J.; Chambers, R.C. Thrombin stimulates smooth muscle cell procollagen synthesis and mRNA levels via a PAR-1 mediated mechanism. Thromb. Haemost. 1998, 79, 405-409.

74. Flynn, P.; Byrne, C.; Baglin, T.; Weissberg, P.; Bennett, M. Thrombin generation by apoptotic vascular smooth muscle cells. Blood 1997, 89, 4378-4384.

75. Borissoff, J.I.; Joosen, I.A.; Versteylen, M.O.; Spronk, H.M.; ten Cate, H.; Hofstra, L. Accelerated In Vivo Thrombin Formation Independently Predicts the Presence and Severity of CT Angiographic Coronary Atherosclerosis. JACC Cardiovasc. Imaging 2012, 5, 1201-1210.

76. Chen, B.; Soto, A.G.; Coronel, L.J.; Goss, A.; van Ryn, J.; Trejo, J. Characterization of Thrombin-Bound Dabigatran Effects on Protease-Activated Receptor-1 Expression and Signaling In Vitro. Mol. Pharmacol. 2015, 88, 95-105.

77. Pingel, S.; Tiyerili, V.; Mueller, J.; Werner, N.; Nickenig, G.; Mueller, C. Thrombin inhibition by dabigatran attenuates atherosclerosis in ApoE deficient mice. Arch. Med. Sci. 2014, 10, 154-160.

78. Kadoglou, N.P.E.; Moustardas, P.; Katsimpoulas, M.; Kapelouzou, A.; Kostomitsopoulos, N.; Schafer, K.; Kostakis, A.; Liapis, C.D. The beneficial effects of a direct thrombin inhibitor, dabigatran etexilate, on the development and stability of atherosclerotic lesions in apolipoprotein E-deficient mice: Dabigatran etexilate and atherosclerosis. Cardiovasc. Drugs Ther. Spons. Int. Soc. Cardiovasc. Pharmacother. 2012, 26, 367-374. 
79. Borensztajn, K.; Peppelenbosch, M.P.; Spek, C.A. Factor Xa: At the crossroads between coagulation and signaling in physiology and disease. Trends Mol. Med. 2008, 14, 429-440.

80. Lee, H.; Hamilton, J.R. Physiology, pharmacology, and therapeutic potential of protease-activated receptors in vascular disease. Pharmacol. Ther. 2012, 134, 246-259.

81. Esmon, C.T. Targeting factor Xa and thrombin: Impact on coagulation and beyond. Thromb. Haemost. 2014, 111, $625-633$.

82. Böhm, A.; Flößer, A.; Ermler, S.; Fender, A.C.; Lüth, A.; Kleuser, B.; Schrör, K.; Rauch, B.H. Factor-Xa-induced mitogenesis and migration require sphingosine kinase activity and S1P formation in human vascular smooth muscle cells. Cardiovasc. Res. 2013, 99, 505-513.

83. Borensztajn, K.; Spek, C.A. Blood coagulation factor Xa as an emerging drug target. Expert Opin. Ther. Targets 2011, 15, 341-349.

84. Ferrell, W.R.; Lockhart, J.C.; Kelso, E.B.; Dunning, L.; Plevin, R.; Meek, S.E.; Smith, A.J.H.; Hunter, G.D.; McLean, J.S.; McGarry, F.; et al. Essential role for proteinase-activated receptor-2 in arthritis. J. Clin. Investig. 2003, 111, 35-41.

85. Aman, M.; Hirano, M.; Kanaide, H.; Hirano, K. Upregulation of proteinase-activated receptor-2 and increased response to trypsin in endothelial cells after exposure to oxidative stress in rat aortas. J. Vasc. Res. 2010, 47, 494506.

86. Cicala, C.; Pinto, A.; Bucci, M.; Sorrentino, R.; Walker, B.; Harriot, P.; Cruchley, A.; Kapas, S.; Howells, G.L.; Cirino, G. Protease-activated receptor-2 involvement in hypotension in normal and endotoxemic rats in vivo. Circulation 1999, 99, 2590-2597.

87. Zhou, Q.; Bea, F.; Preusch, M.; Wang, H.; Isermann, B.; Shahzad, K.; Katus, H.A.; Blessing, E. Evaluation of plaque stability of advanced atherosclerotic lesions in apo E-deficient mice after treatment with the oral factor Xa inhibitor rivaroxaban. Mediat. Inflamm. 2011, 2011, 1-9.

88. Roijers, R.B.; Debernardi, N.; Cleutjens, J.P.M.; Schurgers, L.J.; Mutsaers, P.H.A.; van der Vusse, G.J. Microcalcifications in early intimal lesions of atherosclerotic human coronary arteries. Am. J. Pathol. 2011, 178, 2879-2887.

89. Schurgers, L.J.; Teunissen, K.J.F.; Knapen, M.H.J.; Kwaijtaal, M.; van Diest, R.; Appels, A.; Reutelingsperger, C.P.; Cleutjens, J.P.M.; Vermeer, C. Novel conformation-specific antibodies against matrix $\gamma$-carboxyglutamic acid (Gla) protein: Undercarboxylated matrix Gla protein as marker for vascular calcification. Arterioscler. Thromb. Vasc. Biol. 2005, 25, 1629-1633.

90. Viegas, C.S.B.; Cavaco, S.; Williamson, M.K.; Price, P.A.; Cancela, M.L.; Simes, D.C. Gla-rich protein is a novel vitamin K-dependent protein present in serum that accumulates at sites of pathological calcifications. Am. J. Pathol. 2009, 175, 2288-2298.

91. Joshi, N.V.; Vesey, A.T.; Williams, M.C.; Shah, A.S.V.; Calvert, P.A.; Craighead, F.H.M.; Yeoh, S.E.; Wallace, W.; Salter, D.; Fletcher, A.M.; et al. 18F-fluoride positron emission tomography for identification of ruptured and high-risk coronary atherosclerotic plaques: A prospective clinical trial. Lancet 2014, 383, 705-713.

92. Ehara, S.; Kobayashi, Y.; Yoshiyama, M.; Shimada, K.; Shimada, Y.; Fukuda, D.; Nakamura, Y.; Yamashita, H.; Yamagishi, H.; Takeuchi, K.; et al. Spotty calcification typifies the culprit plaque in patients with acute myocardial infarction: An intravascular ultrasound study. Circulation 2004, 110, 3424-3429.

93. Bluestein, D.; Alemu, Y.; Avrahami, I.; Gharib, M.; Dumont, K.; Ricotta, J.J.; Einav, S. Influence of microcalcifications on vulnerable plaque mechanics using FSI modeling. J. Biomech. 2008, 41, 1111-1118.

94. Vengrenyuk, Y.; Carlier, S.; Xanthos, S.; Cardoso, L.; Ganatos, P.; Virmani, R.; Einav, S.; Gilchrist, L.; Weinbaum, S. A hypothesis for vulnerable plaque rupture due to stress-induced debonding around cellular microcalcifications in thin fibrous caps. Proc. Natl. Acad. Sci. USA 2006, 103, 14678-14683.

95. Lombardi, G.; Perego, S.; Luzi, L.; Banfi, G. A four-season molecule: Osteocalcin. Updates in its physiological roles. Endocrine 2014, 48, 394-404.

96. Schlieper, G.; Schurgers, L.; Brandenburg, V.; Reutlingsperger, C.; Floege, J. Vascular calcification in chronic kidney disease: An update. Nephrol. Dial. Transplant. 2015.

97. Yamada, S.; Taniguchi, M.; Tokumoto, M.; Toyonaga, J.; Fujisaki, K.; Suehiro, T.; Noguchi, H.; Iida, M.; Tsuruya, K.; Kitazono, T. The antioxidant tempol ameliorates arterial medial calcification in uremic rats: Important role of oxidative stress in the pathogenesis of vascular calcification in chronic kidney disease. J. Bone Miner. Res. 2012, $27,474-485$.

98. Ndip, A.; Wilkinson, F.L.; Jude, E.B.; Boulton, A.J.; Alexander, M.Y. RANKL-OPG and RAGE modulation in vascular calcification and diabetes: Novel targets for therapy. Diabetologia 2014, 57, 2251-2260.

99. Lei, Y.; Sinha, A.; Nosoudi, N.; Grover, A.; Vyavahare, N. Hydroxyapatite and calcified elastin induce osteoblastlike differentiation in rat aortic smooth muscle cells. Exp. Cell Res. 2014, 323, 198-208.

100. Hunter, G.K.; Hauschka, P.V.; Poole, A.R.; Rosenberg, L.C.; Goldberg, H.A. Nucleation and inhibition of hydroxyapatite formation by mineralized tissue proteins. Biochem. J. 1996, 317, 59-64.

101. Ducy, P.; Desbois, C.; Boyce, B.; Pinero, G.; Story, B.; Dunstan, C.; Smith, E.; Bonadio, J.; Goldstein, S.; Gundberg, C.; et al. Increased bone formation in osteocalcin-deficient mice. Nature 1996, 382, 448-452. 
102. Kavukcuoglu, N.B.; Patterson-Buckendahl, P.; Mann, A.B. Effect of osteocalcin deficiency on the nanomechanics and chemistry of mouse bones. J. Mech. Behav. Biomed. Mater. 2009, 2, 348-354.

103. Roy, M.E.; Nishimoto, S.K.; Rho, J.Y.; Bhattacharya, S.K.; Lin, J.S.; Pharr, G.M. Correlations between osteocalcin content, degree of mineralization, and mechanical properties of C. carpio rib bone. J. Biomed. Mater. Res. 2001, 54, 547-553.

104. Evrard, S.; Delanaye, P.; Kamel, S.; Cristol, J.-P.; Cavalier, E. SFBC/SN joined working group on vascular calcifications vascular calcification: From pathophysiology to biomarkers. Clin. Chim. Acta 2015, 438, 401-414.

105. Price, P.A.; Otsuka, A.A.; Poser, J.W.; Kristaponis, J.; Raman, N. Characterization of a $\gamma$-carboxyglutamic acidcontaining protein from bone. Proc. Natl. Acad. Sci. USA 1976, 73, 1447-1451.

106. Van de Loo, P.G.F.; Soute, B.; van Haarlem, L.; Vermeer, C. The effect of Gla-containing proteins on the precipitation of insoluble salts. Biochem. Biophys. Res. Commun. 1987, 142, 113-119.

107. Kapustin, A.N.; Shanahan, C.M. Osteocalcin: A novel vascular metabolic and osteoinductive factor? Arterioscler. Thromb. Vasc. Biol. 2011, 31, 2169-2171.

108. Aikawa,E.;Nahrendorf,M.;Sosnovik,D.;Lok,V.M.;Jaffer,F.A.;Aikawa,M.;Weissleder,R.Multimodality molecular imaging identifies proteolytic and osteogenic activities in early aortic valve disease. Circulation 2007, 115, 377 386.

109. Okura, T.; Kurata, M.; Enomoto, D.; Jotoku, M.; Nagao, T.; Desilva, V.R.; Higaki, J. Undercarboxylated osteocalcin is a biomarker of carotid calcification in patients with essential hypertension. Kidney Blood Press. Res. 2010, 33, 66-71.

110. Schurgers, L.J.; Uitto, J.; Reutelingsperger, C.P. Vitamin K-dependent carboxylation of matrix Gla-protein: A crucial switch to control ectopic mineralization. Trends Mol. Med. 2013, 19, 217-226.

111. Wajih, N.; Borras, T.; Xue, W.; Hutson, S.M.; Wallin, R. Processing and transport of matrix $\gamma$-carboxyglutamic acid protein and bone morphogenetic protein-2 in cultured human vascular smooth muscle cells: Evidence for an uptake mechanism for serum fetuin. J. Biochem. 2004, 279, 43052-43060.

112. Luo,G.;Ducy,P.;McKee,M.;Pinero,G.;Loyer,E.;Behringer,R.;Karsenty,G.Spontaneouscalcificationof arteries and cartilage in mice lacking matrix GLA protein. Nature 1997, 385, 78-81.

113. Murshed, M.; Schinke, T.; McKee, M.D.; Karsenty, G. Extracellular matrix mineralization is regulatedlocally; different roles of two gla-containing proteins. J. Cell Biol. 2004, 165, 625-630.

114. Cranenburg, E.C.M.; van Spaendonck-Zwarts, K.Y.; Bonafe, L.; Mittaz Crettol, L.; Rödiger, L.A.; Dikkers, F.G.; van Essen, A.J.; Superti-Furga, A.; Alexandrakis, E.; Vermeer, C.; et al. Circulating matrix $\gamma$-carboxyglutamate protein (MGP) species are refractory to vitamin $\mathrm{K}$ treatment in a new case of Keutel syndrome. J. Thromb. Haemost. 2011, 9, 1225-1235.

115. Price, P.; Faus, S.; Williamson, M. Warfarin causes rapid calcification of the elastic lamellae in rat arteries and heart valves. Arterioscler. Thromb. Vasc. Biol. 1998, 18, 1400-1407.

116. Reynolds,J.L.;Joannides,A.J.;Skepper,J.N.;McNair,R.;Schurgers,L.J.;Proudfoot,D.;Jahnen-Dechent,W.; Weissberg, P.L.; Shanahan, C.M. Human vascular smooth muscle cells undergo vesicle-mediated calcification in response to changes in extracellular calcium and phosphate concentrations: A potential mechanism for accelerated vascular calcification in ESRD. J. Am. Soc. Nephrol. 2004, 15, 2857-2867.

117. Steitz, S.A.; Speer, M.Y.; Curinga, G.; Yang, H.Y.; Haynes, P.; Aebersold, R.; Schinke, T.; Karsenty, G.; Giachelli, C.M. Smooth muscle cell phenotypic transition associated with calcification: Upregulation of Cbfa1 and downregulation of smooth muscle lineage markers. Circ. Res. 2001, 89, 1147-1154.

118. Yao, Y.; Bennett, B.J.; Wang, X.; Rosenfeld, M.E.; Giachelli, C.; Lusis, A.J.; Boström, K.I. Inhibition of bone morphogenetic proteins protects against atherosclerosis and vascular calcification. Circ. Res. 2010, 107, $485-494$.

119. Boström, K.I.; Rajamannan, N.M.; Towler, D.A. The regulation of valvular and vascular sclerosis by osteogenic morphogens. Circ. Res. 2011, 109, 564-577.

120. O’Young, J.; Liao, Y.; Xiao, Y.; Jalkanen, J.; Lajoie, G.; Karttunen, M.; Goldberg, H.A.; Hunter, G.K. Matrix Gla protein inhibits ectopic calcification by a direct interaction with hydroxyapatite crystals. J. Am. Chem. Soc. 2011, $133,18406-18412$.

121. Schurgers,L.J.;Spronk,H.M.H.;Skepper,J.N.;Hackeng,T.M.;Shanahan,C.M.;Vermeer,C.;Weissberg,P.L.; Proudfoot, D. Post-translational modifications regulate matrix Gla protein function: Importance for inhibition of vascular smooth muscle cell calcification. J. Thromb. Haemost. 2007, 5, 2503-2511.

122. Khavandgar, Z.; Roman, H.; Li, J.; Lee, S.; Vali, H.; Brinckmann, J.; Davis, E.C.; Murshed, M. Elastin haploinsufficiency impedes the progression of arterial calcification in MGP-deficient mice. J. Bone Miner. Res. 2014, 29, 327-337.

123. Price, P.A.; Toroian, D.; Lim, J.E. Mineralization by inhibitor exclusion: The calcification of collagen with fetuin. J. Biol. Chem. 2009, 284, 17092-17101.

124. Cancela, M.L.; Conceição, N.; Laizé, V. Gla-rich protein, a new player in tissue calcification? Adv. Nutr. 2012, 3 , $174-181$. 
125. Viegas, C.S.B.; Rafael, M.S.; Enriquez, J.L.; Teixeira, A.; Vitorino, R.; Luís, I.M.; Costa, R.M.; Santos, S.; Cavaco, S.; Neves, J.; et al. Gla-rich protein acts as a calcification inhibitor in the human cardiovascular system. Arterioscler. Thromb. Vasc. Biol. 2015, 35, 399-408.

126. Eitzinger,N.;Surmann-Schmitt,C.;Bösl,M.;Schett,G.;Engelke,K.;Hess,A.;vonderMark,K.;Stock,M. Ucma is not necessary for normal development of the mouse skeleton. Bone 2012, 50, 670-680.

127. Geleijnse,J.M.;Vermeer,C.;Grobbee,D.E.;Schurgers,L.J.;Knapen,M.H.J.;vanderMeer,I.M.;Hofman,A.; Witteman, J.C.M. Dietary intake of menaquinone is associated with a reduced risk of coronary heart disease: The Rotterdam Study. J. Nutr. 2004, 134, 3100-3105.

128. Juanola-Falgarona,M.;Salas-Salvadó,J.;Martínez-González,M.Á.;Corella,D.;Estruch,R.;Ros,E.;Fitó,M.; Arós, F.; Gómez-Gracia, E.; Fiol, M.; et al. Dietary intake of vitamin $\mathrm{k}$ is inversely associated with mortality risk. J. Nutr. 2014, 144, 743-750.

129. Beulens, J.W.; Bots, M.L.; Atsma, F.; Bartelink, M.L.E.; Prokop, M.; Geleijnse, J.M.; Van Der Schouw, Y.T. High dietary menaquinone intake is associated with reduced coronary calcification. Atherosclerosis 2009, $203,489-493$.

130. Westenfeld, R.; Krueger, T.; Schlieper, G.; Cranenburg, E.C.M.; Magdeleyns, E.J.; Heidenreich, S.; Holzmann, S.; Vermeer, C.; Jahnen-Dechent, W.; Ketteler, M.; et al. Effect of vitamin $\mathrm{K}_{2}$ supplementation on functional vitamin K deficiency in hemodialysis patients: A randomized trial. Am. J. Kidney Dis. 2012, 59, 186-195.

131. Theuwissen, E.; Teunissen, K.J.; Spronk, H.M.H.; Hamulyak, K.; ten Cate, H.; Shearer, M.J.; Vermeer, C.; Schurgers, L.J. Effect of low-dose supplements of menaquinone-7 (vitamin $\mathrm{K}_{2}$ ) on the stability of oral anticoagulant treatment: Dose-response relationship in healthy volunteers. J. Thromb. Haemost. 2013, 11, 1085-1092.

132. Buitenhuis, H.C.; Soute, B.A.; Vermeer, C. Comparison of the vitamins K1, K2 and K3 as cofactors for the hepatic vitamin K-dependent carboxylase. Biochim. Biophys. Acta 1990, 1034, 170-175.

133. Schlieper,G.;Westenfeld,R.;Krüger,T.;Cranenburg,E.C.;Magdeleyns,E.J.;Brandenburg,V.M.;Djuric,Z.; Damjanovic, T.; Ketteler, M.; Vermeer, C.; et al. Circulating nonphosphorylated carboxylated matrix Gla protein predicts survival in ESRD. J. Am. Soc. Nephrol. 2011, 22, 387-395.

134. Ueland, T.; Dahl, C.P.; Gullestad, L.; Aakhus, S.; Broch, K.; Skårdal, R.; Vermeer, C.; Aukrust, P.; Schurgers, L.J. Circulating levels of non-phosphorylated undercarboxylated matrix Gla protein are associated with disease severity in patients with chronic heart failure. Clin. Sci. 2011, 121, 119-127.

135. Cranenburg,E.C.M.;Vermeer,C.;Koos,R.;Boumans,M.-L.;Hackeng,T.M.;Bouwman,F.G.;Kwaijtaal,M.; Brandenburg, V.M.; Ketteler, M.; Schurgers, L.J. The circulating inactive form of matrix Gla Protein (ucMGP) as a biomarker for cardiovascular calcification. J. Vasc. Res. 2008, 45, 427-436.

136. Brandenburg, V.M.; Schurgers, L.J.; Kaesler, N.; Püsche, K.; van Gorp, R.H.; Leftheriotis, G.; Reinartz, S.; Koos, R.; Krüger, T. Prevention of vasculopathy by vitamin K supplementation: Can we turn fiction into fact? Atherosclerosis 2015, 240, 10-16.

137. Morishima, Y.; Kamisato, C.; Honda, Y.; Furugohri, T.; Shibano, T. The effects of warfarin and edoxaban, an oral direct factor Xa inhibitor, on $\gamma$ carboxylated (Gla-osteocalcin) and undercarboxylated osteocalcin (uc-osteocalcin) in rats. Thromb. Res. 2013, 131, 59-63.

138. Krüger, T.; Oelenberg, S.; Kaesler, N.; Schurgers, L.J.; van de Sandt, A.M.; Boor, P.; Schlieper, G.; Brandenburg, V.M.; Fekete, B.C.; Veulemans, V.; et al. Warfarin induces cardiovascular damage in mice. Arterioscler. Thromb. Vasc. Biol. 2013, 33, 2618-2624. 
ANTICOAGULATION AND THE VASCULATURE 


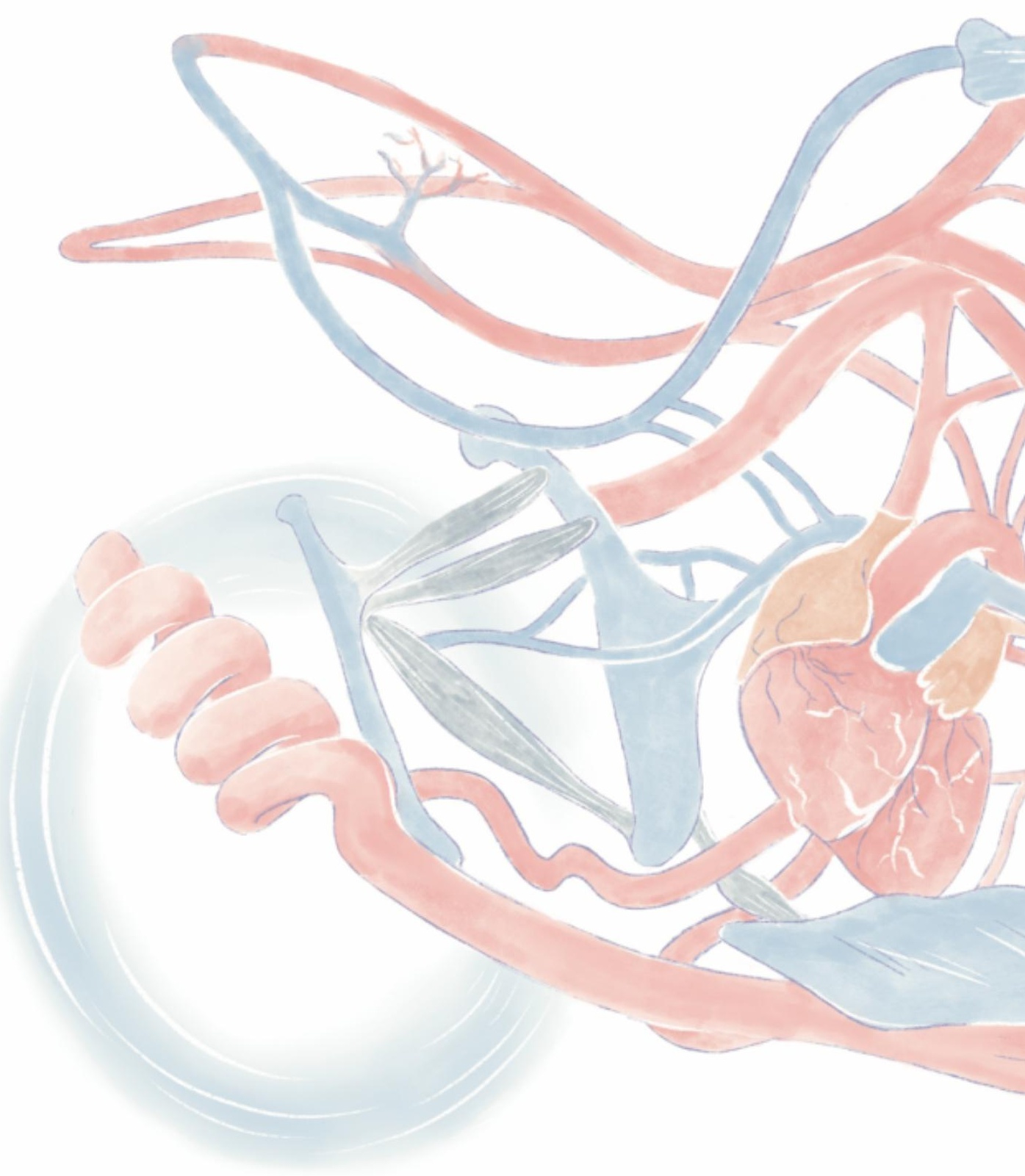




\section{Chapter 3}

Vitamin $K$ antagonist induces calcification and atherosclerotic plaque progression resulting in increased hypercoagulability

Rick H. van Gorp, Constance C.F.M.J. Baaten, Frederique E.C.M. Peeters, Peter Leenders, Harry Crijns, Johan Heemskerk, Chris P. Reutelingsperger, Henri M. Spronk, Leon J. Schurgers 


\title{
Chapter 3
}

\begin{abstract}
Aims. Vascular calcification is a hallmark of atherosclerotic burden and can predict cardiovascular outcome. Vitamin K-antagonists (VKA) are widely used anticoagulant drugs to treat patients at risk of arterial and venous thrombosis, but are also known to increase vascular calcification progression. Here we aim to unravel the paradox that VKA suppress plasma coagulation but promote atherosclerosis-dependent coagulability of the vessel wall. Methods and results. Apoe $e^{-/}$mice on western type diet enriched with the VKA warfarin showed increases in both atherosclerotic plaque progression and calcification. Higher plaque calcification was associated with increased plasma levels of thrombin-antithrombin and factor IXa-antithrombin complexes in mice and patients treated with VKA. Mechanistically, phenotypic switching of vascular smooth muscle cells (VSMC) into synthetic VSMC promotes thrombin generation, which is enhanced in a tissue-factor (TF)-dependent manner by VSMC calcification. Moreover, calcified VSMC exposed to whole blood under flow significantly enhanced platelet deposition and TF-dependent fibrin formation.
\end{abstract}

Conclusions. Oral anticoagulation with VKA aggravates vascular calcification and atherosclerosis. VSMC phenotype differentiation influences coagulation potential in a TFdependent manner. Vascular calcification increases hypercoagulability and could thereby potentially positively affect atherothrombosis. 


\section{Introduction}

Vascular calcification is clinically assessed by computed tomography (CT), and is currently used as independent predictor of cardiovascular burden, event and mortality (1). Patients displaying vascular calcification are four times more likely to suffer from cardiovascular events in a time span of 3 to 5 years (2). Markedly, also an annual increase in vascular calcification has been found to be predictive for the risk of cardiovascular disease (3). Patients who experience a yearly $>15 \%$ increases in vascular calcification are considered to have a 17 fold increased risk of myocardial infarction, when compared to patients without significant vascular calcification-progression (4).

Despite the strong association between vascular calcification progression and cardiovascular outcomes, the mechanism of how calcification alters the atherosclerotic plaque is unclear and subject to debate. CT quantifies calcification volume and density, but because of the poor resolution only reflects macrocalcifications. CT is not able to detect microcalcifications, nor metabolic activity of calcification $(5,6)$. The presence of microcalcifications, also termed spotty calcification, is strongly associated with plaque progression and vulnerability $(7,8)$. While microcalcification is a key feature of high risk atheroma plaques, recent studies put forward that macrocalcification confers plaque stability $(5,9)$. The rationale for this comes from studies with intensive lipid-lowering therapy, showing reduces risk of cardiovascular events and at the same time accelerated progression of vascular calcification (7). Moreover, plaque volume correlates with increased cardiovascular events, whereas an inverse association has been reported between coronary artery calcification (CAC) density and cardiovascular events (8).

Atherothrombosis is characterized by plaque rupture or progressive stenotic narrowing of the lumen to such an extent that platelet thrombi can occlude the vessel lumen completely. Such occlusive thrombi in coronary arteries would arrest flow and cause ST-segment elevation myocardial infarction (9). Acute coronary syndromes without ST-segment elevation results from an incomplete or transient obstruction of flow in the culprit coronary artery at a site of critical stenosis.

Vitamin K-antagonist (VKA) treatment is used to reduce risk of recurrent thrombosis but as a side effect accelerates vascular calcification (10). Recently, VKA treatment was shown to be associated with increased arterial stiffness, higher LV mass, and decreased cardiac systolic function (11). Experiments in rodents have shown that VKA treatment shifts atherosclerotic plaques towards a vulnerable phenotype by inducing both medial and intimal calcification, increasing apoptosis and promoting inward remodeling (12). On the other hand, accelerated thrombin formation is predictive for presence and severity of CT angiographic coronary atherosclerosis (13).

In this study we aim to unravel the apparent paradox that VKA lower coagulation tendency and induce atherosclerotic plaque progression and calcification yet increase hypercoagulability. 


\section{Materials and methods}

Experimental animals. Animal studies were performed under an approved protocol by the animal ethics committee of Maastricht University. Twelve-week-old female C57/BL6 Apoe 1- mice were housed in climate-controlled cages under 12-hour day/night cycle with ad libitum access to food and water. Mice were fed an irradiated (0.9 Mrad) vitamin K-deficient Western type diet (WTD: $0.25 \%$ cholesterol and 15\% cocoa butter, derived from Altromin, Lage, Germany). For the control group, the WTD was supplemented with vitamin K1 (5 $\mathrm{mg} / \mathrm{Kg}$, Merck KGaA, Darmstadt, Germany) dissolved in corn oil. For the experimental group, this diet was supplemented with warfarin and vitamin $\mathrm{K} 1$ (3 mg/g warfarin $+1.5 \mathrm{mg} / \mathrm{g}$ vitamin K1, Merck KGaA, Darmstadt, Germany). Vitamin K1, counteracting warfarin effects in the liver was added in addition to warfarin in order to prevent bleeding, but allowing vitamin K-deficiency in the vasculature, such as described previously $(15,17)$. Mice were randomly assigned to receive control or warfarin diet for 18 weeks.

After the treatment period of 18 weeks, mice were sacrificed, and blood was collected from the portal vein into $105 \mathrm{mM}$ trisodium citrate. Plasma was prepared and aliquots were frozen at $-80^{\circ} \mathrm{C}$ until analysis. After bleeding, the mouse vasculature was washed by injection of a sterile isotonic buffer ( $40 \mathrm{mM}$ Hepes, $150 \mathrm{mM} \mathrm{NaCl}, 5 \mathrm{mM} \mathrm{KCl}, 1 \mathrm{mM} \mathrm{MgCl}_{2}$ and $2.5 \mathrm{mM}$ $\mathrm{CaCl}_{2}, \mathrm{pH} 7.3$ ) via the left ventricle. Aortas were harvested, dissected and fixed overnight into $1 \%(\mathrm{v} / \mathrm{v})$ Hepes-buffered paraformaldehyde, containing $150 \mathrm{mM}$ saline at $4^{\circ} \mathrm{C}$, before embedding into paraffin.

Study population. In a cross-sectional observational study, we included 54 patients displaying paroxysmal atrial fibrillation (AF) with a low cardiovascular risk, who were treated with VKA (acenocoumarol). The patient cohort has been described previously (15, 16). The patients were diagnosed of paroxysmal AF by cardiac multislice computed tomography (MSCT). Exclusion criteria were symptoms of untreated cardiovascular disease, hypertension, diabetes mellitus, hypercholesteremia, coronary artery disease, chronic kidney disease or heart failure. For each patient, Agatston score was calculated from CT scans, using a $3 \mathrm{~mm}$ slice thickness and a detection threshold of $\geq 130 \mathrm{HU}$ involving $\geq 1 \mathrm{~mm}^{2}$ area/lesion (3 pixels). After scanning, blood was drawn by venipuncture into $3.2 \%$ trisodium citrate. Plasma samples were prepared and stored at $-80{ }^{\circ} \mathrm{C}$ until analysis.

Blood analysis. Warfarin levels in plasma were determined using HPLC. Plasma levels of total cholesterol and triglycerides were assessed using a Synchron LX20 (Beckman Coulter, Brea, USA). Factor IXa-antithrombin (FIXa:AT) and thrombin-antithrombin (TAT) complex levels were determined via in house developed assays, as described previously (17).

Immunohistochemistry. Mouse aortic sections embedded in parafin were cut at $4 \mu \mathrm{m}$ thickness. In selected sections, plaque size was determined by hematoxylin and eosin staining. In adjacent sections, plaque calcification was determined by staining with Alizarin Red S. Stained sections were analyzed by ImageJ Software. Other sections were immunostained with antibody against anti-uncarboxylated matrix Gla-protein (1:400; IDS, 
Boldon, UK), in order to assess for vascular vitamin K deficiency (18). Visualization was with a HRP conjugated secondary antibody and Nova-RED substrate (Vector Labs, Amsterdam, the Netherlands). Two-dimensional image analysis was performed using a Leica Application Suite X microscope and software (Leica Microsystems, Wetzlar, Germany). Data were expressed as percentage coverage of plaque area, or as stated otherwise.

Cell culture. Human primary vascular smooth muscle cells (VSMC) were derived from tissue explants from males and females aged between 18 and 65 years of age. The cells were cultured, as described (19). In brief, the VSMC were hold in DMEM medium (Gibco, Thermo Fisher Scientific, Paisley, Scotland, UK) supplemented with $20 \%$ fetal bovine serum (FBS), $100 \mathrm{U} / \mathrm{mL}$ penicillin and $100 \mu \mathrm{g} / \mathrm{mL}$ streptomycin. Cells were used between passages 5 and 10 .

Flow cytometry. Matrix Gla protein (MGP) carboxylation status of VSMC was determined after fixing the cells in 4\% paraformaldehyde. Hereafter, the cells were stained with primary antibody against uncarboxylated MGP (ucMGP) or against carboxylated MGP (cMGP, 1:400; IDS, Boldon, UK), followed by a secondary FITC-labeled anti-mouse IgG (1:250, Hycult Biotechnology, Wayne, USA). MGP intensity was measured using flow cytometry.

Tissue factor activity. Tissue factor (TF) activity was determined in lysates of VSMC, as previously described (20).

Calcification assays. VSMC were placed in calcifying medium consisting of DMEM supplemented with $2.5 \% \mathrm{FBS}, 100 \mathrm{U} / \mathrm{mL}$ penicillin, and $100 \mu \mathrm{g} / \mathrm{mL}$ streptomycin in the presence of additional $1.8 \mathrm{mmol} / \mathrm{L} \mathrm{CaCl}_{2}$. After growing and incubation with warfarin (10$100 \mu \mathrm{M}$ ), calcium deposits were assessed by the O-cresolphthalein method. Amounts of calcium deposits were normalized for protein content (micro BSA protein kit, Thermo Fisher Scientific, Paisley, UK).

Thrombin generation. VSMC were cultured under low serum conditions $(0.5 \% \mathrm{FBS})$ to obtain contractile VSMC. Contractile VSMC were plated in 12 wells until $80 \%$ confluence or incubated with PDGF (20 ng/mL, Merck GBaA, Darmstadt, Germany) to obtain synthetic VSMC, as described before (21). Synthetic cells remained in PDGF medium supplemented with or without $3.6 \mathrm{mmol} / \mathrm{L}$ additional $\mathrm{CaCl}_{2}$. After 48 hours, all types of cells were washed twice in $\mathrm{HN}$ buffer (Hepes buffered saline, $\mathrm{pH}$ 7.7) before collection in $\mathrm{HN}$ buffer containing $0.5 \%$ BSA. Cell lysates were stored at $-20{ }^{\circ} \mathrm{C}$ until analysis. Continuous thrombin generation was monitored in vitro by using the Calibrated Automated Thrombography (CAT) method (Thrombinoscope B.V., Maastricht, the Netherlands) (22). The reaction was triggered by cell lysate in the presence of either normal, factor XII- or factor VII-deficient plasma, lipids (4 $\mu \mathrm{M}$ micro phosphatidylserine/phosphatidylcholine (PS/PC) vesicles) and fluorescent substrate plus calcium (FluCa). Active-site inhibited factor VIIa (ASIS; $30 \mathrm{nM}$ ) or corn trypsin inhibitor (CTI; $25 \mu \mathrm{g} / \mathrm{mL}$ ) was added to block the intrinsic or extrinsic coagulation pathway. 
Whole-blood microfluidics assay. For microfluidics tests, VSMC were cultured on glass cover slips in cell culture dishes. Prior to the flow experiments, VSMC were differentiated with PDGF $(20 \mathrm{ng} / \mathrm{mL})$ and subsequently incubated in the presence or absence of $3.6 \mathrm{mmol} / \mathrm{L}$ $\mathrm{CaCl}_{2}$. The glass cover slips with VSMC were placed in a Maastricht flow chamber, and perfused with recalcified human whole blood containing Alexa Fluor-647 fibrinogen for 7 min at arterial wall-shear rate of $1000 \mathrm{~s}^{1}(23,24)$. After $7 \mathrm{~min}$ of flow, the cells were postperfused with rinse buffer to reveal staining of fluorescent fibrin (25). Citrated blood samples from healthy subjects were used, supplemented with the contact activation inhibitor CTI or ASIS, added after blood collection. Images were analyzed using Fiji/Image J (26).

Statistical analyses. In vivo data are presented as median with interquartile range. All in vitro data were obtained in three or more independent experiments in triplicates or more wells and data are expressed as mean with SD. Data were analyzed using Mann-Whitney Ttest or Kruskal-Wallis One-way ANOVA as indicated using PRISM software (GraphPad 8.3.0, San Diego, US). Values of $\mathrm{p}<0.05$ were considered statistically significant.

\section{Results}

Apoe $^{-/-}$mice were fed for 18 weeks as control with Western-type diet, or supplemented with warfarin, in order to determine the effect of warfarin on plaque progression and calcification (Supplemental Figure 1A). After 18 weeks, plasma triglycerides and cholesterol as well as animal body weights were similar between the two groups (Supplemental Table 1). Warfarin treated mice showed high plasma levels of warfarin, in contrast to the control mice (Supplemental Table 1), thus confirming adequate uptake of warfarin from the diet.

Supplemental Table 1. Model validation

\begin{tabular}{|c|c|c|}
\hline & Control & Warfarin \\
\hline Weight (gram) & $23.8 \pm 1.6$ & $22.5 \pm 1.7$ \\
\hline Cholesterol (mM) & $21.27 \pm 3.34$ & $21.54 \pm 3.07$ \\
\hline Triglycerides (mM) & $2.77 \pm 0.20$ & $2.96 \pm 0.28$ \\
\hline Warfarin $(\mathbf{m g} / \mathbf{L})$ & 0 & $11.52 \pm 5.81$ \\
\hline
\end{tabular}

After 18 weeks of diet, the atherosclerotic plaques from mice treated with warfarin showed significantly increased vascular calcification, as compared to the control group (Figure 1A). In addition, warfarin treatment resulted in significant increased calcification of aortic valves (supplemental Figure 1B). Furthermore, warfarin diet for 18 weeks caused an enlarged atherosclerotic plaque size, compared to control diet $\left(0.4 \pm 0.16 \mathrm{~mm}^{2}\right.$ vs. $0.54 \pm$ $0.21 \mathrm{~mm}^{2} ; \mathrm{p}<0.05$, Figure 1B). Since vascular calcification is clinically used as a marker of atherosclerotic burden, we also determined plaque progression. This parameter was quantified as fold increase in plaque size between 6 and 18 weeks of treatment; quantification 

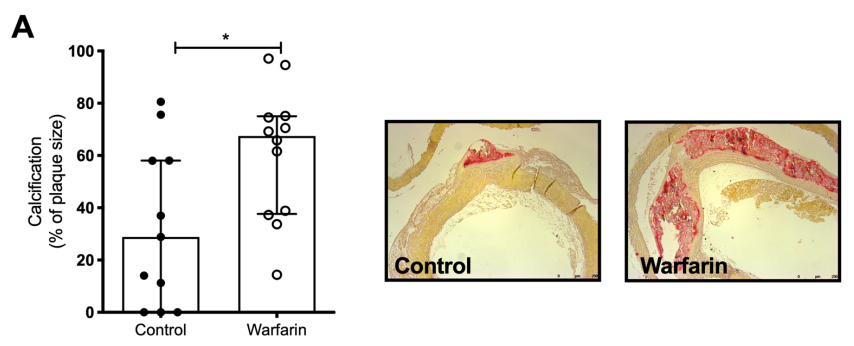

\section{C}
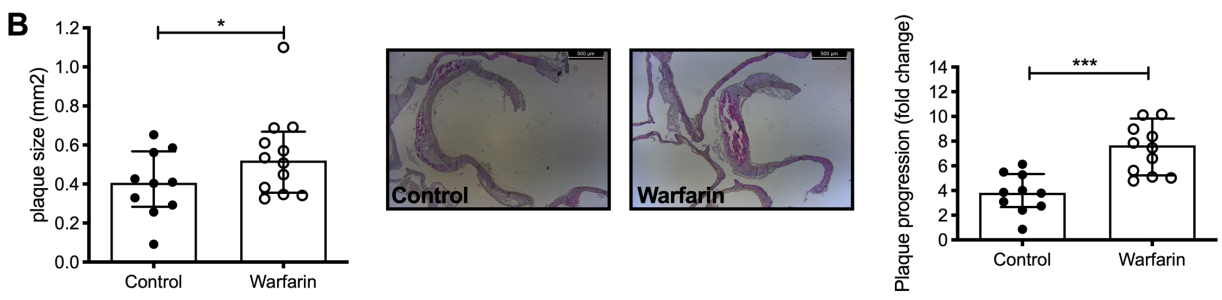

Figure 1. Warfarin treatment increases plaque calcification. A) 18 weeks of treatment demonstrated that warfarin increased calcification compared to control treatment detected by Alizarin Red S staining. B) Plaque size was significant increased after 18 weeks of warfarin treatment compared to control treatment. C) Plaque progression, determined as fold increase of plaque size from 6 weeks towards 18 weeks of treatment, was significantly increased after warfarin compared to control.

revealed that warfarin treatment significantly increased plaque progression, when compared to the control diet (3.8-fold vs. 7.6-fold; $\mathrm{p}<0.001$, Figure 1C).

Warfarin is known to inhibit the enzyme vitamin K-epoxide reductase (VKOR), thereby interfering with the recycling of vitamin $\mathrm{K}$ resulting in the accumulation of uncarboxylated proteins (27). To verify this, we quantified uncarboxylated matrix Gla protein (ucMGP) in aortic atherosclerotic plaques (12). The ucMGP expression was significantly higher in the warfarin group (Supplemental Figure 1E). In addition, levels of ucMGP strongly correlated with extent of plaque calcification $(\mathrm{r}=0.82, \mathrm{p}<0.01$; Supplemental Figure 1F).

Using cultured VSMC, we tested whether warfarin addition ( $10 \mu \mathrm{M}$ for 48 or 72 hours) also could affect MGP carboxylation in vitro. Indeed, in cultured VSMC warfarin caused a time-dependent increase in ucMGP at the expense of carboxylated MGP (cMGP) (Supplemental Figure 1C). Additionally, warfarin increased calcification of VSMC in a dosedependent way (Supplemental Figure 1D).

In patients with coronary artery disease, the severity of atherosclerosis, as measured by computed tomographic angiography, has been linked to accelerated thrombin generation (13). To evaluate this in the present setting, we determined the levels of thrombinantithrombin (TAT) and factor IXa:antithrombin (FIXa:AT) complexes in plasma from control and warfarin treated animals. 
A

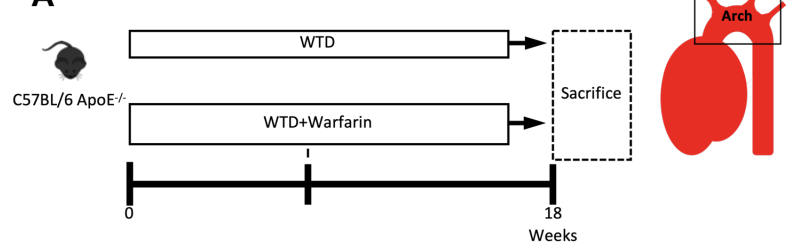

B

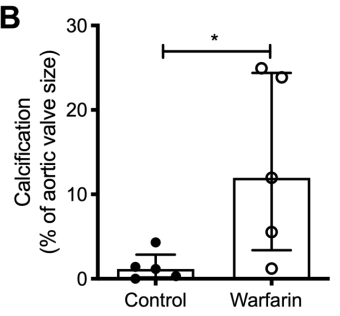

C
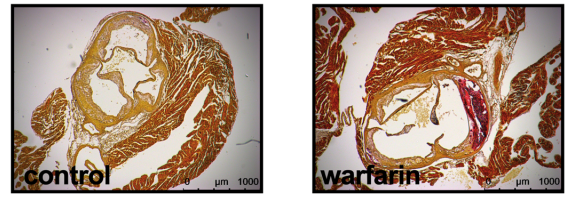

D
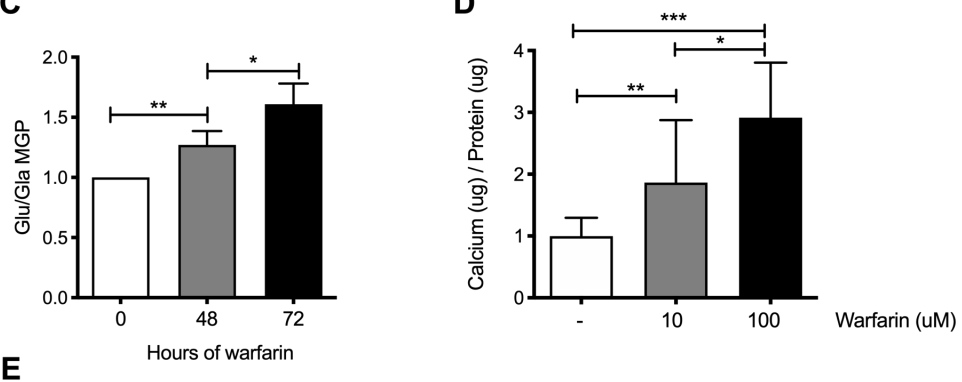

E

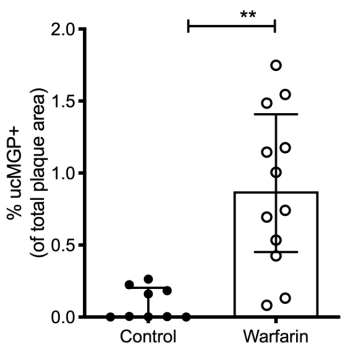

$\mathbf{F}$
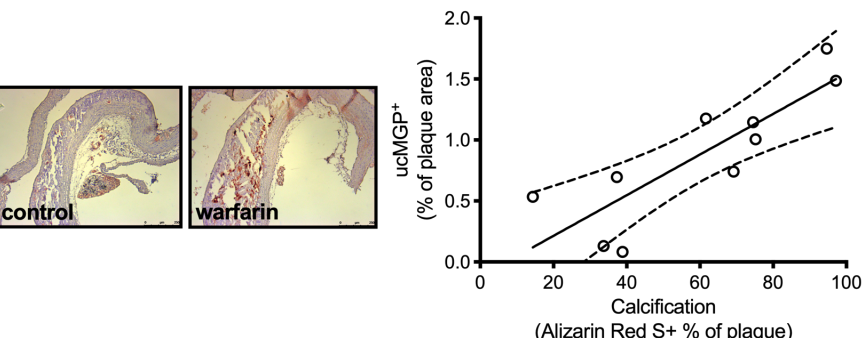

Supplemental Figure 1. Warfarin induced atherogenesis and vascular calcification is sex independent. A) Schematic overview of our experimental animal model. B) Warfarin treatment increased valve calcification after 18 weeks of treatment as compared to control. C) Warfarin $(10 \mu \mathrm{M})$ induced a time-dependent switch in MGP carboxylation status towards an uncarboxylated (Glu residue) state. D) Warfarin treatment increased calcification in VSMC in a dose dependent manner. E) Uncarboxylated MGP (ucMGP) was increased in atherosclerotic plaques after 18 weeks of warfarin treatment compared to control treatment. F) ucMGP expression in the atherosclerotic plaque correlates $(\mathrm{r}=0.82, \mathrm{p}=<0.01)$ with calcification detected by alizarin Red S. 

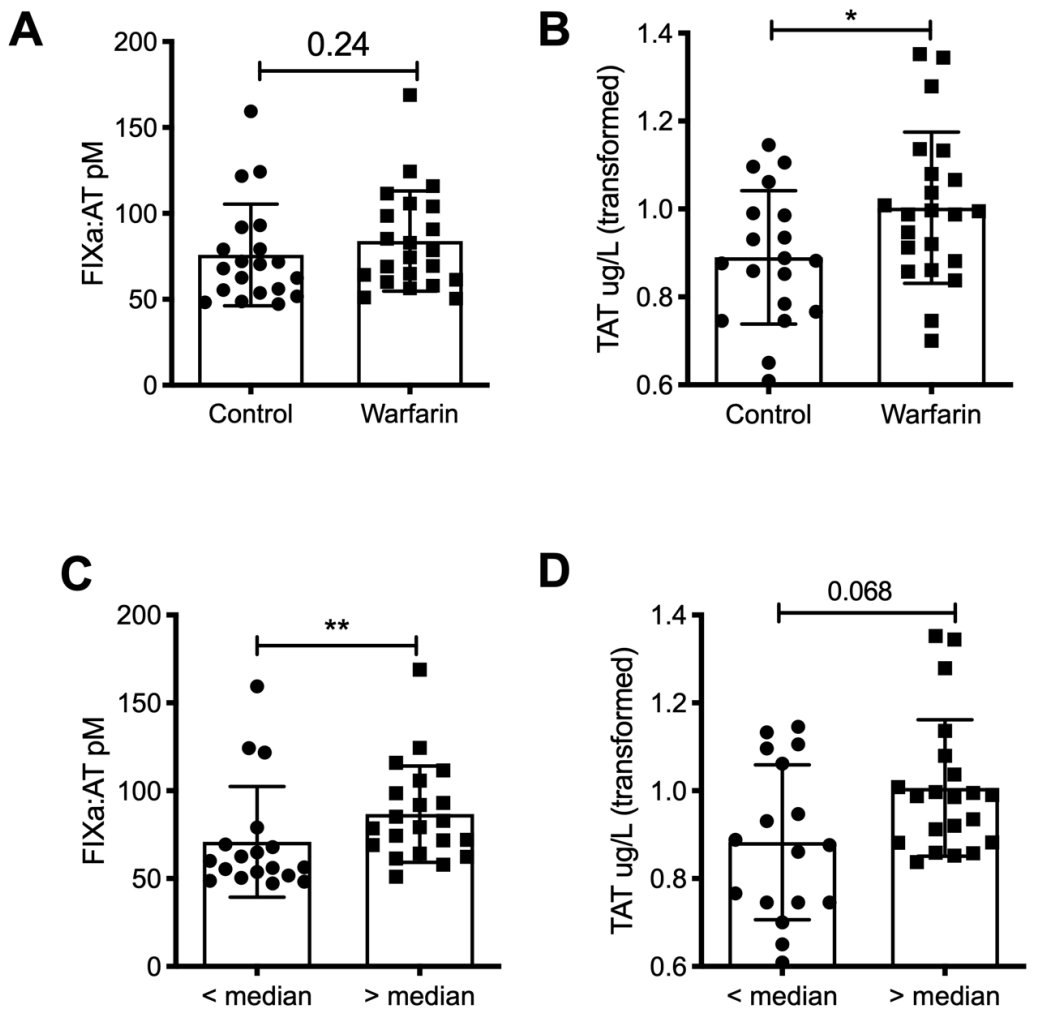

Figure 2. Vascular calcification induces a prethrombotic state. A) Circulating Factor IXa-antithrombin (FIXa:AT) levels remained similar between warfarin and control treated mice after 18 weeks. B) Warfarin treated mice had significantly increased circulating thrombin-antithrombin (TAT) levels as compared to control mice. C) Stratification of all mice by calcification revealed a significant increase in circulating FIXa:AT ratio levels in mice with higher than the median calcification percentage. D) Stratification resulted in a borderline significant increase (p 0.068) of circulating TAT levels in mice with higher than median calcification percentage.

Elevation in these complexes points to the presence of a prethrombotic state (17). Interestingly, levels of TAT but not of FIXa:AT complexes were markedly increased in mice treated with warfarin (Figure 2A, B). To assess a possible relation to extent of plaque calcification, we stratified control and warfarin treated mice based on median plaque calcification percentage (median; 58\% calcification of plaques). We noted a significant increase in FIXa:AT levels $(\mathrm{p}<0.01)$ and a close to significant increase in TAT levels $(\mathrm{p}=$ 0.068 ) in animals with calcification exceeding the median (Figures $2 \mathrm{C}$ and $\mathrm{D}$ ).

Phenotypic switching of VSMC is known to play a crucial role in maintaining structural integrity of the blood vessel (28), and in vascular calcification (29). We used lysates of contractile, synthetic or calcified VSMC to investigate consequence of VSMC phenotypic switching on coagulation stimulation in normal plasma, by measuring thrombin generation parameters using the CAT assay. Typically, synthetic VSMC showed a shorter 
A
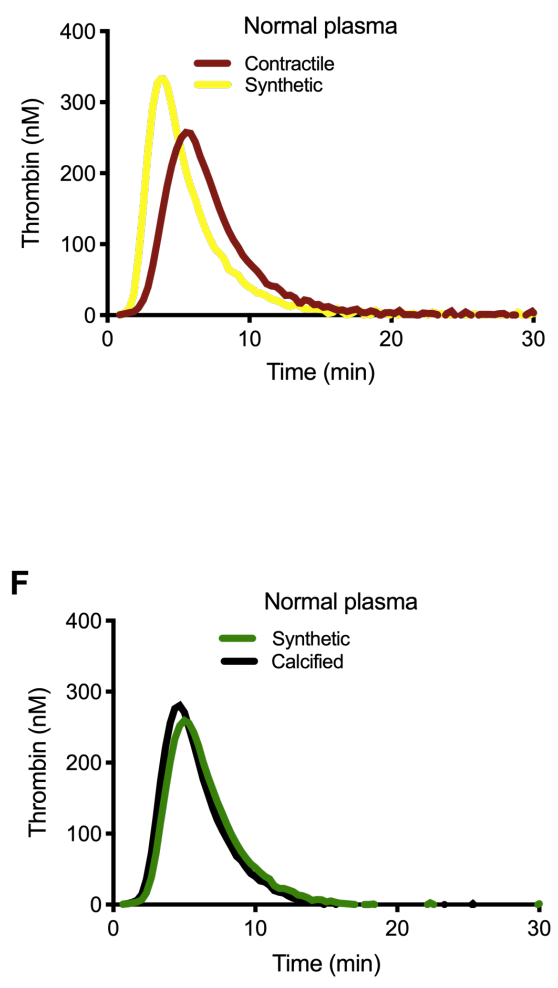

B

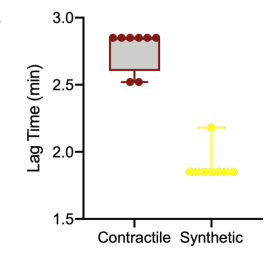

D

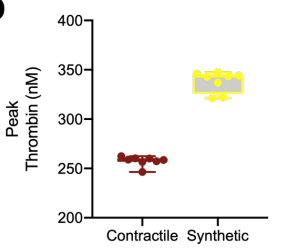

G

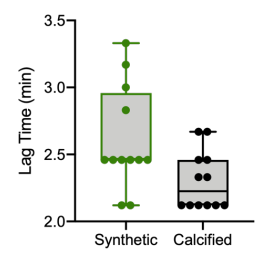

I

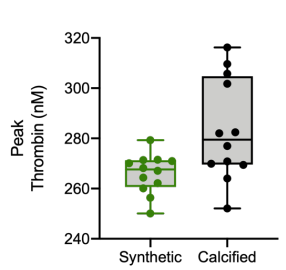

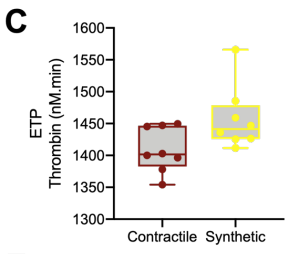

E
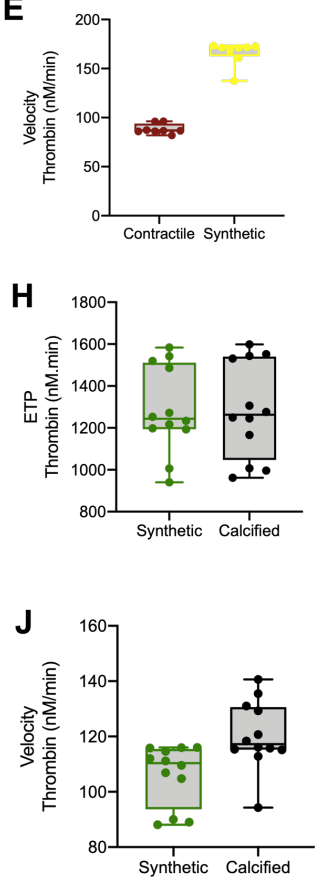

Figure 3. Thrombogenicity of vascular smooth muscle cell phenotype. A) Using normal plasma, both synthetic (yellow) and contractile (red) VSMC were inducers of thrombin generation. Synthetic VSMC showed a shorter lag time (B), slighty increased ETP (C) and higher peak (D) and velocity (E)compared to contractile VSMC. F) Calcified VSMC (dark red) demonstrated a shorter lag time (G), similar ETP (H), higher peak (I) and velocity (J) as compared to synthetic VSMC (green).

lag-time and increased thrombin peak height, velocity and endogen thrombin potential (ETP) when compared to contractile VSMC (Figure 3A-E). Lysates from calcified VSMC showed a slightly shorter lag-time and higher peak height and velocity, when compared to synthetic VSMC (Figure 3F-J). In order to eliminate a role of contact activation, further experiments were performed with factor XII deficient plasma. In this case, thrombin generation parameters were unaltered. However, administration of Active-site inhibited factor VIIa (ASIS), blocking the tissue factor (TF)-dependent coagulation pathway, prolonged the lagtime and reduced the thrombin peak height, ETP and velocity in all VSMC phenotypes (Figure 4A-J). These findings were confirmed for synthetic and calcified VSMC using factor VII deficient plasma in the presence or absence of corn trypsin inhibitor (CTI; inhibitor of factor XIIa) or ASIS (Supplemental Figure 2). 
A

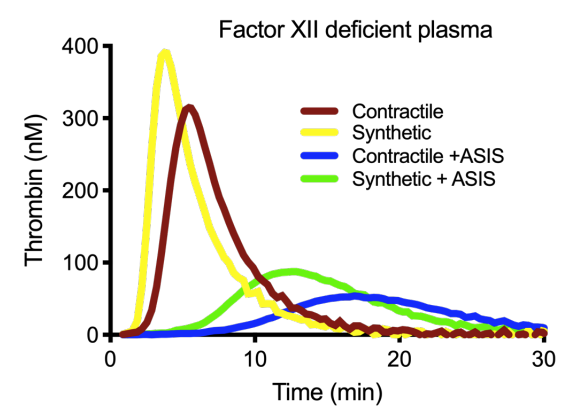

$\mathbf{F}$

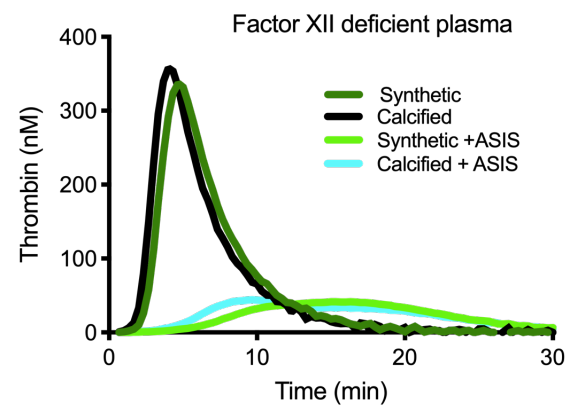

K

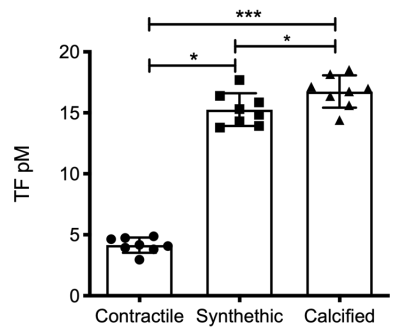

B

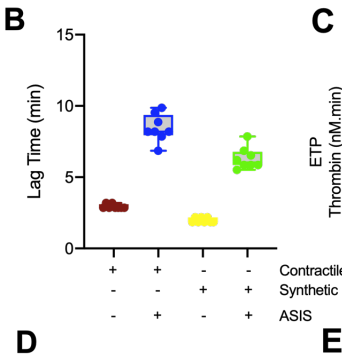

C

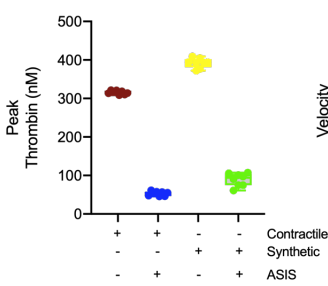

G

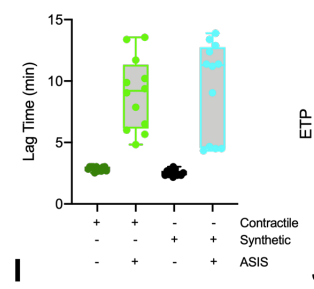

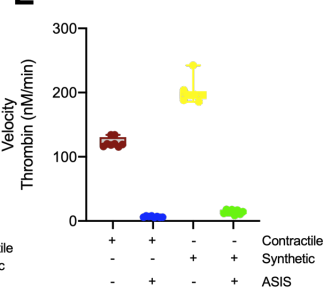

H
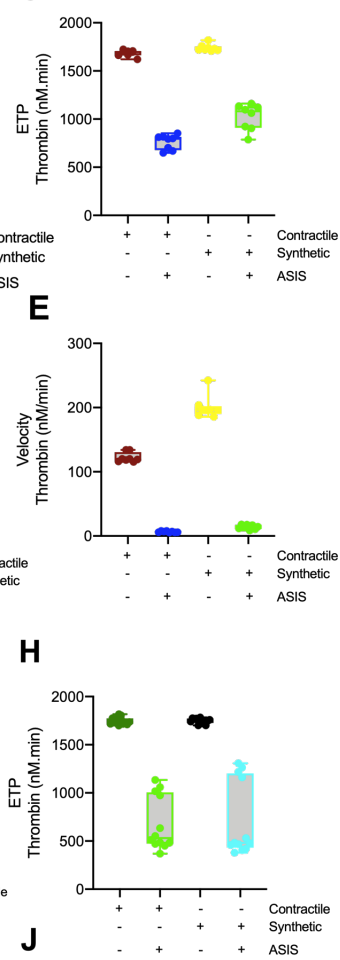
ASIS

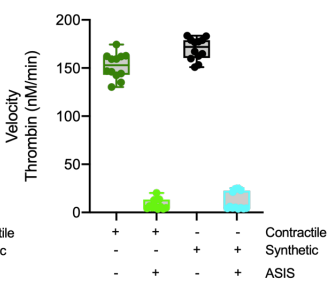

Figure 4. Intrinsic and extrinsic coagulation inhibition of vascular smooth muscle cell phenotype. Thrombin generations of contractile, synthetic and calcified VSMC were unaffected by factor XII deficient plasma compared to normal plasma (A and F). Supplementation of activated site inhibitor seven (ASIS) in factor XII deficient plasma increased lag time (B and $\mathrm{G})$ and reduced ETP $(\mathrm{C}$ and $\mathrm{H})$, peak height (D and $\mathrm{I}$ ) and velocity (E and $\mathrm{J}$ ) of all VSMC phenotypes synthetic. K) Contractile VSMC showed significant lower TF activity levels as compared to synthetic and calcified VSMC. Calcified VSMC show a significant increase in TF activity as compared to synthetic VSMC. 
A

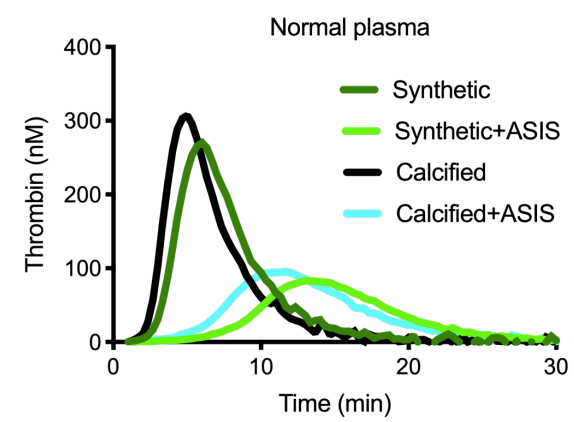

$\mathbf{F}$

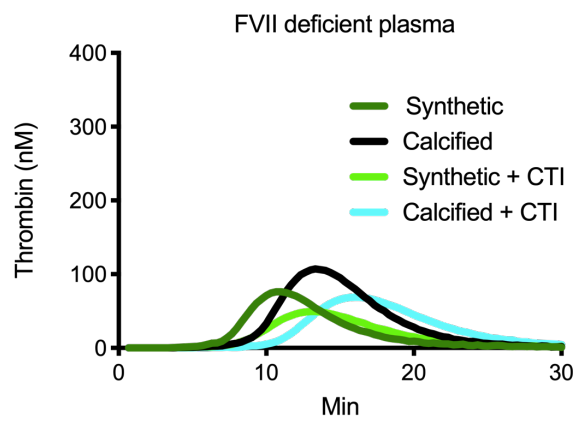

B
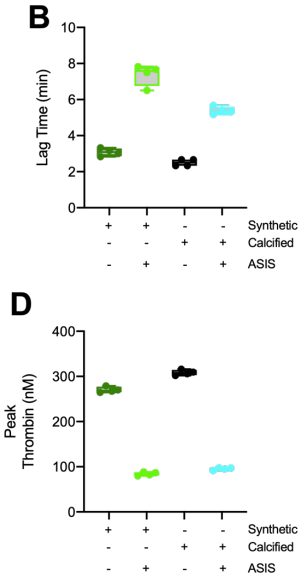

G

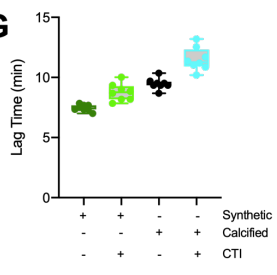

I

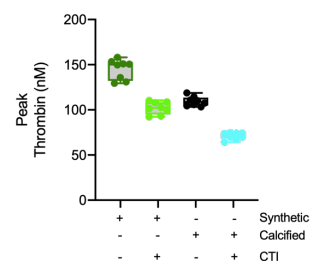

C
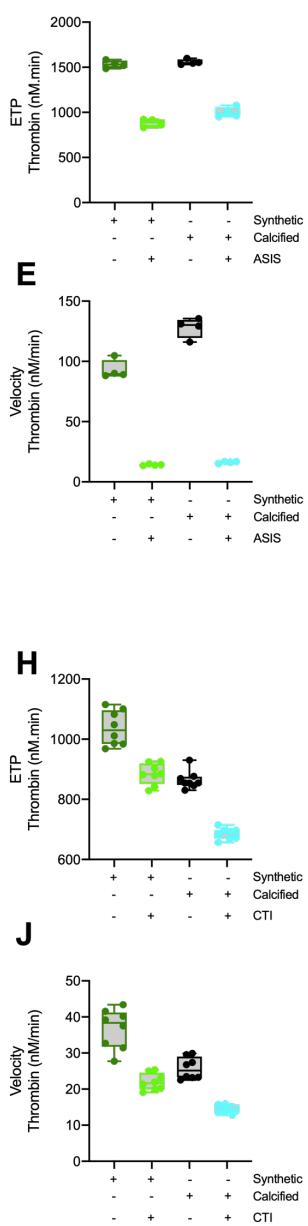

Supplemental Figure 2. Thrombin generation in VSMC is tissue factor dependent. A) In normal plasma, addition of ASIS increased lag time (B) and reduced ETP (C), peak height (D) and velocity (E) in synthetic and calcified VSMC lysates. F) Similarly, thrombin generation in FVII deficient plasma resulted in long lag times and short peak heights. Addition of CTI in FVII deficient plasma slightly increased lag times (G), reduced ETP (H), peak (I) and velocity (J) in synthetic and calcified VSMC.

Here, the use of factor VII deficient plasma or addition of ASIS reduced the thrombin peak height and prolonged the lag time. Since the extrinsic pathway seems key in VSMC-mediated activation of thrombin we measured TF procoagulant activity of VSMC lysates. TF activity was significantly increased in synthetic VSMC compared to contractile VSMC (Figure 4K). Moreover, calcified synthetic VSMC showed a further increase in TF activity, when compared to non-calcified synthetic VSMC ( $\mathrm{p}<0.05$; Figure $4 \mathrm{~K})$.

To further prove the TF-mediated procoagulant activity, we investigated the coagulation ability of calcified or non-calcified synthetic VSMC cultured in a microfluidic 
A

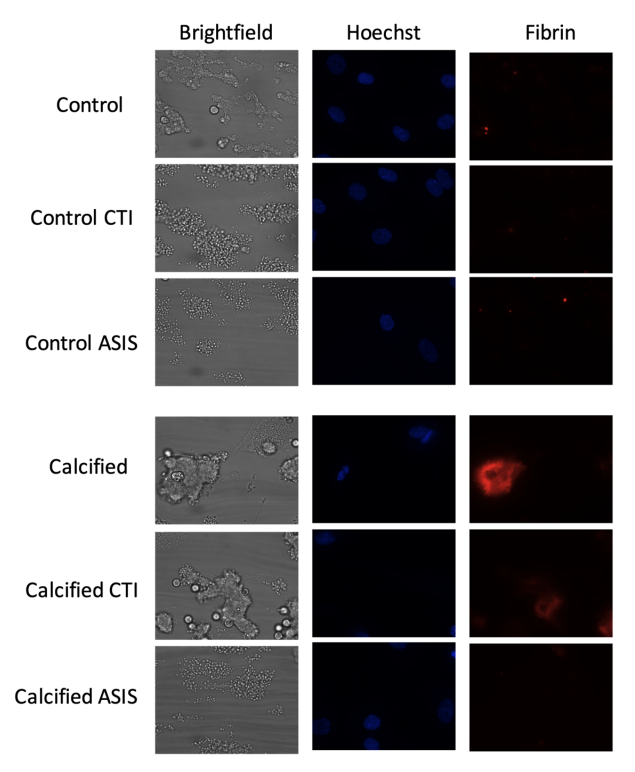

B

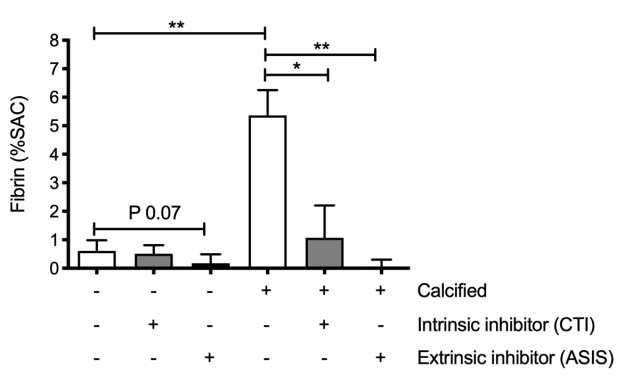

C

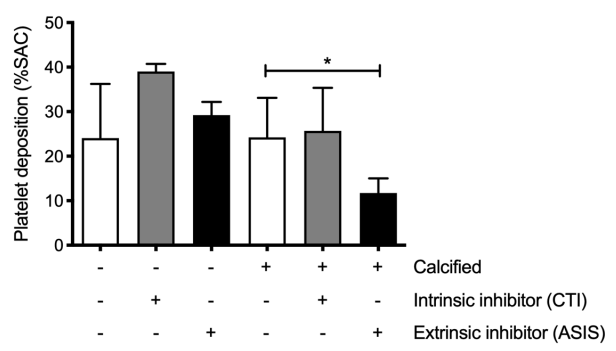

Figure 5. Thrombogenicity of vascular smooth muscle cells under flow conditions. A) Synthetic VSMC were cultured on a glass coverslip under conditions stimulating calcification or not. The cells were then mounted in a Maastricht flow chamber, and subjected to perfusion with recalficied whole blood flowed for $7 \mathrm{~min}$ at wall-shear rate of 1000/s. Shown are representative images of platelet thrombus formation (brightfield), VSMC nuclei (DAPI, blue) and AF647-fibrin formation (red). B) Quantification of fluorescent fibrin formation, indicating an increase in the presence of calcification, and a reduction in the presence of either CTI or ASIS. C) Platelet deposition on calcified VSMC was reduced in the presence of ASIS.

device, when subjected to whole blood flow. Markedly, calcified VSMC showed an increased thrombin- dependent fibrin formation and denser platelet thrombus formation Figure 5A and B). To investigate whether the fibrin formation was induced via the intrinsic or extrinsic coagulation pathway, CTI or ASIS was added to the flowed blood. Using calcified VSMC, the CTI treatment resulted in an attenuation of fibrin formation $(\mathrm{p}<0.05)$, whereas ASIS pre-treatment completely prevented fibrin formation $(\mathrm{p}<0.01)$. Moreover, ASIS significantly reduced the platelet thrombus formation (Figure 5C). Together, these data indicate that VSMC calcification enhances the cellular coagulant activity, predominantly in a TF dependent manner.

To further evaluate if patients with high vascular calcification show evidence for a prethrombotic state, we analyzed the TAT and FIXa:AT levels in VKA treated patients with or without CAC. It appeared that the presence of CAC (Agatston score $>0$ ) significantly associated with higher FIXa:AT levels, but not with TAT levels (Figure 6A and B, respectively). 
A

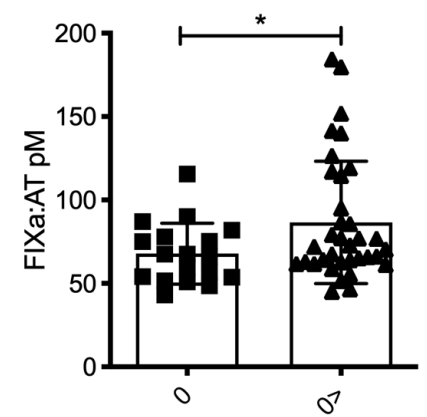

B

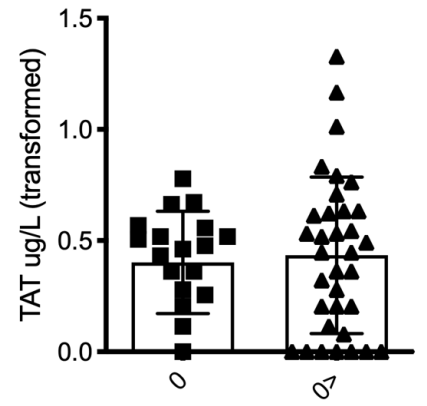

Figure 6. Vascular calcification promotes a prethrombotic state in patients on VKA. VKA treated patients with coronary calcification (Agatston score $>0$ ) compared to no coronary calcification (Agatston score 0 ) showed no difference on circulating TAT levels, (A) but significantly increased circulating FIXa:AT levels (B).

\section{Discussion}

In the present study we demonstrated that VKA treatment with warfarin increased both the progression and calcification of atherosclerotic plaques in atherosclerosis prone mice. Increased calcification was accompanied by overall increased plasma levels of TAT and FIXa:AT. We show that calcified synthetic VSMC express higher levels of TF-activity as compared to synthetic and contractile VSMCs. Furthermore, calcified VSMC caused significantly more platelet aggregation and fibrin formation when exposed to flowing whole blood as compared to non-calcified VSMC. Taken together our data indicate that calcification renders atherosclerotic lesions more prone to cause atherothrombosis.

Vascular calcification is highly prevalent in cardiovascular disease and is associated with increased cardiovascular mortality and morbidity (2). CAC strongly associates with atherosclerosis and progression of CAC is a strong predictor of future adverse cardiac events (4). VKA has been shown to increase vascular calcification $(13,30)$. Here we confirm that VKA treatment enhances atherosclerotic plaque calcification (12). Recently it has been shown that VKA treatment enhances calcification and increases plaque volume in patients with non-valvular atrial fibrillation undergoing coronary computed tomographic angiography with one-year follow up as compared to apixaban (31). It has been put forward that statin treatment is associated with a favorable cardiovascular outcome and increased vascular calcification, yet a lower plaque volume (32). Our data demonstrate that VKA treatment increases both calcification and atherosclerotic plaque size, indicating different mechanisms and impact on plaque stability (33).

Vascular calcification has long been regarded as passive, an end-stage in vascular disease resulting from chronic inflammation. During recent years, this view has changed dramatically. Vascular calcification is now appreciated to be a regulated process involving active participation of VSMC (29). VKA-induced calcification is most likely mediated via 
impaired carboxylation of vitamin K-dependent MGP and Gla Rich Protein (GRP) (18, 34). Gla residues in circulating coagulation factors, prothrombin in particular, also contribute to inhibition of vascular calcification (35). One of the strongest inhibitors of vascular calcification is MGP synthesized by VSMC (36). Increased levels of inactive MGP significantly correlates with increased vascular calcification in atrial fibrillation patients (15) and cardiovascular mortality in patients with coronary artery disease (37). Indeed, ucMGP positivity in the atherosclerotic plaque significantly correlated with amount of atherosclerotic plaque calcification, and also VSMC in vitro produced inactive ucMGP in response to VKA treatment. It is noteworthy to mention that we visualized atherosclerotic calcification using Alizarin Red S, a calcification stain that strongly correlates with the PET tracer $\mathrm{NaF}^{18}$ that is known to detect active calcification (38).

Patients with no or very low CAC scores have low risk on future clinical CVD events compared to patients with higher CAC scores (39). Moreover, CAC score is used as marker for atherosclerotic plaque burden (1). This confront us with the intriguing and crucial question as to whether calcium deposits in atherosclerotic plaques contribute to plaque stability or increase plaque instability. Upon rupture of an atherosclerotic plaque, thrombosis occurs which is termed atherothrombosis. In the arterial circulation atherothrombosis causes myocardial infarction and stroke, the most common causes of death in the Western world (40). Platelets play a pivotal role in vascular occlusive disease where they contribute to fibrin formation and inflammation $(41,42)$.

Because trace amounts of thrombin are continuously formed under physiological conditions, complexes of active serine proteases with their natural inhibitor, antithrombin, are always detectable. FIXa:AT reflects an early part of the coagulation cascade, immediately prior to factor $\mathrm{X}$ and prothrombin conversion. Additionally, TAT complex is a marker of downstream coagulation activity. Patients with increased CAC were shown to have increased FXIIa levels $(43)$ and TAT complex levels $(13,44)$. In low-risk paroxysmal atrial fibrillation patients increased FIXa:AT suggests an indication for future hypercoagulability (17). Subsequently, hypercoagulability promotes atherogenesis and atrial fibrillation $(45,46)$. We show that FIXa:AT ratio was significantly increased in presence of increased vascular calcification. Therefore, our data indicate that presence of calcification promotes a prethrombotic state. To further unravel the mechanism by which calcification activates coagulation, we used VSMC in vitro. Upon endothelial disruption, TF is released which ignites the extrinsic coagulation pathway via activation of FVII (47). VSMC normally express low levels of TF but during atherogenesis TF is expressed abundantly (48). In the atherosclerotic plaque, VSMC acquire a synthetic phenotype. Here we show that synthetic VSMC harbor significantly more TF activity compared to contractile VSMC and that calcified VSMC have even more TF activity, which is in line with increased endogenous thrombin potential (ETP).

It has been shown that membrane-associated platelet polyphosphate is condensed into insoluble spherical nanoparticles with divalent metal ions such as calcium, and that these 
calcium-phosphate particles activate factor XII (49). We showed that the initiation of coagulation was in part mediated via the intrinsic pathway but completely abolished by inhibition of the extrinsic pathway. The importance of TF activity of VSMC was shown in mice lacking VSMC derived TF, which had reduced thrombus formation after ferric chloride injury (50). It has also been shown that VSMC exposed to flow reveal a burst of TF expression (51), confirming involvement of the extrinsic coagulation pathway. Moreover, in aortic valves, TF expression was increased in calcified compared to non-calcified regions (52), suggesting that calcification correlates with TF expression. Local calcified vascular areas might initiate fibrin formation, which is known to promote atherogenesis and plaque instability (53). These data were confirmed by our in vitro flow experiments, showing that calcified VSMC caused significantly more fibrin formation. Taken together, this suggest that atherosclerotic calcified plaques harbor more TF activity, thereby driving atherothrombosis upon plaque rupture.

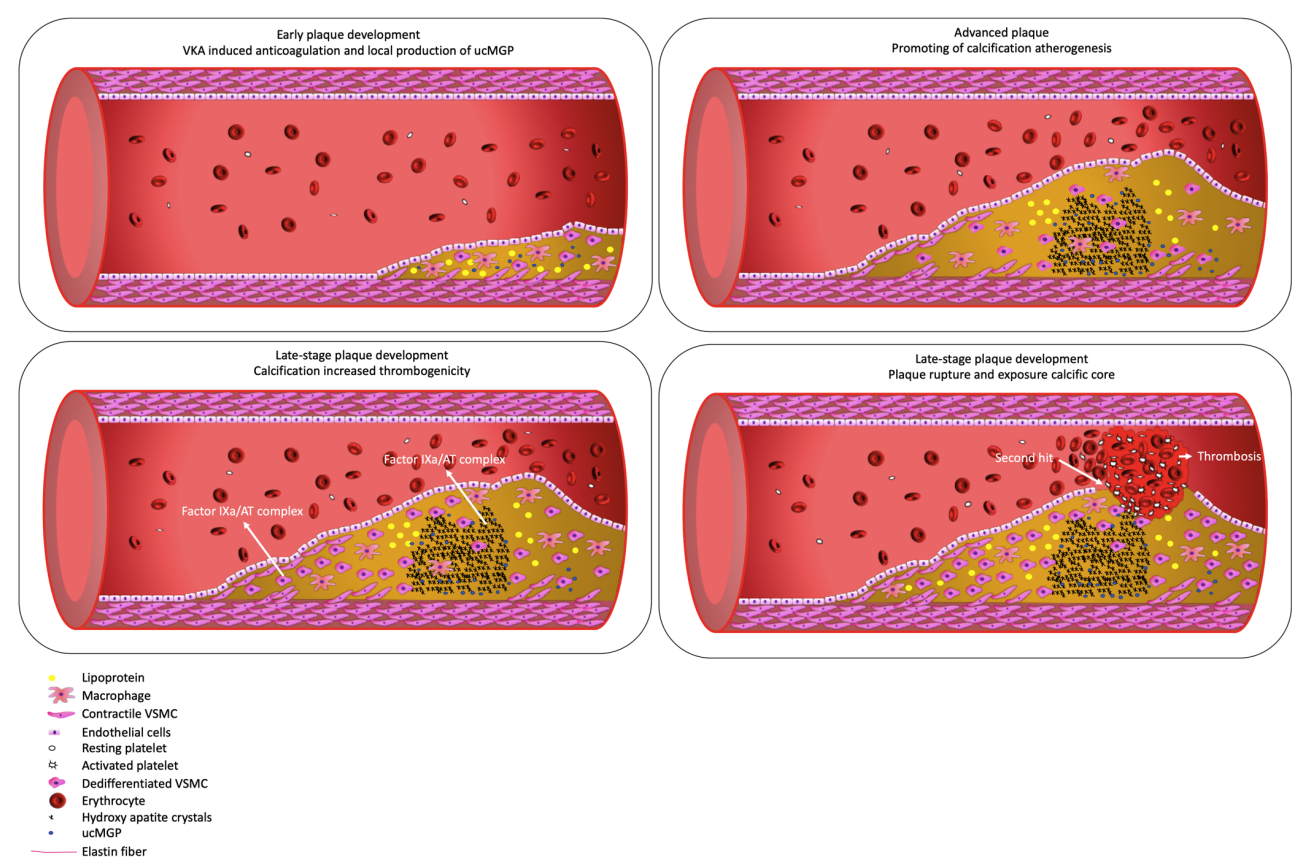

Figure 7. Schematic overview of VKA and calcification on atherothrombosis. Short-term treatment of VKA induces systemic and local vitamin $\mathrm{K}$ deficiency. Long-term VKA treatment aggravates atherogenesis and results in increased vascular calcification. Vascular calcification induces a prethrombotic state priming for an increased risk on atherothrombosis.

In conclusion, our results show that long-term VKA treatment aggravates calcification and atherosclerotic plaque progression (Figure 7). VSMC phenotype switching promotes calcification and TF-dependent coagulation. Calcification of atherosclerotic plaques might be a lesion stabilizing process but it also renders the lesion more prone to cause atherothrombosis following rupture of the plaque. Our findings provide additional support to 
the observed association between CAC and risk for future cardiovascular events.

\title{
Acknowledgements
}

\author{
This work was financed by Dutch Thrombosis Society (2014.02) and by the Norwegian \\ Research Council and Nattopharma ASA. CCB acknowledges funding from the Alexander \\ von Humbold Foundation.
}

\section{References}

1. Budoff MJ, Dowe D, Jollis JG, Gitter M, Sutherland J, Halamert E, et al. Diagnostic performance of 64multidetector row coronary computed tomographic angiography for evaluation of coronary artery stenosis in individuals without known coronary artery disease: results from the prospective multicenter ACCURACY (Assessment by Coronary Computed Tomographic Angiography of Individuals Undergoing Invasive Coronary Angiography) trial. J Am Coll Cardiol. 2008 Nov 18;52(21):1724-32.

2. Rennenberg RJMW, Kessels AGH, Schurgers LJ, van Engelshoven JMA, de Leeuw PW, Kroon AA. Vascular calcifications as a marker of increased cardiovascular risk: a meta-analysis. Vasc Health Risk Manag. 2009;5(1):185-97.

3. Virmani R, Kolodgie FD, Burke AP, Farb A, Schwartz SM. Lessons from sudden coronary death: a comprehensive morphological classification scheme for atherosclerotic lesions. Arterioscler Thromb Vasc Biol. 2000 May;20(5):1262-75.

4. Raggi P, Callister TQ, Shaw LJ. Progression of coronary artery calcium and risk of first myocardial infarction in patients receiving cholesterol-lowering therapy. Arterioscler Thromb Vasc Biol. American Heart Association, Inc; $2004 \mathrm{Jul} ; 24(7): 1272-7$.

5. Irkle A, Vesey AT, Lewis DY, Skepper JN, Bird JLE, Dweck MR, et al. Identifying active vascular microcalcification by (18)F-sodium fluoride positron emission tomography. Nat Comms. 2015;6:7495.

6. Pawade TA, Cartlidge TRG, Jenkins WSA, Adamson PD, Robson P, Lucatelli C, et al. Optimization and Reproducibility of Aortic Valve 18F-Fluoride Positron Emission Tomography in Patients With Aortic Stenosis. Circ Cardiovasc Imaging. 2016 Oct;9(10).

7. Libby P. How does lipid lowering prevent coronary events? New insights from human imaging trials. Eur Heart J. 2015 Feb 21;36(8):472-4.

8. Criqui MH, Knox JB, Denenberg JO, Forbang NI, McClelland RL, Novotny TE, et al. Coronary Artery Calcium Volume and Density: Potential Interactions and Overall Predictive Value: The Multi-Ethnic Study of Atherosclerosis. JACC Cardiovasc Imaging. 2017 Aug;10(8):845-54.

9. Libby P. Mechanisms of acute coronary syndromes and their implications for therapy. N Engl J Med. 2013 May 23;368(21):2004-13.

10. Chatrou MLL, Winckers K, Hackeng TM, Reutelingsperger CP, Schurgers LJ. Vascular calcification: the price to pay for anticoagulation therapy with vitamin K-antagonists. Blood Rev. 2012 Jul;26(4):155-66.

11. Eggebrecht L, Prochaska JH, Schulz A, Arnold N, Jünger C, Göbel S, et al. Intake of Vitamin K Antagonists and Worsening of Cardiac and Vascular Disease: Results From the Population-Based Gutenberg Health Study. J Am Heart Assoc. 2018 Sep 4;7(17):1396-25.

12. Schurgers LJ, Joosen IA, Laufer EM, Chatrou MLL, Herfs M, Winkens MHM, et al. Vitamin Kantagonists accelerate atherosclerotic calcification and induce a vulnerable plaque phenotype. PLoS ONE. 2012;7(8):e43229.

13. Borissoff JI, Joosen IA, Versteylen MO, Spronk HM, Cate ten H, Hofstra L. Accelerated in vivo thrombin formation independently predicts the presence and severity of $\mathrm{CT}$ angiographic coronary atherosclerosis. JACC Cardiovasc Imaging. 2012 Dec;5(12):1201-10.

14. Price PA, Kaneda Y. Vitamin K counteracts the effect of warfarin in liver but not in bone. Thromb Res. 1987 Apr 1;46(1):121-31.

15. Weijs B, Blaauw Y, Rennenberg RJMW, Schurgers LJ, Timmermans CCMM, Pison L, et al. Patients using vitamin $\mathrm{K}$ antagonists show increased levels of coronary calcification: an observational study in low-risk atrial fibrillation patients. Eur Heart J. 2011 Oct;32(20):2555-62.

16. Peeters F, Dudink E, Kimenai D, Weijs B, Altintas S, Heckman L, et al. Vitamin K Antagonists, NonVitamin K Antagonist Oral Anticoagulants, and Vascular Calcification in Patients with Atrial Fibrillation. TH Open. Georg Thieme Verlag KG; 2018 Oct 1;02(04):e391-8.

17. Hobbelt AH, Spronk HM, Crijns HJGM, Cate ten H, Rienstra M, Van Gelder IC. Prethrombotic State in Young Very Low-Risk Patients With Atrial Fibrillation. J Am Coll Cardiol. 2017 Apr 18;69(15):1990-2. 
18. Schurgers LJ, Teunissen KJF, Knapen MHJ, Kwaijtaal M, van Diest R, Appels A, et al. Novel conformation-specific antibodies against matrix gamma-carboxyglutamic acid (Gla) protein: undercarboxylated matrix Gla protein as marker for vascular calcification. Arterioscler Thromb Vasc Biol. 2005 Aug;25(8):1629-33.

19. Reynolds JL, Joannides AJ, Skepper JN, McNair R, Schurgers LJ, Proudfoot D, et al. Human vascular smooth muscle cells undergo vesicle-mediated calcification in response to changes in extracellular calcium and phosphate concentrations: a potential mechanism for accelerated vascular calcification in ESRD. J Am Soc Nephrol. 2004 Nov;15(11):2857-67.

20. Borissoff JI, Heeneman S, Kilinç E, Kassák P, van Oerle R, Winckers K, et al. Early atherosclerosis exhibits an enhanced procoagulant state. Circulation. 2010 Aug 24;122(8):821-30.

21. Kapustin AN, Chatrou MLL, Drozdov I, Zheng Y, Davidson SM, Soong D, et al. Vascular smooth muscle cell calcification is mediated by regulated exosome secretion. Circ Res. Lippincott Williams \& Wilkins; 2015 Apr 10;116(8):1312-23.

22. Loeffen R, Kleinegris M-CF, Loubele STBG, Pluijmen PHM, Fens D, van Oerle R, et al. Preanalytic variables of thrombin generation: towards a standard procedure and validation of the method. $\mathrm{J}$ Thromb Haemost. Wiley/Blackwell (10.1111); 2012 Dec;10(12):2544-54.

23. Van Kruchten R, Cosemans JMEM, Heemskerk JWM. Measurement of whole blood thrombus formation using parallel-plate flow chambers - a practical guide. Platelets. 2012;23(3):229-42.

24. Coppin L, Najimi M, Bodart J, Rouchon M-S, van der Smissen P, Eeckhoudt S, et al. Clinical Protocol to Prevent Thrombogenic Effect of Liver-Derived Mesenchymal Cells for Cell-Based Therapies. Cells. Multidisciplinary Digital Publishing Institute; 2019 Aug 7;8(8):846.

25. Swieringa F, Baaten CCFMJ, Verdoold R, Mastenbroek TG, Rijnveld N, van der Laan KO, et al. Platelet Control of Fibrin Distribution and Microelasticity in Thrombus Formation Under Flow. Arterioscler Thromb Vasc Biol. Lippincott Williams \& Wilkins Hagerstown, MD; 2016 Apr;36(4):692-9.

26. Baaten CCFMJ, Swieringa F, Misztal T, Mastenbroek TG, Feijge MAH, Bock PE, et al. Platelet heterogeneity in activation-induced glycoprotein shedding: functional effects. Blood Adv. 2018 Sep 25;2(18):2320-31.

27. Shearer MJ, Newman P. Metabolism and cell biology of vitamin K. Thromb Haemost. 2008 Oct;100(4):530-47.

28. Lacolley P, Regnault V, Segers P, Laurent S. Vascular Smooth Muscle Cells and Arterial Stiffening: Relevance in Development, Aging, and Disease. Physiol Rev. American Physiological Society; 2017 Oct 1;97(4):1555-617.

29. Schurgers LJ, Akbulut AC, Kaczor DM, Halder M, Koenen RR, Kramann R. Initiation and Propagation of Vascular Calcification Is Regulated by a Concert of Platelet- and Smooth Muscle Cell-Derived Extracellular Vesicles. Front Cardiovasc Med. Frontiers; 2018 Apr 6;5:14391-13.

30. Andrews J, Psaltis PJ, Bayturan O, Shao M, Stegman B, Elshazly M, et al. Warfarin Use Is Associated With Progressive Coronary Arterial Calcification: Insights From Serial Intravascular Ultrasound. JACC Cardiovasc Imaging. 2018 Sep;11(9):1315-23.

31. Win TT, Nakanishi R, Osawa K, Li D, Susaria SS, Jayawardena E, et al. Apixaban versus warfarin in evaluation of progression of atherosclerotic and calcified plaques (prospective randomized trial). Am Heart J. 2019 Mar 13;212:129-33.

32. Puri R, Nicholls SJ, Shao M, Kataoka Y, Uno K, Kapadia SR, et al. Impact of statins on serial coronary calcification during atheroma progression and regression. J Am Coll Cardiol. 2015 Apr 7;65(13):127382.

33. Reutelingsperger C, Schurgers L. Coronary Artery Calcification: A Janus-Faced Biomarker? JACC Cardiovasc Imaging. 2018 Sep;11(9):1324-6.

34. Viegas CSB, Rafael MS, Enriquez JL, Teixeira A, Vitorino R, Luís IM, et al. Gla-Rich Protein Acts as a Calcification Inhibitor in the Human Cardiovascular System. Arterioscler Thromb Vasc Biol. 2014 Dec 23.

35. Kapustin AN, Schoppet M, Schurgers LJ, Reynolds JL, McNair R, Heiss A, et al. Prothrombin Loading of Vascular Smooth Muscle Cell-Derived Exosomes Regulates Coagulation and CalcificationHighlights. Arterioscler Thromb Vasc Biol. American Heart Association, Inc; 2017 Mar 1;37(3):e22-e32.

36. Luo G, Ducy P, McKee MD, Pinero GJ, Loyer E, Behringer RR, et al. Spontaneous calcification of arteries and cartilage in mice lacking matrix GLA protein. Nature. 1997 Mar 6;386(6620):78-81.

37. Parker BD, Schurgers LJ, Brandenburg VM, Christenson RH, Vermeer C, Ketteler M, et al. The associations of fibroblast growth factor 23 and uncarboxylated matrix Gla protein with mortality in coronary artery disease: the Heart and Soul Study. Ann Intern Med. American College of Physicians; 2010 May 18;152(10):640-8. 
38. Joshi NV, Vesey AT, Williams MC, Shah ASV, Calvert PA, Craighead FHM, et al. 18F-fluoride positron emission tomography for identification of ruptured and high-risk coronary atherosclerotic plaques: a prospective clinical trial. Lancet. 2014 Feb 22;383(9918):705-13.

39. Budoff MJ, Young R, Burke G, Jeffrey Carr J, Detrano RC, Folsom AR, et al. Ten-year association of coronary artery calcium with atherosclerotic cardiovascular disease (ASCVD) events: the multi-ethnic study of atherosclerosis (MESA). Eur Heart J. 2018 Jul 1;39(25):2401-8.

40. Mackman N. Triggers, targets and treatments for thrombosis. Nature. Nature Publishing Group; 2008 Feb 21;451(7181):914-8.

41. Furie B, Furie BC. Mechanisms of thrombus formation. N Engl J Med. Massachusetts Medical Society; 2008 Aug 28;359(9):938-49.

42. Ruggeri ZM. Platelets in atherothrombosis. Nat Med. Nature Publishing Group; 2002 Nov;8(11):122734.

43. Colhoun HM, Zito F, Norman Chan N, Rubens MB, Fuller JH, Humphries SE. Activated factor XII levels and factor XII 46C $>$ T genotype in relation to coronary artery calcification in patients with type 1 diabetes and healthy subjects. Atherosclerosis. 2002 Aug;163(2):363-9.

44. Horn P, Erkilet G, Veulemans V, Kröpil P, Schurgers L, Zeus T, et al. Microparticle-Induced Coagulation Relates to Coronary Artery Atherosclerosis in Severe Aortic Valve Stenosis. PLoS ONE. Public Library of Science; 2016;11(3):e0151499.

45. Borissoff JI, Otten JJT, Heeneman S, Leenders P, van Oerle R, Soehnlein O, et al. Genetic and pharmacological modifications of thrombin formation in apolipoprotein e-deficient mice determine atherosclerosis severity and atherothrombosis onset in a neutrophil-dependent manner. PLoS ONE. 2013;8(2):e55784.

46. Spronk HMH, de Jong AM, Verheule S, de Boer HC, Maass AH, Lau DH, et al. Hypercoagulability causes atrial fibrosis and promotes atrial fibrillation. Eur Heart J. The Oxford University Press; 2016 Apr $12 ;:$ ehw119.

47. Mackman N. The role of tissue factor and factor VIIa in hemostasis. Anesth Analg. 2009 May;108(5):1447-52.

48. Taubman MB, Wang L, Miller C. The role of smooth muscle derived tissue factor in mediating thrombosis and arterial injury. Thromb Res. 2008;122 Suppl 1:S78-81.

49. Verhoef JJF, Barendrecht AD, Nickel KF, Dijkxhoorn K, Kenne E, Labberton L, et al. Polyphosphate nanoparticles on the platelet surface trigger contact system activation. Blood. American Society of Hematology; 2017 Mar 23;129(12):1707-17.

50. Wang L, Miller C, Swarthout RF, Rao M, Mackman N, Taubman MB. Vascular smooth muscle-derived tissue factor is critical for arterial thrombosis after ferric chloride-induced injury. Blood. American Society of Hematology; 2009 Jan 15;113(3):705-13.

51. Stampfuss J-J, Censarek P, Fischer JW, Schrör K, Weber A-A. Rapid release of active tissue factor from human arterial smooth muscle cells under flow conditions. Arterioscler Thromb Vasc Biol. American Heart Association, Inc; 2006 May;26(5):e34-7.

52. Breyne J, Juthier F, Corseaux D, Marechaux S, Zawadzki C, Jeanpierre E, et al. Atherosclerotic-like process in aortic stenosis: activation of the tissue factor-thrombin pathway and potential role through osteopontin alteration. Atherosclerosis. $2010 \mathrm{Dec} ; 213(2): 369-76$.

53. Cao Y, Zhou X, Liu H, Zhang Y, Yu X, Liu C. The NF- $\kappa$ B pathway: regulation of the instability of atherosclerotic plaques activated by Fg, Fb, and FDPs. Mol Cell Biochem. Springer US; 2013 Nov;383(12):29-37. 


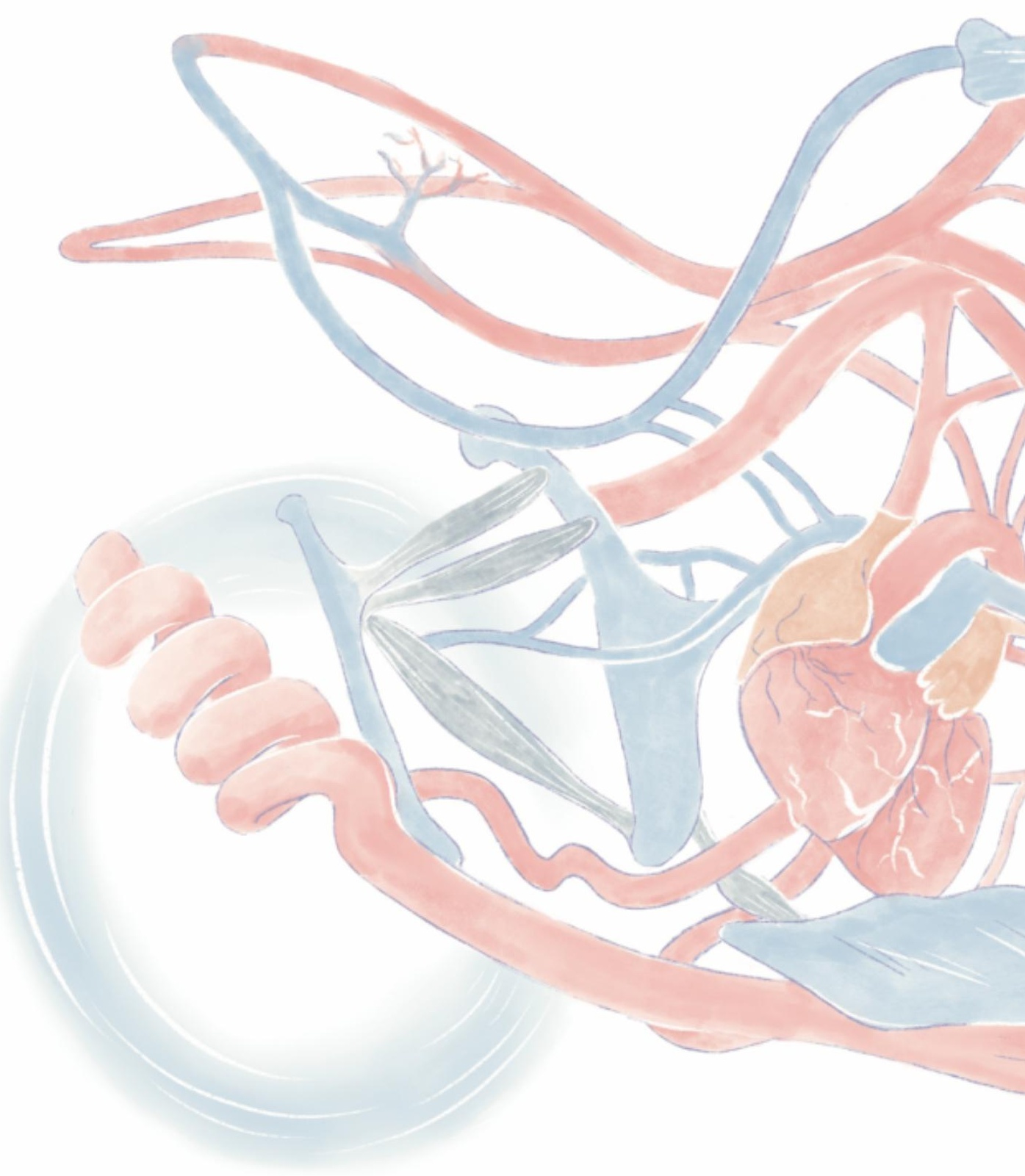




\section{Chapter 4}

Vitamin $\mathrm{K}$ inhibits vascular smooth muscle cell induced calcification via reduced oxidative stress and extracellular vesicle secretion

RH. van Gorp, P Petsophonsakul, AM Jaminon, P Leenders, W Jahnen-Dechent, CP Reutelingsperger, LJ Schurgers. 


\begin{abstract}
Introduction: Vascular calcification is a strong predictor of cardiovascular outcome. Vascular calcification is in part mediated via loss of vitamin $\mathrm{K}$ dependent calcification inhibitor matrix Gla protein (MGP). Vitamin K supplementation has been shown to protect development of medial calcification and thus provides a promising intervention potential. However, the effect of vitamin $\mathrm{K}$ on intimal calcification remains unknown. Therefore, we questioned whether vitamin $\mathrm{K}$ supplementation holds progression of atherosclerotic plaque calcification.
\end{abstract}

Method: Female Apoe ${ }^{-/}$mice were placed on western type diet (WTD) or WTD supplemented with warfarin (Warfarin) for 18 weeks. Additionally, after 6 weeks of initial warfarin supplementation diet was substituted to WTD without (Ctrl) or with vitamin K2 (MK7). Aortic arches were used to assess effects of vitamin K on atherosclerotic plaque development and calcification by (immuno)histochemical analyses. Moreover, in vitro effects of warfarin and MK7 on vascular smooth muscle cells (VSMC) were determined.

Results: Warfarin induced local vitamin K deficiency as shown by significant elevated vascular uncarboxylated MGP, which was accompanied by significantly increased intimal atherosclerotic plaque size and calcification compared to WTD. Substituting warfarin to Ctrl or MK7 significantly attenuated atherosclerotic calcification. Moreover, switching to MK7 significantly reduced plaque size and oxidative stress compared to continuing warfarin, while increasing collagen content of atherosclerotic plaques compared to Ctrl. Exogenous injected fetuin-A-alexa488, a fluorescent calcification binding probe, showed significant reduced atherosclerotic plaque uptake after MK7 compared to warfarin and Ctrl. In vitro, MK7 reduced and attenuated warfarin induced VSMC oxidative stress and extracellular vesicle release and subsequent calcification.

Conclusion: VKA induces vitamin $\mathrm{K}$ deficiency and aggravates atherosclerosis and calcification. Stopping VKA treatment resulted in less atherosclerosis and calcification. Switching to MK7 supplementation reduced atherosclerotic plaque progression and improved markers of plaque stability. The beneficial effects of restoring vitamin $\mathrm{K}$ status in the vessel wall are probably mediated by direct effects on VSMC. Our data support clinical trials testing the hypothesis that vitamin $\mathrm{K}$ treatment can hold progression of atherosclerosis and vascular calcification. 


\section{Introduction}

Vascular calcification is associated with increased risk of all-cause cardiovascular mortality (1). Coronary artery calcification (CAC) develops with atherosclerosis and is measured to predict cardiovascular events (2). In an observational study of 25,253 asymptomatic patients, a CAC score higher than 1,000 was correlated with a 16\% lower 10 year survival rate, even after the adjustments for risk factors, such as age, hypercholesterolemia, diabetes, smoking, hypertension, and a family history of premature coronary heart disease (3). Additionally, annual increase of vascular calcification predicts cardiovascular events $(4,5)$. Location, size and activity of vascular calcification determine to worsening cardiovascular outcome. Micro- and metabolic active calcification increase plaque vulnerability and thus risk on myocardial infarction and stroke (6).

While initially thought of as a passive process, vascular calcification is now accepted to be highly regulated by vascular smooth muscle cells (VSMC) and calcification inhibitory proteins. Vitamin K antagonist (VKA), used as antithrombotic agent, is associated with increased vascular calcification (7). VKA induce vascular calcification in part by loss of vitamin K-dependent calcification inhibitor matrix Gla protein (MGP) (8). The function of MGP was highlighted in MGP deficient mice, which developed severe vascular calcification leading to death by ruptured vessels within 6 weeks of birth (9). In vivo mutagenesis experiments showed that the calcification inhibitory function of MGP requires the Glaresidues (10), which are formed with the aid of vitamin $\mathrm{K}$ and thus inhibited by VKA. Elevated uncarboxylated MGP (ucMGP) is localized around areas of vascular calcification, suggesting lack of inhibition (11). Also increased circulating levels of inactive MGP have been shown to correlate with vascular calcification $(12,13)$ and cardiovascular mortality $(14$, $15)$.

An extensive list of potential medications that address vascular calcification has been compiled (16). However, most of these have only been studied in preclinical models, lack clinical research or have serious side effects. Of all enlisted drugs, vitamin $\mathrm{K}$ seem to have great potential considering that it is safe and cost-effective for inhibiting vascular calcification. MGP requires vitamin K unequivocally for its activity. Indeed, high vitamin K supplementation has been shown to stabilize or inhibit medial calcification in pre-clinical models of VKA-induced calcification $(8,17)$. Data from epidemiological studies have pointed out an important role of vitamin $\mathrm{K}$ as a potentially protective factor for cardiovascular health. People with the highest intake of food rich in vitamin K2 had a significantly reduces cardiovascular risk and vascular calcification (18-20).

Increasing dietary vitamin $\mathrm{K}$ intake or oral vitamin $\mathrm{K}$ supplementation is relatively easy and cost effective and thus it is intriguing to speculate about a favorable risk-benefit ratio. Vitamin K supplementation appears to be safe and the WHO has set no upper tolerance level for vitamin $\mathrm{K}$ intake (21). Indeed, vitamin $\mathrm{K}$ has been shown to hold progression of vascular (22) and valvular (23) calcification. Vitamin K2 subtype menaquinone-7 (MK7) has 
been shown to dose-dependently reduce inactive MGP concentrations $(24,25)$. Currently, several clinical trials are testing the hypothesis whether MK7 supplementation holds progression of CAC (VitaK-CAC (NCT01002157) and BASIK2 (NCT02917525)). Here, we studied the effect of vitamin K (MK7) supplementation on atherosclerotic plaque development and calcification.

\section{Materials and methods}

Animals. All animal studies were performed under an approved protocol by the ethics committee for animal experiments of Maastricht University. Twelve-week-old female C57BL6 Apoe ${ }^{-/-}$mice were purchased from Charles River (Den Bosch, The Netherlands) and housed in climate-controlled spaces under 12-hour day/night cycle with ad libitum access to food and water. All mice were fed an irradiated (0.9Mrad) vitamin K-deficient Western Type Diet (WTD: $0.25 \%$ cholesterol and 15\% cocoa butter, derived from Altromin, Germany). WTD was supplemented with in corn oil dissolved vitamin K1 (5 $\mathrm{g} / \mathrm{g}$; Merck KGaA, Darmstadt, Germany) for control, warfarin (3 mg/g warfarin, Merck KGaA, Darmstadt, Germany) and vitamin K1 (1.5 mg/g) for VKA treatment or vitamin K2 (MK7; $100 \mu \mathrm{g} / \mathrm{g}$, kindly supplied by Nattopharma, Oslo, Norway) for vitamin K supplementation. Vitamin $\mathrm{K} 1$, antagonises the effect of warfarin in liver, yet has little to no effect in extrahepatic tissue (26). Therefore, vitamin K1 is co-administered with warfarin to prevent bleedings, yet introducing vitamin K-deficiency in the vasculature (27).

Mice were randomly assigned to receive WTD without (WTD) or with warfarin (Warfarin) for 18 weeks. After 6 weeks of warfarin diet, part of the animals was placed on WTD (Ctrl) or WTD supplemented with MK7 (MK7) for another 12 weeks of treatment (Figure 1A). Mice that received warfarin were intraperitoneally injected with fetuin-A-Alexa488 24 hours before sacrificing.

After treatment periods, mice were sacrificed and blood was collected via the portal vein in $105 \mathrm{mM}$ trisodium citrate and plasma aliquots were frozen at $-80^{\circ} \mathrm{C}$ until analysis. Next, the vasculature was washed with a sterile binding buffer $(40 \mathrm{mM}$ Hepes, $150 \mathrm{mM} \mathrm{NaCl}, 5 \mathrm{mM}$ $\mathrm{KCl}, 1 \mathrm{mM} \mathrm{MgCl}_{2}$ and $2.5 \mathrm{mM} \mathrm{CaCl}_{2}, \mathrm{pH} 7.3$ ) via the left ventricle. The aortic arch was dissected and fixated overnight in $1 \%(\mathrm{v} / \mathrm{v})$ HEPES-buffered formaldehyde containing $150 \mathrm{mM}$ saline at $4^{\circ} \mathrm{C}$ before being embedded in paraffin. Sections were cut at $4 \mu \mathrm{m}$ thickness.

Blood analysis. Total plasma cholesterol was assessed with the Synchron LX20 (Beckman Coulter, Brea, USA). Vitamin K concentration in plasma was determined using HPLC as described previously (28).

Histochemical analysis. Plaque size was determined by hematoxyline and eosine (HE) stain and analysed using ImageJ software. Plaques were analysed for calcification (alizarin Red S), collagen (picro Sirius red), necrotic core (Toluidine blue) and number of elastin breaks (Elastica von Gieson). Elastin breaks found in the medial layer beneath the atherosclerotic plaque were scored using a scoring system ranging from 0 to $4 ; 0$, no breaks; 1 , one-three breaks; 2, four-six breaks; 3, seven-nine breaks and 4, $9>$ breaks. 
Immunohistochemistry was used to further examine plaque phenotype. Plaques were assessed for uncarboxylated matrix Gla protein (ucMGP, 1:400; IDS, Boldon, UK), carboxylated MGP (cMGP, 1:400; IDS, Boldon, UK), oxidative stress (8-hydroxyguanisine 1:300; Meridian Life Science, Tennessee, USA), alpha-SMA (1:400, Merck KGaA, Darmstadt, Germany), MAC3 (1:50, Biosciences, San Jose, United States) and to visualize injected fetuin-A-488 (anti-Alexa488 1:5000; Invitrogen, California, USA). HRP conjugated secondary antibodies were visualized with Nova-RED substrate (Vector Labs, Amsterdam, the Netherlands). 2D analysis quantification was performed using Leica Application Suite X (Leica Microsystems, Wetzlar, Germany).

Vascular smooth muscle cells. Human primary vascular smooth muscle cells (VSMC) were obtained from human aortic tissue explants obtained from males and females aged between 18 and 65 years of age. VSMCs were cultured in DMEM medium (Gibco, Thermo Fisher Scientific Inc, Paisley, Scotland, UK) supplemented with 20\% fetal bovine serum (FBS), 100 $\mathrm{U} / \mathrm{mL}$ penicillin and $100 \mu \mathrm{g} / \mathrm{mL}$ streptomycin. Cells were used between passage 5 and 10 . qPCR. RNA extraction, cDNA synthesis and qPCR were performed as described previously (29). The following primers were used: Nrf2 forward; 5'CAGCGACGGAAAGAGTATGA-3, Nrf2 reverse; 5'-TGGGCAACCTGGGAGTAG-3, GAPDH forward; 5'-AACGGATTTGGTCGTATTGGGC-3, GAPDH reverse; 5'CTTGACGGTGCCATGGAATTTG-3.

In vitro calcification measurement. VSMC were plated in a 24 well plates to adhere overnight in culture medium. Next, VSMC were placed in calcifying medium consisting of DMEM supplemented with $2.5 \% \mathrm{FBS}, 100 \mathrm{U} / \mathrm{mL}$ penicillin, and $100 \mu \mathrm{g} / \mathrm{mL}$ streptomycin in the presence of an additional $1.8 \mathrm{mmol} / \mathrm{L}$ calcium in addition of stimulus. Calcium deposits were assessed by O-cresolphthalein and calcium content was normalized for protein content (micro BSA protein kit, Thermo Fisher Scientific Inc, Paisley, Scotland, UK).

Extracellular oxidative stress in VSMC. To determine oxidative stress, we measured extracellular ROS using Amplex Red, which detects hydrogen peroxide or peroxidase activity. VSMC were seeded in a 96-well which were allowed to adhere overnight. After 24 hours of treatment, cells were washed twice and placed in the presence of stimulus in KrebsRinger Phosphate Glucose buffer (KRPG; $145 \mathrm{mM} \mathrm{NaCl}, 5.7 \mathrm{mM}$ sodium phosphate, 4.86 $\mathrm{mM} \mathrm{KCl}, 0.54 \mathrm{mM} \mathrm{CaCl}$, 1.22mM MgSO4, 5.5mM glucose, $\mathrm{pH}$ 7.4) mixed 1:1 with Amplex Red mixture (50 $\mu \mathrm{M}$ Amplex Red (Thermo Fisher Scientific Inc, Waltham, MA, USA) and $0.1 \mathrm{U} / \mathrm{ml}$ HRP. Fluorescence (excitation wavelength 544nm; emission wavelength 590nm) was measured using the Cytation 3 Cell Imaging Multi-Mode Reader (BioTek Instruments Inc., Winooski, VT, USA). To normalize for cell number, total protein content was measured using a micro BCA protein assay kit from Thermo Scientific (Thermo Fisher Scientific Inc, Waltham, MA, USA) according to the manufacturer's instructions.

Intracellular VSMC hydrogen peroxide. To identify intracellular ROS, we used the cell permeable fluoregenic substrate 2,7 - dichlorofluorescein diacetate (DCFDA, Merck KGaA, 
Darmstadt, Germany) which is oxidized to 2,7 -dichlorofluorescein in the presence of reactive oxidants.

VSMC were seeded in a 96-well plate and left to adhere overnight. Next, culture medium was replaced by Krebs-Ringer Phosphate Glucose buffer (KRPG; 145mM NaCl, 5.7mM sodium phosphate, $4.86 \mathrm{mM} \mathrm{KCl}, 0.54 \mathrm{mM} \mathrm{CaCl} 2,1.22 \mathrm{mM} \mathrm{MgSO} 4,5.5 \mathrm{mM}$ glucose, $\mathrm{pH}$ 7.4) in the presence of $20 \mu \mathrm{M}$ DCFDA for $60 \mathrm{~min}$ in the dark at $37^{\circ} \mathrm{C}$ and $5 \% \mathrm{CO}$. After washing, cells were incubated with stimulus in combination with $1 \mu \mathrm{g} / \mathrm{mL}$ Hoechst and fluorescence was measured (Excitation 485, Emission 529) with a Cytation 3 Cell Imaging Multi-Mode Reader (BioTek Instruments Inc., Winooski, VT, USA) for a total of 30 minutes. Fluorescence intensity was corrected for background and normalized to cell count.

Extracellular vesicle quantification. VSMC cells were seeded in a 12-well plate and allowed to adhere overnight. Next, VSMC were placed in low serum conditions $(0.5 \% \mathrm{FBS})$ with stimulus for 24 hours, after which medium was collected. Medium was centrifuged at $3000 \mathrm{x}$ g for 15 minutes and incubated with CD-63 (BD Bioscience, San Jose, United States) coupled beads overnight. After washing with PBS-2\% BSA, beads were incubated with a secondary antibody anti-CD-81-APC (1:50, BD Bioscience, San Jose, United States) and incubated for 60 minutes in the dark. After washing, beads were placed in Hepes buffer with additional $2.5 \mathrm{mM} \mathrm{Ca}^{2+}$ and $250 \mathrm{ng} / \mathrm{mL}$ Alexa-647 labelled AnnexinA5. Hereafter, extracellular vesicles were detected by flow cytometry (Accuri C6, BD bioscience, San Jose, United States). Exosome secretion is expressed as arbitrary units, which were calculated as follow: median fluorescence was multiplied by percentage positive beads and this was normalized for cell number, as described before (29).

Statistical analyses. In vivo data are presented as median with interquartile range or stated otherwise. All in vitro data were obtained in three or more independent experiments in triplicates or more wells and data are expressed as mean with SD. Data were analysed using Mann-Whitney T-test or Kruskal-Wallis One-way ANOVA as indicated using PRISM software (GraphPad 8.3.0, San Diego, US). Values of $\mathrm{p}<0.05$ were considered statistically significant.

\section{Results}

Mice were fed a western type diet (WTD) or WTD supplemented with warfarin (Warfarin) for 18 weeks. Additionally, mice were fed a warfarin diet for 6 weeks, after which the diet was substituted to WTD supplemented without (Ctrl) or with MK7 supplementation (MK7) (Figure 1A, schematic experimental overview). Weight and cholesterol levels were unaltered between groups after 18 weeks of treatment (Table 1). MK7 supplementation was confirmed in plasma of mice. Here, mice receiving MK7 supplementation showed a mean of $69 \mu \mathrm{g} / \mathrm{L} \mathrm{MK} 7$ and $2 \mu \mathrm{g} / \mathrm{L}$ of MK4 levels in blood plasma, while both forms of vitamin K2 were absent in treatment without MK7 supplementation (Table 1). 
Table 1. Model validation.

\begin{tabular}{|c|c|c|c|c|}
\hline & WTD & Warfarin & Ctrl & MK7 \\
\hline Weight $(\mathbf{g})$ & $23.82 \pm 1.6$ & $22.5 \pm 1.93$ & $23.87 \pm 0.99$ & $23.28 \pm 1.81$ \\
\hline Cholesterol $(\mathbf{m M})$ & $21.27 \pm 3.34$ & $20.56 \pm 4.26$ & $19.23 \pm 4.75$ & $19.91 \pm 5.84$ \\
\hline MK7 $(\boldsymbol{\mu g} / \mathbf{L})$ & - & - & - & $69 \pm 11$ \\
\hline MK4 $(\boldsymbol{\mu g} / \mathbf{L})$ & - & - & - & $2 \pm 0.8$ \\
\hline
\end{tabular}

Vitamin K metabolism in atherogenesis and calcification.

First, we tested effect of long term warfarin treatment on the vasculature. After 18 weeks of treatment, calcification was significantly increased in warfarin treated animals compared to WTD control (median of $66 \%$ and $21 \%$ of plaque area, respectively; Figure 1B). Moreover, 18 weeks of warfarin treatment resulted in a significant increase in plaque size compared to WTD (median of $0.40 \mathrm{~mm}^{2}$ vs $0.57 \mathrm{~mm}^{2}$; $\mathrm{p}<0.05$; Figure 1C).

Next, we treated animals for 6 weeks with warfarin, after which mice were split and put on control or MK7 diet for another 12 weeks (Figure 1A). In animals treated for 6 weeks with warfarin, no calcification could be detected histochemically using Alizarin Red S stain (data not shown). Substitution to Ctrl and MK7 showed significant reduction in amount of

A
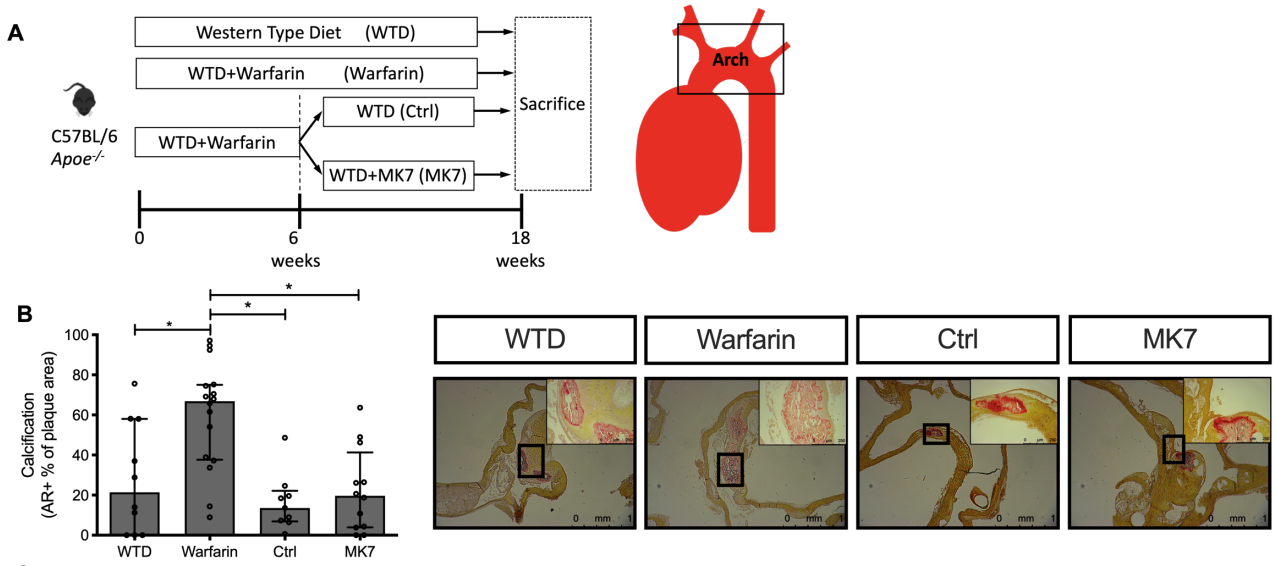

C
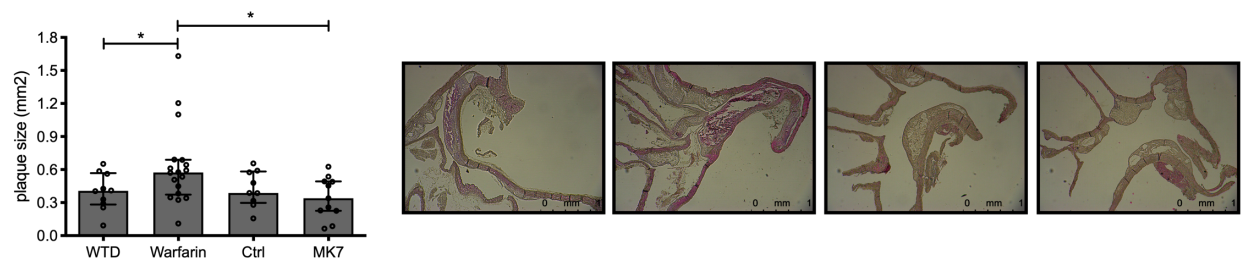

Figure 1. Warfarin increase atherosclerotic calcification and progression after 18 weeks of treatment. A) Schematic overview of animal experiment. B) Warfarin treatment significantly increased calcification compared to WTD treatment. Switching from warfarin to Ctrl or MK7 supplementation significantly reduced calcification to similar levels are WTD. C) Plaque size $\left(\mathrm{mm}^{2}\right)$ was significantly increased after warfarin treatment compared to control. MK7 supplementation significantly reduced plaque size compared to warfarin. Data is presented as median with interquartile range. Statistical significance was assed using Kruskal-Wallis test, * $\mathrm{p}<0.05$, **. 
calcification compared to warfarin and same calcification area as compared to WTD ( $\mathrm{p}<$ 0.05; Figure 1B). Switching from warfarin diet to Ctrl or MK7 resulted in a plaque size similar to WTD $\left(0.38 \mathrm{~mm}^{2}\right.$ plaque size $)$, with a significant attenuation in plaque size of MK7 compared to warfarin $\left(0.33 \mathrm{~mm}^{2}\right.$ plaque size; Figure $\left.1 \mathrm{C}\right)$.

To test for local vitamin $\mathrm{K}$ deficiency, uncarboxylated MGP (ucMGP) in the atherosclerotic plaque was determined. Warfarin treatment for 18 weeks significantly increased ucMGP positivity in the atherosclerotic plaque compared to WTD $(\mathrm{p}<0.001$; Figure 2A). Although switching from warfarin to either control or MK7 reduced ucMGP, no significant difference was found (Figure 2A). In contrast, MK7 significantly increased cMGP in atherosclerotic plaque compared to $\mathrm{Ctrl}(2.9 \%$ vs $0.5 \%$ of plaque area; Figure $2 \mathrm{~B})$. No significant difference in cMGP was observed between warfarin and WTD or Ctrl.

A
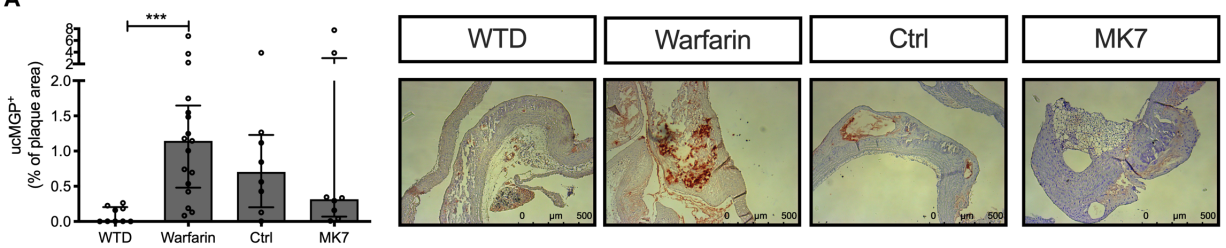

B
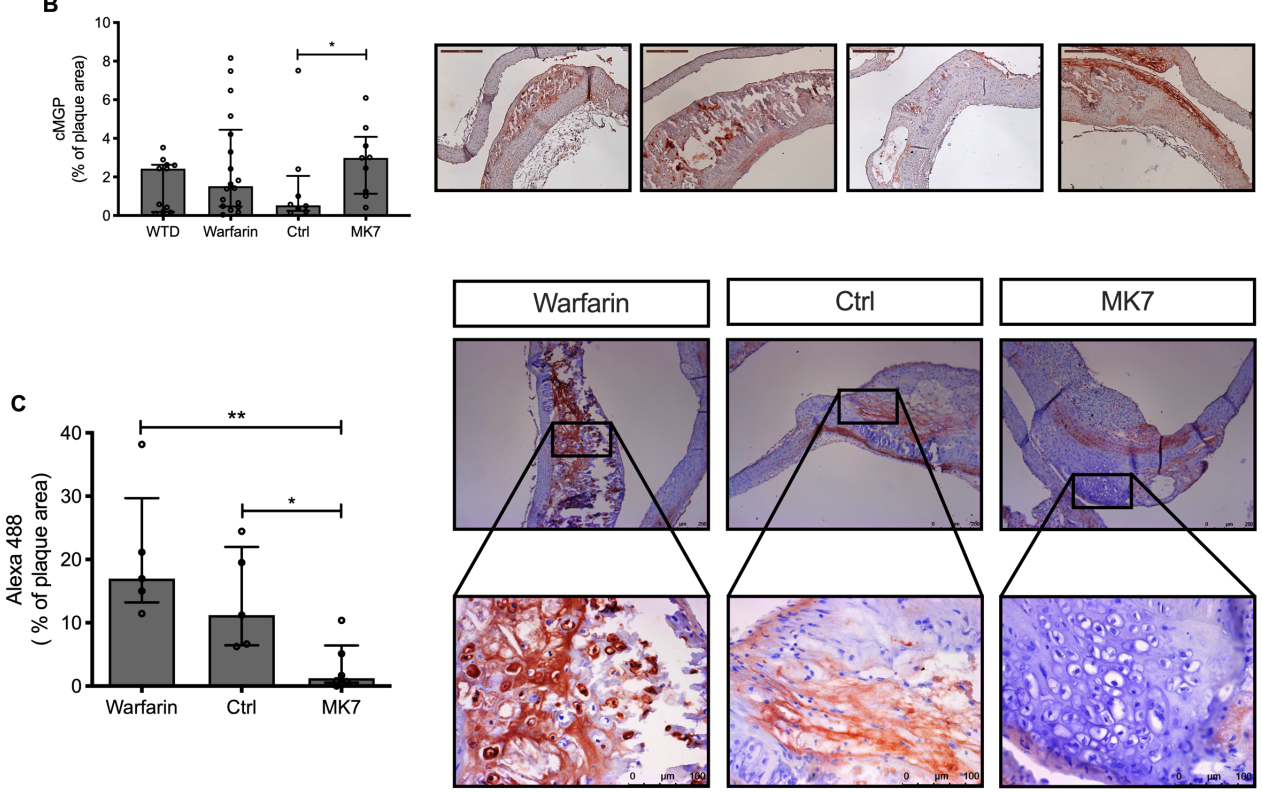

Figure 2. Vitamin $\mathbf{K}$ metabolism in atherosclerotic plaques. A) 18 weeks of warfarin treatment significantly increased uncarobxylated MGP (ucMGP) presence compared to control. No significant difference was found in ucMGP presence after switching to Ctrl or MK7 in atherosclerotic plaques. B) MK7 supplementation significantly increased presence of carboxylated MGP (cMGP) in atherosclerotic plaques compared to Ctrl. C) Visualization of intraperitoneal injected fetuin-A-Alexa488 demonstrated a significant reduction after MK7 supplementation compared to warfarin and Ctrl treatment. Data is presented as median with interquartile range. Statistical significance was assed using Kruskal-Wallis test, * $\mathrm{p}<0.05, * * \mathrm{p}<0.01, * * * \mathrm{p}<0.001$. 
Warfarin treated animals received an intraperitoneal injection of fluorescently labelled fetuin-A-Alexa488, 24 hours before sacrifice. Fetuin-A is a calcification inhibitor with strong affinity for calcium-crystals (30). Moreover, fetuin-A is internalized and secreted by VSMC vesicles $(29,31)$. Fetuin-A-Alexa488 was detected by an HRP-labelled antibody directed against the fluorophore Alexa488. Visualization revealed high presence of fetuin-AAlexa488 in atherosclerotic plaques after warfarin treatment $(20.5 \%$ of plaque volume; Figure 2C). Switching to MK7 showed a significant reduction of fetuin-A presence in atherosclerotic plaques $(1.2 \%$ of plaque volume $)$ compared to warfarin $(\mathrm{p}<0.01)$ and $\mathrm{Ctrl}$ $(11.2 \%$ of plaque volume; $\mathrm{p}<0.05$; Figure $2 \mathrm{C})$. Fetuin-A was also present in the medial and adventitial layer, however this was similar between all treatment.

\section{MK7 promotes plaque stability}

MK7 substitution significantly reduced oxidative stress (as measured by positive 8OHdG stain) in the plaque compared to 18 weeks of warfarin treatment (23\% vs $57 \%$ of 8 $\mathrm{OHdG}+$ cells for MK7 and warfarin respectively; Figure 3A). No difference was observed in intraplaque oxidative stress between WTD and warfarin treated groups after 6 weeks of treatment (data not shown), but was significantly increased in warfarin treated mice after 18 weeks of treatment (Figure 3A). Additionally, elastin breaks in the medial layer under the plaque and collagen content of the plaque were measured as markers of plaque vulnerability.
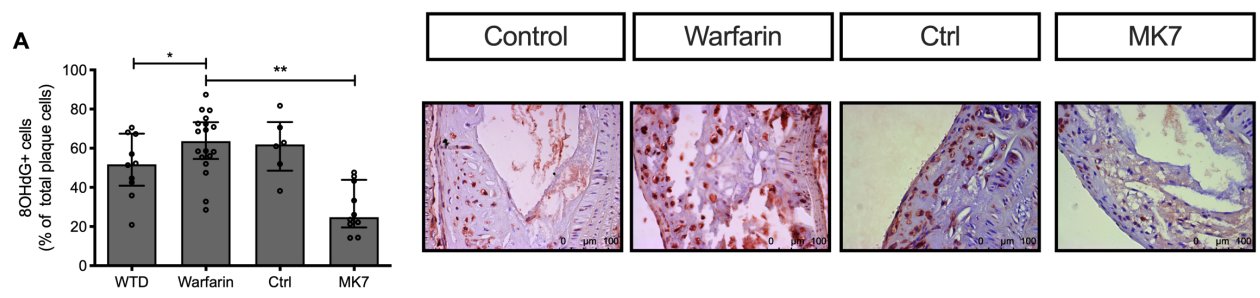

B
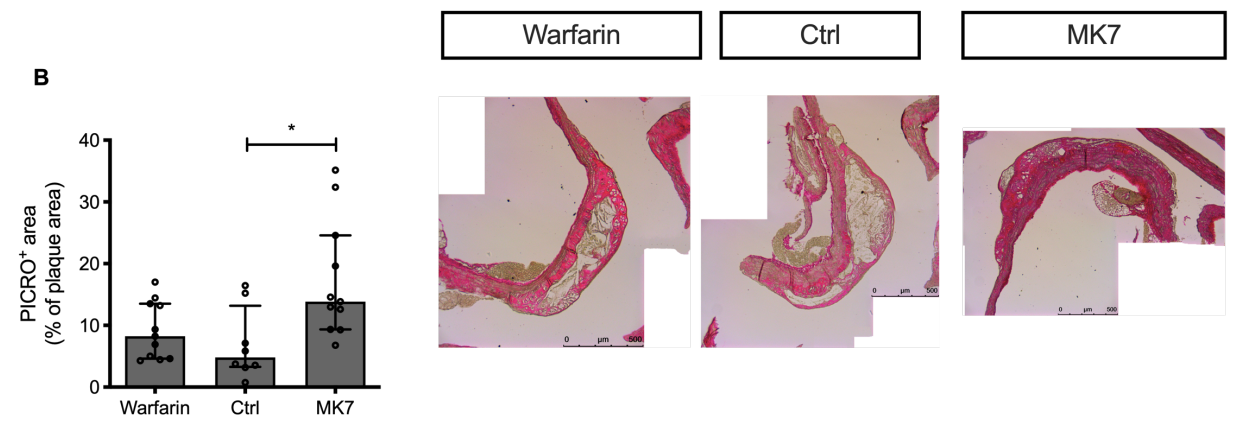

Figure 3. MK7 promotes plaque stability. A) Warfarin significantly increased oxidative stress in the atherosclerotic plaque, as visualized by 8-OhDG, compared to control. MK7 significantly reduced oxidative stress in atherosclerotic plaques compared to 18 weeks of warfarin treatment. B) MK7 treatment significantly increased collagen in atherosclerotic plaques compared to warfarin treatment. Data is presented as median with interquartile range. Statistical significance was assed using Kruskal-Wallis test, ${ }^{*} \mathrm{p}<0.05,{ }^{* *} \mathrm{p}<0.01$. 
Table 2. Plaque characteristics after 18 weeks of treatment.

\begin{tabular}{|c|c|c|c|c|}
\hline \multicolumn{2}{|c}{ WTD } & Warfarin & Ctrl & MK7 \\
\hline Elastin breaks (score) & $1.56 \pm 0.77$ & $2.5 \pm 0.57 *$ & $2.07 \pm 0.44$ & $2.2 \pm 0.6$ \\
\hline Necrotic core (\% of plaque) & $5.8 \pm 4.38$ & $7.91 \pm 10$ & $17.18 \pm 17.1$ & $9.97 \pm 8.48$ \\
\hline $\boldsymbol{\alpha S M A + ~ c e l l s ~ ( \% ~ o f ~ p l a q u e ) ~}$ & $1.82 \pm 1.32$ & $1.46 \pm 1.26$ & $1.48 \pm 1.31$ & $1.11 \pm 0.6$ \\
\hline MAC3+ cells (\% of plaque) & $5.37 \pm 4.1$ & $5.33 \pm 5.28$ & $5.89 \pm 3.98$ & $10.66 \pm 7$ \\
\hline
\end{tabular}

No difference in medial elastin breaks was observed after 6 weeks of treatment between WTD and warfarin treatment (data not shown). However, 18 weeks warfarin treatment significantly increased elastin breaks compared to control $(\mathrm{p}<0.01$; Table 2$)$. No significant difference was observed after switching from warfarin diet to either Ctrl or MK7 supplemented diet. No difference was observed between warfarin and Ctrl in collagen content of atherosclerotic plaques (Figure 3B). Switching to MK7 supplementation increased collagen content in atherosclerotic plaques compared to warfarin $(p=0.099)$ and was significantly increased compared to $\mathrm{Ctrl}(9.1 \%, 6.9 \%$ and $17.4 \%$ of plaque volume for warfarin, Ctrl and MK7 treated groups respectively; Figure 2B). Plaque parameters of necrotic core size, $\alpha \mathrm{SMA}+$ and MAC3+ cells showed no significant difference between any groups (Table 2).

Warfarin treatment of human vascular smooth muscle cells increases calcification.

VSMC have been shown to be pivotal players in vascular calcification and

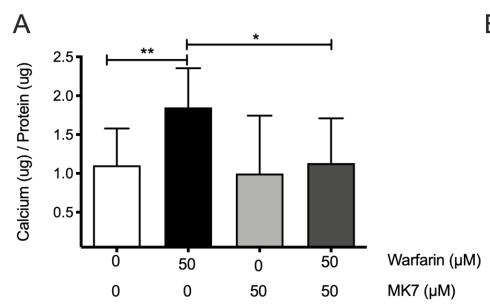

B
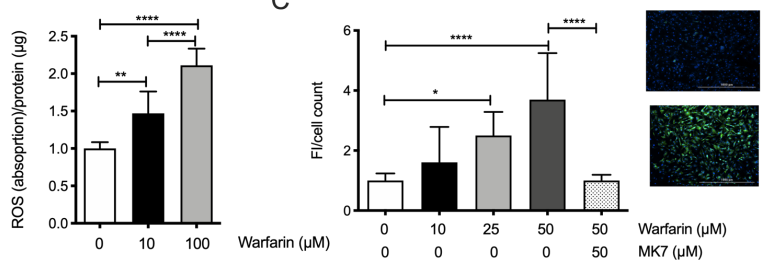
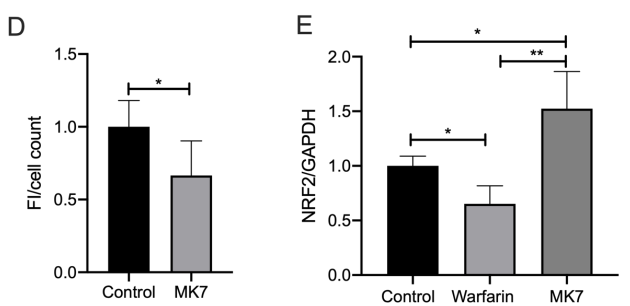

Figure 4. Antioxidant properties of Vitamin $K$ in vascular smooth muscle cells. A) Warfarin treatment increased calcification of VSMC and warfarin induced calcification can be blocked by co-stimulation with MK7. B) Warfarin treatment increased extracellular oxidative stress in a dose dependent manner after 24 hours of stimulation C) Direct warfarin stimulation induced dose-dependent intracellular oxidative stress, which is blocked by co-stimulation with MK7. D) Direct MK7 stimulation reduced intracellular oxidative stress in VSMC compared to control. E) Stimulation of VSMC with warfarin and MK7 reduced and increased Nrf2 mRNA levels, respectively. Data is presented as mean \pm SD. Statistical significance was assed using t-test or Kruskal-Wallis test when applicable, * $p$ $<0.05,{ }^{* *} \mathrm{p}<0.01,{ }^{* * * *} \mathrm{p}<0.0001$. 
atherogenesis development. Therefore, we tested the effect of warfarin and MK7 on VSMC in vitro. Warfarin significantly increased calcification of VSMC compared to vehicle control (Figure 4A). To test whether warfarin induced calcification is vitamin $\mathrm{K}$ dependent, warfarin was cotreated with an equal dose of MK7. Warfarin induced VSMC calcification was completely prevented by MK7 (Figure 4A).

$M K 7$ reduces oxidative stress and protects from warfarin induced ROS.

Switching diet from warfarin to MK7 reduced oxidative stress in atherosclerotic plaques. Therefore, we examined the role of warfarin and MK7 on VSMC oxidative stress. Incubating VSMC for 24 hours with warfarin significantly increased extracellular peroxide levels in a dose dependent manner compared to control treated VSMC (1.4 and 2.1-fold increase for 10 and $100 \mu \mathrm{M}$ warfarin, respectively; Figure 4B). Moreover, incubation of VSMC with warfarin induced a significant dose dependent increase in intracellular ROS (2.5-fold; $\mathrm{p}<$ 0.05 and 3.7-fold; $\mathrm{p}<0.001$ for $25 \mu \mathrm{M}$ and $50 \mu \mathrm{M}$ of warfarin, respectively; Figure 4C). Stimulation of VSMC with a combination of warfarin and MK7 prevented increased intracellular ROS levels ( $\mathrm{p}<0.001$; Figure 4C). Moreover, MK7 significantly reduced intracellular ROS levels compared to control ( $66 \%$ of control levels, $p<0.05$; Figure 4D). The master transcription regulator of oxidative stress is nuclear factor erythroid 2-related factor 2 (Nrf2). Therefore, we assessed the impact of vitamin $\mathrm{K}$ and warfarin on mRNA level of nuclear factor Nrf2. Warfarin stimulation significantly reduced Nrf2 levels compared to control (0.65-fold; p < 0.05), whereas MK7 significantly increased Nrf2 mRNA levels compared to both control $(1.5$-fold; $\mathrm{p}<0.05))$ and warfarin $(\mathrm{p}<0.01)$, respectively (Figure 4E).

MK7 counteracts warfarin induced extracellular vesicle release.

Extracellular vesicles have been associated with vascular calcification by forming a nidus for calcification (29). Reduced fetuin-A presence in plaques of MK7 treated mice might be a result of altered extracellular vesicle uptake or secretion. Presence of fetuin-A in vesicles promotes vesicles phagocytosis and blocks vesicle-mediated VSMC calcification. Therefore, we determined quantity of extracellular vesicles secreted by VSMC when incubated with either warfarin or MK7. After 24 hours of stimulation, warfarin significantly increased extracellular vesicle number in culture medium $(2.2$-fold; $\mathrm{p}<0.01)$, which was inhibited by simultaneous stimulus of MK7 ( $p<0.05$; Figure $5 \mathrm{~A})$. MK7 treatment significantly reduced extracellular vesicle number compared to control (0.86-fold of control; Figure 5B). To test whether phagocytosis potential would be affected, we detected phosphatidylserine on extracellular vesicles using AnxA5-FITC. Warfarin treatment significantly increased phosphatidylserine exposure on extracellular vesicles compared to control and MK7 treatment, while no difference was present between control and MK7 (Figure 5C). 
A

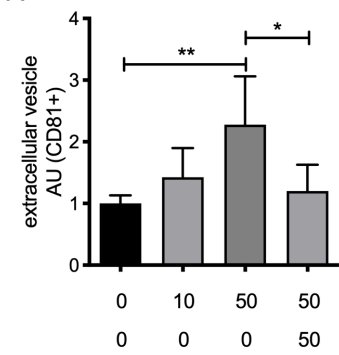

B

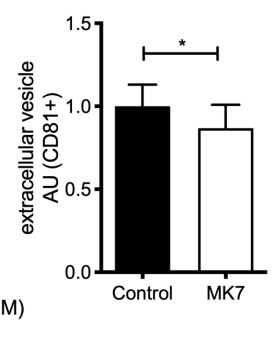

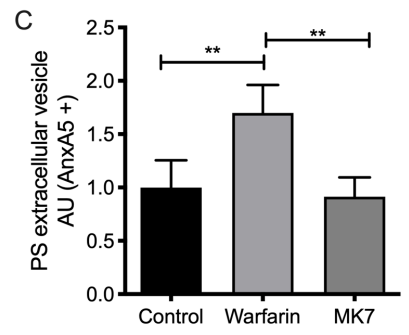

Figure 5. Vitamin K metabolism and extracellular vesicles in vascular smooth muscle cells. A) 24 hours of warfarin treatment increased extracellular vesicles in the medium. Warfarin increased extracellular vesicles numbers was blocked by co-stimulation with MK7. B) MK7 reduced extracellular vesicle numbers compared to control, after 24 hours. C) Warfarin significantly increased phosphatidylserine (PS) exposure on extracellular vesicles compared to control and MK7. Data is presented as mean \pm SD. Statistical significance was assed using t-test or KruskalWallis test when applicable, $* \mathrm{p}<0.05, * * \mathrm{p}<0.01$.

\section{Discussion}

In this paper we show that warfarin aggravates atherosclerosis and calcification and that discontinuing warfarin treatment results in attenuation of both atherosclerosis progression and calcification. Supplementation of MK7 improves plaque stability, in part via direct effects on VSMC by reducing oxidative stress and extracellular vesicle release. Our data support clinical trials that test the hypothesis whether MK7 supplementation has beneficial effects on the cardiovascular system.

Long term warfarin treatment results in increased calcification and plaque progression. In patients, VKA duration is associated with more vascular calcification $(8,32,33)$ and increased intraplaque haemorrhages (34). Additionally, duration of VKA treatment is associated with a negative outcome in subclinical phenotypes of cardiovascular disease and promoting of disease development $(32,35)$. VKA treatment negatively influences atherosclerosis by increasing atherosclerotic plaque vulnerability $(8,36)$. Although it is generally accepted that long term VKA treatment induces vascular calcification, short term treatment of VKA is regarded as safe without detrimental side-effects. Therefore, we tested whether discontinuation of VKA treatment would prevent long term detrimental vascular effects. Discontinuing VKA prevented long term detrimental effects on vascular calcification and atherosclerosis progression. Additional MK7 supplementation did not further attenuate atherosclerosis progression and calcification, likely because of sufficient vitamin K intake in control treated animals (37).

Previous reports have shown that high vitamin K2 intake prevents medial calcification $(17,38,39)$. In our study we specifically focussed on atherosclerotic plaque calcification, which is distinct from medial calcification (40). Intimal calcification is linked to vitamin $\mathrm{K}$ deficiency (8) but also to inflammation and increased oxidative stress $(41,42)$. This corroborates our findings of ucMGP presence in atherosclerotic plaques, highlighting the 
large variation in vitamin K-status in both control and MK7 treated animals. High vitamin K has been shown to have limited effects on ucMGP levels but significantly increases carboxylated MGP levels (43). Indeed, MK7 significantly increased presence of cMGP in atherosclerotic plaques compared to discontinuing of warfarin.

MK7 treated animals displayed reduced levels of oxidative stress in the atherosclerotic plaques. We provide in vitro evidence that warfarin rapidly increased intracellular VSMC oxidative stress and subsequent extracellular vesicle release. It has been shown that both oxidative stress and extracellular vesicle release are features of synthetic VSMCs (44) and involved in atherosclerosis progression and calcification $(42,45,46)$. Cellular oxidative stress is under the control of the master regulator Nrf2, which has been associated with VSMC calcification (47) and Nrf2 activation in VSMC has been shown to have protective effects on atherosclerosis (48). Downstream of Nrf2 is NAD(P)H Quinone Dehydrogenase 1 (NQO1), also termed DT-diaphorase, which is involved in recycling of vitamin K (49). NQO1 is, like vitamin $\mathrm{K}$ epoxide reductase (VKOR), inhibited by VKA. Thus, warfarin could increase oxidative stress via both inhibition of VKOR and NQO1, which could be counteracted by additional vitamin K. Decreased Nrf2 promotes oxidative stress which has been associated with increased cellular senescence (50). Cellular senescence contributes to vascular aging and is associated with elevated inflammation (50) and vascular calcification (51). It has been proposed that vitamin $\mathrm{K}$ is linked to senescence, as a strong inverse correlation was observed between CDKN2A/p16INK4a expression and carboxylated osteocalcin levels. Thus, vitamin $\mathrm{K}$ deficiency may contribute to premature vascular senescence (52).

Oxidative stress is linked to increased extracellular vesicle release, which is a known nidus for vascular calcification $(53,54)$. Warfarin induces a dose-dependent increased secretion of extracellular vesicles, via a vitamin $\mathrm{K}$-dependent mechanism since vitamin $\mathrm{K}$ could abolish secretion of extracellular vesicles. Extracellular vesicles from warfarin treated VSMCs exhibited increased phosphatidylserine (PS) exposure, which is known to promote recognition and clearance via phagocytosis. Extracellular vesicles exposing PS have been observed in human atherosclerotic plaques and contribute to plaque thrombogenicity (55). Gla-residues from gamma-carboxylated vitamin K-dependent proteins can bind to the negatively charged PS $(56,57)$, thereby preventing the formation of a nucleation site. Moreover, calcification and inflammation result in decreased presence of MGP and fetuin-A in extracellular vesicles while enriching PS exposure (46). Fetuin-A is a systemic calcification inhibitor that is internalized and secreted by VSMC derived vesicles $(29,31)$. While fetuin-A is not expressed by VSMC, it is efficiently loaded into VSMC secreted vesicles (31). We injected fluorescently labelled fetuin-A as novel imaging probe. Warfarin treatment resulted in significant uptake of fluorescently labelled fetuin-A in the atherosclerotic plaques. MK7 treated animals had significantly less labelled fetuin-A present in atherosclerotic lesions, suggesting a decreased nidus for calcification. In addition, in vitro MK7 reduced VSMC extracellular vesicle presence. 
In conclusion, discontinuation of short term warfarin use prevents long term detrimental effect on atherosclerosis progression and calcification. MK7 supplementation attenuates atherosclerotic plaque progression by supporting an environment with reduced oxidative stress and increased collagen deposition. These effects of vitamin $\mathrm{K}$ are suggested via controlling VSMC mediated oxidative stress and subsequently could reduce vascular senescence of VSMC.

\section{References}

1. Rennenberg RJMW, Kessels AGH, Schurgers LJ, van Engelshoven JMA, de Leeuw PW, Kroon AA. Vascular calcifications as a marker of increased cardiovascular risk: a meta-analysis. Vasc Health Risk Manag. 2009;5(1):185-97.

2. Budoff MJ, Young R, Burke G, Jeffrey Carr J, Detrano RC, Folsom AR, et al. Ten-year association of coronary artery calcium with atherosclerotic cardiovascular disease (ASCVD) events: the multi-ethnic study of atherosclerosis (MESA). Eur Heart J. 2018 Jul 1;39(25):2401-8.

3. Budoff MJ, Shaw LJ, Liu ST, Weinstein SR, Mosler TP, Tseng PH, et al. Long-term prognosis associated with coronary calcification: observations from a registry of 25,253 patients. J Am Coll Cardiol. 2007 May 8;49(18):1860-70.

4. Raggi P, Callister TQ, Shaw LJ. Progression of coronary artery calcium and risk of first myocardial infarction in patients receiving cholesterol-lowering therapy. Arterioscler Thromb Vasc Biol. American Heart Association, Inc; 2004 Jul;24(7):1272-7.

5. Virmani R, Kolodgie FD, Burke AP, Farb A, Schwartz SM. Lessons from sudden coronary death: a comprehensive morphological classification scheme for atherosclerotic lesions. Arterioscler Thromb Vasc Biol. 2000 May;20(5):1262-75.

6. Joshi NV, Vesey AT, Williams MC, Shah ASV, Calvert PA, Craighead FHM, et al. 18F-fluoride positron emission tomography for identification of ruptured and high-risk coronary atherosclerotic plaques: a prospective clinical trial. Lancet. 2014 Feb 22;383(9918):705-13.

7. van Gorp RH, Schurgers LJ. New Insights into the Pros and Cons of the Clinical Use of Vitamin K Antagonists (VKAs) Versus Direct Oral Anticoagulants (DOACs). Nutrients. 2015;7(11):9538-57.

8. Schurgers LJ, Joosen IA, Laufer EM, Chatrou MLL, Herfs M, Winkens MHM, et al. Vitamin Kantagonists accelerate atherosclerotic calcification and induce a vulnerable plaque phenotype. PLoS ONE. 2012;7(8):e43229.

9. Luo G, Ducy P, McKee MD, Pinero GJ, Loyer E, Behringer RR, et al. Spontaneous calcification of arteries and cartilage in mice lacking matrix GLA protein. Nature. 1997 Mar 6;386(6620):78-81.

10. Murshed M, Schinke T, McKee MD, Karsenty G. Extracellular matrix mineralization is regulated locally; different roles of two gla-containing proteins. J Cell Biol. 2004 Jun 7;165(5):625-30.

11. Schurgers LJ, Teunissen KJF, Knapen MHJ, Kwaijtaal M, van Diest R, Appels A, et al. Novel conformation-specific antibodies against matrix gamma-carboxyglutamic acid (Gla) protein: undercarboxylated matrix Gla protein as marker for vascular calcification. Arterioscler Thromb Vasc Biol. 2005 Aug;25(8):1629-33.

12. Dalmeijer GW, van der Schouw YT, Magdeleyns EJ, Vermeer C, Verschuren WMM, Boer JMA, et al. Circulating desphospho-uncarboxylated matrix $\gamma$-carboxyglutamate protein and the risk of coronary heart disease and stroke. J Thromb Haemost. John Wiley \& Sons, Ltd (10.1111); 2014 Jul;12(7):1028-34.

13. Schurgers LJ, Barreto DV, Barreto FC, Liabeuf S, Renard C, Magdeleyns EJ, et al. The circulating inactive form of matrix gla protein is a surrogate marker for vascular calcification in chronic kidney disease: a preliminary report. Clin J Am Soc Nephrol. American Society of Nephrology; 2010 Apr;5(4):568-75.

14. Ueland T, Dahl CP, Gullestad L, Aakhus S, Broch K, Skårdal R, et al. Circulating levels of nonphosphorylated undercarboxylated matrix Gla protein are associated with disease severity in patients with chronic heart failure. Clin Sci. 2011 Aug;121(3):119-27.

15. Parker BD, Schurgers LJ, Brandenburg VM, Christenson RH, Vermeer C, Ketteler M, et al. The associations of fibroblast growth factor 23 and uncarboxylated matrix Gla protein with mortality in coronary artery disease: the Heart and Soul Study. Ann Intern Med. American College of Physicians; 2010 May 18;152(10):640-8.

16. Wu M, Rementer C, Giachelli CM. Vascular calcification: an update on mechanisms and challenges in treatment. Calcif Tissue Int. Springer US; 2013 Oct;93(4):365-73.

17. Krüger T, Oelenberg S, Kaesler N, Schurgers LJ, van de Sandt AM, Boor P, et al. Warfarin induces cardiovascular damage in mice. Arterioscler Thromb Vasc Biol. 2013 Nov;33(11):2618-24. 
18. Geleijnse JM, Vermeer C, Grobbee DE, Schurgers LJ, Knapen MHJ, van der Meer IM, et al. Dietary intake of menaquinone is associated with a reduced risk of coronary heart disease: the Rotterdam Study. J Nutr. 2004 Nov;134(11):3100-5.

19. Beulens JWJ, Bots ML, Atsma F, Bartelink M-LEL, Prokop M, Geleijnse JM, et al. High dietary menaquinone intake is associated with reduced coronary calcification. Atherosclerosis. 2009 Apr;203(2):489-93.

20. Gast GCM, de Roos NM, Sluijs I, Bots ML, Beulens JWJ, Geleijnse JM, et al. A high menaquinone intake reduces the incidence of coronary heart disease. Nutr Metab Cardiovasc Dis. 2009 Sep;19(7):504-10.

21. Trumbo P, Yates AA, Schlicker S, Poos M. Dietary reference intakes: vitamin A, vitamin K, arsenic, boron, chromium, copper, iodine, iron, manganese, molybdenum, nickel, silicon, vanadium, and zinc. J Am Diet Assoc. 2001 Mar;101(3):294-301.

22. Shea MK, O'Donnell CJ, Hoffmann U, Dallal GE, Dawson-Hughes B, Ordovas JM, et al. Vitamin K supplementation and progression of coronary artery calcium in older men and women. Am J Clin Nutr. 2009 Jun;89(6):1799-807.

23. Brandenburg VM, Reinartz S, Kaesler N, Krüger T, Dirrichs T, Kramann R, et al. Slower Progress of Aortic Valve Calcification With Vitamin K Supplementation. Circulation. 2017 May 23;135(21):2081-3.

24. Westenfeld R, Krueger T, Schlieper G, Cranenburg ECM, Magdeleyns EJ, Heidenreich S, et al. Effect of Vitamin K2 Supplementation on Functional Vitamin K Deficiency in Hemodialysis Patients: A Randomized Trial. YAJKD. Elsevier Inc; 2012 Feb 1;59(2):186-95.

25. Dalmeijer GW, van der Schouw YT, Magdeleyns E, Ahmed N, Vermeer C, Beulens JWJ. The effect of menaquinone-7 supplementation on circulating species of matrix Gla protein. Atherosclerosis. 2012 Dec;225(2):397-402.

26. Wallin R, Martin LF. Warfarin poisoning and vitamin $\mathrm{K}$ antagonism in rat and human liver. Design of a system $<\mathrm{i}>$ in vitro $</ \mathrm{i}>$ that mimics the situation $<\mathrm{i}>\mathrm{in}$ vivo $</ \mathrm{i}>$. Biochem J. Portland Press Limited; 1987 Jan 15;241(2):389-96.

27. Price PA, Faus SA, Williamson MK. Warfarin causes rapid calcification of the elastic lamellae in rat arteries and heart valves. Arterioscler Thromb Vasc Biol. 1998 Sep;18(9):1400-7.

28. Schurgers LJ, Vermeer C. Determination of phylloquinone and menaquinones in food. Effect of food matrix on circulating vitamin K concentrations. Haemostasis. 2000 Oct;30(6):298-307.

29. Kapustin AN, Chatrou MLL, Drozdov I, Zheng Y, Davidson SM, Soong D, et al. Vascular smooth muscle cell calcification is mediated by regulated exosome secretion. Circ Res. Lippincott Williams \& Wilkins; 2015 Apr 10;116(8):1312-23.

30. Terkeltaub RA, Santoro DA, Mandel G, Mandel N. Serum and plasma inhibit neutrophil stimulation by hydroxyapatite crystals. Evidence that serum alpha 2-HS glycoprotein is a potent and specific crystalbound inhibitor. Arthritis Rheum. John Wiley \& Sons, Ltd; 1988 Sep;31(9):1081-9.

31. Reynolds JL, Skepper JN, McNair R, Kasama T, Gupta K, Weissberg PL, et al. Multifunctional roles for serum protein fetuin-a in inhibition of human vascular smooth muscle cell calcification. Journal of the American Society of Nephrology. American Society of Nephrology; 2005 Oct;16(10):2920-30.

32. Weijs B, Blaauw Y, Rennenberg RJMW, Schurgers LJ, Timmermans CCMM, Pison L, et al. Patients using vitamin $\mathrm{K}$ antagonists show increased levels of coronary calcification: an observational study in low-risk atrial fibrillation patients. Eur Heart J. 2011 Oct;32(20):2555-62.

33. Peeters F, Dudink E, Kimenai D, Weijs B, Altintas S, Heckman L, et al. Vitamin K Antagonists, NonVitamin K Antagonist Oral Anticoagulants, and Vascular Calcification in Patients with Atrial Fibrillation. TH Open. Georg Thieme Verlag KG; 2018 Oct 1;02(04):e391-8.

34. Mujaj B, Bos D, Muka T, Lugt AVD, Ikram MA, Vernooij MW, et al. Antithrombotic treatment is associated with intraplaque haemorrhage in the atherosclerotic carotid artery: a cross-sectional analysis of The Rotterdam Study. Eur Heart J. 2018 Jul 27.

35. Eggebrecht L, Prochaska JH, Schulz A, Arnold N, Jünger C, Göbel S, et al. Intake of Vitamin K Antagonists and Worsening of Cardiac and Vascular Disease: Results From the Population-Based Gutenberg Health Study. J Am Heart Assoc. 2018 Sep 4;7(17):1396-25.

36. Plank F, Beyer C, Friedrich G, Stühlinger M, Hintringer F, Dichtl W, et al. Influence of vitamin K antagonists and direct oral anticoagulation on coronary artery disease: A CTA analysis. International Journal of Cardiology. Elsevier B.V; 2018 Jun 1;260(C):11-5.

37. Harshman SG, Fu X, Karl JP, Barger K, Lamon-Fava S, Kuliopulos A, et al. Tissue Concentrations of Vitamin K and Expression of Key Enzymes of Vitamin K Metabolism Are Influenced by Sex and Diet but Not Housing in C57B16 Mice. J Nutr. 2016 Aug;146(8):1521-7.

38. Schurgers LJ, Teunissen KJF, Hamulyák K, Knapen MHJ, Vik H, Vermeer C. Vitamin K-containing dietary supplements: comparison of synthetic vitamin K1 and natto-derived menaquinone-7. Blood. 2007 Apr 15;109(8):3279-83. 
39. McCabe KM, Booth SL, Fu X, Shobeiri N, Pang JJ, Adams MA, et al. Dietary vitamin K and therapeutic warfarin alter the susceptibility to vascular calcification in experimental chronic kidney disease. Kidney Int. 2013 May;83(5):835-44.

40. Amann K. Media calcification and intima calcification are distinct entities in chronic kidney disease. Clin J Am Soc Nephrol. American Society of Nephrology; 2008 Nov;3(6):1599-605.

41. Shanahan CM. Inflammation ushers in calcification: a cycle of damage and protection? Circulation. Lippincott Williams \& Wilkins; 2007 Dec 11;116(24):2782-5.

42. Byon $\mathrm{CH}$, Chen Y. Molecular Mechanisms of Vascular Calcification in Chronic Kidney Disease: The Link between Bone and the Vasculature. Curr Osteoporos Rep. 2015 May 7.

43. Schurgers LJ, Spronk HMH, Soute BAM, Schiffers PM, DeMey JGR, Vermeer C. Regression of warfarininduced medial elastocalcinosis by high intake of vitamin K in rats. Blood. 2007 Apr 1;109(7):2823-31.

44. Branchetti E, Poggio P, Sainger R, Shang E, Grau JB, Jackson BM, et al. Oxidative stress modulates vascular smooth muscle cell phenotype via CTGF in thoracic aortic aneurysm. Cardiovasc Res. 2013 Nov $1 ; 100(2): 316-24$.

45. Byon CH, Javed A, Dai Q, Kappes JC, Clemens TL, Darley-Usmar VM, et al. Oxidative stress induces vascular calcification through modulation of the osteogenic transcription factor Runx 2 by AKT signaling. J Biol Chem. 2008 May 30;283(22):15319-27.

46. Kapustin AN, Shanahan CM. Emerging roles for vascular smooth muscle cell exosomes in calcification and coagulation. J Physiol (Lond). 2016 Jun 1;594(11):2905-14.

47. Wei R, Enaka M, Muragaki Y. Activation of KEAP1/NRF2/P62 signaling alleviates high phosphateinduced calcification of vascular smooth muscle cells by suppressing reactive oxygen species production. Sci Rep. Nature Publishing Group; 2019 Jul 17;9(1):10366-13.

48. Bozaykut P, Karademir B, Yazgan B, Sozen E, Siow RCM, Mann GE, et al. Effects of vitamin E on peroxisome proliferator-activated receptor $\gamma$ and nuclear factor-erythroid 2-related factor 2 in hypercholesterolemia-induced atherosclerosis. Free Radical Biology and Medicine. 2014 May;70:17481.

49. Ingram BO, Turbyfill JL, Bledsoe PJ, Jaiswal AK, Stafford DW. Assessment of the contribution of $\mathrm{NAD}(\mathrm{P}) \mathrm{H}$-dependent quinone oxidoreductase 1 (NQO1) to the reduction of vitamin K in wild-type and NQO1-deficient mice. Biochem J. 2013 Nov 15;456(1):47-54.

50. Ungvari Z, Tarantini S, Nyúl-Tóth Á, Kiss T, Yabluchanskiy A, Csipo T, et al. Nrf2 dysfunction and impaired cellular resilience to oxidative stressors in the aged vasculature: from increased cellular senescence to the pathogenesis of age-related vascular diseases. Geroscience. Springer International Publishing; 2019 Oct 26;133(5):446-12.

51. Ragnauth CD, Warren DT, Liu Y, McNair R, Tajsic T, Figg N, et al. Prelamin A acts to accelerate smooth muscle cell senescence and is a novel biomarker of human vascular aging. Circulation. Lippincott Williams \& Wilkins; 2010 May 25;121(20):2200-10.

52. Stenvinkel P, Luttropp K, McGuinness D, Witasp A, Qureshi AR, Wernerson A, et al. CDKN2A/p16INK4a expression is associated with vascular progeria in chronic kidney disease. Aging (Albany NY). 2017 Feb 9;9(2):494-507.

53. Kapustin AN, Davies JD, Reynolds JL, McNair R, Jones GT, Sidibe A, et al. Calcium regulates key components of vascular smooth muscle cell-derived matrix vesicles to enhance mineralization. Circ Res. 2011 Jun 24;109(1):e1-12.

54. Goettsch C, Hutcheson JD, Aikawa M, Iwata H, Pham T, Nykjaer A, et al. Sortilin mediates vascular calcification via its recruitment into extracellular vesicles. J Clin Invest. 2016 Mar 7.

55. Mallat Z, Hugel B, Ohan J, Lesèche G, Freyssinet JM, Tedgui A. Shed membrane microparticles with procoagulant potential in human atherosclerotic plaques: a role for apoptosis in plaque thrombogenicity. Circulation. 1999 Jan 26;99(3):348-53.

56. Kapustin AN, Schoppet M, Schurgers LJ, Reynolds JL, McNair R, Heiss A, et al. Prothrombin Loading of Vascular Smooth Muscle Cell-Derived Exosomes Regulates Coagulation and CalcificationHighlights. Arterioscler Thromb Vasc Biol. American Heart Association, Inc; 2017 Mar 1;37(3):e22-e32.

57. Schurgers LJ, Spronk HMH, Skepper JN, Hackeng TM, Shanahan CM, Vermeer C, et al. Posttranslational modifications regulate matrix Gla protein function: importance for inhibition of vascular smooth muscle cell calcification. J Thromb Haemost. 2007 Dec;5(12):2503-11. 
VITAMIN K METABOLISM IN ATHEROSCLEROSIS 


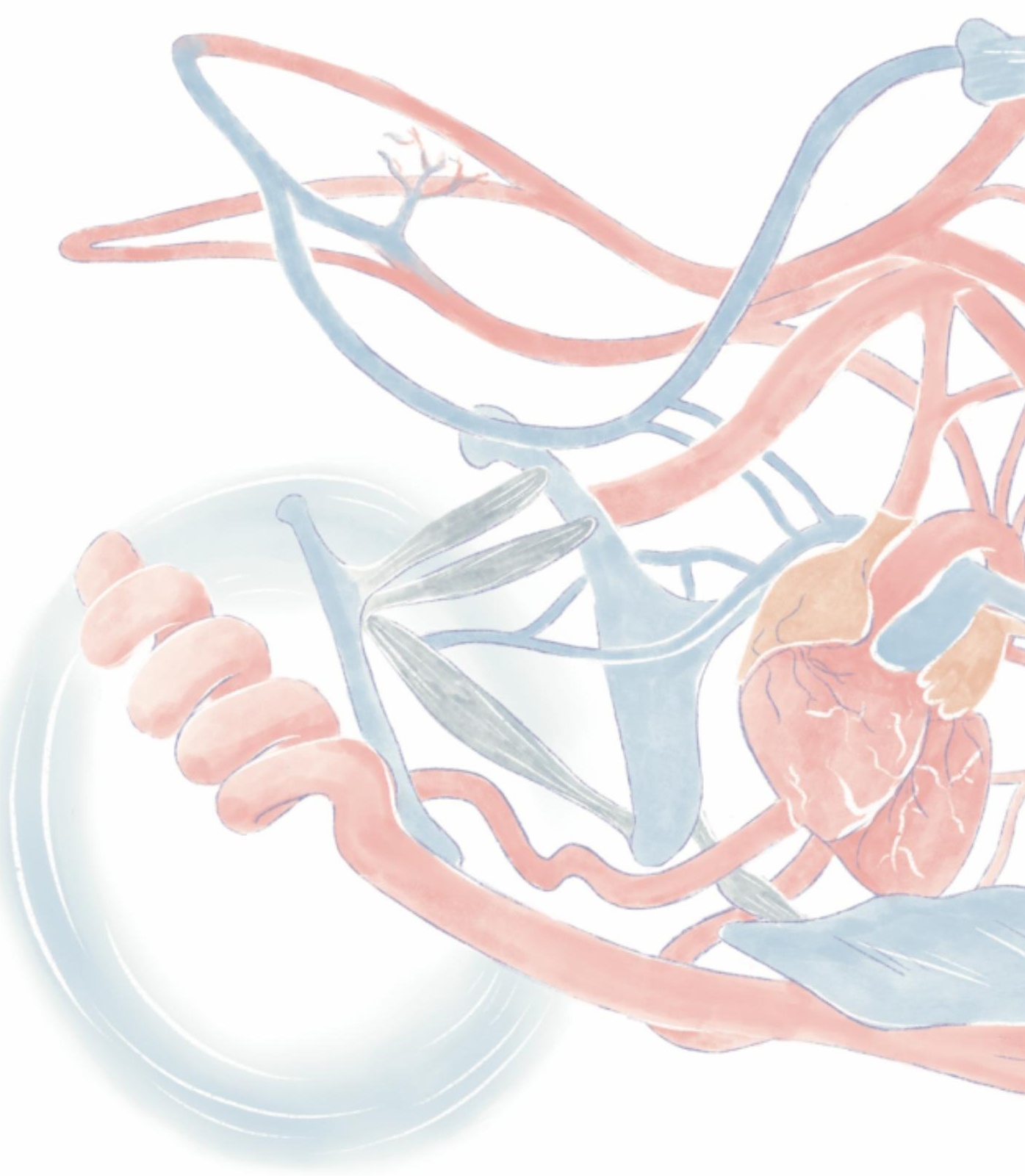




\section{Chapter 5}

\section{Off-target effects of oral anticoagulants - Vascular effects of vitamin $K$ antagonist and non-vitamin $K$ antagonist oral anticoagulants}

RH van Gorp, I Dijkgraaf, M Bauwens, P Leenders, D Jennen, MR

Dweck, J Bucerius, JJ Briedé, J van Ryn, V Brandenburg, F Mottaghy, HMH Spronk, CP Reutelingsperger, LJ Schurgers. 


\begin{abstract}
Introduction: Vitamin K Antagonists (VKA) and direct non-vitamin K antagonists (NOAC) are used in the clinic to reduce the risk of thrombosis. However, both oral anticoagulant agents alter non-haemostatic effects in the arterial vasculature as well. VKA treatment is associated with increased vascular calcification in both preclinical and clinical studies. Initial studies with NOAC point towards beneficial inhibitory effects on atherogenesis. Here, we investigated both short- and long-term effects of VKA and the NOAC, dabigatran on vascular calcification and atherosclerosis development.
\end{abstract}

Material and methods: Female $A p o e^{-/}$mice (age 12 weeks) were fed western type diet (WTD) as control $(\mathrm{n}=24)$ or supplemented with dabigatran etexilate (dabigatran; $7.5 \mathrm{mg} / \mathrm{g}$; $\mathrm{n}=24)$ or warfarin $(3 \mathrm{mg} / \mathrm{g} ; \mathrm{n}=24)$ for 6 or 18 weeks. Vascular calcification was measured in whole aortic arches using $\mu \mathrm{CT}$ and ${ }^{18} \mathrm{~F}-\mathrm{NaF}$. Atherosclerotic burden in aortic arches was assessed by haematoxyline \& eosin histochemistry followed by determination of plaque parameters using (immuno)histochemistry. Additionally, in vitro effects of warfarin, thrombin and dabigatran on primary vascular smooth muscle cells (VSMC) were assessed.

Results: Short-term treatment with warfarin promoted formation of atherosclerotic lesions with a pro-inflammatory phenotype, and more rapid plaque progression compared to control and dabigatran. In contrast, dabigatran significantly reduced plaque progression compared to control. Long-term warfarin treatment significantly increased both activity and presence of plaque calcification compared to control and dabigatran. Dabigatran treatment showed a nonsignificant reduction in calcification as detected by $\mu \mathrm{CT}$ compared to control. Calcification induced by warfarin treatment was accompanied by increased presence of uncarboxylated matrix Gla protein and bone morphogenetic protein 4. In vitro, both warfarin and thrombin significantly increased VSMC oxidative stress and extracellular vesicle release, which was prevented by dabigatran.

Conclusion: Warfarin aggravates atherosclerotic disease activity, increasing plaque inflammation, active calcification and plaque progression. In contrast, dabigatran shows beneficial effects on atherosclerosis progression and lacks undesired side effects on vascular calcification. The choice of anticoagulation impacts atherosclerotic disease by differential off target effect. 


\section{Introduction}

Upon atherosclerotic plaque rupture or erosion, atherothrombosis may cause the occlusion of arteries which remains the main cause of morbidity and mortality worldwide (1, 2). For decades, vitamin K-antagonists (VKAs) have been the most widely used anticoagulant drugs for treatment of patients at risk of arterial and venous thrombosis. Due to unfavorable pharmacokinetics of VKA, direct thrombin and factor Xa (FXa) inhibitors (direct non-vitamin $\mathrm{K}$ antagonist oral anticoagulants, NOACs) have been introduced to the clinic as alternative anticoagulants.

Both VKA and NOACs are proven effective in reducing thrombotic risk, however they also influence non-hemostatic activities of coagulation factors. VKAs exhibits its side effects on the vessel wall via vitamin $\mathrm{K}$ dependent proteins (VKDP) $(3,4)$. In the vasculature, vascular smooth muscle cells (VSMC) synthesize the vitamin K dependent matrix Gla protein (MGP), which acts as a local vascular calcification inhibitor (5). Inhibition of MGP activity by VKA treatment is associated with increased vascular calcification (6). VKA treatment increases intimal calcification in patients and experimental animal models of atherosclerosis $(7,8)$. The consequences of vascular calcification on plaque stability are debated and dependent on the extend of calcification, size, localization and density (9). Macrocalcification, or sheet-like calcification, and dense calcification are suggested to stabilize the atherosclerotic plaque (10). In contrast, microcalcification, or spotty calcification, and increased calcification volume have been implicated in promoting plaque instability, and subsequently increasing risk of plaque rupture and risk for stroke and myocardial infarction (11-13). Microcalcifications are metabolically active and can be detected with the PET tracer ${ }^{18} \mathrm{~F}-\mathrm{NaF}$ (14). Recently, it was shown that VKA use was associated with increased spotty coronary artery calcification (15).

The NOAC dabigatran etexilate (DE) was one of the first non-vitamin $\mathrm{K}$ oral antagonist on the market. DE is an oral prodrug that is rapidly converted by esterases to dabigatran, which is a direct, competitive inhibitor of thrombin (factor IIa). Thrombin is a key enzyme in the coagulation cascade, ensuring the conversion of fibrinogen into fibrin. Thrombin has also a wide spectrum of effects directly on the vessel wall, which contribute to atherogenesis $(16,17)$. Increased thrombin formation has been shown to aggravate atherogenesis (18). Inhibition of thrombin by dabigatran reduced plaque size and atherogenesis $(18,19)$. Since dabigatran exhibits these beneficial effects on reducing plaque size, we addressed the question whether dabigatran also has an effect on plaque calcification. Using a pre-clinical experimental animal model of atherosclerosis, we assessed the shortand the long-term effect of the VKA warfarin and the NOAC dabigatran on atherosclerotic plaque calcification and atherogenesis.

\section{Materials and methods}


Experimental animals. All animal studies were performed under an approved protocol by the Ethics Committee for animal experiments of Maastricht University. Twelve-week-old female C57/BL6 Apoe -/- $^{-}$mice were purchased from Maastricht University and housed in climate-controlled spaces under 12-hour day/night cycle with ad libitum access to food and water. Mice were fed an irradiated (0.9Mrad) vitamin K-deficient Western Type Diet (WTD: $0.25 \%$ cholesterol and $15 \%$ cocoa butter, derived from Altromin, Lage, Germany). WTD was supplemented with in corn oil dissolved vitamin K1 (5 mg/Kg, Merck KGaA, Darmstadt, Germany) and was used as control diet or supplemented with dabigatran etexilate (DE; 7.5 $\mathrm{mg} / \mathrm{g}$, Boehringer Ingelheim, Ingelheim, Germany) for NOAC treatment. Additionally, WTD was supplemented with warfarin (3 mg/g warfarin; Merck KGaA, Darmstadt, Germany and vitamin K1 (1.5 mg/g K1, Merck KGaA, Darmstadt, Germany), for VKA treatment. Vitamin $\mathrm{K} 1$, which counteracts the warfarin's effects predominantly in liver and less in extrahepatic tissue, was additionally added to the warfarin diet to prevent bleeding $(8,20)$.

Mice were randomly divided to receive WTD (control), DE (dabigatran) or warfarin (warfarin) supplemented food for 6 or 18 weeks ( $n=12$ per treatment per time point; Figure 1A). Hereafter, mice were sacrificed, and blood was collected in $105 \mathrm{mM}$ trisodium citrate via the portal vein. Plasma was prepared and frozen in aliquots at $-80^{\circ} \mathrm{C}$ until analysis. Next, the vasculature was washed with a sterile binding buffer (40 mM Hepes, $150 \mathrm{mM} \mathrm{NaCl}, 5$ $\mathrm{mM} \mathrm{KCl}, 1 \mathrm{mM} \mathrm{MgCl} 2$ and $2.5 \mathrm{mM} \mathrm{CaCl}_{2}, \mathrm{pH} \mathrm{7.3)}$ via the left ventricle. Aortas were harvested, dissected and fixed in 1\% (v/v) HEPES-buffered formaldehyde containing 150 $\mathrm{mM}$ saline, overnight at $4^{\circ} \mathrm{C}$, before they were embedded in paraffin. Before sections were cut, paraffin embedded aortas were analyzed ex vivo for calcification using $\mu \mathrm{CT}$.

Blood analysis. Warfarin and dabigatran plasma levels were determined using HPLC and Hemoclot thrombin inhibitor assay (HTI; Hyphen Biomed, Neuville-sur-Oise, France), respectively. The prothrombin time of plasma, a measure for clotting tendency, was determined according to standard laboratory methods using the appropriate tests. International normalized ratio (INR) was calculated using the prothrombin time of untreated mice (control mice) as baseline. Total plasma cholesterol and triglyceride levels were assessed with the Synchron LX20 (Beckman Coulter, Brea, USA) according to manufacturer's instructions.

${ }^{18} \mathbf{F}-\mathrm{NaF}$. Mice ( $\mathrm{n}=7,6$ and 8 for control, warfarin and DE, respectively) were fasted for two hours before intravenously injection with ${ }^{18} \mathrm{~F}-\mathrm{NaF}$. Thirty minutes post injection, the animals were killed by an overdose of pentobarbital and the vasculature was washed with a sterile binding buffer ( $40 \mathrm{mM}$ Hepes, $150 \mathrm{mM} \mathrm{NaCl}, 5 \mathrm{mM} \mathrm{KCl}, 1 \mathrm{mM} \mathrm{MgCl} 2$ and $2.5 \mathrm{mM} \mathrm{CaCl}_{2}$, $\mathrm{pH} 7.3$ ) via the left ventricle. Aortic arch was dissected and ${ }^{18} \mathrm{~F}-\mathrm{NaF}$ uptake was measured ex vivo using autoradiography and with a gamma counter (Wallac Wizard, Turku, Finland). Uptake was corrected for injected dose and time.

$\boldsymbol{\mu C T}$ analysis of aortic calcification. Ex vivo $\mu \mathrm{CT}$ scans were performed to quantify calcification in the whole aortic tissue ( $n=11$ for control and DE, and $n=12$ for warfarin treatment). The paraffin embedded aortas were scanned for 40/50 minutes by $\mu \mathrm{CT}$ 
(NanoSPECT/CT ${ }^{\circledR}$, Bioscan, USA) using $45 \mathrm{keV}$ energy, and 360 projections with a resolution of $0.1 \mathrm{~mm}$. Scan data were assessed for Hounsfield units (HU), with $\mathrm{HU}>1000$ considered as positive calcification.

Immunohistochemistry. Embedded aortic sections were cut at $4 \mu \mathrm{m}$ thickness. Plaque size and volume was determined using hematoxyline and eosine $(\mathrm{H} / \mathrm{E})$ staining. Additionally, plaque characteristics were determined using histochemical analysis for calcification (Alizarin Red S), and elastin fiber breaks (Elastica von Giesson). Stained sections were analyzed by ImageJ Software. Elastin breaks found beneath the atherosclerotic plaque were scored in different sections for every mouse, using a scoring system ranging from 0 to 4 ; 0 , no breaks; 1, one-three breaks; 2, four-six breaks; 3, seven-nine breaks and 4, > 9 breaks.

The following antibodies were used: anti-uncarboxylated matrix Gla-protein (1:400; IDS, Boldon, UK), anti-BMP4 (1:50; Santa Cruz Biotechnology, Dallas, TX, USA), anti-8hydroxyguanisine (8OHdG; 1:300; Meridian Life Science, Tennessee, USA), anti-MAC3 (1:50, Bioscience, Merck KGaA, Germany) and anti-integrin ß 3 (ITGB3 1:100, SigmaAldrich). HRP conjugated secondary antibodies were visualized with Nova-RED substrate (Vector Labs, Amsterdam, Netherlands). 2D analysis quantification was performed using Leica Application Suite X (Leica Microsystems, Wetzlar, Germany). Data were expressed as the percentage of positive plaque area unless otherwise stated.

Cell culture. Human primary VSMC were derived from tissue explants from males and females aged between 18 and 65 years of age and cultured as described previously (21). In brief, VSMC were cultured in DMEM medium (Gibco, Thermo Fisher Scientific Inc, Paisley, Scotland, UK) supplemented with 20\% fetal bovine serum (FBS), $100 \mathrm{U} / \mathrm{mL}$ penicillin and $100 \mu \mathrm{g} / \mathrm{mL}$ streptomycin. Cells were used between passage 4 and 10 .

Calcification assays. VSMC were placed in calcifying medium consisting of DMEM supplemented with $2.5 \% \mathrm{FBS}$ and $1 \%$ PS in the presence of an additional $1.8 \mathrm{mmol} / \mathrm{L}$ calcium. After incubation with stimulus calcium deposits were assessed by the $\mathrm{O}$ cresolphthalein method and calcium content was normalized for protein content.

VSMC proliferation. $x$ CELLigence, VSMC were seeded in 96-xCELLigence-well. After 24 hours stimulus was added directly in the well. The slope of impudence over 3 days was determined as an indication of proliferation. $E d U$, VSMC were seeded in a 24 well-plate. The cells were allowed to adhere overnight, then the medium was replaced with stimulus and EdU (10 $\mu \mathrm{M}$, Life Technologies) containing 0.5\% FBS. After 24 hours, cells were washed twice and fixed using 4\% paraformaldehyde (Merck KGaA, Darmstadt, Germany). Cells were then incubated with staining solution ( $0.1 \mathrm{M}$ Tris, $1 \mathrm{mM} \mathrm{CuSO} 4,10.5 \mu \mathrm{M}$ azide-F488 (click chemistry tools) and $0.1 \mathrm{M}$ ascorbic acid) for one hour in the dark. Cells were counterstained with Hoechst $(1 \mu \mathrm{g} / \mathrm{mL})$ for $15 \mathrm{~min}$. GFP and DAPI images were obtained using Cytation 3 Cell Imaging Multi-Mode Reader (BioTek Instruments Inc., Winooski, VT, USA) and percentage of GFP positive cells over all cells were calculated.

VSMC migration. VSMC migration was determined using xCELLigence using Boyden chamber principal. First, VSMC were starved overnight in $0.5 \%$ FBS. Next day, bottom and 
upper chamber were coated with collagen $(0.1 \mathrm{mg} / \mathrm{mL}$ collagen $\mathrm{G}$, Biochrome MERKC). Hereafter, 40.000 VSMC were placed in the upper chamber and migration was analyzed over the following 4 hours.

qPCR. RNA extraction, cDNA synthesis and qPCR were performed as described previously (22). The following primers were used: MCP1 forward; 5'-CCCCAGTCACCTGCTGTTAT -3, MCP1 reverse; 5'-TGGAATCCTGAACCCACTTC -3, IFN $\gamma$ forward; 5'CCAACGCAAAGCAATACATGA $-3, \quad$ IFN $\gamma \quad \gamma \quad$ reverse; 5'CCTTTTTCGCTTCCCTGTTTTA -3, IL1 ß forward; 5'-AAACCTCTTCGAGGCACAAG -3, IL1 ß reverse; 5'- GTTTAGGGCCATCAGCTTCA -3, TNF $\alpha$ forward; 5'TGCACTTTGGAGTGATCGGC $\quad-3, \quad$ TNF $\alpha \quad$ reverse; AGCTTGAGGGTTTGCTACAACA -3, 5'--3, and for normalization: GAPDH forward; 5'AACGGATTTGGTCGTATTGGGC-3, GAPDH reverse; CTTGACGGTGCCATGGAATTTG-3.

mRNA isolation for micro-assay. VSMC were fasted overnight and stimulated with thrombin $(1 \mathrm{U} / \mathrm{mL})$, warfarin $(50 \mu \mathrm{M})$ or control for 4 hours. After 4 hours, VSMC were washed in ice cold PBS before RNA was isolated using the miRNeasy Mini Kit (Qiagen, The Netherlands) including a DNase treatment, according to the manufacturer's protocols. The concentration of total RNA was measured on a Nanodrop ${ }^{\circledR}$ ND-1000 spectrophotometer (Thermofischer, The Netherlands). The integrity of total RNA was checked using RNA Nanochips on a 2100 Bioanalyzer (Agilent Technologies, The Netherlands). Only samples with an RNA Integrity Number (RIN) higher than 8.9 were used for microarray analysis.

Microarray preparation and data validation. A total amount of $200 \mu \mathrm{g}$ RNA of each sample was used as input material for synthesizing dye (Cyanine 3 or Cy3) labeled complimentary-RNA according to the Agilent one-color Quick-Amp labeling protocol (Agilent Technologies, Amstelveen, The Netherlands). Individual samples were hybridized to an Agilent SurePrint G3 Human Gene Expression $8 \times 60 \mathrm{~K}$ v2 (Agilent Technologies, Santa Clara, CA, USA). The microarrays were scanned using the Agilent Microarray Scanner (AgilentTechnologies, Amstelveen, The Netherlands). Pixel intensities were extracted as raw data from the scan images using Agilent Feature Extraction Software (Agilent). Quantile normalization and data processing were performed using ArrayQC (https://github.com/BiGCAT-UM/arrayQC Module/), a quality control pipeline in $\mathrm{R}$ (version 2.10.1; The R Foundation for Statistical Computing, Vienna, Austria). For each spot the following steps were taken: local background correction, flagging of bad spots, controls and spots with too low intensity, $\log 2$ transformation, and quantile normalization. Probes with no flagged bad spots were selected and repeated identifiers merged, resulting in 21532 transcripts, representing 15163 unique known genes, were used for statistical analysis.

Selection of differentially expressed genes. Differentially expressed genes (DEGs) were selected using the linear model for microarrays (LIMMA) approach (23). The resulting $p$ values were FWER-corrected using the False Discovery Rate (FDR) method. The following criteria were applied: (1) a FDR-corrected $p$-value $<0.05$ obtained through a moderated $t$ - 
test, and (2) for the biological replicates an average absolute fold change (FC) of 1.5 or higher. Selected gene data sets used "inflammation" and "atherosclerosis" are available on disgenet.

Intracellular generation of oxidative stress. To identify intracellular ROS, we used the cell permeable fluorogenic substrate 2,7 - dichlorofluorescein diacetate (DCFDA, Merck KGaA, Darmstadt, Germany) which is oxidized to 2,7 -dichlorofluorescein in the presence of oxidants.

VSMC were seeded in a 96-well plate and left to adhere overnight. Next, cells were incubated with $20 \mu \mathrm{M}$ DCFDA for $60 \mathrm{~min}$ in the dark at $37^{\circ} \mathrm{C}$ and $5 \% \mathrm{CO}$. After washing, cells were incubated with stimulus in combination with Hoechst $(1 \mu \mathrm{g} / \mathrm{mL})$ and fluorescence intensity was measured (Excitation 485, Emission 529) with Cytation 3 Cell Imaging Multi-Mode Reader (BioTek Instruments Inc., Winooski, VT, USA) for a total of 30 minutes. Fluorescence intensity was corrected for background measurement and normalized to cell count.

Extracellular vesicles quantification. VSMC were seeded in a 12-well plate and allowed to adhere overnight. Next, VSMC were stimulated in DMEM 31966, supplemented with $0.5 \%$ FBS and $1 \%$ PS for 24 hours, after which medium was collected. Medium was centrifuged at $3000 \mathrm{x}$ g and incubated with CD-63 coupled beads overnight. After washing with PBS-2\%BSA, beads were incubated with secondary antibody CD81-APC (1:50; BD biosciences) and incubated for 60 minutes in the dark. After washing, exosomes were detected by flowcytometry (BD Accuri C6). Exosome secretion is expressed as arbitrary units, which were calculated as follows: median fluorescence was multiplied by percentage positive beads and this was normalized for cell number.

Statistical analysis. All in vitro data were obtained in three or more independent experiments in triplicates (or more) wells. Data are expressed as mean with standard deviation (SD). Data were analyzed using the Mann-Whitney T-test or Kruskal-Wallis One-way ANOVA using PRISM software (Graphpad 8.2.1, San Diego, US). Values of $\mathrm{p}<0.05$ were considered statistically significant.

\section{Results}

Female B16/C57-Apoe ${ }^{-/-}$mice were fed a western type diet (WTD; control) or WTD supplemented with VKA (Warfarin) or dabigatran etexilate (DE; dabigatran) for 6 or 18 weeks (Figure 1A). No difference was observed in weight, cholesterol and triglycerides levels between the groups after 18 weeks of treatment (Table 1). Increased warfarin and dabigatran plasma levels confirmed respective treatment. Moreover, warfarin significantly increased prothrombin time compared to control, confirming anticoagulant effect (Table 1).

Effect of short-term oral anticoagulation on atherosclerotic development.

Table 1. Model validation of control, warfarin and dabigatran treatment. 


\begin{tabular}{|c|c|c|c|}
\hline Weight (gram) & $23.82 \pm 1.60$ & $22.50 \pm 1.68$ & $24.33 \pm 2.46$ \\
\hline Cholesterol (mM) & $21.27 \pm 3.34$ & $21.54 \pm 3.07$ & $18.93 \pm 1.74$ \\
\hline Triglycerides $(\mathbf{m M})$ & $2.77 \pm 0.20$ & $2.96 \pm 0.28$ & $2.70 \pm 0.17$ \\
\hline Dabigatran $(\mathbf{n g} / \mathbf{m L})$ & - & - & $370.3 \pm 175.9$ \\
\hline Warfarin $(\mathbf{m g} / \mathbf{L})$ & - & $11,52 \pm 5,81$ & - \\
\hline Prothrombin time $(\mathbf{s e c})$ & $8.73 \pm 0.83$ & $54.2 \pm 22.7^{*}$ & $12.5 \pm 4.28$ \\
\hline
\end{tabular}

$* \mathrm{p}<0.05$

Serial sections of the aortic arch were used to visualize atherosclerotic plaques. Firstly, we compared two methods to express plaque size: 1) plaque size was based on the section with the largest measured plaque area in $\mathrm{mm}^{2}$, or 2) plaque size was based on volume $\left(\mathrm{mm}^{3}\right)$ determined by reconstructing every $5^{\text {th }}$ section of serially cut specimen. The two methodsshowed a very strong correlation ( $\mathrm{r} 0.911, \mathrm{p}<0.0001$; supplemental Figure 1A), thus due time consumption we selected the first method and expressed plaque size in $\mathrm{mm}^{2}$. Shortterm treatment did not significantly alter plaque size between groups, although warfarin showed a trend towards smaller plaques compared to control and dabigatran treatment $(\mathrm{p}=$ 0.24 and $p=0.09$ for warfarin vs control and dabigatran, respectively; Figure 1B). Next, we measured the inflammatory status of plaques by staining macrophage and macrophage-like cells using anti-MAC3 antibodies. Warfarin treatment significantly increased MAC3 positive staining (38.9\% of plaque area) as compared to both control $(25.8 \%$ of plaque area) and dabigatran treatment (23.9\% of plaque area) (Figure 1C). At 6 weeks of treatment, Alizarin Red S stain did not detect any calcification in any of the groups (data not shown). However, warfarin treatment significantly increased uncarboxylated MGP (ucMGP) in atherosclerotic lesions as compared to both control and dabigatran ( $p<0.05$; Figure 1D). Furthermore, a trend of increased integrin $\beta 3$ (ITGß3) expression was observed after warfarin treatment

A

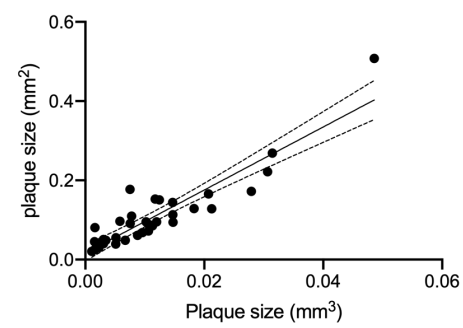

B

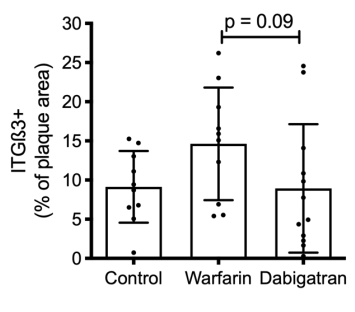

C

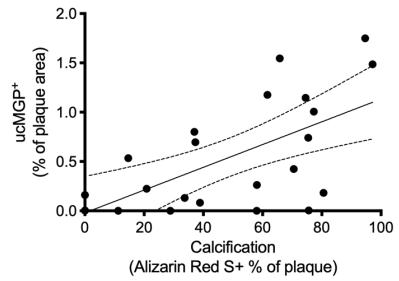

Supplemental Figure 1. Atherosclerotic plaque determination. A) Plaque size based on the section with the largest measured plaque area in $\mathrm{mm}^{2}$ or based on volume $\left(\mathrm{mm}^{3}\right)$ determined by reconstructing every $5^{\text {th }}$ section of serially cutted specimen showed a very strong significant correlation ( $\mathrm{r} 0.911, \mathrm{p}<0.0001)$. B) Short-term treatment with warfarin resulted in a trend towards increased integrinß3 (ITGß3) presence in atherosclerotic plaques compared to dabigatran treatment. C) UcMGP detected in atherosclerotic plaques correlates strongly and significantly with presence of vascular calcification ( $\mathrm{r} 0.58, \mathrm{p}<0.01)$. 

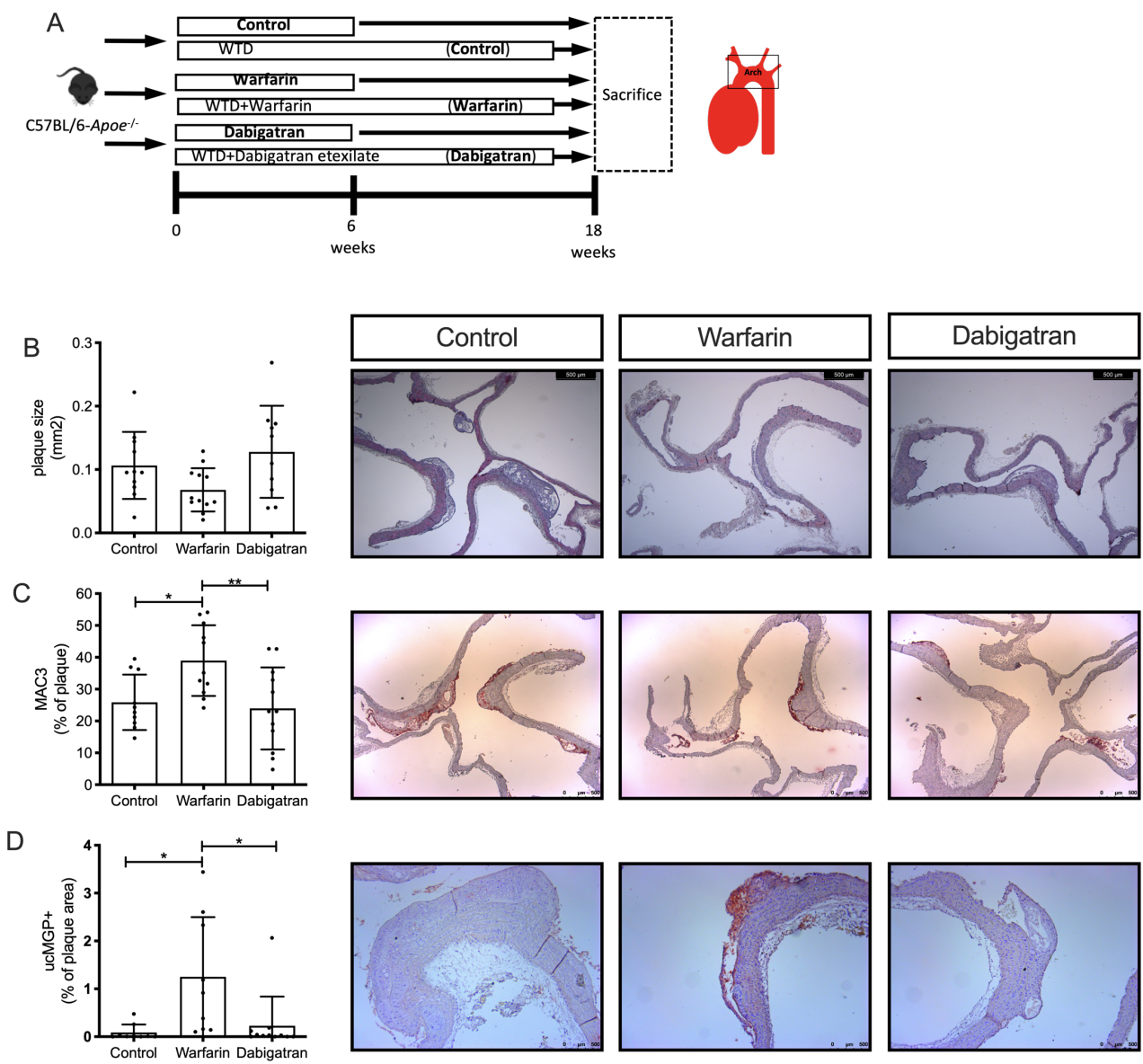

Figure 1. Short-term oral anticoagulant effects on atherosclerotic lesions. A) Schematic overview of experimental set-up. B) Aortic arches of Apoe $^{-/-}$mice on WTD without or with warfarin or dabigatran showed no significant difference on plaque size. C) MAC-3+ cells were significantly increased in lesions of warfarin treated mice compared to control $(\mathrm{p}<0.05)$ and dabigatran $(\mathrm{p}<0.01)$. D) Warfarin treatment significantly increased ucMGP in atherosclerosis lesions compared to control and dabigatran.

compared to dabigatran ( $\mathrm{p}=0.09$; Supplemental Figure 1B). No significant difference was observed in smooth muscle cell content ( $\alpha$ SMA) and oxidative stress levels (8-OhdG) between the plaques of the groups (data not shown).

Long-term VKA treatment increases active vascular calcification.

We next determined long-term effects of warfarin treatment on plaque progression and calcification and compared those with control and dabigatran treatment. Firstly, the amount of vascular calcification was detected by $\mu \mathrm{CT}$ in whole mouse aortic arches. Warfarin treatment significantly increased vascular calcification compared to control $(30.8 \pm 31.9$ and $6.22 \pm 4.59$ total hounsfield units for warfarin and control, respectively; $p<0.05)$ and 


\section{Chapter 5}

A

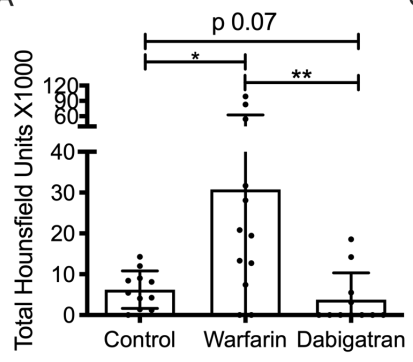

B

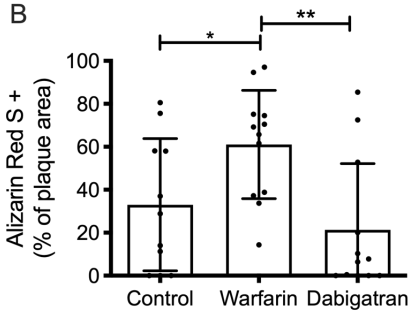

D

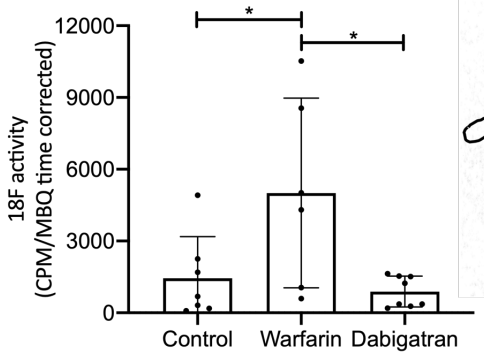

G

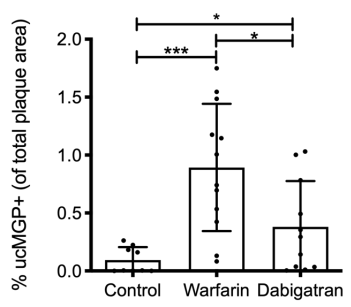

$\mathrm{H}$

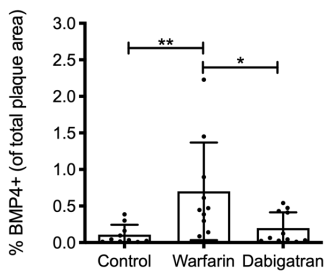

C

E
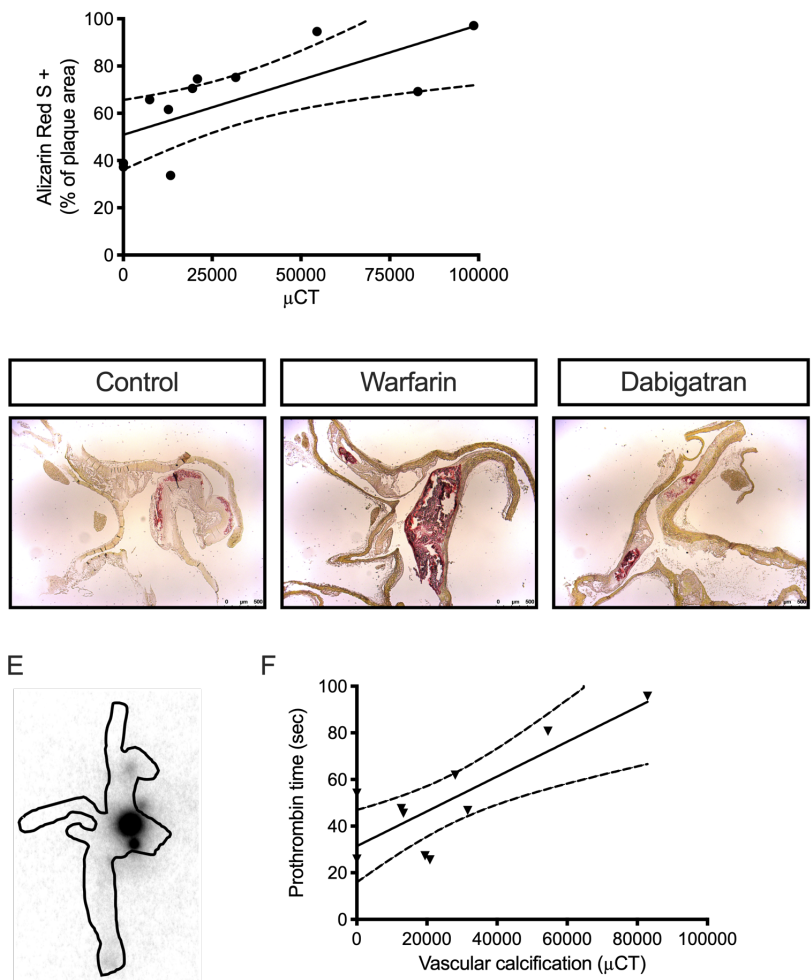

$\mathrm{F}$

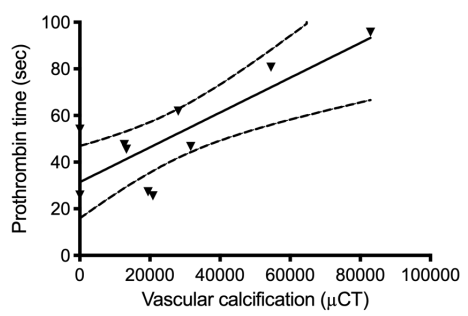

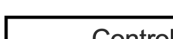
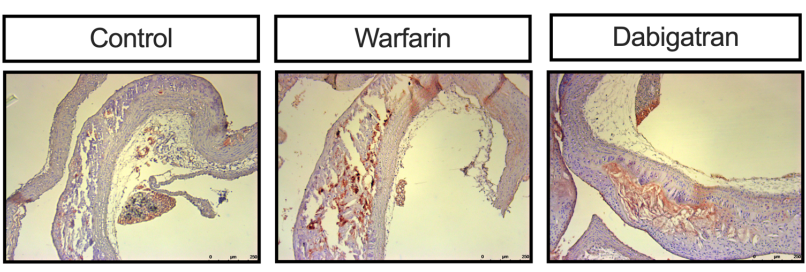

Figure 2. Long-term oral anticoagulant treatment has a differential effect on vascular calcification. A) Vascular calcification detected by $\mu \mathrm{CT}$ on whole aortic arches showed significant increased calcification after warfarin treatment compared to control $(\mathrm{p}<0.05)$ and dabigatran $(\mathrm{p}<0.01)$. B) Calcification in atherosclerotic 
lesions were visualized by Alizarin Red S and was significantly increased after warfarin treatment compared to control $(\mathrm{p}<0.05)$ and dabigatran $(\mathrm{p}<0.01)$. C) Calcification detected by $\mu \mathrm{CT}$ and Alizarin Red S strongly correlate ( $\mathrm{r} 0.72, \mathrm{p}<0.05)$. D) ${ }^{18} \mathrm{~F}-\mathrm{NaF}$ activity in whole aortic arches was significantly increased after warfarin treatment compared to control and dabigatran $(\mathrm{p}<0.05)$. E) Visualization of ${ }^{18} \mathrm{~F}-\mathrm{NaF}$ in an aortic arch by autoradiography. $\left.\mathrm{F}\right)$ In warfarin treated mice, amount of vascular calcification correlates with elevated prothrombin time ( $\mathrm{r} 0.81, \mathrm{p}<$ 0.01 ). G) Uncarboxylated matrix Gla protein (ucMGP) presence in atherosclerotic lesion was significantly increased in warfarin treated mice compared to control $(p<0.001)$ and dabigatran $(p<0.05)$. Moreover, dabigatran treatment significantly increased ucMGP presence compared to control $(\mathrm{p}<0.05)$. H) Presence of bone morphogenetic protein 4 (BMP4) in plaques was only elevated in warfarin treated mice $(\mathrm{p}<0.01$ and $\mathrm{p}<0.05$ for control and dabigatran, respectively).

dabigatran $(3.77 \pm 6.57$ total hounsfield units; $p<0.01)$ (Figure 2A). Dabigatran treatment showed a borderline significant reduction of vascular calcification compared to control $(\mathrm{p}=$ 0.07). Second, vascular calcification at 18 weeks was assessed histologically by Alizarin Red $\mathrm{S}$ staining of serial sections. Warfarin treatment showed a significant increase in plaque calcification compared to both control and dabigatran treatment $(61 \%, 33 \%$ and $21 \%$ of plaque area for warfarin, control and DE respectively; Figure $2 \mathrm{~B}$ ). Detection of calcification by $\mu \mathrm{CT}$ and Alizarin Red S showed a strong and significant correlation $(\mathrm{r}=0.72, \mathrm{p}<0.05$; Figure $2 \mathrm{C}$ ). In order to determine whether warfarin induces active calcification, calcification was investigated using the PET tracer ${ }^{18} \mathrm{~F}-\mathrm{NaF}$ (24). After 18 weeks of treatment, ${ }^{18} \mathrm{~F}-\mathrm{NaF}$ uptake by aortas, as measured ex vivo, was significantly increased by warfarin treatment as compared to control and dabigatran treatments (Figure 2D). Autoradiography showed that ${ }^{18} \mathrm{~F}-\mathrm{NaF}$ uptake predominantly localizes to the aortic arch where the atherosclerotic burden is highest (Figure 2E).

To investigate whether calcification was associated with vitamin $\mathrm{K}$ deficiency, we determined the correlation between anticoagulation (prothrombin time) and vascular calcification. Increased vascular calcification in the warfarin treated group correlated strongly with prothrombin time after 18 weeks of treatment $(r=0.81, p<0.01$; Figure $2 \mathrm{~F})$. To further test whether calcification is related to vascular vitamin $\mathrm{K}$ deficiency, ucMGP positivity in the plaques was analyzed. Warfarin significantly increased ucMGP in atherosclerotic plaques $(0.89 \%$ of plaque area) compared to control $(0.09 \%$ of plaque area; $p$ $<0.001)$ and dabigatran $(0.38 \%$ of plaque area; $\mathrm{p}<0.05)$ (Figure $2 \mathrm{G})$. UcMGP positivity was also significantly increased in dabigatran compared to control treatment (Figure 2G). To examine the contribution of MGP in the process of calcification we correlated ucMGP presence with vascular calcification detected with Alizarin Red S. UcMGP significantly correlates with vascular calcification in atherosclerotic plaques ( $\mathrm{r} 0.58, \mathrm{p}<0.01$; Supplemental Figure 1C). Moreover, effects of increased ucMGP in atherosclerotic plaques was determined by assessing bone morphogenetic protein 4 (BMP4), a known downstream effector and promoter of osteochondrogenic differentiation, was measured (25). Similar to ucMGP, warfarin treatment significantly increased BMP4 in the atherosclerotic plaque compared to both control and dabigatran (Figure 2H). In contrast to ucMGP, no difference was observed between dabigatran and control. 


\section{Chapter 5}

A

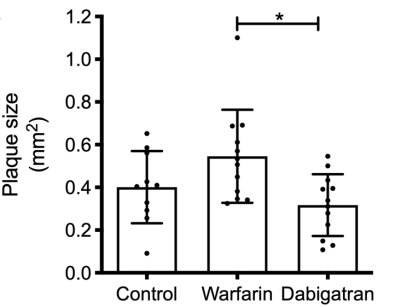

B

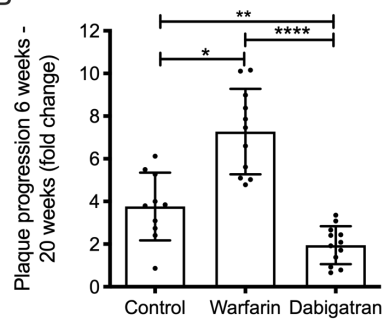

C

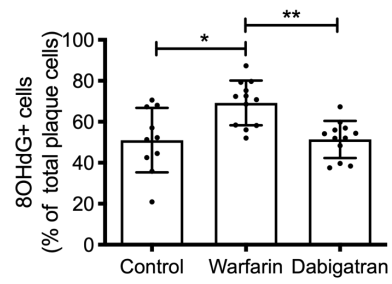

D

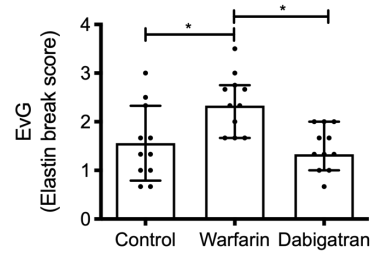

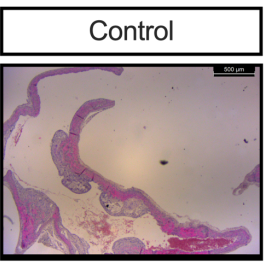
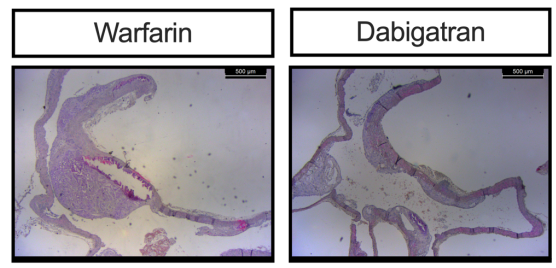
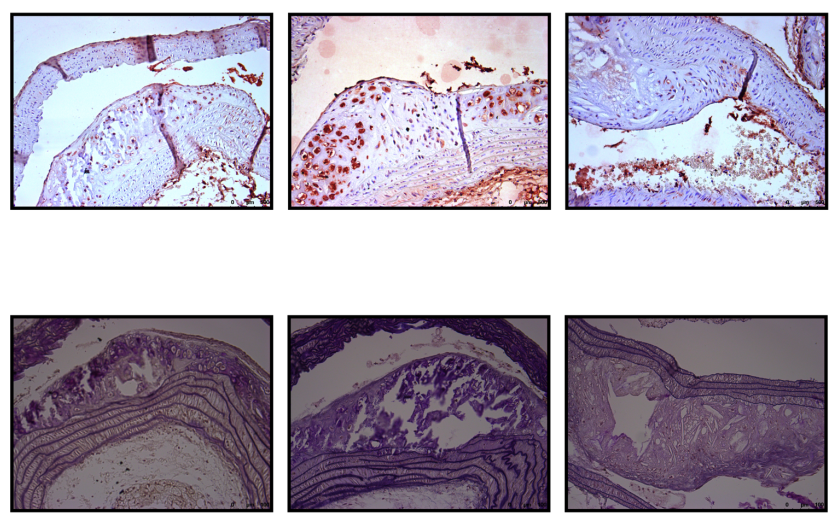

Figure 3. Long-term warfarin and not dabigatran promote plaque vulnerability. A) Aortic plaque size was significant increased after warfarin treatment compared to dabigatran treatment $(\mathrm{p}<0.05)$. B) Calculating plaque progression based on the fold change of plaque size measured at 6 and 18 weeks demonstrated significant higher plaque progression after warfarin treatment compared to control $(\mathrm{p}<0.05)$ and dabigatran $(\mathrm{p}<0.0001)$. Moreover, dabigatran treatment significantly reduced plaque progression compared to control $(\mathrm{p}<0.01)$. C) Oxidative stress in atherosclerotic lesions was significantly increased after warfarin treatment compared to control $(p<0.05)$ and dabigatran $(\mathrm{p}<0.01)$. D) Warfarin treatment resulted in significant more elastin breaks under atherosclerotic lesions compared to control and dabigatran $(\mathrm{p}<0.05)$.

\section{Differential effects of oral anticoagulation on atherogenesis.}

Warfarin treatment significantly increased atherosclerotic plaque size compared to dabigatran treatment by 2.6 -fold after 18 weeks $\left(0.54 \mathrm{~mm}^{2}\right.$ and $0.21 \mathrm{~mm}^{2}$ lesion size respectively; Figure 3A). Vascular calcification is also linked to plaque progression and used in the clinic to determine cardiovascular risk (26). Therefore, we calculated the increase in 
plaque progression as $\mathrm{x}$-fold increase in plaque size from 6 to 18 weeks of treatment. Warfarin treatment showed a significant 7.2-fold increase in plaque progression compared to a 3.7-fold increase in control $(\mathrm{p}<0.05)$ and a 1.9 -fold increase in dabigatran treatment $(\mathrm{p}$ $<0.001)$. Moreover, dabigatran treatment significantly reduced plaque progression compared to control $(\mathrm{p}<0.01)$ (Figure 3B).

Long-term VKA treatment induces a vulnerable atherosclerotic plaque phenotype.

Next, plaque composition was determined to gain insight into plaque vulnerability. Oxidative stress is considered a major cause of vascular calcification $(27,28)$. Oxidativestress, measured by $8 \mathrm{OHdG}+$ cells, was significantly increased after warfarin treatment compared to control and dabigatran (Figure 3C). No difference was observed in smooth muscle cell content ( $\alpha \mathrm{SMA}$ ), macrophages (MAC3), matrix metalloproteinases 2 (MMP2) and 9 (MMP9) or necrotic core size between any of the groups (data not shown). Elastin breaks in the tunica media underneath the atherosclerotic plaque were significantly more abundant after warfarin treatment (score 2.3) compared to control (score 1.5) or dabigatran treatment (score 1.3) $(\mathrm{p}<0.05$; Figure 3D).

Systemic inflammation is not affected by warfarin or dabigatran treatment.

After 18 weeks of treatment, cytokine levels were determined in plasma to investigate whether systemic inflammation is involved in oral anticoagulation effects on atherosclerosis. No difference in cytokines levels were found between any of the treatment regimens (supplemental Table 1).

Supplemental Table 1. Cytokine concentration in blood plasma after 18 weeks of treatment.

\begin{tabular}{|c|c|c|c|}
\hline \multicolumn{2}{|c}{ Control } & Warfarin & Dabigatran \\
\hline IFNy pg/mL & $0.339 \pm 0.161$ & $0.265 \pm 0.0569$ & $0.516 \pm 0.263$ \\
\hline IL10 pg/mL & $17.7 \pm 4.30$ & $13.2 \pm 5.70$ & $22.5 \pm 14.7$ \\
\hline IL4 pg/mL & $0.216 \pm 0.00894$ & $0.372 \pm 0.265$ & $0.245 \pm 0.0568$ \\
\hline IL5 pg/mL & $7.77 \pm 4.85$ & $7.43 \pm 4.38$ & $5.95 \pm 1.14$ \\
\hline IL6 pg/mL & $7.40 \pm 1.41$ & $10.0 \pm 4.34$ & $11.9 \pm 9.59$ \\
\hline $\begin{array}{c}\text { KC/GRO } \\
\mathbf{~ p g / m L}\end{array}$ & $36.1 \pm 9.76$ & $38.0 \pm 12.0$ & $31.3 \pm 7.35$ \\
\hline TNF $\alpha \mathbf{~ p g / m L}$ & $7.30 \pm 2.06$ & $8.25 \pm 2.20$ & $9.16 \pm 4.25$ \\
\hline
\end{tabular}

\section{Warfarin promotes an inflammatory response in vascular smooth muscle cells.}

Since warfarin treatment induced a proinflammatory phenotype at 6 weeks of treatment, we stimulated VSMC with warfarin. Proliferation and migration of VSMC is a key feature in the genesis of atherosclerosis. Unexpectedly, warfarin treatment reduced proliferation to $68 \%$ compared to control as measured by xCELLigence (Figure 4A). Reduced VSMC proliferation by warfarin was confirmed by lower EdU incorporation in a dose-dependent manner ( $\mathrm{p}<0.001$; Figure 4B). Moreover, warfarin significantly reduced VSMC migration compared to control (Figure 4C). Since warfarin treatment for 6 weeks 
A

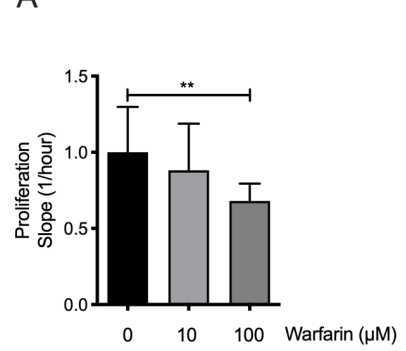

B

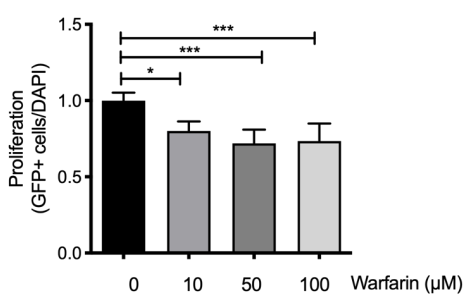

$\mathrm{F}$

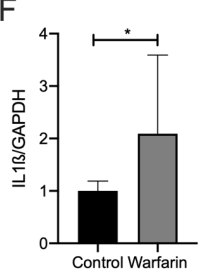

C

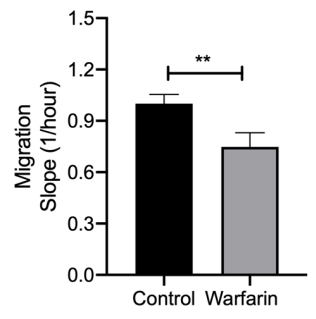

G

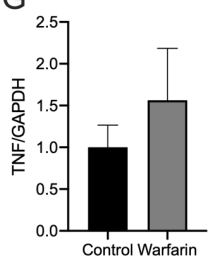

Figure 4. Differential effect of warfarin in vascular smooth muscle cells. A, B) Warfarin treatment significantly reduced VSMC proliferation and migration $(\mathrm{C})$. Warfarin $(50 \mu \mathrm{M})$ significantly promoted inflammatory mRNA levels of MCP1 (D), IFN $\gamma(\mathrm{E}), \operatorname{IL} 1 \beta$ (F) and elevated TNF $\alpha$ (G).

increased MAC3+ cells in the atherosclerotic plaque, we measured effects of warfarin on VSMC inflammation. VSMC treated with warfarin for 4 hours significantly increased MCP1 (1.6-fold; Figure 4D), IFN $\gamma$ (4.3-fold; Figure 4E) and IL1ß (2-fold; Figure 4F) mRNA levels. Although not significant, warfarin treatment also increased TNF $\alpha$ mRNA levels (Figure 4G).

Interaction of warfarin but not dabigatran with oxidized $L D L$.

The experimental animal model used to investigate the effect of oral anticoagulation was the B16/C57-Apoe - $^{-/}$which is known for its increased cholesterol levels. We tested potential synergistic effects of warfarin or dabigatran in combination with oxidized LDL (oxLDL). While oxLDL alone was a significant inducer of VSMC calcification (4.5 - 6.4fold compared to control), no effect of additional dabigatran on VSMC calcification was observed (Figure 5A). Moreover, dabigatran alone did not influence VSMC calcification (Figure 5A). On the other hand, warfarin treatment alone significantly increased VSMC calcification (2-fold; Figure 5B). Co-stimulation of warfarin with oxLDL significantly increased VSMC calcification compared to either warfarin or oxLDL alone (2.2-fold compared to oxLDL alone; Figure 5B).

Thrombin increased oxidative stress and extracellular vesicles.

Because warfarin increased oxidative stress in $A_{p o e^{-/-}}$mice, we tested whether warfarin and dabigatran also induce oxidative stress in VSMC in vitro. Intracellular oxidative stress in VSMC was determined over 30 minutes, directly after stimulation with either warfarin, thrombin or thrombin combined with dabigatran. Warfarin significantly increased oxidative 
A

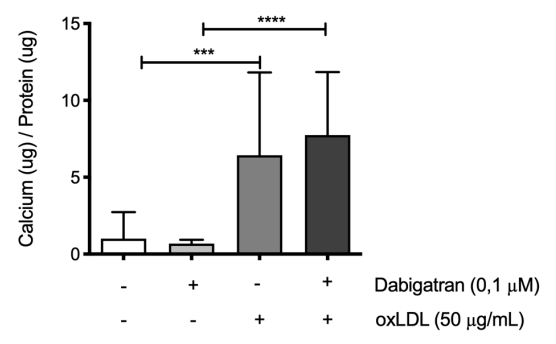

C

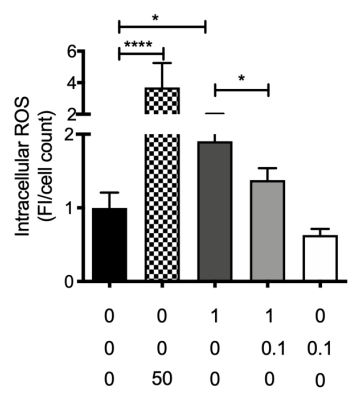

B
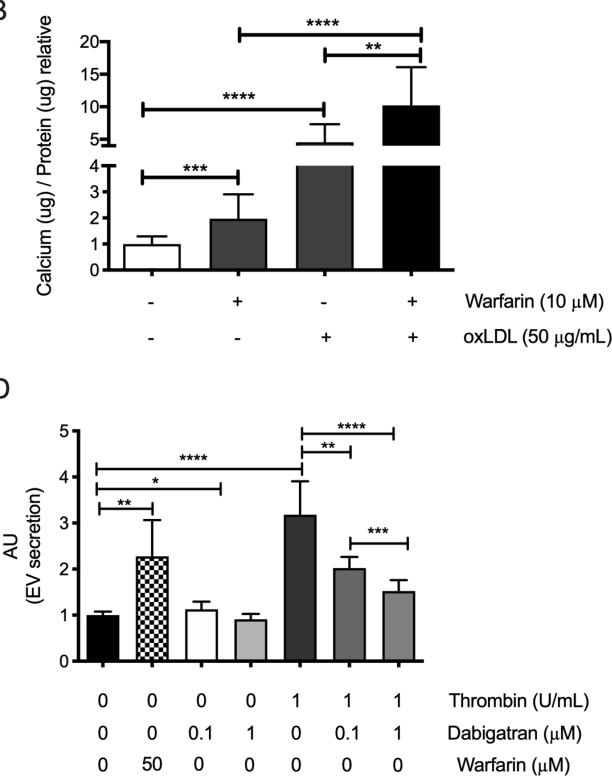

Figure 5. Interaction of warfarin and thrombin in vascular smooth muscle cells. A) Oxidized LDL (oxLDL) significantly increased calcification on VSMC. Moreover, dabigatran did not influence oxLDL mediated calcification. B) Both warfarin and oxLDL significantly increased calcification compared to control. Co-stimulation of warfarin with oxLDL resulted in significant more calcification compared to warfarin or oxLDL alone. C) Warfarin and thrombin significantly increased intracellular ROS compared to control. Thrombin induced intracellular ROS was significantly attenuated when combined with dabigatran, while dabigatran alone had no significant effect. D) Extracellular vesicles were significantly increased after both warfarin and thrombin stimulation. In contrast, high dosage of dabigatran showed a significant reduction in extracellular vesicles. Elevated extracellular vesicles after thrombin stimulation was significantly and dose dependently reduced with dabigatran.

stress compared to control (50 $\mu \mathrm{M}$ warfarin, 3.7-fold increase; Figure 5C). Thrombin significantly increased oxidative stress in VSMC compared to control treatment (1.9-fold; Figure 5C). Co-stimulation of dabigatran with thrombin significantly reduced thrombin induced oxidative stress $(\mathrm{p}<0.05$; Figure $5 \mathrm{C})$. Dabigatran stimulation alone had no effect on oxidative stress. Oxidative stress is an inducer of extracellular vesicle secretion which are known to induce VSMC calcification (22). Therefore, we analyzed the number of secreted extracellular vesicles from VSMC. Warfarin stimulation significantly increased extracellular vesicles compared to control (2.2-fold, $\mathrm{p}<0.01$; Figure 5D). Thrombin also increased extracellular vesicle release significantly (3.1-fold), which was dose-dependently reduced after co-stimulation with dabigatran (2-fold for $0.1 \mu \mathrm{M}$ dabigatran and 1.5-fold for $1 \mu \mathrm{M}$ dabigatran compared to control; Figure 5D). Moreover, dabigatran stimulation alone significantly reduced extracellular vesicles compared to control ( 0.9 -fold of control for $1 \mu \mathrm{M}$ dabigatran; Figure 5D). 
Differently expressed genes after thrombin and warfarin stimulation in vascular smooth muscle cells.

To further analyze the effects of warfarin and thrombin on VSMC, we performed transcriptomic analysis after 4 hours of stimulation of warfarin and thrombin compared to control. While warfarin affected 44 transcripts $(27$ genes increased expression and 17 reduced compared to control), thrombin altered a total of 1582 transcripts (expression of 979 genes were increased and expression of 603 genes were reduced compared to control). Altered genes were compared with known datasets associated with atherosclerosis development (1133 genes; Table 2). Thrombin significantly changed expression of 107 genes in this transcriptome while warfarin only significantly altered expression of 4 genes. FABP5 and IL1ß were significantly altered for both warfarin and thrombin. Transcriptomic analysis was also compared with a known inflammatory gene dataset (428 genes; Table 3) because of the important role of inflammation in atherosclerosis together with the observed inflammatory effect of warfarin in vivo and in vitro. Here, thrombin altered 43 genes of which only 3 were also altered by warfarin stimulation (IL1ß, leukemia inhibitory factor (LIF), CXCL2).

Table 2. Altered gene transcripts of an atherosclerotic dataset of genes.

\begin{tabular}{|c|c|c|c|c|c|c|c|c|}
\hline \multicolumn{9}{|c|}{ Atherosclerosis } \\
\hline \multirow{2}{*}{ Gene } & Thrombin & Warfarin & \multirow{2}{*}{ Gene } & Thrombin & Warfarin & \multirow{2}{*}{ Gene } & Thrombin & Warfarin \\
\hline & LogFC & $\log F C$ & & $\operatorname{LogFC}$ & $\log F C$ & & $\operatorname{LogFC}$ & $\log F C$ \\
\hline $\begin{array}{c}\text { ABCA } \\
1\end{array}$ & -1.29 & 0.02 & $\begin{array}{c}\text { GREM } \\
1\end{array}$ & 2.15 & 0.20 & $\begin{array}{c}\text { PLA2G } \\
4 A \\
\end{array}$ & 0.67 & 0.11 \\
\hline ABR & 0.64 & 0.18 & HAS2 & 2.00 & 0.44 & PLAT & 0.67 & 0.14 \\
\hline $\begin{array}{c}\text { ADAM } \\
8\end{array}$ & -0.75 & -0.08 & $\begin{array}{c}\text { HILPD } \\
\text { A }\end{array}$ & 0.88 & -0.06 & PLAUR & 1.52 & 0.05 \\
\hline $\begin{array}{c}\text { ADAM } \\
\text { TS1 }\end{array}$ & 0.66 & -0.18 & $\begin{array}{c}\text { HMGA } \\
1\end{array}$ & 0.62 & 0.09 & PNP & 0.96 & 0.04 \\
\hline $\begin{array}{c}\text { ADAM } \\
\text { TS5 }\end{array}$ & -2.08 & -0.08 & $\begin{array}{c}\text { HS3ST } \\
1\end{array}$ & 1.28 & 0.12 & PPARG & 1.28 & 0.09 \\
\hline AHRR & 0.01 (NS) & $0.78 *$ & $\begin{array}{c}\text { HSD11 } \\
\text { B1 }\end{array}$ & 0.62 & 0.12 & PTGS1 & 0.62 & 0.19 \\
\hline $\begin{array}{c}\text { APOL } \\
6 \\
\end{array}$ & -0.83 & -0.03 & HSPA2 & 1.95 & -0.16 & PTK2B & -0.88 & -0.12 \\
\hline BDNF & -0.60 & -0.34 & ICAM1 & 0.83 & 0.24 & RGS2 & 0.73 & -0.14 \\
\hline BMP4 & -0.74 & -0.16 & IL11 & 0.60 & -0.04 & $\begin{array}{c}\text { SAMD4 } \\
\text { A } \\
\end{array}$ & 1.45 & 0.01 \\
\hline CASP1 & -0.71 & 0.18 & IL1B & 1.30 & $0.76 *$ & SAMD9 & 0.79 & 0.05 \\
\hline CASP3 & 0.73 & -0.06 & IL33 & 0.83 & 0.05 & $\begin{array}{c}\text { SERPI } \\
\text { NE1 }\end{array}$ & 1.69 & -0.12 \\
\hline CAV1 & 0.85 & 0.06 & IL6 & 0.76 & -0.25 & $\begin{array}{c}\text { SLC16 } \\
\text { A3 }\end{array}$ & 0.94 & -0.16 \\
\hline
\end{tabular}




\begin{tabular}{|c|c|c|c|c|c|c|c|c|}
\hline CCL2 & 1.47 & 0.24 & IRS2 & -0.81 & -0.04 & $\begin{array}{c}\text { SLC17 } \\
\text { A5 }\end{array}$ & 0.79 & -0.14 \\
\hline CD44 & 0.67 & 0.17 & ISG20 & 1.46 & 0.09 & $\begin{array}{c}\text { SLC6A } \\
4\end{array}$ & -0.67 & 0.09 \\
\hline $\begin{array}{c}\text { CDKN } \\
1 C\end{array}$ & -0.82 & 0.15 & ITIH4 & -0.70 & 0.13 & SMAD3 & 0.78 & 0.00 \\
\hline CHDH & 1.53 & -0.12 & KLF4 & 0.88 & -0.22 & SOCS1 & 0.65 & 0.37 \\
\hline $\begin{array}{c}\text { CREB } \\
3\end{array}$ & 0.60 & -0.09 & KLF5 & -0.72 & -0.32 & SOCS3 & 0.78 & 0.10 \\
\hline CRY1 & 0.99 & -0.32 & LDB2 & -1.19 & 0.06 & SOD2 & 0.99 & 0.04 \\
\hline CTF1 & -0.83 & 0.08 & LIPE & -0.70 & 0.06 & $\begin{array}{c}\text { ST8SIA } \\
1\end{array}$ & -1.56 & 0.08 \\
\hline $\begin{array}{c}\text { CXCL } \\
1\end{array}$ & 2.49 & 0.41 & LMNA & 0.95 & -0.08 & $\begin{array}{c}\text { TCF7L } \\
2\end{array}$ & -0.91 & -0.38 \\
\hline $\begin{array}{c}\text { DDAH } \\
1\end{array}$ & 1.45 & -0.25 & LRP8 & 0.67 & 0.26 & TFPI2 & 0.97 & 0.08 \\
\hline DUSP1 & 0.76 & -0.29 & $\begin{array}{c}\text { MAP2 } \\
\text { K1 }\end{array}$ & 0.89 & -0.05 & THBD & 2.71 & 0.19 \\
\hline ECE2 & 0.71 & -0.09 & $\begin{array}{c}\text { MAP3 } \\
\text { K5 }\end{array}$ & -1.17 & 0.16 & $\begin{array}{c}\text { TNFRS } \\
\text { F11B }\end{array}$ & 0.76 & -0.23 \\
\hline EDN1 & -1.25 & -0.20 & MPRIP & 0.89 & -0.19 & $\begin{array}{c}\text { TNFRS } \\
\text { F12A }\end{array}$ & 1.03 & -0.39 \\
\hline $\begin{array}{c}\text { EDNR } \\
\text { A }\end{array}$ & -0.99 & 0.32 & MTSS1 & 1.81 & 0.23 & $\begin{array}{c}\text { TNFRS } \\
\text { F14 }\end{array}$ & -0.72 & 0.14 \\
\hline EFNB1 & -0.77 & 0.17 & МYH9 & 0.63 & -0.28 & $\begin{array}{c}\text { TNFRS } \\
\text { F4 }\end{array}$ & 0.29 (NS) & $1.22 *$ \\
\hline EGFR & 0.78 & 0.08 & $\begin{array}{c}\text { NCEH } \\
1\end{array}$ & 0.71 & 0.21 & $\begin{array}{c}\text { TNFSF } \\
4\end{array}$ & 1.10 & -0.05 \\
\hline ELK1 & 0.61 & 0.14 & $\begin{array}{c}\text { NOTC } \\
\text { H1 }\end{array}$ & 0.78 & 0.21 & $\begin{array}{c}\text { TNFSF } \\
9\end{array}$ & 0.66 & 0.01 \\
\hline ENPP1 & 0.63 & -0.12 & NOV & 0.69 & -0.09 & $\begin{array}{c}\text { TRAF3 } \\
\text { IP2 }\end{array}$ & 0.88 & 0.24 \\
\hline ETS1 & 0.68 & 0.02 & NR1D1 & -0.82 & 0.10 & TRIB3 & -1.57 & -0.05 \\
\hline FABP5 & -0.70 & $0.60 *$ & NR4A3 & 3.41 & 0.18 & UNC5B & -0.76 & -0.05 \\
\hline FGF2 & -0.96 & -0.25 & NRG1 & -1.47 & -0.16 & VCL & 1.05 & -0.15 \\
\hline FGFR1 & 0.60 & 0.00 & NUP62 & 0.69 & -0.13 & $\begin{array}{c}\text { VKOR } \\
\text { C1 }\end{array}$ & 0.61 & 0.01 \\
\hline FOS & -1.00 & -0.23 & $\begin{array}{c}\text { PDGF } \\
\text { A }\end{array}$ & 1.50 & -0.09 & VWF & 1.72 & 0.21 \\
\hline FST & 0.95 & -0.28 & $\begin{array}{c}\text { PECA } \\
\text { M1 }\end{array}$ & 1.12 & 0.15 & $\begin{array}{c}\text { WNT5 } \\
\text { A }\end{array}$ & -0.69 & 0.08 \\
\hline FURIN & 0.87 & 0.20 & $\begin{array}{c}\text { PIK3C } \\
\text { D }\end{array}$ & 1.15 & 0.00 & XPR1 & 0.94 & -0.20 \\
\hline GDF15 & -0.71 & -0.05 & & & & & & \\
\hline
\end{tabular}

Increased (red) and reduction (blue) compared to control. All thrombin, except for (NS), altered gene expressions were significant different compared to control and * present significant difference of warfarin compared to control. 
Table 3. Altered gene transcripts of an inflammatory dataset.

\begin{tabular}{|c|c|c|c|c|c|}
\hline \multicolumn{6}{|c|}{ Inflammation } \\
\hline & Thrombin & Warfarin & & Thrombin & Warfarin \\
\hline Gene & $\operatorname{LogFC}$ & $\operatorname{logFC}$ & Gene & $\operatorname{LogFC}$ & $\operatorname{logFC}$ \\
\hline AQP3 & 0.90 & 0.08 & NRG1 & -1.47 & -0.16 \\
\hline BDKRB1 & 0.64 & 0.20 & PDGFA & 1.50 & -0.09 \\
\hline BDNF & -0.60 & -0.34 & PECAM1 & 1.12 & 0.15 \\
\hline BMP4 & -0.74 & -0.16 & PLAT & 0.67 & 0.14 \\
\hline CASP1 & -0.71 & 0.18 & PLAUR & 1.52 & 0.05 \\
\hline CCL2 & 1.47 & 0.24 & PPARG & 1.28 & 0.09 \\
\hline CD44 & 0.67 & 0.17 & SERPINE1 & 1.69 & -0.12 \\
\hline CD83 & 0.63 & 0.40 & SETD7 & 0.66 & 0.01 \\
\hline CXCL1 & 2.49 & 0.41 & SMAD3 & 0.78 & 0.00 \\
\hline CXCL2 & 1.68 & $1.04 *$ & SOCS1 & 0.65 & 0.37 \\
\hline CXCL6 & 1.55 & 0.47 & SOCS3 & 0.78 & 0.10 \\
\hline EDN1 & -1.25 & -0.20 & TFPI2 & 0.97 & 0.08 \\
\hline EFNB1 & -0.77 & 0.17 & THBD & 2.71 & 0.19 \\
\hline FGF2 & -0.96 & -0.25 & TIMP4 & 0.65 & 0.12 \\
\hline ICAM1 & 0.83 & 0.24 & TNFAIP3 & 1.70 & -0.03 \\
\hline IL1B & 1.30 & $0.76 *$ & TNFRSF11B & 0.76 & -0.23 \\
\hline IL1RAP & -1.19 & 0.13 & TNFRSF12A & 1.03 & -0.39 \\
\hline IL33 & 0.83 & 0.05 & $\mathrm{UCN}$ & -1.04 & -0.01 \\
\hline IL4R & 0.74 & 0.12 & VWF & 1.72 & 0.21 \\
\hline IL6 & 0.76 & -0.25 & WDR1 & 0.90 & -0.08 \\
\hline LIAS & -0.61 & -0.02 & YWHAH & 0.73 & -0.06 \\
\hline LIF & 1.65 & $0.75^{*}$ & & & \\
\hline
\end{tabular}

Increased (red) and reduction (blue) compared to control. All thrombin altered gene expressions were significant different compared to control and * present significant difference of warfarin compared to control.

\section{Discussion}

In the presented study, we demonstrate that warfarin and dabigatran have differential effects on atherosclerosis. Warfarin treatment accelerated plaque inflammation, oxidative stress, calcification activity and plaque progression. By contrast dabigatran had no effect on vascular inflammation or calcification, and reduced plaque progression in vivo. These data suggests that the choice of anticoagulant in patients at risk of venous or arterial thrombosis 
Warfarin promotes atherosclerotic disease activity by increased presence of calcification and calcification activity. Recently, greater calcification volume but not higher density was associated with increased cardiovascular disease risk (29). Our data show that warfarin increases calcification volume in atherosclerotic plaques, suggesting that warfarin increases plaque vulnerability. Dabigatran had no effect on calcification and even reduced plaque progression, implying that local effects play key roles in cardiovascular disease. It is known that warfarin as with all VKA inhibits carboxylation of vitamin K dependent proteins, thereby reducing coagulation tendency, at the expense of MGP activity (30). MGP is one of the strongest vascular calcification inhibitors (31) and reduced activation of MGP by warfarin appears to tip the balance towards calcification. Carboxylated MGP binds to BMP4, thereby reducing osteochondrogenic differentiation (25). Also, in our animal model, warfarin treatment increased the presence of BMP4 in the atherosclerotic plaque. Moreover, dabigatran increased ucMGP presence in atherosclerotic plaque, but, in contrast to warfarin, did not affect BMP4 presence in atherosclerotic plaque. These data suggest that elevated levels of ucMGP point towards a coping mechanism for elevated calcification pressure (4, 32), while carboxylated MGP is present in dabigatran treated animals.

MGP binds to calcium crystals and extracellular vesicles, blocking further growth of calcification (33). Warfarin treatment increased ucMGP already in early atherosclerotic lesions, even before calcification could be visualized. Active calcification, detected by ${ }^{18} \mathrm{~F}$ $\mathrm{NaF}$, is associated with increased plaque vulnerability (34). We show that mice treated with warfarin displayed higher ${ }^{18} \mathrm{~F}-\mathrm{NaF}$ positivity in the vasculature compared to dabigatran and control, indicating that VKA induces active mineralization. Our data is in line with recently published clinical studies comparing VKA, NOAC or no oral anticoagulant treatment on vascular calcification. VKA treatment showed higher prevalence of vascular and valvular calcification, which was not seen in NOAC treated patients (7). Moreover, plaque burden and prevalence of high-risk plaques have been shown to be increased in patients using VKA compared to NOAC treatment (15). Although caution should be taken with generalizing NOAC treatments.

Oxidative stress has been shown to increase vascular calcification development (28). Warfarin increases oxidative stress in vivo and in vitro, whereas dabigatran reduced thrombin induced oxidative stress. VKA has been put forward to induce oxidative stress via inhibition of the vitamin K cycle (35). VKA are known to interact with cytochrome P450 (CYP) and our transcriptomic data show elevated CYP1B1 transcript after warfarin treatment. CYP1B1 has been shown increase oxidative stress in VSMC (36). Dabigatran on the other hand, has been shown to block oxidative stress in mice thereby improving nitric oxide (NO) synthesis $(37,45)$. Extracellular vesicles from VSMC play an important role in the initiation of calcification (22) and both warfarin and thrombin increased extracellular vesicle secretion. Taken together, warfarin induces impaired carboxylation of MGP, and increases oxidative stress and extracellular vesicle release, thereby supporting vascular calcification. In contrast, dabigatran inhibits the detrimental side effects of thrombin on VSMC. 
Both thrombin and warfarin increased IL1 1 transcripts, which has been associated with exacerbation of atherosclerosis (38). Microcalcification can promote inflammation (39) and inflammation can promote calcification (40). We show increased presence of macrophages in early atherosclerotic lesions and elevated inflammatory markers in VSMC after warfarin treatment. Moreover, we demonstrate that warfarin treatment increases ${ }^{18} \mathrm{~F}-\mathrm{NaF}$ uptake, which is indicative of presence of microcalcification. Warfarin has previously been shown to have immunomodulatory effects, including promoting an inflammatory environment (41, 42). It has been demonstrated that calcified VSMC initiate macrophage migration and promote a pro-inflammatory environment (39). In early atherosclerotic lesions, hydroxyapatite nanocrystals are present, associated with initial mineralization $(40,43)$. Additionally, calcium-crystals activate macrophages thereby promoting a pro-inflammatory environment (44) and gaining osteogenic activity (40). Consequently, inflammatory macrophages promote calcification via release of $\mathrm{TNF} \alpha$. In line with these data, we confirm that mice treated with warfarin had more macrophages in early atherosclerotic lesions. In conclusion, macrophage rich plaques display increased osteogenic activity, further enhancing the strong correlation between inflammation and vascular calcification.

Thrombin can affect atherosclerosis development by promoting inflammation via cytokine production, proliferation and cytoskeletal rearrangement of VSMC, fibroblast stimulation and NO synthesis (45-47). In atherosclerosis, thrombin also promotes leucocyte recruitment, while reduction of thrombin by approximately $50 \%$ reduced macrophages content (18). Here, we showed that dabigatran reduced atherogenesis and that thrombin inhibition induced oxidative stress and extracellular vesicle secretion in vitro. Moreover, thrombin stimulation in VSMC increased a wide range of transcript genes associated with atherosclerosis and inflammation. Thrombin binds and cleaves the N-terminal exodomain of PAR1 thereby initiating PAR1 signaling. Dabigatran inhibits thrombin, thereby attenuating thrombin-induced PAR signaling resulting in diminished or altered atherosclerosis. Indeed, dabigatran has been shown to reduce thrombin induced inflammatory response $(48,49)$.

This study has several limitations. The atherosclerotic $A p o e^{-/-}$mice model is a widely accepted animal model for studying atherosclerosis. While there is great similarities in morphology of plaques compared to humans, atherosclerotic plaques in mice will not rupture. Therefore, potential consequences of myocardial infarction or stroke are absent in this model. Dosage of dabigatran is higher in mice compared to human's due to lower affinity of dabigatran in rodents (19). Moreover, our dosage of warfarin in mice (eg, $3 \mathrm{mg} / \mathrm{g}$ and mice eat approximately 4 gram per day, daily intake of 10-15 mg per day) exceeded dose in humans (5 mg per day/70 kg) (50). Nevertheless, warfarin was combined with elevated vitamin K1 to prevent bleeding and was used to study VKA effects on the vessel wall. In the present study, we determined the differential effects of warfarin and dabigatran on the vessel wall and possible consequence in atherosclerosis development.

In conclusion, VKA aggravates atherogenesis via its direct effects on VSMC. Our data show that VKA increases atherosclerotic disease activity, increasing plaque inflammation, 
oxidative stress and calcification activity; effects that translate into faster plaque progression. In contrast, these effects are not observed with dabigatran, which appeared to have a beneficial effect on disease progression. Our findings suggest that the choice of anticoagulant in patients at risk of arterial of venous thrombosis may have important off target effects on bystander atherosclerotic disease, with further studies in humans now required.

\section{Disclosure}

JR is supported by Boehringer-Ingelheim.

\section{Acknowledgements}

Dabigatran etexilate was kindly provided by Boehringer-Ingelheim. This work was financed by Dutch Thrombosis Society (2014.02) and by the Norwegian Research Council and Nattopharma ASA.

\section{References}

1. Martin-Ventura JL, Rodrigues-Diez R, Martinez-Lopez D, Salaices M, Blanco-Colio LM, Briones AM. Oxidative Stress in Human Atherothrombosis: Sources, Markers and Therapeutic Targets. Int J Mol Sci. 2017 Nov 3;18(11).

2. Bentzon JF, Otsuka F, Virmani R, Falk E. Mechanisms of plaque formation and rupture. Circ Res. 2014 Jun 6;114(12):1852-66.

3. Kapustin AN, Schoppet M, Schurgers LJ, Reynolds JL, McNair R, Heiss A, et al. Prothrombin Loading of Vascular Smooth Muscle Cell-Derived Exosomes Regulates Coagulation and CalcificationHighlights. Arterioscler Thromb Vasc Biol. American Heart Association, Inc; 2017 Mar 1;37(3):e22-e32.

4. Schurgers LJ, Teunissen KJF, Knapen MHJ, Kwaijtaal M, van Diest R, Appels A, et al. Novel conformation-specific antibodies against matrix gamma-carboxyglutamic acid (Gla) protein: undercarboxylated matrix Gla protein as marker for vascular calcification. Arterioscler Thromb Vasc Biol. 2005 Aug;25(8):1629-33.

5. Murshed M, Schinke T, McKee MD, Karsenty G. Extracellular matrix mineralization is regulated locally; different roles of two gla-containing proteins. J Cell Biol. 2004 Jun 7;165(5):625-30.

6. Schurgers LJ, Cranenburg ECM, Vermeer C. Matrix Gla-protein: the calcification inhibitor in need of vitamin K. Thromb Haemost. 2008 Oct;100(4):593-603.

7. Peeters F, Dudink E, Kimenai D, Weijs B, Altintas S, Heckman L, et al. Vitamin K Antagonists, NonVitamin K Antagonist Oral Anticoagulants, and Vascular Calcification in Patients with Atrial Fibrillation. TH Open. Georg Thieme Verlag KG; 2018 Oct 1;02(04):e391-8.

8. Schurgers LJ, Joosen IA, Laufer EM, Chatrou MLL, Herfs M, Winkens MHM, et al. Vitamin Kantagonists accelerate atherosclerotic calcification and induce a vulnerable plaque phenotype. PLoS ONE. 2012;7(8):e43229.

9. Shioi A, Ikari Y. Plaque Calcification During Atherosclerosis Progression and Regression. JAT. Japan Atherosclerosis Society; 2018 Apr 1;25(4):294-303.

10. Lin TC, Tintut Y, Lyman A, Mack W, Demer LL, Hsiai TK. Mechanical response of a calcified plaque model to fluid shear force. Ann Biomed Eng. Kluwer Academic Publishers-Plenum Publishers; 2006 Oct;34(10):1535-41.

11. Ehara S, Kobayashi Y, Yoshiyama M, Shimada K, Shimada Y, Fukuda D, et al. Spotty calcification typifies the culprit plaque in patients with acute myocardial infarction: an intravascular ultrasound study. Circulation. 2004 Nov 30;110(22):3424-9.

12. Vengrenyuk Y, Carlier S, Xanthos S, Cardoso L, Ganatos P, Virmani R, et al. A hypothesis for vulnerable plaque rupture due to stress-induced debonding around cellular microcalcifications in thin fibrous caps. Proc Natl Acad Sci USA. 2006 Oct 3;103(40):14678-83.

13. Criqui MH, Denenberg JO, Ix JH, McClelland RL, Wassel CL, Rifkin DE, et al. Calcium density of coronary artery plaque and risk of incident cardiovascular events. JAMA. American Medical Association; 2014 Jan 15;311(3):271-8. 
14. Dweck MR, Jenkins WSA, Vesey AT, Pringle MAH, Chin CWL, Malley TS, et al. 18F-sodium fluoride uptake is a marker of active calcification and disease progression in patients with aortic stenosis. Circ Cardiovasc Imaging. 2014 Mar;7(2):371-8.

15. Plank F, Beyer C, Friedrich G, Stühlinger M, Hintringer F, Dichtl W, et al. Influence of vitamin K antagonists and direct oral anticoagulation on coronary artery disease: A CTA analysis. International Journal of Cardiology. Elsevier B.V; 2018 Jun 1;260(C):11-5.

16. Alberelli MA, De Candia E. Functional role of protease activated receptors in vascular biology. Vascul Pharmacol. 2014 Aug;62(2):72-81.

17. Borissoff JI, Spronk HMH, Heeneman S, Cate ten H. Is thrombin a key player in the "coagulationatherogenesis" maze? Cardiovasc Res. 2009 Jun 1;82(3):392-403.

18. Borissoff JI, Otten JJT, Heeneman S, Leenders P, van Oerle R, Soehnlein O, et al. Genetic and pharmacological modifications of thrombin formation in apolipoprotein e-deficient mice determine atherosclerosis severity and atherothrombosis onset in a neutrophil-dependent manner. PLoS ONE. 2013;8(2):e55784.

19. Kadoglou NPE, Moustardas P, Katsimpoulas M, Kapelouzou A, Kostomitsopoulos N, Schafer K, et al. The beneficial effects of a direct thrombin inhibitor, dabigatran etexilate, on the development and stability of atherosclerotic lesions in apolipoprotein E-deficient mice : dabigatran etexilate and atherosclerosis. Cardiovasc Drugs Ther. 2012 Oct;26(5):367-74.

20. Price PA, Kaneda Y. Vitamin K counteracts the effect of warfarin in liver but not in bone. Thromb Res. 1987 Apr 1;46(1):121-31.

21. Reynolds JL, Joannides AJ, Skepper JN, McNair R, Schurgers LJ, Proudfoot D, et al. Human vascular smooth muscle cells undergo vesicle-mediated calcification in response to changes in extracellular calcium and phosphate concentrations: a potential mechanism for accelerated vascular calcification in ESRD. J Am Soc Nephrol. 2004 Nov;15(11):2857-67.

22. Kapustin AN, Chatrou MLL, Drozdov I, Zheng Y, Davidson SM, Soong D, et al. Vascular smooth muscle cell calcification is mediated by regulated exosome secretion. Circ Res. Lippincott Williams \& Wilkins; 2015 Apr 10;116(8):1312-23.

23. Smyth GK. limma: Linear Models for Microarray Data. In: Gentleman R, Carey VJ, Huber W, Irizarry RA, Dudoit S, editors. Bioinformatics and Computational Biology Solutions Using R and Bioconductor. New York, NY: Springer New York; 2005. pp. 397-420. (Bioinformatics and Computational Biology Solutions Using R and Bioconductor).

24. Joshi NV, Vesey AT, Williams MC, Shah ASV, Calvert PA, Craighead FHM, et al. 18F-fluoride positron emission tomography for identification of ruptured and high-risk coronary atherosclerotic plaques: a prospective clinical trial. Lancet. $2014 \mathrm{Feb} 22 ; 383(9918): 705-13$.

25. Boström KI, Jumabay M, Matveyenko A, Nicholas SB, Yao Y. Activation of vascular bone morphogenetic protein signaling in diabetes mellitus. Circ Res. 2011 Feb 18;108(4):446-57.

26. Raggi P, Callister TQ, Shaw LJ. Progression of coronary artery calcium and risk of first myocardial infarction in patients receiving cholesterol-lowering therapy. Arterioscler Thromb Vasc Biol. American Heart Association, Inc; 2004 Jul;24(7):1272-7.

27. Byon CH, Javed A, Dai Q, Kappes JC, Clemens TL, Darley-Usmar VM, et al. Oxidative stress induces vascular calcification through modulation of the osteogenic transcription factor Runx 2 by AKT signaling. J Biol Chem. 2008 May 30;283(22):15319-27.

28. Byon CH, Heath JM, Chen Y. Redox signaling in cardiovascular pathophysiology: A focus on hydrogen peroxide and vascular smooth muscle cells. Redox Biol. 2016 Oct;9:244-53.

29. Criqui MH, Knox JB, Denenberg JO, Forbang NI, McClelland RL, Novotny TE, et al. Coronary Artery Calcium Volume and Density: Potential Interactions and Overall Predictive Value: The Multi-Ethnic Study of Atherosclerosis. JACC Cardiovasc Imaging. 2017 Aug;10(8):845-54.

30. van Gorp RH, Schurgers LJ. New Insights into the Pros and Cons of the Clinical Use of Vitamin K Antagonists (VKAs) Versus Direct Oral Anticoagulants (DOACs). Nutrients. 2015;7(11):9538-57.

31. Luo G, Ducy P, McKee MD, Pinero GJ, Loyer E, Behringer RR, et al. Spontaneous calcification of arteries and cartilage in mice lacking matrix GLA protein. Nature. 1997 Mar 6;386(6620):78-81.

32. Proudfoot D, Shanahan CM. Molecular mechanisms mediating vascular calcification: role of matrix Gla protein. Nephrology (Carlton). John Wiley \& Sons, Ltd (10.1111); 2006 Oct;11(5):455-61.

33. Schurgers LJ, Spronk HMH, Skepper JN, Hackeng TM, Shanahan CM, Vermeer C, et al. Posttranslational modifications regulate matrix Gla protein function: importance for inhibition of vascular smooth muscle cell calcification. J Thromb Haemost. 2007 Dec;5(12):2503-11.

34. Irkle A, Vesey AT, Lewis DY, Skepper JN, Bird JLE, Dweck MR, et al. Identifying active vascular microcalcification by (18)F-sodium fluoride positron emission tomography. Nat Comms. 2015;6:7495. 
35. Petsophonsakul P, Furmanik M, Forsythe R, Dweck M, Schurink GW, Natour E, et al. Role of Vascular Smooth Muscle Cell Phenotypic Switching and Calcification in Aortic Aneurysm Formation. Arterioscler Thromb Vasc Biol. Lippincott Williams \& Wilkins Hagerstown, MD; 2019 Jul;39(7):1351-68.

36. Yaghini FA, Song CY, Lavrentyev EN, Ghafoor HUB, Fang XR, Estes AM, et al. Angiotensin II-Induced Vascular Smooth Muscle Cell Migration and Growth Are Mediated by Cytochrome P450 1B1-Dependent Superoxide Generation. Hypertension. 2010 Jun;55(6):1461-7.

37. Pingel S, Tiyerili V, Mueller J, Werner N, Nickenig G, Mueller C. Thrombin inhibition by dabigatran attenuates atherosclerosis in ApoE deficient mice. Arch Med Sci. 2014 Feb 24;10(1):154-60.

38. Libby P. Interleukin-1 Beta as a Target for Atherosclerosis Therapy: Biological Basis of CANTOS and Beyond. J Am Coll Cardiol. 2017 Oct 31;70(18):2278-89.

39. Chatrou MLL, Cleutjens JP, van der Vusse GJ, Roijers RB, Mutsaers PHA, Schurgers LJ. Intra-Section Analysis of Human Coronary Arteries Reveals a Potential Role for Micro-Calcifications in Macrophage Recruitment in the Early Stage of Atherosclerosis. PLoS ONE. 2015;10(11):e0142335.

40. Aikawa E, Nahrendorf M, Figueiredo J-L, Swirski FK, Shtatland T, Kohler RH, et al. Osteogenesis associates with inflammation in early-stage atherosclerosis evaluated by molecular imaging in vivo. Circulation. 2007 Dec 11;116(24):2841-50.

41. Mirkov I, Popov Aleksandrov A, Demenesku J, Ninkov M, Mileusnic D, Kataranovski D, et al. Warfarin affects acute inflammatory response induced by subcutaneous polyvinyl sponge implantation in rats. Cutan Ocul Toxicol. Taylor \& Francis; 2017 Sep;36(3):283-8.

42. Belij S, Miljković D, Popov A, Subota V, Timotijević G, Slavić M, et al. Effects of subacute oral warfarin administration on peripheral blood granulocytes in rats. Food Chem Toxicol. 2012 May;50(5):1499-507.

43. Roijers RB, Debernardi N, Cleutjens JPM, Schurgers LJ, Mutsaers PHA, van der Vusse GJ. Microcalcifications in early intimal lesions of atherosclerotic human coronary arteries. The American Journal of Pathology. 2011 Jun;178(6):2879-87.

44. Nadra I, Mason JC, Philippidis P, Florey O, Smythe CDW, McCarthy GM, et al. Proinflammatory Activation of Macrophages by Basic Calcium Phosphate Crystals via Protein Kinase C and MAP Kinase Pathways. Circ Res. 2005 Jun 24;96(12):1248-56.

45. Lee I-O, Kratz MT, Schirmer SH, Baumhäkel M, Böhm M. The effects of direct thrombin inhibition with dabigatran on plaque formation and endothelial function in apolipoprotein E-deficient mice. J Pharmacol Exp Ther. 2012 Nov;343(2):253-7.

46. Yufu T, Hirano K, Bi D, Hirano M, Nishimura J, Iwamoto Y, et al. Rac1 Regulation of Surface Expression of Protease-Activated Receptor-1 and Responsiveness to Thrombin in Vascular Smooth Muscle Cells. Arterioscler Thromb Vasc Biol. 2005 Jul;25(7):1506-11.

47. Ivey ME, Little PJ. Thrombin regulates vascular smooth muscle cell proteoglycan synthesis via PAR-1 and multiple downstream signalling pathways. Thromb Res. 2008;123(2):288-97.

48. Spronk HMH, de Jong AM, Verheule S, de Boer HC, Maass AH, Lau DH, et al. Hypercoagulability causes atrial fibrosis and promotes atrial fibrillation. Eur Heart J. The Oxford University Press; 2016 Apr $12 ;:$ ehw119.

49. Kopec AK, Joshi N, Towery KL, Kassel KM, Sullivan BP, Flick MJ, et al. Thrombin inhibition with dabigatran protects against high-fat diet-induced fatty liver disease in mice. J Pharmacol Exp Ther. American Society for Pharmacology and Experimental Therapeutics; 2014 Nov;351(2):288-97.

50. Krüger T, Oelenberg S, Kaesler N, Schurgers LJ, van de Sandt AM, Boor P, et al. Warfarin induces cardiovascular damage in mice. Arterioscler Thromb Vasc Biol. 2013 Nov;33(11):2618-24. 


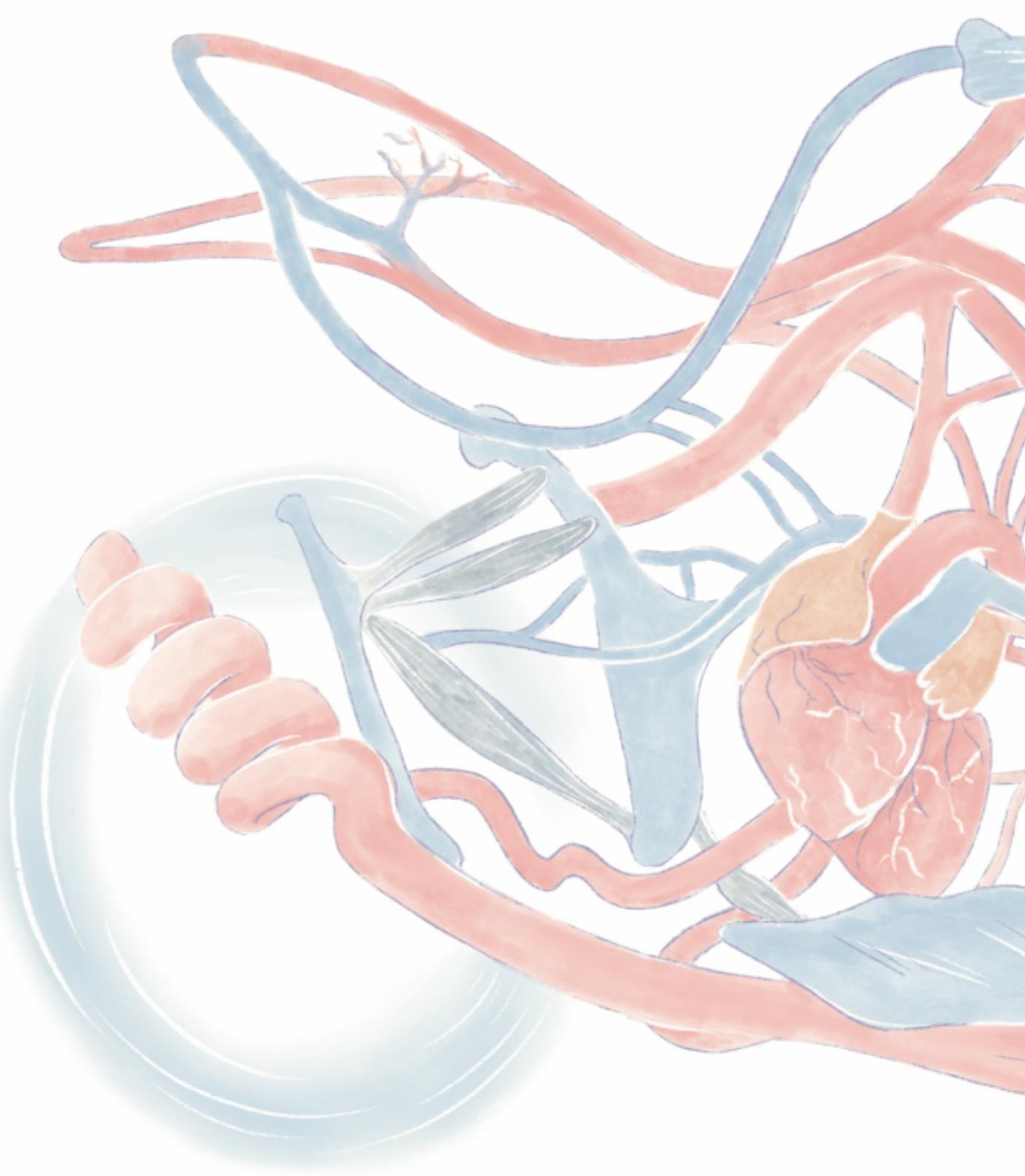




\section{Chapter 6}

Reactive oxygen-forming Nox5 links vascular smooth muscle cell phenotypic switching and extracellular vesicle-mediated-vascular calcification

Malgorzata Furmanik ${ }^{\#}$, Martijn Chatrou\#, Rick van Gorp, Asim Akbulut, Brecht

Willems, Harald Schmidt, Guillaume van Eys, Marie-Luce Bochaton-Piallat, Diane Proudfoot, Erik Biessen, Ulf Hedin, Ljubica Perisic Matic, Barend Mees, Catherine Shanahan, Chris Reutelingsperger, Leon Schurgers \# These authors contributed equally. 


\begin{abstract}
Rationale: Vascular calcification, the formation of calcium phosphate crystals in the vessel wall, is mediated by vascular smooth muscle cells (VSMCs). However, the underlying molecular mechanisms remain elusive precluding mechanism-based therapies.

Objective: Phenotypic switching denotes a loss of contractile proteins and an increase in migration and proliferation, whereby VSMCs are termed synthetic. We examined how VSMC phenotypic switching influences vascular calcification and the possible role of the uniquely calcium-dependent ROS-forming NADPH oxidase 5 (Nox5).

Methods and Results: In vitro cultures of synthetic VSMCs showed decreased expression of contractile markers CNN1, $\alpha$ SMA and SM22 $\alpha$ and an increase in synthetic marker S100A4 compared to contractile VSMCs. This was associated with increased calcification of synthetic cells in response to high extracellular $\mathrm{Ca}^{2+}$. Phenotypic switching was accompanied by increased levels of reactive oxygen species (ROS) and $\mathrm{Ca}^{2+}$-dependent Nox 5 in synthetic VSMCs. Nox5 itself regulated VSMC phenotype as siRNA knock-down of Nox5 increased contractile marker expression and decreased calcification, while overexpression of Nox 5 decreased contractile marker expression. ROS production in synthetic VSMCs was cytosolic $\mathrm{Ca}^{2+}$-dependent, in line with it being mediated by Nox5. Treatment of VSMCs with $\mathrm{Ca}^{2+}$ loaded extracellular vesicles (EVs) lead to an increase in cytosolic $\mathrm{Ca}^{2+}$. Inhibiting $\mathrm{EV}$ endocytosis with dynasore blocked the increase in cytosolic $\mathrm{Ca}^{2+}$ and $\mathrm{VSMC}$ calcification. Increased ROS production resulted in increased EV release and decreased phagocytosis by VSMCs.

Conclusions: We show here that contractile VSMCs are resistant to calcification and identify Nox 5 as a key regulator of VSMC phenotypic switching. Additionally, we describe a new mechanism of $\mathrm{Ca}^{2+}$ uptake via EVs and show that $\mathrm{Ca}^{2+}$ induces ROS production in VSMCs via Nox5. ROS production is required for release of EVs, which promote calcification. Identifying molecular pathways that control Nox5 and VSMC-derived EVs provides potential targets to modulate vascular remodelling and calcification in the context of mineral imbalance.
\end{abstract}




\section{Introduction}

Vascular calcification is the formation of calcium phosphate crystals in the blood vessel wall (1) and is associated with a 3-4-fold increase in cardiovascular and all-cause mortality (2). Vascular calcification can be categorised based on its location in the vessel wall into intimal, predominantly associated with atherosclerosis, and medial, associated with ageing, chronic kidney disease and diabetes (3). The result of intimal calcification is an increased risk of plaque rupture (4), which causes myocardial infarction and strokes (5). Medial calcification in chronic kidney disease is attributed to an imbalance in calcium and phosphate metabolism (6). The direct result of medial vascular calcification is blood vessel wall stiffening, that leads to many cardiovascular complications such as hypertension and aortic stenosis. These in turn give rise to cardiac hypertrophy, myocardial and lower-limb ischemia, congestive heart failure and can eventually result in death (7). To date, no early detection methods and no proven therapies exist to inhibit or reverse vascular calcification.

Vascular calcification is an active process regulated by vascular smooth muscle cells (VSMCs) via several mechanisms including apoptosis (8), osteo/chondrogenic transdifferentiation (9), extracellular vesicle release (10), and cellular senescence (11). In healthy arteries, most VSMCs of the tunica media maintain a contractile phenotype, which enables them to regulate vascular tone and maintain hemodynamic balance. Physiological or pathological changes may demand adaptation of the involved arteries. Stress signals, such as oxidative or mechanical stress, cause VSMC phenotype to change. These dedifferentiated or 'synthetic' VSMCs are characterized by a decreased expression of contractile proteins (myosin heavy and light chain (MLC), calponin (CNN1), smoothelin, $\alpha$-smooth muscle actin ( $\alpha$ SMA), smooth muscle protein $22 \alpha$ (SM22 $\alpha)$ ), increased proliferation and migration (12, 13). The process of dedifferentiation is termed phenotypic switching. Phenotypic switching is thought to precede the development of vascular disease. In specific pathologies VSMCs have been shown to differentiate even further and give rise to other cell types in the vessel wall including macrophages (14) and osteo/chondrogenic cells $(15,16)$.

Though phenotypic switching is thought to precede vascular disease, including vascular calcification, molecular events leading to the loss of contractile phenotype and mechanisms driving the synthetic VSMCs towards calcification, are at present not fully understood. Therefore, in this study we set out to investigate the relationship between VSMC phenotypic switching and vascular calcification and the mechanisms that link these processes.

\section{Materials and methods}

An expanded Materials and Methods is available in the Online Data Supplement.

Cell culture, treatments and transfections. Human and porcine aortic VSMCs (hVSMCs and pVSMCs) were derived from tissue explants and cultured as described previously (17, 18). Collection, storage, and use of tissue and human aortic samples were performed in 
agreement with the Dutch Code for Proper Secondary Use of Human Tissue. HASMC66 cell line was cultured as described previously (19). Each experiment was carried repeated in VSMCs from at least 3 different donors. VSMCs in passages 5-12 were used. Heparin, PDGF-BB, GKT136901, VAS2870, BAPTA-AM, dynasore and $\mathrm{H}_{2} \mathrm{O}_{2}$ were used at concentrations stated in the figure legends. For calcification assays VSMCs were treated with control $\left(1.8 \mathrm{mM} \mathrm{Ca}^{2+}\right)$ or high calcium $\left(3.6 \mathrm{mM}\right.$ or $\left.5.4 \mathrm{mM} \mathrm{CaCl}_{2}\right)$ medium. Calcification was measured as previously described (20). HVSMCs were transduced with lentiviral vectors Nox5- $\beta$ and GFP sequences for $48 \mathrm{~h}$. SiRNA (S103243856, Qiagen) transfection was carried out using a Basic Smooth Muscle Cells Nucleofector Kit (Lonza) for 24 hours.

Cell assays. PVSMC proliferation was measured real-time using the xCELLigence System (ACEA Biosciences). ROS were quantified with Amplex red or DCFDA. Cytosolic $\mathrm{Ca}^{2+}$ was measured using Fluo-4-AM in Cytation3 (Biotek). Live single cell imaging of cytosolic $\mathrm{Ca}^{2+}$ was performed by monitoring fura- 2 fluorescence using an Olympus Cell ${ }^{\wedge} \mathrm{R}$ imaging system as described previously (21).

Immunoblotting, Quantitative Reverse Transcriptase Polymerase Chain Reaction, and Immunofluorescence. VSMC RNA was reverse transcribed using Mu-MLV reverse transcriptase (Invitrogen) and the SYBR quantitative polymerase chain reaction assay (BioRad) according to manufacturer's protocol. Immunoblotting and immunofluorescence were performed as previously described (22).

The BiKE cohort. Patients undergoing surgery for symptomatic (S) or asymptomatic (AS), high-grade $(>50 \%$ NASCET) carotid stenosis at the Department of Vascular Surgery, Karolinska University Hospital, Sweden were consecutively enrolled in the study and clinical data recorded on admission (23). All samples were collected with informed consent from patients or organ donors' guardians. All human studies were approved by the regional Ethical Committees. The microarray dataset is available from Gene Expression Omnibus (GSE21545).

Immunohistochemistry. Human coronary artery sections were collected during autopsy from 20 patients aged 47 to 86 years, who died from non-cardiac causes. Autopsy was performed 6 to 9 hours after death (Department of Pathology, Academic Hospital Maastricht, Maastricht). Tissue collection was approved by the Maastricht Pathology Tissue Collection committee. The Medical Ethics Committee of the Maastricht University approved the study protocol and all subjects gave their informed consent in writing. Immunohistochemical staining was performed as described elsewhere (24).

Extracellular vesicle isolation and quantification. EVs were isolated by differential ultracentrifugation as previously described (25) from HASMC66 (26) cells, pVSMCs and hVSMCs. Quantification of EVs secreted in the cell culture media was performed using a bead capture assay, as previously described (27).

Phagocytosis of EVs and CFSE loading. EVs (15 $\mu$ g protein), collected from HASMC66 were labelled with CFSE for $30 \mathrm{~min}$ at $37^{\circ} \mathrm{C}$ and incubated with VSMCs. After incubation, cells were trypsinised, washed and measured by flow-cytometry (Acuri C6, BD biosciences). 
Statistical analysis. Data are shown as mean $\pm \mathrm{SD}$ and were obtained in three or more independent experiments. Normality of all data was tested using the Shapiro-Wilk test. If data was normally distributed, statistical significance was tested with t-test, one sample t-test and 1-way ANOVA with Bonferroni post hoc for experiments with 2 and more groups, respectively. If data was not normally distributed, the Man-Whitney and Kruskall-Wallis tests were used. The exact test used for each data set is mentioned in figure legends. Statistical analysis was carried out using using GraphPad Prism 8.2.0. * denotes $\mathrm{p}<0.05$, ** $\mathrm{p}<0.01$, $* * * \mathrm{p}<0.001$.

Pearson correlations were used to calculate the association between mRNA expression of NOX5 and other markers from microarray dataset. Significance was considered when $\mathrm{p}<0.05$.

Data availability. The data that support the findings of this study are available from the corresponding author upon reasonable request.

\section{Results}

Phenotypic switching of VSMCs in vitro is associated with calcification due to increased ROS production

Cultured VSMCs display progressive loss of contractile proteins, shifting towards a synthetic phenotype (28). In order to study the relationship between VSMC phenotype and calcification in vitro we used two model systems: 1) porcine VSMCs (pVSMCs) isolated as distinct populations of contractile or synthetic cells (18) and 2) human VSMC (hVSMCs) treated with heparin or PDGF-BB in order to induce the contractile or synthetic phenotype, respectively $(18,29)$. We confirmed that contractile pVSMCs express higher levels of contractile markers $\mathrm{CNN1}$ and $\alpha \mathrm{SMA}$ (Figure 1A) than synthetic cells. As previously described (18) the morphology of the cells differed, with synthetic cells being more rhomboid and contractile cells elongated. Next we demonstrated that the phenotype switch is reversible, as treatment of contractile pVSMC with PDGF-BB caused a loss of $\alpha$ SMA, SM22 $\alpha$ and $\mathrm{CNN} 1$ expression and a concomitant gain of S100A4 expression, a synthetic marker (Figure 1B-F) (30). Conversely, treating synthetic pVSMCs with heparin led to upregulated $\alpha$ SMA, SM22 $\alpha$ and CNN1 and decreased S100A4 expression indicating a switch towards a contractile phenotype. Similar effects of PDGF-BB and heparin were observed in hVSMCs (Supplementary Figure 1A, 1B). Additionally, synthetic VSMCs showed higher rates of proliferation (Figure 1G, Supplementary Figure 1C) than contractile VSMCs.

In order to accelerate the process of calcification in vitro we cultured VSMCs in medium with an increased $\mathrm{Ca}^{2+}$ concentration. Contractile pVSMCs showed no significant $\mathrm{Ca}^{2+}$ crystal formation, whereas synthetic pVSMCs exposed to calcifying conditions calcified significantly (Figure 2A, Supplementary Figure 1D). The increased calcification could be partly rescued by 'reversing' pVSMC phenotype with heparin (Figure 2B and 2C). This suggests that the rate of calcification is linked to VSMC phenotype. 
A

Brightfield

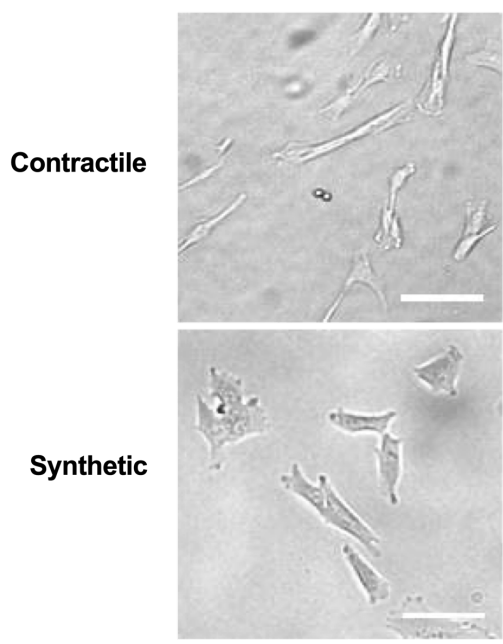

B

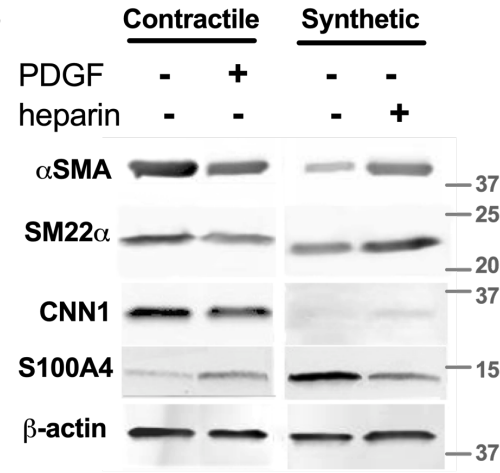

CNN1
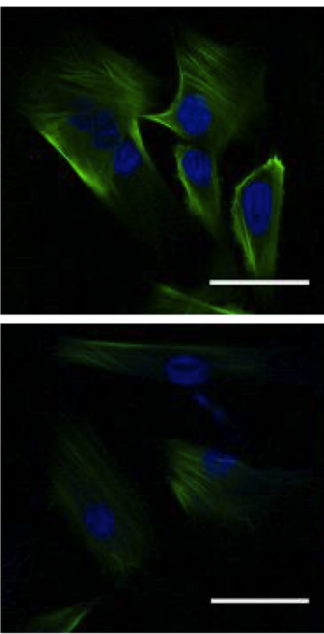

C

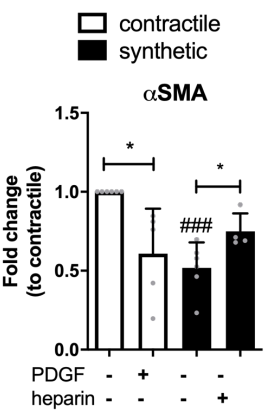

aSMA
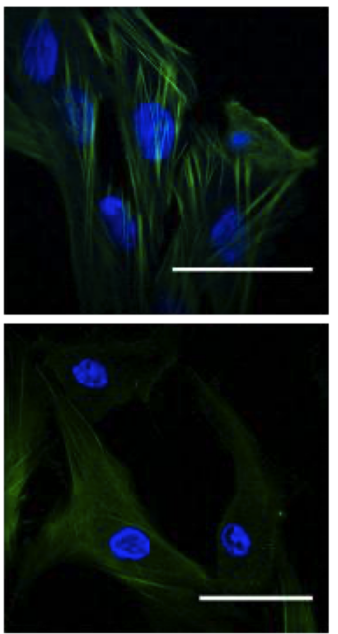

D

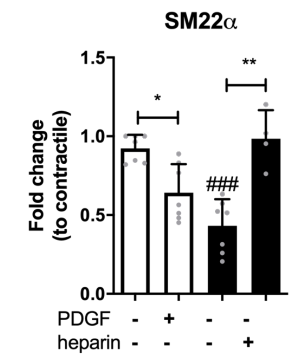

E

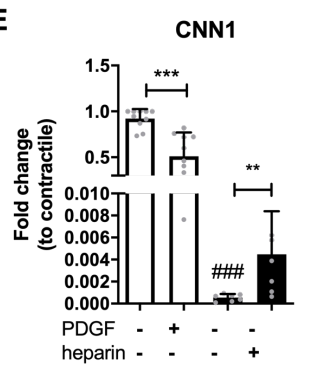

F

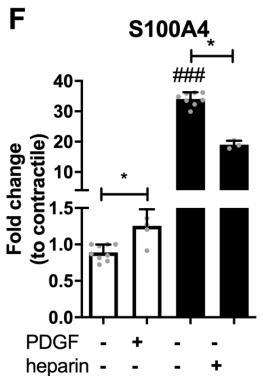

G
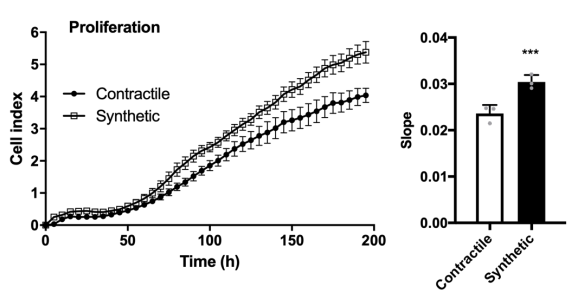

Figure 1. Phenotypic switching of pVSMCs is associated with changes in contractile gene expression and proliferation. (A) Brightfield images and immunocytochemical staining of contractile proteins in synthetic and contractile pVSMCs showing differences in cell morphology and CNN1 and $\alpha$ SMA expression. Scale bars: $50 \mu \mathrm{m}$ (immunocytochemistry), $1 \mathrm{~mm}$ (brightfield). Representative images from 3 independent experiments. (B-F) Western blotting and quantification of contractile proteins and synthetic marker in contractile cells treated with PDGF and synthetic cells treated with heparin, showing phenotypic switching was partially reversible. Cells were 
grown in DMEM with $10 \%$ FBS with $20 \mathrm{ng} / \mathrm{ml}$ PDGF for 2 days or $200 \mathrm{U} / \mathrm{ml}$ heparin for 5 days. Graphs show pooled data from 3 independent experiments. Statistical significance was tested using the Mann-Whitney test and one sample t-test (C-F) or t-test (F). (G) Proliferation of pVSMCs was measured using the xCELLigence system, which measures the impedance of cells adhering to an electrode-covered surface, $n=3$. Synthetic cells show increased proliferation. Statistical significance was tested using t- test.

A similar dependency of calcification on phenotype was observed in hVSMCs (Supplementary Figure 1 E, F).

We next set out to investigate the mechanisms, which could explain the different rates at which contractile and synthetic VSMCs calcify. Expression of osteogenic genes, classically associated with calcification, was not different between the phenotypes at baseline, except a higher expression of MGP mRNA in contractile pVSMCs compared to synthetic pVSMCs (Supplementary Figure 1G, 1H). Therefore, we focused on oxidative stress, which is a known mediator of vascular calcification (31). Contractile and synthetic pVSMCs produced comparable amounts of $\mathrm{H}_{2} \mathrm{O}_{2}$ under non-calcifying conditions (Figure 2D). However, exposure to high $\mathrm{Ca}^{2+}$ levels resulted in increased production of $\mathrm{H}_{2} \mathrm{O}_{2}$ by synthetic pVSMCs (Figure 2D). This suggested that in these cells the rise in ROS production is $\mathrm{Ca}^{2+}$-dependent. In support of this notion, antioxidant $\mathrm{N}$-acetylcysteine (NAC) was able to decrease $\mathrm{Ca}^{2+}$-induced calcification of synthetic pVSMCs (Figure 2E).

In order to examine the mechanism responsible for increased ROS in synthetic VSMCs, we compared expression of three NADPH oxidase (Nox) enzymes between the phenotypes. We found that Nox1 expression was similar in both phenotypes, but both Nox 4 and Nox 5 were significantly upregulated in synthetic pVSMCs compared to contractile pVSMCs both on mRNA and protein level (Figure 2F-J, Supplementary Figure 1I).

\section{Nox5 is highly expressed in synthetic VSMCs and mediates phenotypic switching and calcification}

We next set out to investigate whether Nox4 and Nox5 were involved in synthetic phenotype-related calcification in our model. Since Nox4 was expressed at lower levels than Nox5 and we excluded a role for Nox4 in VSMC calcification (Supplementary figure 2A), we focused on Nox5. First, we confirmed that switching contractile pVSMCs to synthetic cells with PDGF significantly increased expression of Nox5 mRNA (Figure 3A), whereas switching synthetic pVSMCs with heparin to contractile cells decreased Nox5 levels (Figure 3B). Knock-down of Nox5 in hVSMCs resulted in increased expression of contractile markers CNN1, p-MLC and SM22 $\alpha$ (Figure 3C-3G) and decreased calcification (Figure 3H). Conversely, overexpression of Nox 5 resulted in decreased expression of contractile markers p-MLC and CNN1 as well as synthetic marker S100A4 (Figure 3I-3M, Supplementary Figure 2B) and increased calcification (Figure $3 \mathrm{~N}$ and 3O). Additionally, $\mathrm{H}_{2} \mathrm{O}_{2}$ treatment decreased p-MLC and S100A4 expression in hVSMCs (Supplementary Figure 2C). These results imply that signals which induce phenotype changes, do so via decreased or increased expression of Nox 5 and resulting changes in ROS levels. 


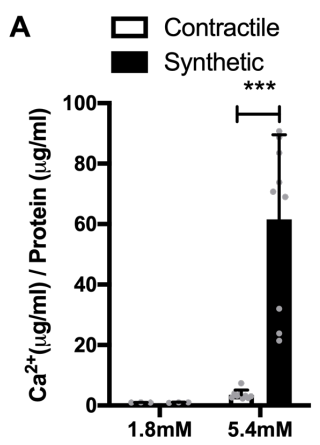

D

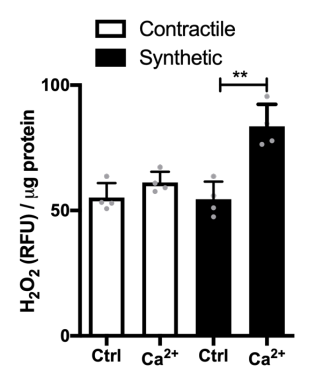

G

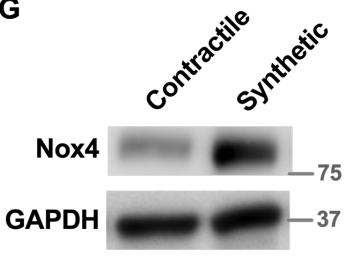

B

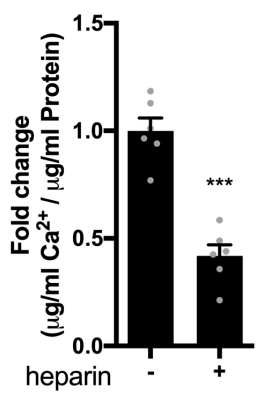

E

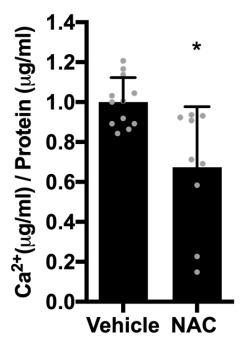

C

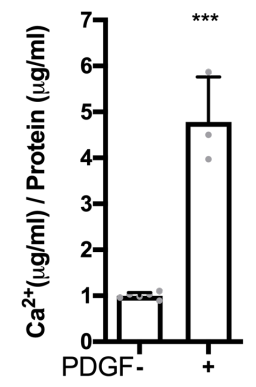

F Quantitative real-time PCR

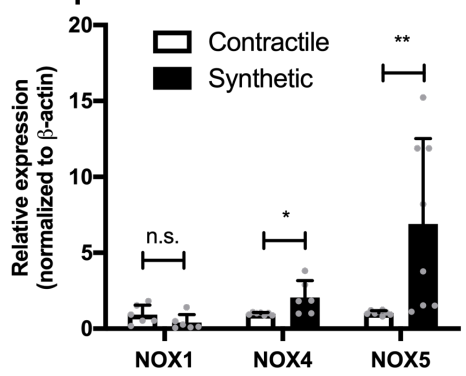

I
H

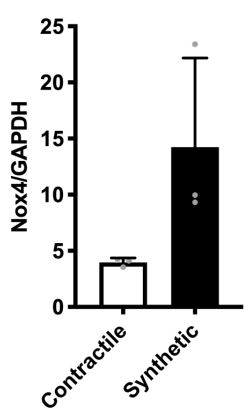

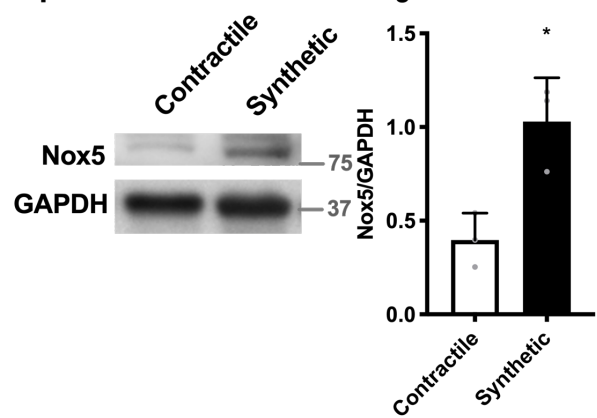

Figure 2. Synthetic phenotype is associated with increased calcification, ROS production and Nox expression. Calcification was induced by incubation with elevated $\mathrm{Ca}^{2+}$ concentrations $(5.4 \mathrm{mM})$ in DMEM with $0.5 \% \mathrm{FBS}$ and quantified using an o-cresolphthalein colorimetric assay. (A) Synthetic pVSMCs showed significant calcification in contrast to contractile cells after $48 \mathrm{~h}$. Statistical significance was tested using the Mann-Whitney test. (B) Treating synthetic pVSMCs with heparin decreased calcification after $48 \mathrm{~h}$. Statistical significance was tested with t-test. (C) Treating contractile pVSMCs with PDGF for $48 \mathrm{~h}$ increased calcification. Statistical significance was tested with ttest. (D) Synthetic pVSMCs treated with $5.4 \mathrm{mM} \mathrm{Ca}^{2+}$ in $0.5 \% \mathrm{FBS}$ for $22 \mathrm{~h}$ produced more $\mathrm{H}_{2} \mathrm{O}_{2}$ than contractile cells. $\mathrm{H}_{2} \mathrm{O}_{2}$ production was measured using Amplex red. Statistical significance was tested with $\mathrm{t}$-test. (E) Scavenging $\mathrm{H}_{2} \mathrm{O}_{2}$ with $1 \mathrm{mM} \mathrm{N}$-acetylcysteine (NAC) decreased calcification of synthetic pVSMCs treated with 5.4 $\mathrm{mM} \mathrm{Ca}^{2+}$ in $0.5 \% \mathrm{FBS}$ for $48 \mathrm{~h}$. Statistical significance was tested using the Mann-Whitney test. (F) QPCR analysis showed increased Nox4 and Nox 5 expression in synthetic pVSMCs at baseline. Statistical significance was tested with t-tests. (G-J) Western blotting and quantification of Nox4 and Nox5 in contractile and synthetic pVSMCs. 
Statistical significance was tested with t-tests. Data from a representative experiment. All other graphs show data from 3 independent experiments.

A

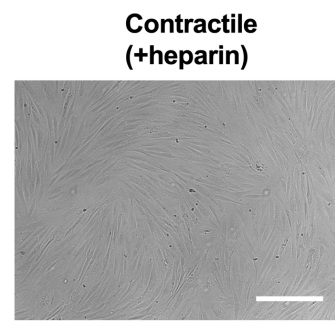

Synthetic (+PDGF-BB)

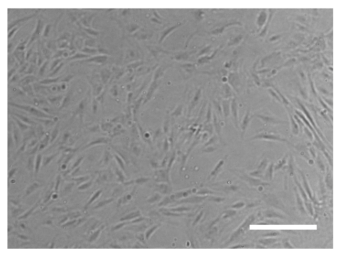

B

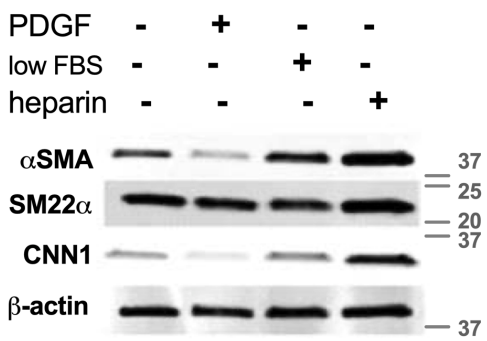

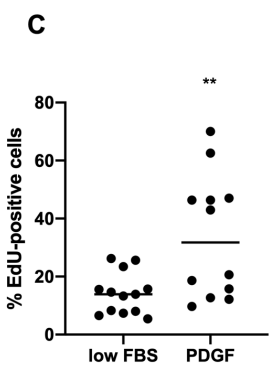

D

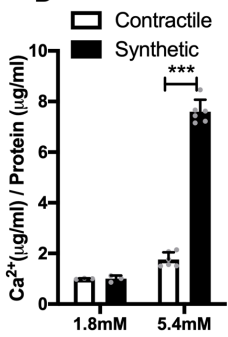

E

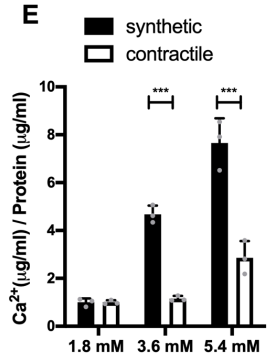

F $\square$ synthetic

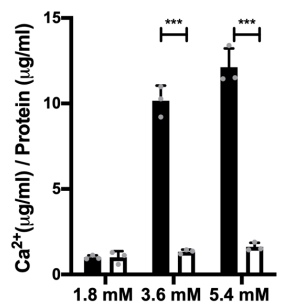

G
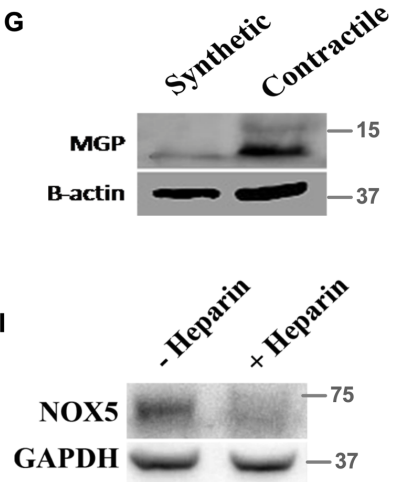

H

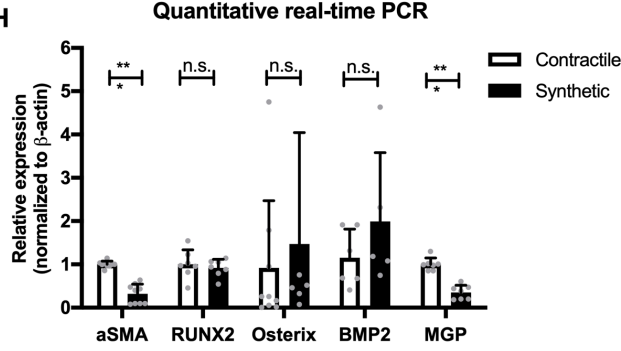

Supplementary Figure 1. Phenotypic switching. VSMCs were grown in DMEM with 10\% FBS with $20 \mathrm{ng} / \mathrm{ml}$ PDGF for 2 days to induce synthetic differentiation or $200 \mathrm{U} / \mathrm{ml}$ heparin for 5 days to induce contractile differentiation. Calcification was induced by incubation with elevated $\mathrm{Ca}^{2+}$ concentrations $(5.4 \mathrm{mM})$ in DMEM with $0.5 \%$ FBS and quantified using an o-cresolphthalein colorimetric assay. (A) Brightfield images of synthetic and contractile hVSMCs showing differences in cell morphology. Scale bars are $10 \mathrm{~mm}$. (B) Western blotting analysis of contractile proteins and in hVSMCs. PDGF induced hallmarks of a synthetic phenotype with decreased contractile markers. Low FBS treatment ( $0.5 \%$ for 5 days) had a similar effect to heparin, increasing expression of contractile markers. Representative images from 2 independent experiments. (C) Synthetic hVSMCs (treated with PDGF) proliferate at a higher rate than contractile hVSMCs (exposed to $0.5 \%$ FBS) in a period of 24 hours, measured by EdU incorporation. Statistical significance was tested using t-test. Pooled data from 4 experiments. (D) Synthetic pVSMCs calcified in $5.4 \mathrm{mM} \mathrm{Ca}^{2+}$ in medium with $2.5 \% \mathrm{FBS}$ after $72 \mathrm{~h}$. Statistical significance was tested using ttests. (E, F) Synthetic hVSMCs calcified more that contractile hVMSCs both in medium supplemented with (E) 
$0.5 \%$ FBS after $72 \mathrm{~h}$ and (F) $2.5 \%$ FBS after 5 days. Statistical significance was tested using t-tests. (G) Contractile pVSMCs expressed increased MGP protein levels, $n=1$. (H) Expression on bone markers wan not significantly different between contractile and synthetic pVSMCs, except for MGP. Statistical significance was tested using ttests (Runx2, BMP2, MGP) or Mann-Whitney tests (Osterix and aSMA) (I) Western blot of hVSMCs showing that heparin (200 U/ml, 5 days) decreased Nox5 expression, $\mathrm{n}=1$. All graphs show representative data from 3 independent experiments unless stated otherwise.

Interestingly, our results suggest that upregulation of S100A4 in synthetic cells occurs via mechanisms other that Nox5-mediated ROS upregulation.

\section{$\mathrm{Ca}^{2+}$-dependent oxidative stress mediates pVSMC calcification}

Nox5 is known to be activated by binding of cytosolic $\mathrm{Ca}^{2+}$ to its EF-hand (32), therefore we hypothesized that it is the molecular link between increased extracellular $\mathrm{Ca}^{2+}$, and ROS-dependent calcification of synthetic VSMCs. First, we investigated extracellular vesicle (EV)-mediated uptake as a possible mechanism by which increased extracellular $\mathrm{Ca}^{2+}$ can increase cytosolic $\mathrm{Ca}^{2+}$. To this end hVSMCs were treated with dynasore, an inhibitor of clathrin- and caveolin-dependent EV uptake (33) in the presence of high $\mathrm{Ca}^{2+}$. Dynasore blocked the increase in cytosolic $\mathrm{Ca}^{2+}$ caused by the high $\mathrm{Ca}^{2+}$ treatment (Figure 4A), suggesting that $\mathrm{Ca}^{2+}$ enters the cells via EVs. Ionomycin, which causes a rapid influx of $\mathrm{Ca}^{2+}$ into the cells, was used as a positive control. In line with this, EVs isolated from cells incubated with $3.6 \mathrm{mM} \mathrm{Ca}^{2+}$ accumulated significantly higher $\mathrm{Ca}^{2+}$ compared to EVs isolated from cells treated with normal calcium $\left(1.8 \mathrm{mM} \mathrm{Ca}^{2+}\right.$; Figure 4B). When EVs were isolated from cells treated in normal $\mathrm{Ca}^{2+}$, but later incubated with high $\mathrm{Ca}^{2+}$ in the absence of cells, they also accumulated $\mathrm{Ca}^{2+}$ (Figure $4 \mathrm{C}$ ) suggesting that $\mathrm{Ca}^{2+}$ loading into EVs happens outside of the cells. Additionally, we carried out a more sensitive cytosolic $\mathrm{Ca}^{2+}$ assay in single cells. VSMCs were treated with high $\mathrm{Ca}^{2+}$, which resulted in an insignificant increase in cytosolic $\mathrm{Ca}^{2+}$ in 1 hour, compared to control (Figure 4D). Interestingly, when VSMCs cultured in medium with normal $\mathrm{Ca}^{2+}$ were treated with EVs isolated from VSMCs treated with high $\mathrm{Ca}^{2+}$, transient increases in cytosolic $\mathrm{Ca}^{2+}$ were observed (Figure 4E). In VSMCs treated with EVs isolated from VSMCs incubated with normal $\mathrm{Ca}^{2+}$ no such increases were observed. These observations suggest that EVs generated in a high $\mathrm{Ca}^{2+}$ environment induce a cytosolic $\mathrm{Ca}^{2+}$ rise in VSMCs. Additionally, dynasore inhibited calcification of VSMCs (Figure 4F). This suggests that $\mathrm{Ca}^{2+}$ entry via EVs contributes to VSMC calcification. Next, we set out to investigate whether this rise of cytosolic $\mathrm{Ca}^{2+}$ leads to increased ROS production and calcification. First, we confirmed that overexpression of Nox 5 in hVSMCs, which induced synthetic differentiation, lead to increased ROS production (Figure 4G) and that siRNA knock-down of Nox5 lead to decreased ROS production (Figure 4H).

Then, we demonstrated that cytosolic $\mathrm{Ca}^{2+}$ chelator BAPTA-AM blocked the increase in $\mathrm{H}_{2} \mathrm{O}_{2}$ production by synthetic $\mathrm{pVSMCs}$ (Figure $4 \mathrm{I}$ ). $\mathrm{H}_{2} \mathrm{O}_{2}$ production was also blocked by the Nox inhibitor GKT136901 (Figure 4H). Moreover, Nox inhibitors GKT136901 and VAS2870 as well as BAPTA-AM decreased VSMC calcification (Figure 4J and 4K, 
A

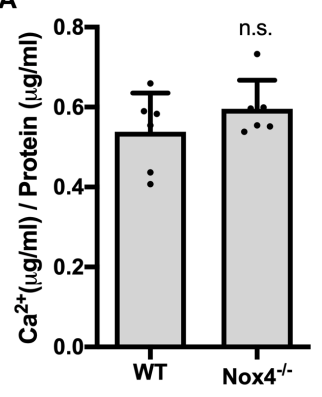

B

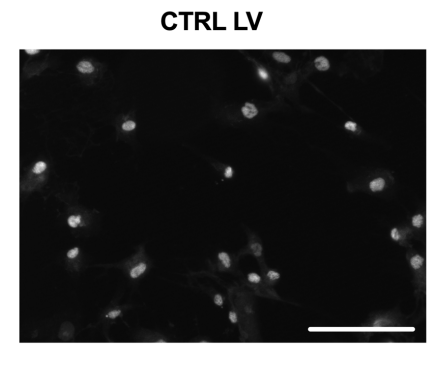

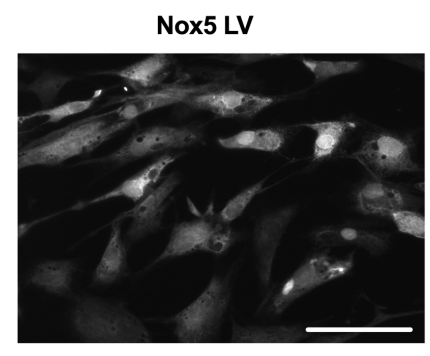

C

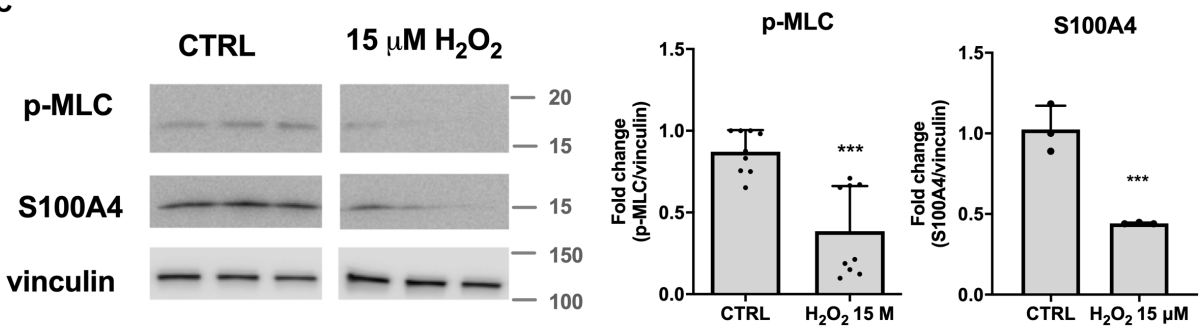

D

E
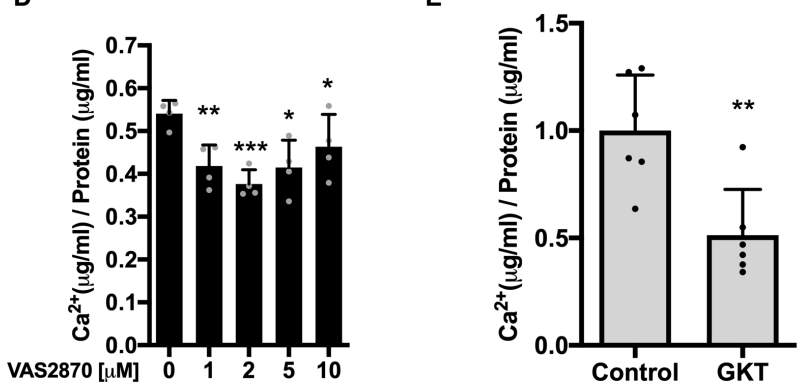

Supplementary Figure 2. Nox5 - experimental controls. (A) There was no difference in calcification between $\mathrm{Nox}^{-/ /}$and WT mouse VSMCs treated with $5.4 \mathrm{mM} \mathrm{Ca}^{2+}$ in $0.5 \% \mathrm{FBS}$ for $48 \mathrm{~h}$. Statistical significance was tested using the Mann-Whitney test. (B) Transduction of hVSMCs with a Nox 5 overexpressing virus results in increased Nox 5 expression. HVSMCs were transduced with an empty lentiviral vector or Nox 5 vector at multiplicity of infection 10 for $48 \mathrm{~h}$. Afterwards cells were fixed, stained with anti-Nox 5 antibody and fluorophore-labelled secondary antibody. Images were acquired using Cytation 3. Scale bars are $100 \mu \mathrm{m}$. (C) Western blotting and quantification of p-MLC and S100A4 in hVSMCs treated with $15 \mu \mathrm{M} \mathrm{H}_{2} \mathrm{O}_{2}$ in M199 with $2.5 \%$ FBS 5 days. Statistical significance was testes using the Mann-Whitney test (p-MLC) and t-test (S100A4). (D) Nox inhibitor VAS2870 decreased calcification of synthetic pVSMCs treated with $5.4 \mathrm{mM} \mathrm{Ca}^{2+}$ in $0.5 \% \mathrm{FBS}$ for $48 \mathrm{~h}$. Statistical significance was tested using ANOVA. (E) Nox inhibitor GKT136901 $(10 \mu \mathrm{M})$ inhibited calcification of hVSMCs. Calcification was induced by incubation $5.4 \mathrm{mM} \mathrm{Ca}^{2+}$ in $2.5 \% \mathrm{FBS}$ for $72 \mathrm{~h}$. Statistical significance was tested using t-test. All graphs show representative data from 3 independent experiments depicted as mean $\pm \mathrm{SD}$. ${ }^{*} \mathrm{P}<0.05$, ${ }^{*} * \mathrm{P}<0.01,{ }^{* * *} \mathrm{P}<0.001$. All graphs show representative data from 3 independent experiments.

Supplementary Figure 2D, 2E). Taken together these results show that cytosolic $\mathrm{Ca}^{2+}$-Nox5dependent ROS production is required for calcification of synthetic VSMCs. 
A

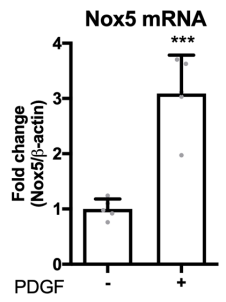

E

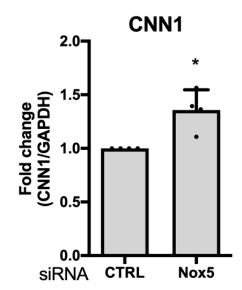

I
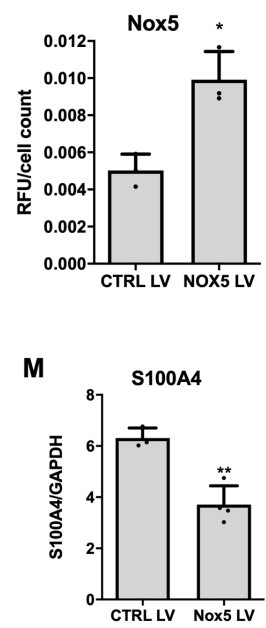

B

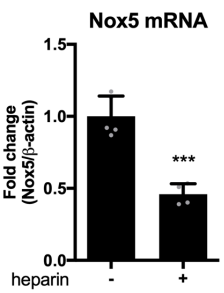

F

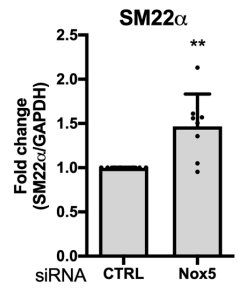

J

LV CTRL Nox5

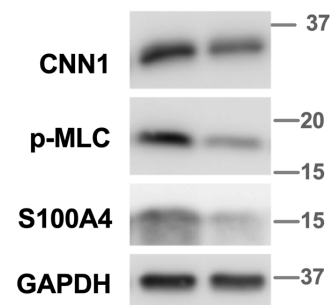

$\mathbf{N}$

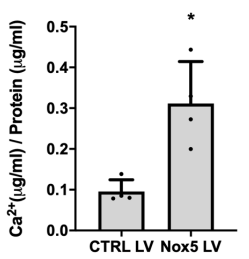

C
SIRNA CTRL Nox5

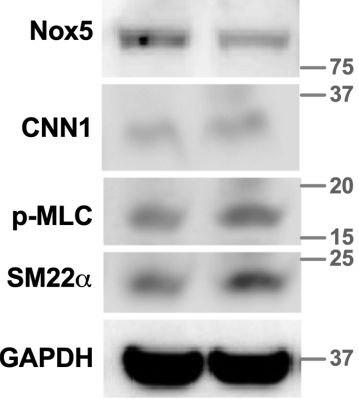

G

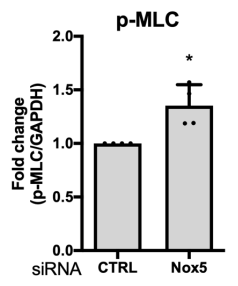

K

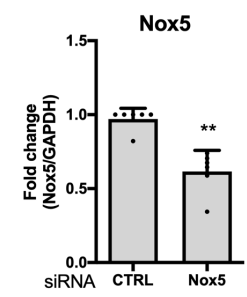

H

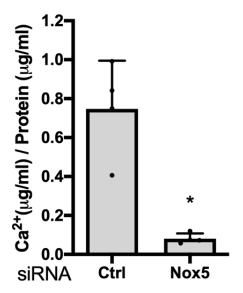

L
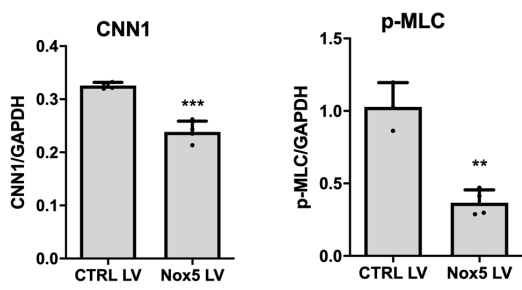

O

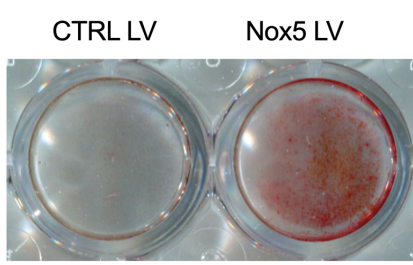

Figure 3. Nox5 mediates phenotypic switching and VSMC calcification. (A, B) qPCR analysis of Nox 5 mRNA expression in synthetic pVSMCs treated with heparin $(200 \mathrm{U} / \mathrm{ml}, 5$ days $)$ and contractile pVSMCs treated with PDGF (20 ng/ml, 2 days). Expression was normalised to $\beta$-actin. (C-G) SiRNA knock-down (24 h) of Nox5 increased expression of contractile markers in hVSMCs (western blot and quantifications), $\mathrm{n}=3$. Statistical significance was tested using the Mann-Whitney test (D, F) or one sample t-test (E, G). (H) SiRNA knock-down ( $24 \mathrm{~h}$ ) of Nox 5 decreased calcification of hVSMCs (calcification was induced with $5.4 \mathrm{mM} \mathrm{Ca}^{2+}$ in $2.5 \%$ FBS for 5 days). Statistical significance was tested using t-test. (I-M) Nox5 overexpression using a lentivirus resulted in decreased contractile protein expression and decreased S100A4 expression in hVSMCs (Western blotting and 
quantification). Nox 5 was quantified using immunocytochemistry on the Cytation 3 imaging reader. Statistical significance was tested using t-tests. $(\mathbf{N}, \mathbf{O})$ Nox 5 overexpression increased calcification of hVSMCs treated with $5.4 \mathrm{mM} \mathrm{Ca}^{2+}$ in $2.5 \%$ FBS for 3 days, measured using o-cersolphthtalein and visualised with Alizarin Red $\mathrm{S}$. Statistical significance was tested using the Mann-Whitney test. All graphs show representative data from 3 independent experiments.

A

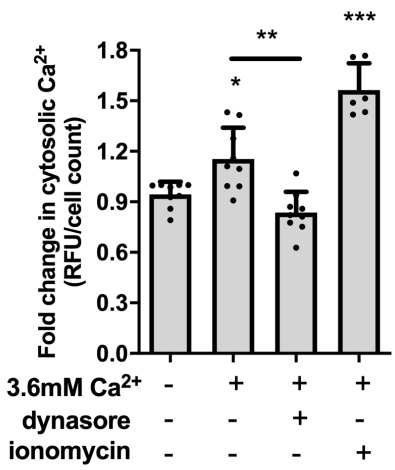

D
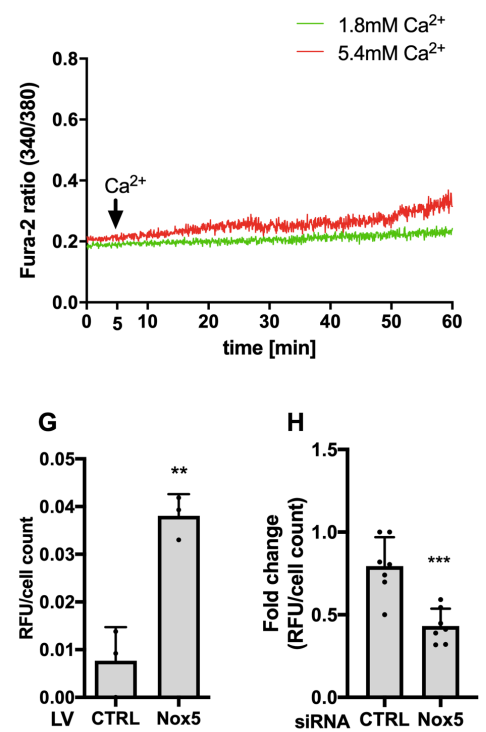

B

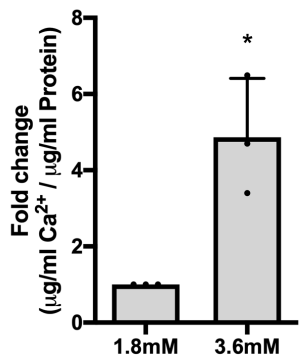

E
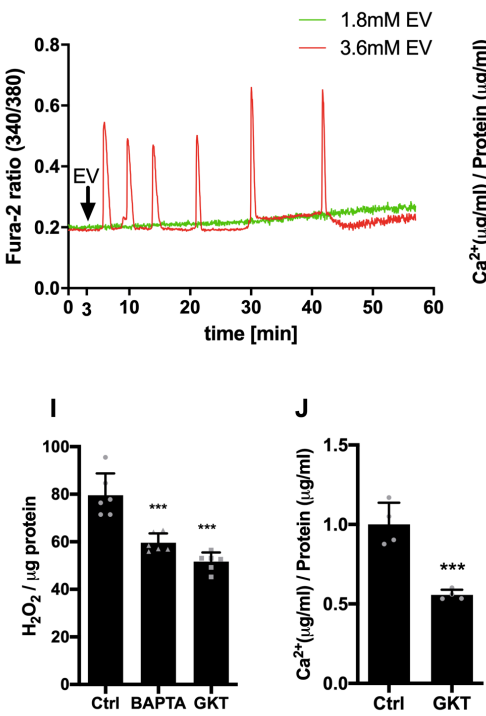

C

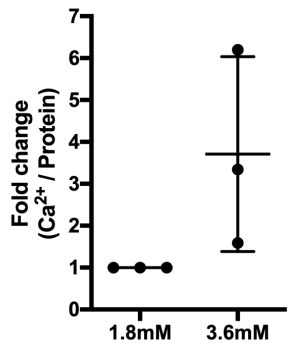

$\mathbf{F}$
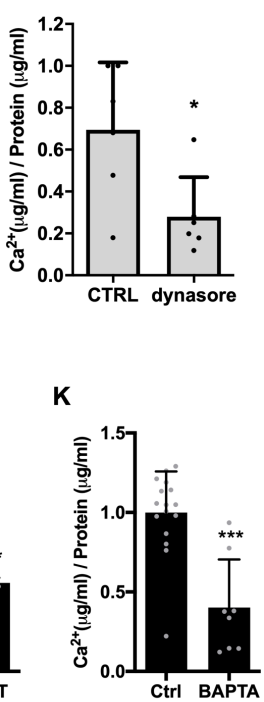

Figure 4. $\mathrm{Ca}^{2+}$-dependent oxidative stress mediates calcification of synthetic VSMCs. (A) HVSMCs were treated with $3.6 \mathrm{mM} \mathrm{Ca}^{2+}, 40 \mu \mathrm{M}$ dynasore or $1 \mu \mathrm{M}$ ionomycin for $4 \mathrm{~h}$ in $\mathrm{M} 199$ with $0.5 \% \mathrm{FBS}$. Cytosolic $\mathrm{Ca}^{2+}$ was measured using Fluo-4-AM in Cytation 3 imager. Graph shows pooled data from 3 independent experiments. Statistical significance was tested using the Kruskall-Wallis test. (B) HVSMCs were treated with M199 with 2.5\% FBS with normal $(1.8 \mathrm{mM})$ or high $(3.6 \mathrm{mM}) \mathrm{Ca}^{2+}$ for $48 \mathrm{~h}$. No calcification was observed. EVs were isolated by ultracentrifugation, $\mathrm{Ca}^{2+}$ content in $\mathrm{EVs}$ was quantified using an o-cresolphthalein colorimetric assay and normalised to protein content. Pooled data from 3 experiments, significance tested using one sample t-test. (C) Medium was collected from $10^{6} \mathrm{hVSMCs}$ grown in M199 with 20\% FBS and incubated with CD63-coupled beads overnight to capture EVs. Next, the EV bead pool was aliquoted and aliquots were treated with $3.6 \mathrm{mM} \mathrm{Ca}^{2+}$ or normal $\mathrm{Ca}^{2+}$, washed and $\mathrm{Ca}^{2+}$ content was quantified using an o-cresolphthalein colorimetric assay. Graph shows pooled data 
from 3 experiments. Statistical significance was tested using one sample t-test. (D) HVSMCs were loaded with Fura-2-AM which was alternately excited at $340 \mathrm{~nm}$ and $380 \mathrm{~nm}$ and the ratiometric emission, corresponding to cytosolic $\mathrm{Ca}^{2+}$ concentration, is presented. Cells were treated with normal $(1.8 \mathrm{mM})$ or high $(5.4 \mathrm{mM})$ extracellular $\mathrm{Ca}^{2+}$ after 5 minutes of live monitoring and continued monitoring up to 60 minutes. Results shown are responses for 2 individual cells, representing approximately 20 cells from 2 independent experiments. (E) As for D. but HVSMCs were treated with EVs isolated by ultracentrifugation from HVSMCs treated for $48 \mathrm{~h}$ with medium containing 1.8 or $3.6 \mathrm{mM} \mathrm{Ca}^{2+}$. Some cells showed clear oscillatory cytosolic $\mathrm{Ca}^{2+}$ responses to EVs isolated from $3.6 \mathrm{mM} \mathrm{Ca}^{2+}$ conditions but no cells showed responses to EV from $1.8 \mathrm{mM} \mathrm{Ca}^{2+}$ conditions. Results shown are responses for 2 individual cells, representing approximately 15 cells from 2 independent experiments. (F) Inhibiting EV uptake with dynasore decreased calcification. HVSMCs were treated in DMEM with $0.5 \% \mathrm{FBS}$ and $3.6 \mathrm{mM} \mathrm{Ca}^{2+}$ with or without $8 \mu \mathrm{M}$ dynasore for $48 \mathrm{~h}$. Representative data from 3 experiments. Statistical significance was tested using the MannWhitney test. (G) Overexpression of Nox 5 induced an increase in cytosolic ROS measured using the DCFDA probe. Statistical significance was tested using t-test. (H) Nox5 knock-down decreased ROS production in hVSMCs. Pooled data from 2 experiments. Statistical significance was tested using t-test. (I) Both Nox inhibitor GKT136901 $(10 \mu \mathrm{M})$ and cytosolic $\mathrm{Ca}^{2+}$ chelator BAPTA $(2 \mu \mathrm{M})$ decreased $\mathrm{H}_{2} \mathrm{O}_{2}$ production measured with Amplex red in synthetic cells treated with $5.4 \mathrm{mM} \mathrm{Ca}^{2+}$ in $0.5 \%$ FBS. Statistical significance was tested using the Mann-Whitney test. (J, K) GKT136901 $(10 \mu \mathrm{M})$ and BAPTA $(2 \mu \mathrm{M})$ decreased calcification of synthetic pVSMCs treated with 5.4 $\mathrm{mM} \mathrm{Ca}^{2+}$ in $0.5 \% \mathrm{FBS}$ for $48 \mathrm{~h}$ and $36 \mathrm{~h}$, respectively. Statistical significance was tested using t-test $(\mathrm{J})$ and MannWhitney test (K). All graphs show representative data from 3 independent experiments, unless stated otherwise.

\section{Nox5-mediated oxidative stress induces extracellular vesicle release and inhibits phagocytosis in synthetic VSMCs}

EVs have recently been shown to play an important role in VSMC calcification (27). Therefore, we set out to investigate whether EVs could be the mediators of $\mathrm{Ca}^{2+}$-Nox5-ROSinduced calcification of synthetic VSMCs.

We first confirmed that externally added EVs (Supplementary Figure 3A) increased calcification of a collagen matrix (Figure 5A) and of synthetic (Figure 5B) and contractile pVSMCs (Supplementary Figure 3B). This was further confirmed in a Boyden chamber experiment with $\mathrm{pVSMC}$ in the upper chamber and a collagen coating in the lower chamber. Synthetic pVSMCs in the upper chamber caused significantly more calcification in the lower chamber as compared to contractile VSMCs (Supplementary Figure 3C). Quantification of EVs using a bead capture assay showed that synthetic hVSMCs released significantly more EVs than contractile hVSMCs (Figure 5C). These results suggest that synthetic VSMCs secrete more EVs, which leads to increased calcification.

We next showed that high extracellular $\mathrm{Ca}^{2+}$ levels (which lead to increased ROS production in synthetic cells, Figure 2D) induced EV release (Figure 5D). Additionally, $\mathrm{H}_{2} \mathrm{O}_{2}$ dose-dependently enhanced EV release (Figure 5E). To investigate whether EV release is mediated by Nox5, we treated hVSMCs with pan-Nox inhibitor GKT136901 and observed decreased EV release in the presence of $\mathrm{Ca}^{2+}$ (Figure 5F, Supplementary Figure 3D). Conversely, overexpression of Nox 5 in hVSMCs, which leads to increased ROS production (Figure 4G), induced a five-fold increase in EV release (Figure 5G).

Extracellular levels of EVs are the result of a balance between secretion and uptake and we showed that ROS induced secretion of EVs. 
A

Size Distribution by Number

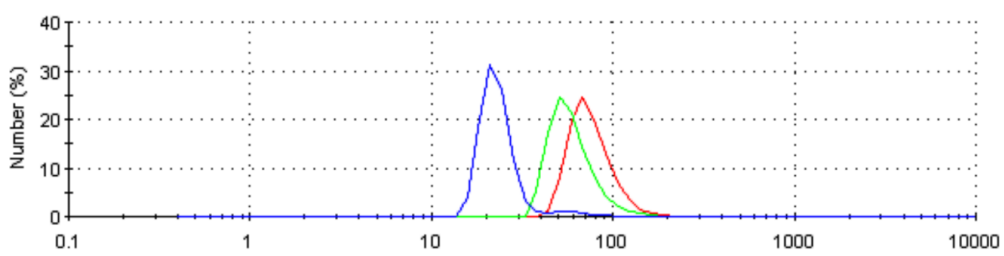

B

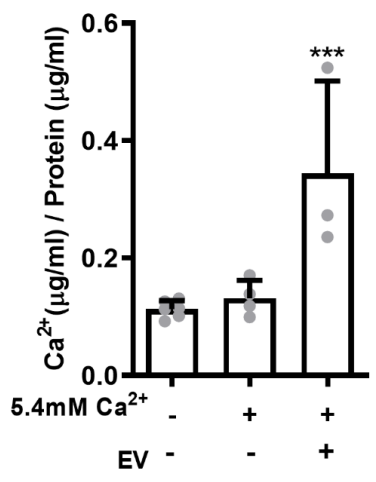

E

PS and CD81 expression on CD63-positive EVs

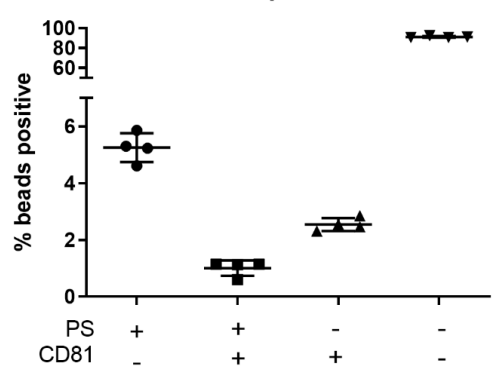

C

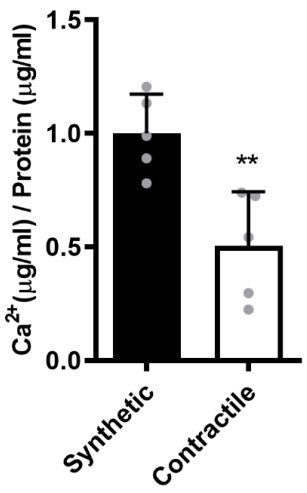

D

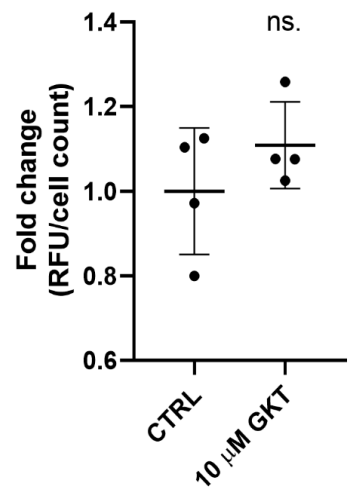

F

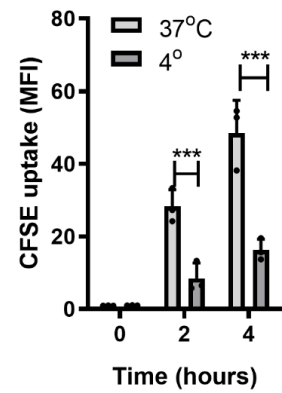

Supplementary Figure 3. Extracellular vesicles - experimental controls. (A) EVs were isolated by ultracentrifugation from culture media collected from synthetic pVSMCs grown in normal medium. Vesicles were resuspended in PBS and subjected to DLS analysis. 3 independent samples were measured, each in triplicate. Graph is a representation of a single measurement for 1 sample. Curves represent percentages of EVs of a certain size. The size of the EVs is consistent with exosomes $(10-100 \mathrm{~nm})$. (B) EVs isolated from HASMC66 (15 $\mu \mathrm{g} / \mathrm{ml}) \mathrm{were}$ added to contractile pVSMCs (pre-treated with $200 \mathrm{U} / \mathrm{ml}$ heparin for 5 days). Calcification was induced by incubation with elevated $\mathrm{Ca}^{2+}$ concentrations $(5.4 \mathrm{mM})$ in DMEM with $0.5 \%$ FBS for $24 \mathrm{~h}$ and quantified using an ocresolphthalein colorimetric assay. Statistical significance was tested using ANOVA. (C) PVSMCs were placed in the upper chamber of a Boyden chamber ( $3 \mu \mathrm{m}$ pore size) and a collagen coating in the lower chamber. Calcification of the collagen coating was induced by incubation with $5.4 \mathrm{mM} \mathrm{Ca}^{2+}$ in $0.5 \%$ FBS for $24 \mathrm{~h}$. Synthetic pVSMCs in the upper chamber caused significantly more calcification in the lower chamber compared to contractile pVSMCs. Statistical significance was tested using t-test. (D) Nox inhibitor GKT136901 (10 $\mu \mathrm{M})$ does not change EV hVSMCs 


\section{Chapter 6}

release by on its own (2.5\% FBS, $24 \mathrm{~h}$ ). Control cells were treated with vehicle (DMSO) Statistical significance was tested using t-test. (E) CD63-positive extracellular vesicles present phosphatidylserine (PS). hVSMCs were incubated in M199 with 0.5\% FBA for 16 hours, medium was changed and collected for the assay $24 \mathrm{~h}$ later. EVs were captured with anti-CD63-coulped beads and detected with an anti-CD81-PE antibody and Annexin A5-FITC, and quantified using flow cytometry. (F) CFSE-labelled EVs $(15 \mu \mathrm{g} / \mathrm{ml})$ from HASMC66 were added to hVSMCs. Uptake was quantified after 0,2 , and $4 \mathrm{~h}$ by flow cytometry. Passive uptake was monitored by incubating at $4{ }^{\circ} \mathrm{C}$. Statistical significance was tested using t-test. All graphs show representative data from 3 independent experiments.

A

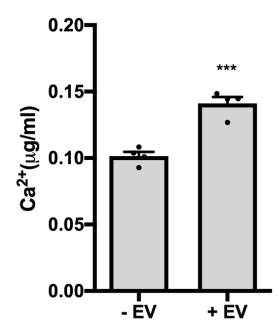

E

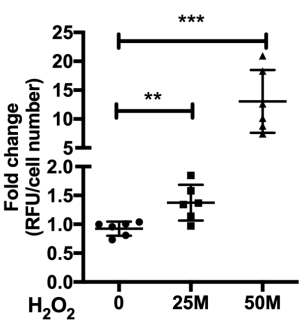

B

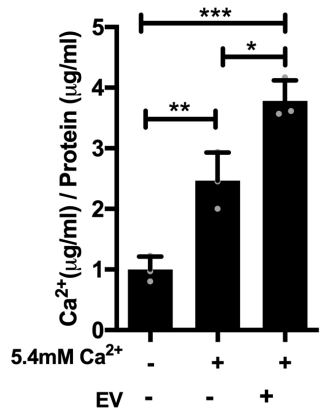

$\mathbf{F}$

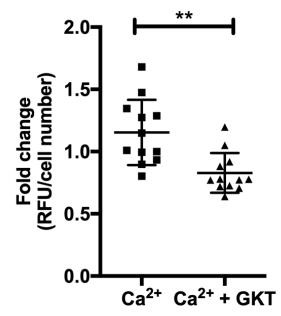

C

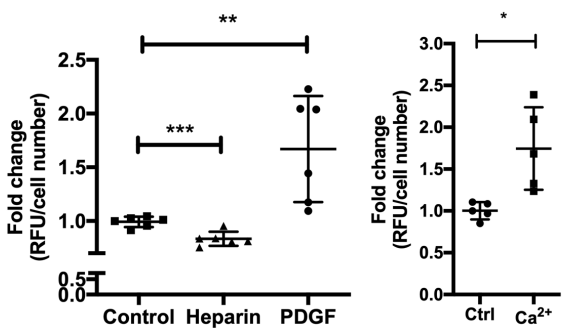

G

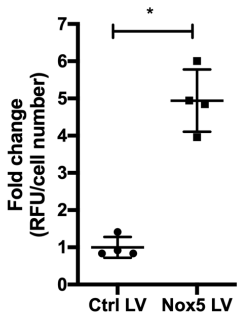

H

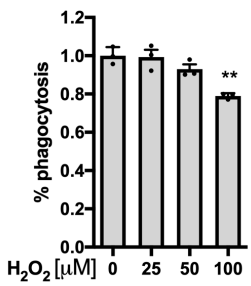

Figure 5. Nox5-dependent oxidative stress increases EV release and inhibits phagocytosis. (A) EVs isolated by ultracentrifugation from HASMC66 cultured in normal medium $(7.5 \mu \mathrm{g} / \mathrm{ml}$ protein concentration) increased calcification of a collagen matrix in the absence of cells (calcification was induced with $5.4 \mathrm{mM} \mathrm{Ca}^{2+}$ in $0.5 \%$ of EV free FBS for $24 \mathrm{~h}$ ). Statistical significance was tested using t-test. (B) EVs isolated from HASMC66 cells $(15 \mu \mathrm{g} / \mathrm{ml})$ increased calcification of synthetic pVSMCs. Statistical significance was tested using ANOVA. (C) Contractile hVSMCs (treated with $200 \mathrm{U} / \mathrm{ml}$ heparin for 5 days) secreted fewer EVs than synthetic hVSMCs (treated with 20 $\mathrm{ng} / \mathrm{ml}$ PDGF for 2 days). EVs were captured with anti-CD63-coulped beads, detected with a fluorescently labelled anti-CD81 antibody and quantified using flow cytometry. Statistical significance was tested using t-tests. (D) 5.4 $\mathrm{mM} \mathrm{Ca}^{2+}$ increased EV release in hVSMCs (in $2.5 \%$ of EV free FBS, $48 \mathrm{~h}$ ). Statistical significance was tested using t-test. (E) $\mathrm{H}_{2} \mathrm{O}_{2}$ increased EV release in hVSMCs $(2.5 \% \mathrm{FBS}, 24 \mathrm{~h})$. Statistical significance was tested using ANOVA. (F) Nox inhibitor GKT136901 $(10 \mu \mathrm{M})$ decreased EV release in the presence of $5.4 \mathrm{mM} \mathrm{Ca}^{2+}$ in hVSMCs (2.5\% FBS, 24 h). Statistical significance was tested using t-test. (G) Nox5 overexpression in hVSMCs using a lentiviral vector increased EV release.. Statistical significance was tested using the Mann-Whitney test. (H) CFSElabelled EVs $(15 \mu \mathrm{g} / \mathrm{ml})$ from HASMC66 were added to hVSMCs pre-treated with $\mathrm{H}_{2} \mathrm{O}_{2}$ for $20 \mathrm{~h}$ in $2.5 \%$ FBS. Uptake was quantified after $4 \mathrm{~h}$ by flow cytometry. $\mathrm{H}_{2} \mathrm{O}_{2}$ decreased EV uptake. Statistical significance was tested using ANOVA. All graphs show data from 3 independent experiments.

Therefore, we investigated whether uptake of EVs by VSMCs is influenced by ROS. First, we confirmed that EVs secreted by VSMCs present phosphatidylserine, a phagocytosis 
signal (19) (Supplementary Figure 3E) and that CFSE-labelled EVs were phagocytosed when added to synthetic hVSMCs in EV- free medium. Significantly more uptake occurred at 37 ${ }^{\circ} \mathrm{C}$ than at $4{ }^{\circ} \mathrm{C}$ indicative of an active phagocytic process (Supplementary Figure 3F). Next, we showed that $\mathrm{H}_{2} \mathrm{O}_{2}$ inhibited phagocytosis of EVs (Figure $5 \mathrm{H}$ ). Taken together these results suggest that $\mathrm{Ca}^{2+}$-Nox5- induced ROS lead to increased release and inhibited uptake of EVs, which causes their net accumulation and increased calcification in the extracellular environment.

\section{Nox5 is expressed in synthetic VSMCs in human vascular disease tissues}

To examine the relationship between Nox5 expression and VSMC markers in human vascular disease context, we analysed global transcriptomic data from 127 advanced human atherosclerotic plaques (Table 1). Nox5 mRNA expression showed a positive correlation with contractile markers $\alpha$ SMA, smoothelin (SMTN), and myosin light chain (MYL). There was no correlation with CNN1, SM22 $\alpha$ or Myocardin (MYOCD) and a negative correlation with myosin heavy chain (MYH11), one of the first markers lost in de-differentiating VSMCs (34). Unexpectedly, there was a negative correlation with S100A4 and KLF4, which promote

Table 1. Nox5 expression correlates with SMC markers in atherosclerotic plaques. Pearson correlations were performed to investigate the association between the transcriptomic expression levels of Nox 5 and SMC markers, based on the microarray data from human plaques in the BiKE cohort, $\mathrm{n}=127$.

\begin{tabular}{lccccc} 
Cell & Marker & Pearson r & $95 \%$ CI & P (two-tailed) & $\begin{array}{c}\text { P value } \\
\text { summary }\end{array}$ \\
\hline \multirow{3}{*}{ Smooth muscle } & MYH11 & -0.2691 & -0.4240 to -0.09884 & 0.0023 & $* *$ \\
& CNN1 & -0.0161 & -0.1898 to 0.1586 & 0.8574 & ns \\
& MYOCD & 0.1171 & -0.05898 to 0.2862 & 0.1915 & ns \\
& SM22 $\alpha$ & 0.1255 & -0.05048 to 0.2940 & 0.1613 & ns \\
& aSMA & 0.3943 & 0.2356 to 0.5325 & $<0.0001$ & $* * * *$ \\
& MYL10 & 0.4328 & 0.2796 to 0.5644 & $<0.0001$ & $* * * *$ \\
& SMTN & 0.6218 & 0.5014 to 0.7186 & $<0.0001$ & $* * * *$ \\
Synthetic & MYL1 & 0.6692 & 0.5603 to 0.7554 & $<0.0001$ & $* * * *$ \\
& & & & & $* .0003$ \\
Extracellular & S100A4 & -0.313 & -0.4620 to -0.1467 & 0.0003 \\
vesicles & KLF4 & -0.3701 & -0.5113 to -0.2093 & $<0.0001$ & $* * * *$ \\
& & & & & $*$ \\
& CD63 & -0.1774 & -0.3411 to -0.0033 & 0.046 & $* * * *$ \\
& CD81 & -0.3507 & -0.4947 to -0.1880 & $<0.0001$ & $* * * *$ \\
& TSG101 & -0.3624 & -0.5047 to -0.2008 & $<0.0001$ & $* * * *$ \\
Growth factor & SMPD3 & 0.6594 & 0.5482 to 0.7478 & $<0.0001$ & $* * * *$ \\
\hline
\end{tabular}

synthetic differentiation. We also examined EV-related gene expression and found a negative correlation with EV/multivesicular body markers CD63, CD81 and TSG101, but strong positive correlations with CD9 and enzyme regulating EV release SMPD3. 
Supplementary Table 1. Nox5 expression negatively correlates with endothelium, macrophages including lipid-loaded foam cells and lymphocyte markers in atherosclerotic plaques. Pearson correlations were performed based on transcript expression levels in microarray data from the BiKE cohort, $\mathrm{n}=127$ plaques.

\begin{tabular}{|c|c|c|c|c|c|}
\hline Cell & Marker & Pearson $r$ & $95 \% \mathrm{CI}$ & P (two-tailed) & $\begin{array}{l}\mathrm{P} \text { value } \\
\text { summary }\end{array}$ \\
\hline & VWF & -0.2523 & -0.4091 to -0.08092 & 0.0044 & $* *$ \\
\hline \multicolumn{6}{|l|}{ Endothelium } \\
\hline & CD36 & -0.2357 & -0.3938 to -0.06413 & 0.0076 & $* *$ \\
\hline \multicolumn{6}{|l|}{ Foam cell } \\
\hline & FABP4 & -0.2112 & & 0.0171 & $*$ \\
\hline & & & -0.3718 to -0.03843 & & \\
\hline \multirow[t]{3}{*}{ Macrophage } & CD163 & -0.4038 & -0.5405 to -0.2463 & $<0.0001$ & $* * * *$ \\
\hline & CD80 & -0.3709 & -0.5120 to -0.2102 & $<0.0001$ & $* * * *$ \\
\hline & CD86 & -0.2758 & -0.4300 to -0.1060 & 0.0018 & $* *$ \\
\hline \multirow[t]{4}{*}{ Lymphocyte } & ITGAE & -0.4788 & -0.6027 to -0.3323 & $<0.0001$ & $* * * *$ \\
\hline & CD8B & 0.1755 & 0.0006115 to 0.3400 & 0.0493 & $*$ \\
\hline & CD4 & 0.4592 & 0.3091 to 0.5870 & $<0.0001$ & $* * * *$ \\
\hline & FOXP3 & 0.6833 & 0.5774 to 0.7666 & $<0.0001$ & $* * * *$ \\
\hline \multirow[t]{4}{*}{ Other } & TGFB1 & 0.535 & 0.3972 to 0.6491 & $<0.0001$ & $* * * *$ \\
\hline & IFNG & 0.2312 & 0.05865 to 0.3903 & 0.0092 & $* *$ \\
\hline & IL10 & -0.3103 & -0.4603 to -0.1432 & 0.0004 & $* * *$ \\
\hline & HSP90 & -0.5126 & -0.6305 to -0.3716 & $<0.0001$ & $* * * *$ \\
\hline
\end{tabular}

Additionally, there was a positive correlation with PDGF expression. Since atherosclerotic plaques are comprised of many other cell types, we also examined Nox5 expression in correlation with markers of other cell types (Supplementary Table 1). Nox5 expression negatively correlated with endothelial cell, platelet, lipid-laden foam cell, macrophage and some lymphocyte markers (CD8B, CD4, FOXP3). Positive correlation was observed with other lymphocyte markers (ITGAE, CD80, CD86).

To further assess whether Nox5-dependent phenotype switching occurred in human vasculature we localised synthetic and contractile markers and Nox 5 in human arteries on protein level (Figure 6). Immunohistochemical staining of human coronary artery samples showed extensive Nox5 staining in regions with aSMA expression, indicating the presence of VSMCs, but with low expression of contractile marker CNN1 and high expression of synthetic marker S100A4. Some Nox5 staining was present in the adventitia, suggesting possible expression of Nox 5 in other cell types. Taken together, these results support the notion that Nox5 is expressed in synthetic VSMCs in human vessels and that EV release is involved.

\section{Discussion}


In the present study we identify Nox 5 as a key regulator of VSMC phenotype and calcification. We demonstrate that contractile VSMCs have low calcification capacity and that switching towards a synthetic phenotype promotes calcification. We show that extracellular factors known to induce phenotype switching cause increased Nox5 expression. Subsequently, increased Nox 5 expression then induces phenotypic switching to synthetic. We elucidate the mechanism by which synthetic VSMCs are prone to calcification, showing that extracellular $\mathrm{Ca}^{2+}$ enters cells via EVs, which results in a cytosolic $\mathrm{Ca}^{2+}$ rise. This activates Nox5, which is responsible for increased ROS production. Increased ROS lead to decreased contractile marker expression, enhanced EV release and decreased uptake, which promotes calcification (Figure 7).

\section{Contractile VSMCs are resistant to calcification}

In the present study, we demonstrate that contractile VSMCs are resistant to calcification in vitro suggesting that switching towards a synthetic phenotype is required for mineralization. We propose that this is in part due to differences in Nox 5 expression. We show that Nox 5 mRNA expression in advanced atherosclerotic plaques correlated with expression of some SMC markers, and not of markers of other cell types. A negative
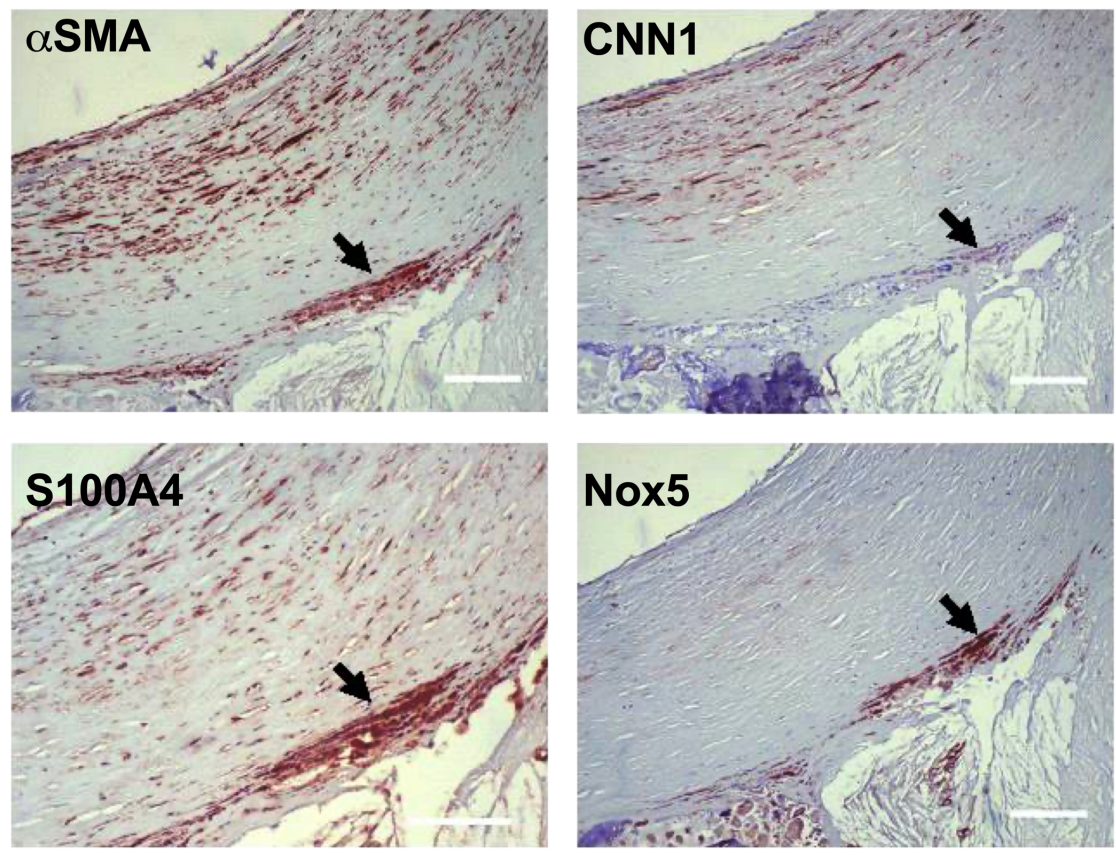

Figure 6. Nox5 is expressed in synthetic cells in human arteries. Immunohistochemical staining of human coronary artery with atherosclerotic plaque. VSMCs were $\alpha$ SMA-positive. Nox 5 was present in VSMCs expressing S100A4, but not contractile marker CNN1 (black arrows). Figure shows representative images from a total of 20 coronary artery and aortic samples that were stained. Scale bars are $250 \mu \mathrm{m}$. 


\section{Chapter 6}

correlation or no correlation was observed with CNN1, SM22 $\alpha$, MYOCD and MYH11, suggesting that Nox5 is expressed in de-differentiated VSMCs. In this dataset we also observed a negative correlation of Nox 5 expression with S100A4 and KLF4, previously associated with synthetic differentiation $(14,30)$. However, the atherosclerotic plaques in this study were of late stages (AHA grade VI and VII). Therefore, it is possible that no active synthetic differentiation occurs at these stages and the correlation is lost even though it may have been present during earlier stages of atherogenesis. Additionally, the negative correlation of S100A4 with Nox 5 expression is in line with our results showing that overexpression of Nox5 or $\mathrm{H}_{2} \mathrm{O}_{2}$-induced oxidative stress downregulated expression of S100A4 protein in VSMCs. S100A4 is known to regulate cell proliferation and motility, but more recently it has been shown to be released to the extracellular space where it has an important function in VSMC phenotype switching (35). Neither of the methods which we employed (mRNA analysis in plaque samples or western blot of cell lysates) would have measured extracellular S100A4 protein accumulation, so its regulation by Nox 5 and ROS cannot be fully excluded.

By immunohistochemistry, we've shown that Nox5 expression in synthetic VSMCs in the vessel wall, consistent with its role as a regulator of phenotypic switching. In line with this, Nox 5 expression was previously shown to be highly upregulated in VSMCs in advanced human atherosclerotic plaques (36), which are characterised by VSMC phenotypic switching and calcification. Additionally, polymorphisms in the Nox5 gene are linked to changes in

A

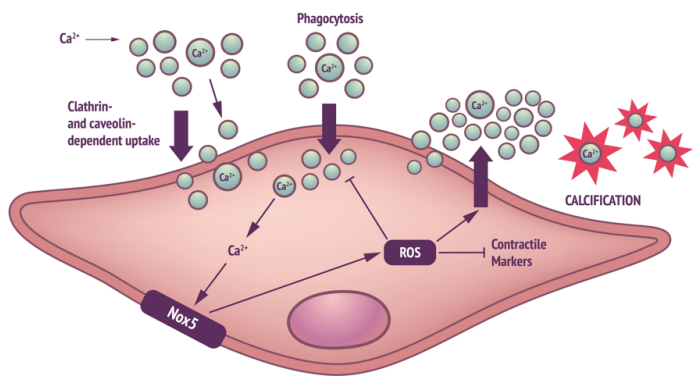

B

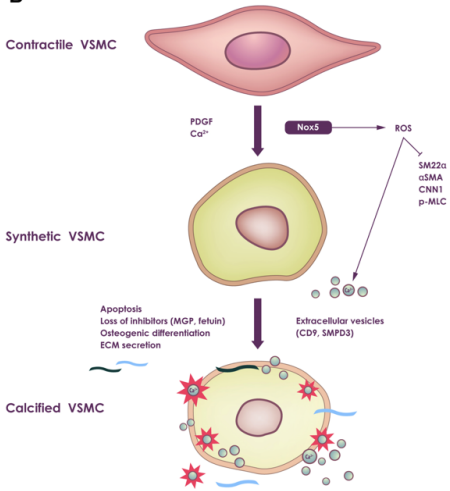

Figure 7. Regulation of VSMC calcification by Nox5. (A) The synthetic phenotype is associated with increased Nox5 expression, which further exacerbates phenotypic switching to synthetic. Extracellular $\mathrm{Ca}^{2+}$ enters cells via clathrin- and caveolin-dependent uptake of EVs, which results in a cytosolic $\mathrm{Ca}^{2+}$ rise. This activates Nox5, which is responsible for increased ROS production. Increased ROS lead to enhanced EV release and decreased phagocytosis. An increased amount of EVs in the extracellular matrix promotes calcification. (B) Contractile VSMCs dedifferentiate to synthetic VSMCs. This process is induced by PDGF, high $\mathrm{Ca}^{2+}$ and mediated by Nox 5 . Synthetic VSMCs undergo further changes, including apoptosis and osteogenic differentiation, secrete increased amounts of ECM (extracellular matrix) and EVs. All these processes lead to increased calcification. 
blood pressure (37), which are associated with VSMC-mediated vascular remodelling. It remains to be established what causes the increase in Nox 5 expression in synthetic VSMCs in the first place. Inflammation-related stimuli have been shown to regulate Nox 5 expression (38), however this has not been investigated in the context of phenotypic switching.

We also found that contractile VSMCs had significantly higher levels of MGP, an inhibitor of vascular calcification. Carboxylated MGP is known to block both crystal formation and BMP signalling (39). An increased level of this inhibitor might offer another explanation to the observed resistance of contractile VSMCs to calcification.

\section{Regulation of VSMC phenotype by Nox5}

We show here that Nox5 is a marker and (via increased ROS production) an inducer of the synthetic phenotype. Consistent with our findings, Nox5-derived ROS were previously identified as a driving force for coronary SMC migration, consistent with the synthetic phenotype, via upregulation of intermediate-conductance $\mathrm{Ca}^{2+}$ activated $\mathrm{K}^{+}$channels (KCNN4) (40). Additionally, Nox5-produced ROS have been shown to mediate PDGFinduced proliferation of VSMCs (41). On the other hand, in other contexts ROS were shown to be required for VSMC contractile differentiation (42) and maintenance of the contractile phenotype via Nox4-derived ROS $(43,44)$. ROS are also required for VSMC contraction (45). It is possible that the discrepancy between our results and previous studies is due to different Nox enzymes mediating divergent effects. The overall outcome for the cells would then depend on the balance between the activity of these enzymes. Another possibility is that ROS form a negative feedback loop for phenotype control, with synthetic cells producing more ROS, which among other effects, help the cells regain a contractile phenotype. This is in line with research showing that Nox 5 is inactivated by oxidation of its $\mathrm{Ca}^{2+}$-binding domain (46). These control mechanisms possibly become dysregulated in vascular pathologies when many adverse factors are at play, as oxidative stress is a causative factor in vascular disease (47).

Our results with regards to Nox5-produced ROS promoting a synthetic phenotype in VSMCs are contrary to recent findings by Montezano et al (48). In this study, human Nox5 was constitutively expressed under the SM22 $\alpha$ promoter in mice, as Nox 5 is absent in the murine genome. The authors observed systemically increased oxidative stress in the transgenic mice, however this was accompanied by increased mesenteric artery contractility and increased expression of contractile markers p-MLC and p-MYPT1. One possible explanation for this contradiction with our results is that in this model Nox 5 expression occurred also during development. The continued presence of Nox 5 since early development might render different effects than increased expression occurring as a result of vascular disease and remodelling in the adult. Nevertheless, this study confirms the detrimental effects of increased ROS in the vasculature (49), since the authors postulate that the uncovered mechanisms are likely relevant to the pathology of hypertension. Additionally, these results 
are in line with our conclusion that Nox 5 is a regulator of VSMC phenotype, but the exact effects of increased Nox 5 are possibly dependent on the physiological context.

\section{Nox5 is the connection between $\mathrm{Ca}^{2+}$ and oxidative stress}

It has been shown before that treating cells with high $\mathrm{Ca}^{2+}$ leads to changes in $\mathrm{Ca}^{2+}$ that result in increased calcification (22). In this study we propose uptake via EVs as a new mechanism of extracellular $\mathrm{Ca}^{2+}$ influencing cytosolic $\mathrm{Ca}^{2+}$ levels. It has been suggested before that high $\mathrm{Ca}^{2+}$ can cause increased loading of $\mathrm{Ca}^{2+}$ into extracellular vesicles (22). As this effect was blocked by the cytosolic $\mathrm{Ca}^{2+}$ chelator BAPTA, $\mathrm{Ca}^{2+}$ uptake into cells was suggested to happen prior to loading into EVs. However, we demonstrate that $\mathrm{Ca}^{2+}$ uptake into EVs can happen in the absence of cells. We also provide evidence that $\mathrm{Ca}^{2+}$-loaded EVs increase cytosolic $\mathrm{Ca}^{2+}$ and that $\mathrm{Ca}^{2+}$ uptake is mediated by clathrin- and caveolin-mediated EV uptake. Taken together, this suggests that two mechanisms of $\mathrm{Ca}^{2+}$ uptake exist - EVdependent and EV-independent, via $\mathrm{Ca}^{2+}$ channels (6). Interestingly, a recent study, which focused on EVs from high-phosphate treated VSMCs demonstrated that these EVs induced an increase in cytosolic $\mathrm{Ca}^{2+}$ partially via release from cytosolic stores (50). Furthermore, it was reported that exosomes bound to autotaxin induced the release of $\mathrm{Ca}^{2+}$ from cytosolic stores (51). We did not investigate the mechanism of $\mathrm{Ca}^{2+}$ elevations in our model. However, it is tempting to speculate that these rises in cytosolic $\mathrm{Ca}^{2+}$ lead to increased Nox 5 activity.

Interestingly, ROS have been shown to increase intracellular $\mathrm{Ca}^{2+}$ by stimulating IP3mediated $\mathrm{Ca}^{2+}$ mobilization, SERCA inhibition, and by stimulating $\mathrm{Ca}^{2+}$ channels (45). This has interesting implications for Nox5 activation and suggests the presence of a positive feedback loop between $\mathrm{Ca}^{2+}$, Nox5 and ROS.

$\mathrm{Ca}^{2+}$ uptake into the arterial vessel wall has been shown to be involved in the pathogenesis of arteriosclerotic lesions in vivo (52) and imbalanced $\mathrm{Ca}^{2+}$ metabolism is known to contribute to medial calcification (6). Here we provide a new mechanism by which $\mathrm{Ca}^{2+}$ induces VSMC calcification, showing that it plays a role in Nox5-dependent ROS generation. Thus, we demonstrate that increased $\mathrm{Ca}^{2+}$ can induce oxidative stress. These findings have implications for vascular disease, as a mineral imbalance in circulation is known to be a manifestation of chronic kidney disease, which is accompanied by vascular calcification (11). Additionally, hitherto advanced glycation end products and oxidized lowdensity lipoproteins were shown to be the main inducers of oxidative stress in the vasculature (31). Although mineral imbalance in chronic kidney disease is known to be associated with ROS accumulation (53), we demonstrate here that in synthetic VSMCs it is $\mathrm{Ca}^{2+}$ itself that contributes to this.

\section{Nox5-mediated oxidative stress induces $E V$ release and inhibits their re-uptake}

EVs are a well-established factor contributing to vascular calcification $(25,54,55)$. Our data indicate that EVs alone, without cells, are sufficient to induce extracellular matrix calcification, demonstrating how potent calcification inducers they are. 
We show here that phenotypic switching is associated with changes in EV release. This is consistent with previous studies (27) where contractile VSMCs showed reduced and synthetic VSMCs increased EV release. However, our study demonstrates for the first time that Nox5 is the molecular link between $\mathrm{Ca}^{2+}$, oxidative stress and EV release. ROS have been shown to promote EV release from other cell types $(56,57)$, however this has not been reported in VSMCs before. Interestingly, $\mathrm{Ca}^{2+}$ can directly stimulate $\mathrm{EV}$ release from cancer cells via $\mathrm{Ca}^{2+}$-dependent Munc13-4 (58). Whether this mechanism is relevant for VSMCs remains to be tested.

It has been shown previously that VSMCs exhibit phagocytic capacity (26) towards phosphatidylserine expressing apoptotic cells and bodies (19) and that EVs present phosphatidylserine (59). Additionally, it has been demonstrated that increased oxidative stress inhibits phagocytosis of apoptotic bodies (60). Our results extend this effect also to secreted EVs and suggest that the increased expression of Nox 5 in concert with increased $\mathrm{Ca}^{2+}$ levels fuels the production of $\mathrm{H}_{2} \mathrm{O}_{2}$ and subsequently limits the phagocytic capacity of VSMCs which together with increased EV release may culminate in increased numbers of EVs in the extracellular matrix.

\section{Limitations and conclusions}

In this study we used both human and porcine VSMCs. While we established that cells of both species react similarly in the experimental conditions in terms of phenotypic switching and calcification, we cannot exclude the possibility of differences. Most importantly, there are species differences in the Nox 5 gene with regards to isoforms $(41,61)$. It remains to be established which human isoforms resemble closest the porcine orthologues.

Another limitation of this study is the use of heparin, which is known to inhibit EV uptake (62), to induce the contractile phenotype in VSMCs. However in our experiments, cells treated with heparin secreted fewer EVs than untreated or synthetic cells. If heparin inhibited EV uptake we would expect to observe an accumulation of EVs and therefore possibly a net increase. That suggests that in our model heparin's EV-blocking activity is weaker than contractile phenotype-promoting activity. However, it cannot be excluded that decreased EV uptake is connected to maintaining contractile differentiation, as these pathways are poorly described in VSMCs.

In conclusion, we showed that switching phenotypes to synthetic is required for VSMC calcification, and that in synthetic cells increased levels of $\mathrm{Ca}^{2+}$-regulated Nox5 play a key role in the generation of oxidative stress and EV release. Inhibition of VSMC phenotypic switching in pathological contexts may be of therapeutic benefit for vascular calcification in vivo and this research identifies Nox 5 as a potential therapeutic target to achieve that.

\section{Acknowledgments}

None.

\section{Sources of Funding}


This study was partly funded by NWO ZonMw (MKMD 40-42600-98-13007) grant to EB, CR and LS. Research from EB, AA, UH, LP, CS, CR and LS, is in part funded via the European Union's Horizon 2020 research and innovation programme under the Marie Sklodowska-Curie grant agreement No 722609. MLBP received funding from the Swiss National Science Foundation (grant \# 310030_166357/1).

\section{Disclosures}

None.

\section{References}

1. Sage AP, Tintut Y, Demer LL. Regulatory mechanisms in vascular calcification. Nat Rev Cardiol. 2010;7(9):528-36.

2. Rennenberg RJ, Kessels AG, Schurgers LJ, van Engelshoven JM, de Leeuw PW, Kroon AA. Vascular calcifications as a marker of increased cardiovascular risk: a meta-analysis. Vasc Health Risk Manag. 2009;5(1):185-97.

3. Lanzer P, Boehm M, Sorribas V, Thiriet M, Janzen J, Zeller T, et al. Medial vascular calcification revisited: review and perspectives. European Heart Journal. 2014;35(23):1515-25.

4. Hoshino T, Chow LA, Hsu JJ, Perlowski AA, Abedin M, Tobis J, et al. Mechanical stress analysis of a rigid inclusion in distensible material: a model of atherosclerotic calcification and plaque vulnerability. American journal of physiology Heart and circulatory physiology. 2009;297(2):H802-10.

5. Farb A, Tang AL, Burke AP, Sessums L, Liang Y, Virmani R. Sudden coronary death. Frequency of active coronary lesions, inactive coronary lesions, and myocardial infarction. Circulation. 1995;92(7):1701-9.

6. Shanahan CM, Crouthamel MH, Kapustin A, Giachelli CM. Arterial Calcification in Chronic Kidney Disease: Key Roles for Calcium and Phosphate. Circulation Research. 2011;109(6):697-711.

7. London GM. Arterial calcification: cardiovascular function and clinical outcome. Nefrologia. 2011;31(6):644-7.

8. Proudfoot D, Skepper JN, Hegyi L, Bennett MR, Shanahan CM, Weissberg PL. Apoptosis Regulates Human Vascular Calcification In Vitro

Evidence for Initiation of Vascular Calcification by Apoptotic Bodies. Circulation Research. 2000;87:105562.

9. Tyson KL, Reynolds, J.L., McNair, R., Zhang, Q., Weissberg, P.L., Shanahan, C.M. Osteo/Chondrocytic Transcription Factors and Their Target Genes Exhibit Distinct Patterns of Expression in Human Arterial Calcification. Arteriosclerosis Thrombosis Vascular Biology. 2003;23(489-494).

10. Kapustin AN, Shanahan CM. Emerging roles for vascular smooth muscle cell exosomes in calcification and coagulation. The Journal of physiology. 2016;594(11):2905-14.

11. Shanahan CM. Mechanisms of vascular calcification in CKD - evidence for premature ageing? Nat Rev Nephrol. 2013;9(11):661-70.

12. Rensen SS, Doevendans PA, van Eys GJ. Regulation and characteristics of vascular smooth muscle cell phenotypic diversity. Netherlands heart journal : monthly journal of the Netherlands Society of Cardiology and the Netherlands Heart Foundation. 2007;15(3):100-8.

13. Alexander MR, Owens GK. Epigenetic Control of Smooth Muscle Cell Differentiation and Phenotypic Switching in Vascular Development and Disease. Annual Review of Physiology. 2012;74(1):13-40.

14. Shankman LS, Gomez D, Cherepanova OA, Salmon M, Alencar GF, Haskins RM, et al. KLF4-dependent phenotypic modulation of smooth muscle cells has a key role in atherosclerotic plaque pathogenesis. Nat Med. 2015;21(6):628-37.

15. Speer MY, Yang H-Y, Brabb T, Leaf E, Look A, Lin W-L, et al. Smooth Muscle Cells Give Rise to Osteochondrogenic Precursors and Chondrocytes in Calcifying Arteries. Circulation Research. 2009;104(6):733-41.

16. Neven E, Persy V, Dauwe S, De Schutter T, De Broe ME, Dhaese PC. Chondrocyte rather than osteoblast conversion of vascular cells underlies medial calcification in uremic rats. Arteriosclerosis, Thrombosis, and Vascular Biology. 2010;30(9):1741-50.

17. Reynolds JE, Joannides, A.J., Skepper, J.N., McNair, R., Schrugers, L.J., Proudfoot, D., Jahnen-Dechent, W., Weissberg, P.L., Shanahan, C.M. Human Vascular Smooth Muscle Cells Undergo Vesicle-Mediated Calcification in Response to Changes in Extracellular Calcium and Phosphate Concentrations: A Potential 
Mechanism for Accelerated Vascular Calcification in ESRD. Journal of the American Society of Nephrology. 2004; 15:2857-67.

18. Hao H, Ropraz P, Verin V, Camenzind E, Geinoz A, Pepper MS, et al. Heterogeneity of Smooth Muscle Cell Populations Cultured From Pig Coronary Artery. Arteriosclerosis, Thrombosis, and Vascular Biology. 2002;22(7):1093-9.

19. Bennett MR, Gibson DF, Schwartz SM, Tait JF. Binding and Phagocytosis of Apoptotic Vascular Smooth Muscle Cells Is Mediated in Part by Exposure of Phosphatidylserine. Circulation Research. 1995;77(6):113642.

20. Proudfoot D, Skepper JN, Hegyi L, Bennett MR, Shanahan CM, Weissberg PL. Apoptosis Regulates Human Vascular Calcification In Vitro : Evidence for Initiation of Vascular Calcification by Apoptotic Bodies. Circ Res. 2000;87(11):1055-62.

21. Dautova Y, Kozlova D, Skepper JN, Epple M, Bootman MD, Proudfoot D. Fetuin-A and Albumin Alter Cytotoxic Effects of Calcium Phosphate Nanoparticles on Human Vascular Smooth Muscle Cells. PLOS ONE. 2014;9(5):e97565.

22. Kapustin AN, Davies JD, Reynolds JL, McNair R, Jones GT, Sidibe A, et al. Calcium regulates key components of vascular smooth muscle cell-derived matrix vesicles to enhance mineralization. Circulation Research. 2011;109(1):e1-e12.

23. Rothwell PM, Pendlebury ST, Wardlaw J, Warlow CP. Critical appraisal of the design and reporting of studies of imaging and measurement of carotid stenosis. Stroke. 2000;31(6):1444-50.

24. Chatrou MLL, Cleutjens JP, van der Vusse GJ, Roijers RB, Mutsaers PHA, Schurgers LJ. Intra-Section Analysis of Human Coronary Arteries Reveals a Potential Role for Micro-Calcifications in Macrophage Recruitment in the Early Stage of Atherosclerosis. PLOS ONE. 2015;10(11):e0142335.

25. Reynolds JL, Joannides AJ, Skepper JN, McNair R, Schurgers LJ, Proudfoot D, et al. Human Vascular Smooth Muscle Cells Undergo Vesicle-Mediated Calcification in Response to Changes in Extracellular Calcium and Phosphate Concentrations: A Potential Mechanism for Accelerated Vascular Calcification in ESRD. J Am Soc Nephrol. 2004;15(11):2857-67.

26. Bennett MR, Evan GI, Schwartz SM. Apoptosis of human vascular smooth muscle cells derived from normal vessels and coronary atherosclerotic plaques. The Journal of Clinical Investigation. 1995;95(5):2266-74.

27. Kapustin AN, Chatrou ML, Drozdov I, Zheng Y, Davidson SM, Soong D, et al. Vascular smooth muscle cell calcification is mediated by regulated exosome secretion. Circ Res. 2015;116(8):1312-23.

28. Chang S, Song S, Lee J, Yoon J, Park J, Choi S, et al. Phenotypic Modulation of Primary Vascular Smooth Muscle Cells by Short-Term Culture on Micropatterned Substrate. PLoS ONE.9(2):e88089.

29. Christen T, Bochaton-Piallat M-L, Neuville P, Rensen S, Redard M, van Eys G, et al. Cultured Porcine Coronary Artery Smooth Muscle Cells A New Model With Advanced Differentiation. Circulation Research. 1999;85(1):99-107.

30. Brisset AC, Hao H, Camenzind E, Bacchetta M, Geinoz A, Sanchez J-C, et al. Intimal Smooth Muscle Cells of Porcine and Human Coronary Artery Express S100A4, a Marker of the Rhomboid Phenotype In Vitro. Circulation Research. 2007;100(7):1055-62.

31. Byon CH, Heath JM, Chen Y. Redox signaling in cardiovascular pathophysiology: A focus on hydrogen peroxide and vascular smooth muscle cells. Redox Biology. 2016;9:244-53.

32. Banfi B, Tirone F, Durussel I, Knisz J, Moskwa P, MolnÃ $i r$ GZn, et al. Mechanism of Ca2+ Activation of the NADPH Oxidase 5 (NOX5). Journal of Biological Chemistry. 2004;279(18):18583-91.

33. Mulcahy LA, Pink RC, Carter DRF. Routes and mechanisms of extracellular vesicle uptake. Journal of Extracellular Vesicles. 2014;3(1):24641.

34. Owens GK, Kumar MS, Wamhoff BR. Molecular Regulation of Vascular Smooth Muscle Cell Differentiation in Development and Disease. Physiological Reviews. 2004;84(3):767-801.

35. Chaabane C, Heizmann CW, Bochaton-Piallat M-L. Extracellular S100A4 induces smooth muscle cell phenotypic transition mediated by RAGE. Biochimica et Biophysica Acta (BBA) - Molecular Cell Research. 2015;1853(9):2144-57.

36. Guzik TJ, Chen W, Gongora MC, Guzik B, Lob HE, Mangalat D, et al. Calcium-Dependent NOX5 Nicotinamide Adenine Dinucleotide Phosphate Oxidase Contributes to Vascular Oxidative Stress in Human Coronary Artery Disease. Journal of the American College of Cardiology. 2008;52(22):1803-9.

37. Kraja AT, Cook JP, Warren HR, Surendran P, Liu C, Evangelou E, et al. New Blood Pressure-Associated Loci Identified in Meta-Analyses of 475000 Individuals. Circulation Cardiovascular genetics. 2017;10(5).

38. Manea A, Manea SA, Florea IC, Luca CM, Raicu M. Positive regulation of NADPH oxidase 5 by proinflammatory-related mechanisms in human aortic smooth muscle cells. Free Radical Biology and Medicine. 2012;52(9):1497-507.

39. Schurgers LJ, Uitto J, Reutelingsperger CP. Vitamin K-dependent carboxylation of matrix Gla-protein: a crucial switch to control ectopic mineralization. Trends in Molecular Medicine. 2013;19(4):217-26. 
40. Gole HKA, Tharp DL, Bowles DK. Upregulation of Intermediate-Conductance Ca2+-Activated K+ Channels (KCNN4) in Porcine Coronary Smooth Muscle Requires NADPH Oxidase 5 (NOX5). PLOS ONE. 2014;9(8):e105337.

41. Jay DB, Papaharalambus CA, Seidel-Rogol B, Dikalova AE, Lassègue B, Griendling KK. Nox5 mediates PDGF-induced proliferation in human aortic smooth muscle cells. Free Radical Biology and Medicine. 2008;45(3):329-35.

42. Su B, Mitra S, Gregg H, Flavahan S, Chotani MA, Clark KR, et al. Redox Regulation of Vascular Smooth Muscle Cell Differentiation. Circulation Research. 2001;89(1):39-46.

43. Clempus RE, Sorescu D, Dikalova AE, Pounkova L, Jo P, Sorescu GP, et al. Nox4 Is Required for Maintenance of the Differentiated Vascular Smooth Muscle Cell Phenotype. Arteriosclerosis, Thrombosis, and Vascular Biology. 2007;27(1):42-8.

44. Xiao Q, Luo Z, Pepe AE, Margariti A, Zeng L, Xu Q. Embryonic stem cell differentiation into smooth muscle cells is mediated by Nox4-produced H2O2. American Journal of Physiology-Cell Physiology. 2009;296(4):C711-C23.

45. Touyz RM, Alves-Lopes R, Rios FJ, Camargo LL, Anagnostopoulou A, Arner A, et al. Vascular smooth muscle contraction in hypertension. Cardiovascular research. 2018;114(4):529-39.

46. Petrushanko IY, Lobachev VM, Kononikhin AS, Makarov AA, Devred F, Kovacic H, et al. Oxidation of $\mathrm{Ca} 2+-$ Binding Domain of NADPH Oxidase 5 (NOX5): Toward Understanding the Mechanism of Inactivation of NOX5 by ROS. PLOS ONE. 2016;11(7):e0158726.

47. Griendling KK, Sorescu D, Ushio-Fukai M. NAD(P)H Oxidase: Role in Cardiovascular Biology and Disease. Circulation Research. 2000;86(5):494-501.

48. Montezano AC, De Lucca Camargo L, Persson P, Rios FJ, Harvey AP, Anagnostopoulou A, et al. NADPH Oxidase 5 Is a Pro-Contractile Nox Isoform and a Point of Cross-Talk for Calcium and Redox SignalingImplications in Vascular Function. Journal of the American Heart Association. 2018;7(12).

49. Münzel T, Camici GG, Maack C, Bonetti NR, Fuster V, Kovacic JC. Impact of Oxidative Stress on the Heart and Vasculature: Part 2 of a 3-Part Series. Journal of the American College of Cardiology. 2017;70(2):212-29.

50. Chen NX, O'Neill KD, Moe SM. Matrix vesicles induce calcification of recipient vascular smooth muscle cells through multiple signaling pathways. Kidney International. 2017.

51. Jethwa SA, Leah EJ, Zhang Q, Bright NA, Oxley D, Bootman MD, et al. Exosomes bind autotaxin and act as a physiological delivery mechanism to stimulate LPA receptor signalling in cells. Journal of Cell Science. 2016.

52. Fleckenstein-Grün G, Frey M, Thimm F, Fleckenstein A. Protective effects of various calcium antagonists against experimental arteriosclerosis. J Hum Hypertens. 1992;6 Suppl 1:S13-8.

53. Scholze A, Jankowski J, Pedraza-Chaverri J, Evenepoel P. Oxidative Stress in Chronic Kidney Disease. Oxidative medicine and cellular longevity. 2016;2016:8375186-.

54. New SE, Goettsch C, Aikawa M, Marchini JF, Shibasaki M, Yabusaki K, et al. Macrophage-Derived Matrix Vesicles: An Alternative Novel Mechanism for Microcalcification in Atherosclerotic Plaques. Circulation Research.

55. Dai XY, Zhao MM, Cai Y, Guan QC, Zhao Y, Guan Y, et al. Phosphate-induced autophagy counteracts vascular calcification by reducing matrix vesicle release. Kidney Int.83(6):1042-51.

56. Carver KA, Yang D. N-Acetylcysteine Amide Protects Against Oxidative Stress-Induced Microparticle Release From Human Retinal Pigment Epithelial Cells. Investigative Ophthalmology \& Visual Science. 2016;57(2):360-71.

57. Cui L, Houston DA, Farquharson C, MacRae VE. Characterisation of matrix vesicles in skeletal and soft tissue mineralisation. Bone.87:147-58.

58. Messenger SW, Woo SS, Sun Z, Martin TFJ. A Ca $<$ sup $>2+</$ sup $>$-stimulated exosome release pathway in cancer cells is regulated by Munc13-4. The Journal of Cell Biology. 2018;217(8):2877-90.

59. Arraud N, Linares R, Tan S, Gounou C, Pasquet JM, Mornet S, et al. Extracellular vesicles from blood plasma: determination of their morphology, size, phenotype and concentration. Journal of Thrombosis and Haemostasis. 2014;12(5):614-27.

60. Anderson HA, Englert R, Gursel I, Shacter E. Oxidative stress inhibits the phagocytosis of apoptotic cells that have externalized phosphatidylserine. Cell death and differentiation. 2002;9(6):616-25.

61. Bedard K, Jaquet V, Krause K-H. NOX5: from basic biology to signaling and disease. Free Radical Biology and Medicine. 2012;52(4):725-34.

62. Atai NA, Balaj L, van Veen H, Breakefield XO, Jarzyna PA, Van Noorden CJF, et al. Heparin blocks transfer of extracellular vesicles between donor and recipient cells. Journal of Neuro-Oncology. 2013;115(3):343-51. 
NOX5 A NOVEL KEY FACTOR IN VSMC PHENOTYPE SWITCHING 


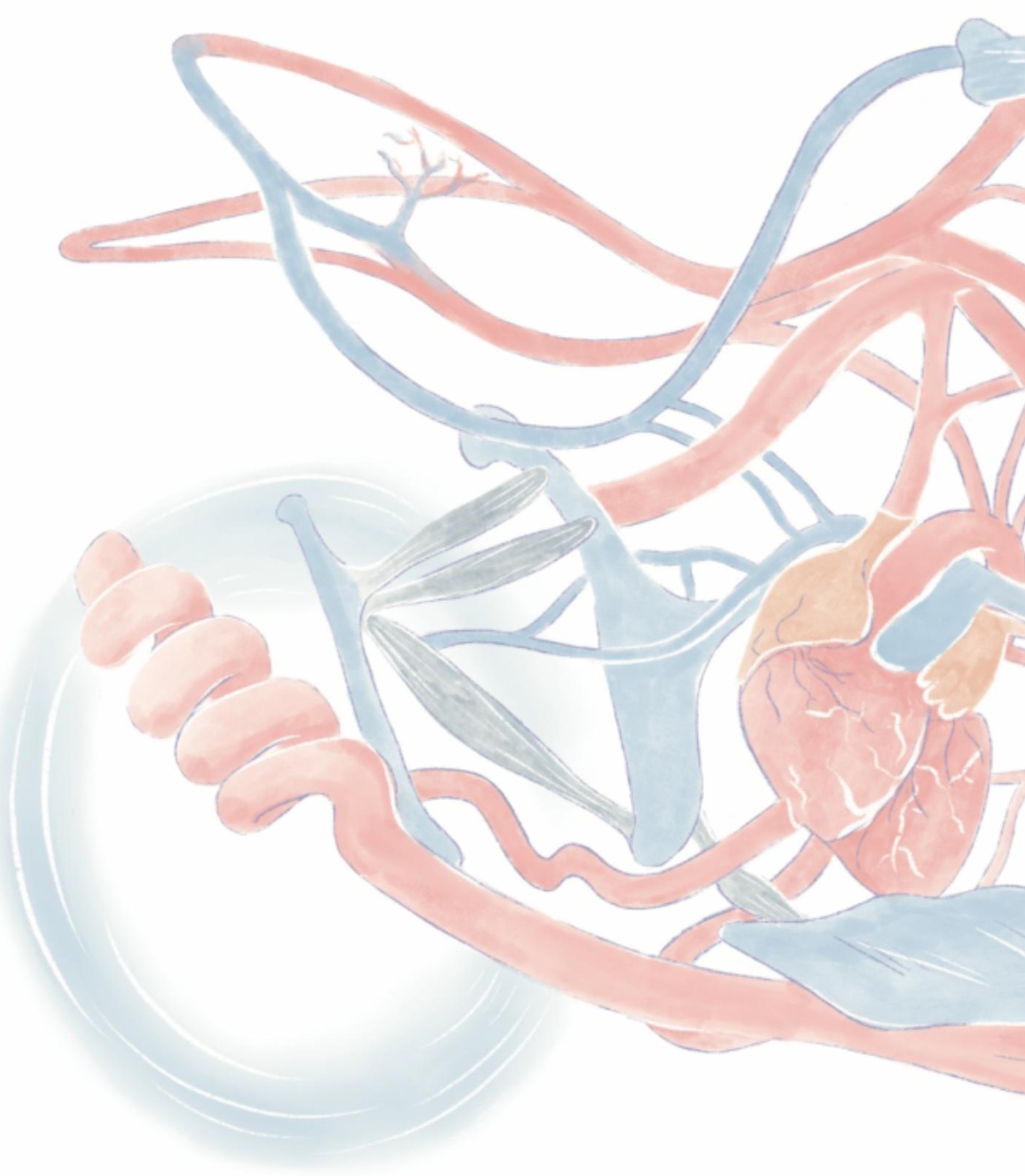




\section{Chapter 7}

General discussion 
Cardiovascular disease (CVD) is one of the leading causes of death worldwide, especially due to myocardial infarction and stroke (WHO 2018). Both myocardial infarction and stroke are the result of atherothrombosis. Atherothrombosis is a consequence of atherosclerotic plaque rupture or erosion, causing local thrombus formation thereby occluding the vessel wall lumen (1). Oral anticoagulants are prescribed to protect patients from (recurrent) thrombosis. For decades, vitamin K antagonists (VKA) are used as longterm oral anticoagulant treatment. However, unfavourable pharmacokinetics of VKA resulted in the development of direct non-vitamin $\mathrm{K}$ antagonist oral anticoagulants (NOAC). NOAC specifically target a single coagulation protein in the coagulation cascade, which is either factor Xa or factor IIa (thrombin). While VKA treatment is shown to detrimentally influence the vasculature by increasing vascular calcification and plaque vulnerability (2), NOACs have shown beneficial effects on the vasculature $(3,4)$. The effects of VKA on the vessel wall are mediated by vitamin K dependent proteins (VKDP), while NOAC inhibit FXa or thrombin mediated protease activated receptor (PAR) signalling (5).

Vascular calcification is associated with increased cardiovascular risk and presence of calcification at any vascular site increases the risk for mortality and cardiovascular events by some 4-fold (6). Consequences of vascular calcification are dependent on volume, density and metabolic activity of the calcification (7-9). Calcification in the medial layer of the vessel wall is associated with hypertension and vascular stiffness, while calcification in the intimal layer has an impact on plaque stability. In patients, progression of coronary artery calcification (CAC) of $25 \%$ annually is associated with an increased vascular mortality risk of some 17-fold (10).

Vascular smooth muscle cells (VSMC) are crucial in maintaining vessel wall integrity, but also pivotal players in controlling and initiation of vascular calcification via phenotype switching, exosome release and local synthesis of the vitamin $\mathrm{K}$ dependent calcification inhibitor matrix Gla protein (MGP) (11). VKDP are activated via gammaglutamylcarboxylation which requires vitamin $\mathrm{K}$ as cofactor. VKA treatment increases vascular calcification by inducing vitamin $\mathrm{K}$ deficiency, subsequently limiting carboxylation and thereby activity of MGP. Next to drug-induced vitamin K deficiency, vitamin K deficiency can be the result of limited dietary uptake or intake of vitamin $\mathrm{K}$ due to intestinal disease or dietary restriction. Vitamin $\mathrm{K}$ supplementation can replenish vitamin $\mathrm{K}$ stores to support carboxylation, thereby providing a possibility to counteract calcification pressure.

The research presented in this thesis focuses on the involvement of VSMC in relation to vascular calcification and development of atherosclerosis. The results described in this thesis will impact on anticoagulation treatment strategy and opens up novel strategies to limit progression of vascular calcification. In chapter 3 we investigated consequences of atherosclerotic plaque calcification on hypercoagulability. In chapter $\mathbf{4}$, we investigated the role of vitamin $\mathrm{K}$ supplementation in a preclinical model of atherosclerosis and underlying vitamin $\mathrm{K}$ metabolism in VSMC. In chapter 5, we compared effects of warfarin and dabigatran on development of atherosclerosis and calcification with a specific focus on the 
underlying role of VSMC. Chapter 6 focused on the role of Nox 5 as novel key regulator of VSMC phenotypic switching and subsequent exosome mediated calcification.

Key findings of this thesis are:

I. Calcified atherosclerotic plaques cause increased hypercoagulability (Chapter 3).

II. Discontinuing short term VKA treatment prevents detrimental future vascular effects (Chapter 4).

III. Vitamin $\mathrm{K}$ holds antioxidant properties and thereby improves atherosclerotic plaque stability (Chapter 4)

IV. Dabigatran treatment has no impact on vascular calcification, yet reduces atherogenesis (Chapter 5).

V. Depth of anticoagulation with VKA treatment significantly correlates with vascular calcification (Chapter 5).

VI. Nox5 is a novel key-factor for VSMC phenotypic switching and inhibition of Nox5 inhibits vascular calcification (Chapter 6).

\section{Calcification of atherosclerotic plaques increases risk on atherothrombosis via hypercoagulability.}

Elevated thrombin generation has a positive association with presence and severity of coronary atherosclerosis and coronary artery calcification (CAC) (12), suggesting an interplay between coagulation and calcification. In this thesis, we show that warfarin promotes and accelerates calcification of atherosclerotic plaques which is accompanied by the induction of a prethrombotic state. A prethrombotic state is associated with hypercoagulability (13), which adversely influences atherogenesis (14). The underlying mechanism by which calcification indirectly promotes coagulation can be attributed to VSMC phenotypic switching. I showed that VSMC switching from a contractile towards a synthetic phenotype contributes to increased endogenous thrombin potential, which is even further elevated when VSMC are calcified. VSMC induced coagulation is mainly mediated via the extrinsic coagulation pathway by increased tissue factor activity. Our findings are in line with previous reports showing that CAC is associated with higher thrombinantithrombin (TAT) complex levels $(12,15)$. Factor IXa-antithrombin (FIXa:AT) complex is a marker reflecting early activation of the coagulation cascade, while TAT reflects the later stage of the coagulation cascade. Elevated levels of these complexes point towards a prethrombotic state and subsequently hypercoagulability (13). Vascular calcification is associated with increased cardiovascular risk $(6,16)$ and our data may explain in part this increased risk by the underlying hypercoagulability due to vascular calcification. Future clinical studies addressing the role of vascular calcification and hypercoagulability on clinical outcome will provide more insight in this process. 


\section{VKA induced vascular calcification increases plaque vulnerability.}

Recently ${ }^{18} \mathrm{~F}-\mathrm{NaF}$ has emerged as imaging modality to visualize active calcification, which is associated with plaque vulnerability $(9,17)$. Active calcification indicates a metabolic active process, which will eventually develop in macrocalcification $(18,19)$. Calcification progression is a strong predictor of increased vascular mortality (10). Also microcalcification, or spotty calcification, is acknowledged to increase plaque vulnerability. Microcalcification in the atherosclerotic fibrous cap increases plaque vulnerability by inducing local stress (20) and is more frequently present in culprit lesions of patients with acute myocardial infarction or unstable angina (21). Moreover, presence of microcalcification is accompanied by increased atheroma volume at baseline and atheroma progression (22). In this thesis, we measured active calcification using ${ }^{18} \mathrm{~F}-\mathrm{NaF}$ and demonstrated increased vascular ${ }^{18} \mathrm{~F}-\mathrm{NaF}$ uptake after warfarin treatment. Analysing atherosclerotic plaque environment using (immuno)histochemistry showed that warfarin increased markers of plaque vulnerability. These data are in line with previous reports in mice and man showing detrimental effect of VKA by promoting vascular calcification and inducing an unstable plaque phenotype $(2,23)$.

In the clinic, vascular calcification is usually visualized using computed tomography (CT). The current resolution of CT limits detection of calcification to sheet like calcification, known as macrocalcification (24). Nevertheless, coronary CT is used to calculate the Agatston score, which indicates presence of vascular calcification. The Agatston score is based on both volume and density of calcification in the coronary arteries. Recently, vascular calcification volume and calcification density were investigated separately (8). It was shown that calcification density improves plaque stability, whereas increased calcification volume negatively impacts plaque vulnerability. We showed that VKA treatment increased calcification area in the atherosclerotic plaque. Similar to VKA, statins have been shown to increase vascular calcification. However, while both increase vascular calcification statins increases predominantly calcification density (25). This indicates that different therapeutic interventions may result in different vascular calcification effects and thus different cardiovascular risk outcome (26). Accordingly, no clear association is present between CAC and atherothrombotic risk (27). Future clinical studies should address this by comparing warfarin and statin use in relation to vascular calcification and cardiovascular outcome.

\section{Vitamin $K$ supplementation beneficially affects atherosclerotic plaque development.}

Vascular calcification is inhibited by calcification inhibitors such as MGP. The vascular function of MGP was revealed by experiments in mice lacking MGP resulting in early death due to severe vascular calcification. MGP has been shown to accumulate at areas of calcification $(28,29)$. The upregulation of MGP suggests that it acts in response to vascular calcification, however the predominant form of MGP around calcification areas is inactive 
ucMGP. The increased synthesis of MGP requires vitamin $\mathrm{K}$ as cofactor for carboxylation, and thus increased ucMGP levels point towards local deficiency or defect of vitamin $\mathrm{K}$ metabolism. VKA treatment limits vitamin $\mathrm{K}$ recycling and thus induces vitamin $\mathrm{K}$ deficiency, subsequently elevating ucMGP (11). Here, we investigated whether vitamin K supplementation after initial VKA treatment can hold or reduce calcification and atherosclerosis. Our data show that discontinuing VKA limits vascular calcification progression and supplementation of MK7 reduces atherosclerotic plaque size. Moreover, using a novel imaging probe, exogenous labelled fetuin-A, presence of calcification nidus was reduced in atherosclerotic plaques of MK7 treated animals. Fetuin-A is a systemic calcification inhibitor that is internalized and secreted by vesicles of VSMC $(30,31)$. FetuinA and MGP are found in extracellular vesicles to control calcification. Presence of fetuin-A in extracellular vesicles promotes vesicles phagocytosis and blocks vesicle-mediated VSMC calcification (31), thus limiting active calcification. I showed that MK7 reduced secretion of VSMC extracellular vesicles. Thus, reduced presence of fetuin-A after MK7 treatment suggest reduced release of extracellular vesicles, thereby potentially limiting active calcification.

Oxidative stress is known to play a crucial role in cardiovascular disease. Additionally, oxidative stress is an inducer of vascular calcification. The potential of vitamin $\mathrm{K}$ to reduce vascular oxidative stress has recently been put forward (32). We showed that MK7 reduced VSMC oxidative stress both in vivo and in vitro. The contribution of oxidative stress in cardiovascular disease is highlighted in chapter 6, where we showed that Nox 5 is a novel inducer of VSMC oxidative stress and calcification. While the Nox family consists of 5 subtypes, only Nox5 is activated in a calcium-dependent manner (33). Nox5 is significantly increased under inflammatory conditions and in human atherosclerotic plaques (34). We showed that Nox5 regulates VSMC phenotypical switching. Contractile VSMC are key in regulating vascular tone and integrity (35), but are also resistant to calcification. In contrast, synthetic VSMC are prone to enhance oxidative stress and calcification. Nox5 drives oxidative stress resulting in synthetic VSMC differentiation, subsequently elevating extracellular vesicle secretion and calcification. We showed that silencing or blocking Nox5 prevents oxidative stress and inhibits VSMC mediated calcification. Taken together, the identification of Nox 5 as a novel pathological marker and the beneficial effects of vitamin $\mathrm{K}$ paves the way for novel therapeutic approach.

\section{The NOAC Dabigatran Etexilate does not induce vascular calcification}

With the clinical approval of NOACs, an alternative for VKAs as long-term oral anticoagulant treatment is now available (36-39). While VKA treatment has been shown to aggravate atherosclerosis (this thesis, (2)), initial studies with NOACs showed beneficial effects on atherogenesis. Both thrombin and factor $\mathrm{Xa}$ inhibitors have shown to reduce plaque size and improving plaque vulnerability $(3,4,40,41)$. 
However, a direct comparison between VKA and NOAC was lacking. We show that dabigatran does not induce vascular calcification. This is in line with unpublished results finding reduced intimal calcification with rivaroxaban (4). In this thesis we show aggravating effects of warfarin on calcification and atherogenesis, while dabigatran lacks an effect on calcification and shows beneficial effects on atherogenesis. Similarly, clinical studies assessing vascular calcification show increased vascular calcification with VKA treatment which is absent after NOAC treatment (42). Moreover, VKA treatment increases atherosclerotic plaque burden and high-risk plaque features, while NOAC lowered high-risk plaque features (23).

The beneficial effects of NOACs are likely via inhibition of thrombin and factor Xa mediated protease activated receptor (PAR) signalling. Thrombin activates PAR1, 3 and 4, and dabigatran has been shown to block thrombin induced PAR1 signalling (43). Similar to dabigatran treatment, deletion of PAR1 in $A p o e^{-/-}$mice attenuates atherosclerosis development (44). Interestingly, direct thrombin inhibition with bivalirudin (synthetic form of the natural thrombin inhibitor hirudin) had no benefit on atherosclerotic plaques development (45), suggesting different effects on PAR signalling. Furthermore, PAR1 activation is not limited to thrombin, and PAR1 can be cleaved by other proteases to induce cytoprotective signalling (46). PAR1 antagonist and MMP1 inhibition showed reduction of atherosclerosis progression (45). Mutations in PAR cleavage sites specific for thrombin or FXa could provide important insight in the protective mechanisms of NOACs on the vasculature.

\section{Concluding remarks and future perspectives}

The aim of my thesis was to gain further insight in the effects of (anti)coagulation on development of atherosclerosis and calcification, focussing on the contribution of VSMC (summarized in Figure 1). I provide evidence that there is an interplay between calcification and coagulation, in that vascular calcification causes a prethrombotic state. Additionally, I provide an explanation why the extent of vascular calcification is linked to cardiovascular mortality by providing a strong catalytic surface when exposed to the bloodstream likely causing lethal atherothrombosis. Moreover, I show that phenotypic switching of VSMC increases the coagulation potential and supports initiation of calcification. Next, we identified Nox5 as a novel regulator of oxidative stress induced VSMC phenotypic switching. Subsequently, we unravelled a novel mechanism by which oxidative stress and extracellular vesicle release promotes calcification. In addition, I provide data that vitamin $\mathrm{K}$ can reduce oxidative stress, extracellular vesicle release and subsequently limit active calcification. Finally, our work stresses the importance of a personalised medicine approach in which patients with pre-existing atherosclerosis would benefit from NOAC treatment. 


\section{Vitamin K Antagonist}

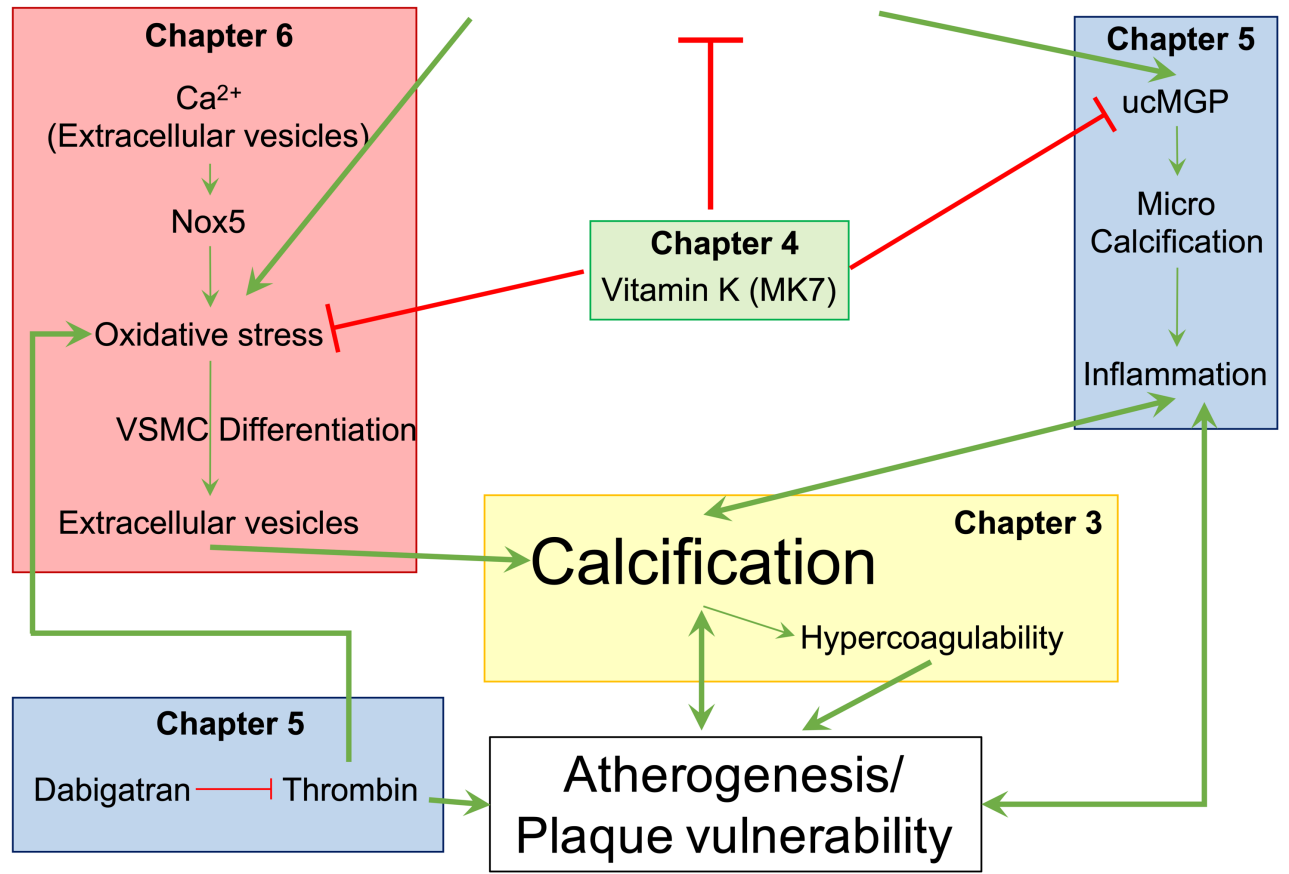

Figure 1. Overview of research findings in this thesis. The interplay between calcification and coagulation was evaluated in chapter 3. Here, calcification promoted hypercoagulability, which subsequently could promote atherogenesis. In chapter 4, we show that MK7, a subform of vitamin K, reduces oxidative stress and blocks warfarin mediated effects on VSMC. Comparison of warfarin and dabigatran on atherogenesis in chapter 5 showed aggravating effects of VKA by promoting inflammation and active calcification, while dabigatran reduced atherogenesis. Finally, we reveal Nox5 as a novel key regulator of VSMC phenotype switching via oxidative stress in chapter 6.

\section{References}

1. Libby P, Ridker PM. Inflammation and Atherothrombosis. J Am Coll Cardiol. 2006 Nov;48(9):A33-A46.

2. Schurgers LJ, Joosen IA, Laufer EM, Chatrou MLL, Herfs M, Winkens MHM, et al. Vitamin Kantagonists accelerate atherosclerotic calcification and induce a vulnerable plaque phenotype. PLoS ONE. 2012;7(8):e43229.

3. Kadoglou NPE, Moustardas P, Katsimpoulas M, Kapelouzou A, Kostomitsopoulos N, Schafer K, et al. The beneficial effects of a direct thrombin inhibitor, dabigatran etexilate, on the development and stability of atherosclerotic lesions in apolipoprotein E-deficient mice : dabigatran etexilate and atherosclerosis. Cardiovasc Drugs Ther. 2012 Oct;26(5):367-74.

4. Posthuma JJ, Posma JJN, van Oerle R, Leenders P, van Gorp RH, Jaminon AMG, et al. Targeting Coagulation Factor Xa Promotes Regression of Advanced Atherosclerosis in Apolipoprotein-E Deficient Mice. Sci Rep. Nature Publishing Group; 2019 Mar 7;9(1):3909.

5. van Gorp RH, Schurgers LJ. New Insights into the Pros and Cons of the Clinical Use of Vitamin K Antagonists (VKAs) Versus Direct Oral Anticoagulants (DOACs). Nutrients. 2015;7(11):9538-57.

6. Rennenberg RJMW, Kessels AGH, Schurgers LJ, van Engelshoven JMA, de Leeuw PW, Kroon AA. Vascular calcifications as a marker of increased cardiovascular risk: a meta-analysis. Vasc Health Risk Manag. 2009;5(1):185-97. 
7. Kelly-Arnold A, Maldonado N, Laudier D, Aikawa E, Cardoso L, Weinbaum S. Revised microcalcification hypothesis for fibrous cap rupture in human coronary arteries. Proc Natl Acad Sci USA. National Academy of Sciences; 2013 Jun 25;110(26):10741-6.

8. Criqui MH, Knox JB, Denenberg JO, Forbang NI, McClelland RL, Novotny TE, et al. Coronary Artery Calcium Volume and Density: Potential Interactions and Overall Predictive Value: The Multi-Ethnic Study of Atherosclerosis. JACC Cardiovasc Imaging. 2017 Aug;10(8):845-54.

9. Joshi NV, Vesey AT, Williams MC, Shah ASV, Calvert PA, Craighead FHM, et al. 18F-fluoride positron emission tomography for identification of ruptured and high-risk coronary atherosclerotic plaques: a prospective clinical trial. Lancet. 2014 Feb 22;383(9918):705-13.

10. Raggi P, Callister TQ, Shaw LJ. Progression of coronary artery calcium and risk of first myocardial infarction in patients receiving cholesterol-lowering therapy. Arterioscler Thromb Vasc Biol. American Heart Association, Inc; $2004 \mathrm{Jul} ; 24(7): 1272-7$.

11. Schurgers LJ, Cranenburg ECM, Vermeer C. Matrix Gla-protein: the calcification inhibitor in need of vitamin K. Thromb Haemost. 2008 Oct;100(4):593-603.

12. Borissoff JI, Joosen IA, Versteylen MO, Spronk HM, Cate ten H, Hofstra L. Accelerated in vivo thrombin formation independently predicts the presence and severity of CT angiographic coronary atherosclerosis. JACC Cardiovasc Imaging. 2012 Dec;5(12):1201-10.

13. Hobbelt AH, Spronk HM, Crijns HJGM, Cate ten H, Rienstra M, Van Gelder IC. Prethrombotic State in Young Very Low-Risk Patients With Atrial Fibrillation. J Am Coll Cardiol. 2017 Apr 18;69(15):1990-2.

14. Borissoff JI, Heeneman S, Kilinç E, Kassák P, van Oerle R, Winckers K, et al. Early atherosclerosis exhibits an enhanced procoagulant state. Circulation. $2010 \mathrm{Aug} 24 ; 122(8): 821-30$.

15. Horn P, Erkilet G, Veulemans V, Kröpil P, Schurgers L, Zeus T, et al. Microparticle-Induced Coagulation Relates to Coronary Artery Atherosclerosis in Severe Aortic Valve Stenosis. PLoS ONE. Public Library of Science; 2016;11(3):e0151499.

16. Budoff MJ, Young R, Burke G, Jeffrey Carr J, Detrano RC, Folsom AR, et al. Ten-year association of coronary artery calcium with atherosclerotic cardiovascular disease (ASCVD) events: the multi-ethnic study of atherosclerosis (MESA). Eur Heart J. 2018 Jul 1;39(25):2401-8.

17. Wang Y, Osborne MT, Tung B, Li M, Li Y. Imaging Cardiovascular Calcification. J Am Heart Assoc. 2018 Jun 28;7(13):233.

18. Pawade TA, Newby DE, Dweck MR. Calcification in Aortic Stenosis. J Am Coll Cardiol. 2015 Aug;66(5):561-77.

19. Dweck MR, Jenkins WSA, Vesey AT, Pringle MAH, Chin CWL, Malley TS, et al. 18F-sodium fluoride uptake is a marker of active calcification and disease progression in patients with aortic stenosis. Circ Cardiovasc Imaging. 2014 Mar;7(2):371-8.

20. Vengrenyuk Y, Carlier S, Xanthos S, Cardoso L, Ganatos P, Virmani R, et al. A hypothesis for vulnerable plaque rupture due to stress-induced debonding around cellular microcalcifications in thin fibrous caps. Proc Natl Acad Sci USA. 2006 Oct 3;103(40):14678-83.

21. Ehara S, Kobayashi Y, Yoshiyama M, Shimada K, Shimada Y, Fukuda D, et al. Spotty calcification typifies the culprit plaque in patients with acute myocardial infarction: an intravascular ultrasound study. Circulation. 2004 Nov 30;110(22):3424-9.

22. Kataoka Y, Wolski K, Uno K, Puri R, Tuzcu EM, Nissen SE, et al. Spotty calcification as a marker of accelerated progression of coronary atherosclerosis: insights from serial intravascular ultrasound. J Am Coll Cardiol. 2012 May 1;59(18):1592-7.

23. Plank F, Beyer C, Friedrich G, Stühlinger M, Hintringer F, Dichtl W, et al. Influence of vitamin K antagonists and direct oral anticoagulation on coronary artery disease: A CTA analysis. International Journal of Cardiology. Elsevier B.V; 2018 Jun 1;260(C):11-5.

24. Budoff MJ, Dowe D, Jollis JG, Gitter M, Sutherland J, Halamert E, et al. Diagnostic performance of 64multidetector row coronary computed tomographic angiography for evaluation of coronary artery stenosis in individuals without known coronary artery disease: results from the prospective multicenter ACCURACY (Assessment by Coronary Computed Tomographic Angiography of Individuals Undergoing Invasive Coronary Angiography) trial. J Am Coll Cardiol. 2008 Nov 18;52(21):1724-32.

25. Kovarnik T, Mintz GS, Skalicka H, Kral A, Horak J, Skulec R, et al. Virtual histology evaluation of atherosclerosis regression during atorvastatin and ezetimibe administration: HEAVEN study. Circ J. The Japanese Circulation Society; 2012;76(1):176-83.

26. Reutelingsperger C, Schurgers L. Coronary Artery Calcification: A Janus-Faced Biomarker? JACC Cardiovasc Imaging. 2018 Sep;11(9):1324-6.

27. Rodriguez-Granillo GA, Carrascosa P, Bruining N. Progression of coronary artery calcification at the crossroads: sign of progression or stabilization of coronary atherosclerosis? Cardiovasc Diagn Ther. 2016 Jun;6(3):250-8. 
28. Schurgers LJ, Teunissen KJF, Knapen MHJ, Kwaijtaal M, van Diest R, Appels A, et al. Novel conformation-specific antibodies against matrix gamma-carboxyglutamic acid (Gla) protein: undercarboxylated matrix Gla protein as marker for vascular calcification. Arterioscler Thromb Vasc Biol. 2005 Aug;25(8):1629-33.

29. Proudfoot D, Shanahan CM. Molecular mechanisms mediating vascular calcification: role of matrix Gla protein. Nephrology (Carlton). John Wiley \& Sons, Ltd (10.1111); 2006 Oct;11(5):455-61.

30. Kapustin AN, Chatrou MLL, Drozdov I, Zheng Y, Davidson SM, Soong D, et al. Vascular smooth muscle cell calcification is mediated by regulated exosome secretion. Circ Res. Lippincott Williams \& Wilkins; 2015 Apr 10;116(8):1312-23.

31. Reynolds JL, Skepper JN, McNair R, Kasama T, Gupta K, Weissberg PL, et al. Multifunctional roles for serum protein fetuin-a in inhibition of human vascular smooth muscle cell calcification. Journal of the American Society of Nephrology. American Society of Nephrology; 2005 Oct;16(10):2920-30.

32. Petsophonsakul P, Furmanik M, Forsythe R, Dweck M, Schurink GW, Natour E, et al. Role of Vascular Smooth Muscle Cell Phenotypic Switching and Calcification in Aortic Aneurysm Formation. Arterioscler Thromb Vasc Biol. Lippincott Williams \& Wilkins Hagerstown, MD; 2019 Jul;39(7):1351-68.

33. Touyz RM, Anagnostopoulou A, Rios F, Montezano AC, Camargo LL. NOX5: Molecular biology and pathophysiology. Exp Physiol. John Wiley \& Sons, Ltd (10.1111); 2019 May;104(5):605-16.

34. Vlad M-L, Manea S-A, Lazar A-G, Raicu M, Muresian H, Simionescu M, et al. Histone AcetyltransferaseDependent Pathways Mediate Upregulation of NADPH Oxidase 5 in Human Macrophages under Inflammatory Conditions: A Potential Mechanism of Reactive Oxygen Species Overproduction in Atherosclerosis. Oxid Med Cell Longev. Hindawi; 2019;2019:3201062-17.

35. Owens GK, Kumar MS, Wamhoff BR. Molecular Regulation of Vascular Smooth Muscle Cell Differentiation in Development and Disease. Physiol Rev. American Physiological Society; 2004 Jul 1;84(3):767-801.

36. Granger CB, Alexander JH, McMurray JJV, Lopes RD, Hylek EM, Hanna M, et al. Apixaban versus warfarin in patients with atrial fibrillation. N Engl J Med. 2011 Sep 15;365(11):981-92.

37. Connolly SJ, Ezekowitz MD, Yusuf S, Eikelboom J, Oldgren J, Parekh A, et al. Dabigatran versus warfarin in patients with atrial fibrillation. N Engl J Med. 2009 Sep 17;361(12):1139-51.

38. Schulman S, Kearon C, Kakkar AK, Mismetti P, Schellong S, Eriksson H, et al. Dabigatran versus warfarin in the treatment of acute venous thromboembolism. N Engl J Med. 2009 Dec 10;361(24):234252.

39. Patel MR, Mahaffey KW, Garg J, Pan G, Singer DE, Hacke W, et al. Rivaroxaban versus warfarin in nonvalvular atrial fibrillation. N Engl J Med. 2011 Sep 8;365(10):883-91.

40. Lee I-O, Kratz MT, Schirmer SH, Baumhäkel M, Böhm M. The effects of direct thrombin inhibition with dabigatran on plaque formation and endothelial function in apolipoprotein E-deficient mice. J Pharmacol Exp Ther. 2012 Nov;343(2):253-7.

41. Wagner N-M, Dressel T, Schafer K, Konstantinides S. Effect of the Factor Xa Inhibitor Rivaroxaban on Arterial Thrombosis in Wild-Type and Apolipoprotein E-Deficient Mice. Thromb Res. Elsevier Ltd; 2012 Nov 1;130(5):793-8.

42. Peeters F, Dudink E, Kimenai D, Weijs B, Altintas S, Heckman L, et al. Vitamin K Antagonists, NonVitamin K Antagonist Oral Anticoagulants, and Vascular Calcification in Patients with Atrial Fibrillation. TH Open. Georg Thieme Verlag KG; 2018 Oct 1;02(04):e391-8.

43. Chen B, Soto AG, Coronel LJ, Goss A, van Ryn J, Trejo J. Characterization of Thrombin-Bound Dabigatran Effects on Protease-Activated Receptor-1 Expression and Signaling In Vitro. Mol Pharmacol. 2015 May 1.

44. Raghavan S, Singh NK, Mani AM, Rao GN. Protease-activated receptor 1 inhibits cholesterol efflux and promotes atherogenesis via cullin 3-mediated degradation of the ABCA1 transporter. J Biol Chem. 2018 Jul 6;293(27):10574-89.

45. Rana R, Huang T, Koukos G, Fletcher EK, Turner SE, Shearer A, et al. Noncanonical Matrix Metalloprotease 1-Protease-Activated Receptor 1 Signaling Drives Progression of Atherosclerosis. Arterioscler Thromb Vasc Biol. 2018 Jun;38(6):1368-80.

46. Willis-Fox O, Preston RJS. Molecular basis of protease-activated receptor 1 signalling diversity. J Thromb Haemost. John Wiley \& Sons, Ltd (10.1111); 2019 Sep 24;3(9):1800. 


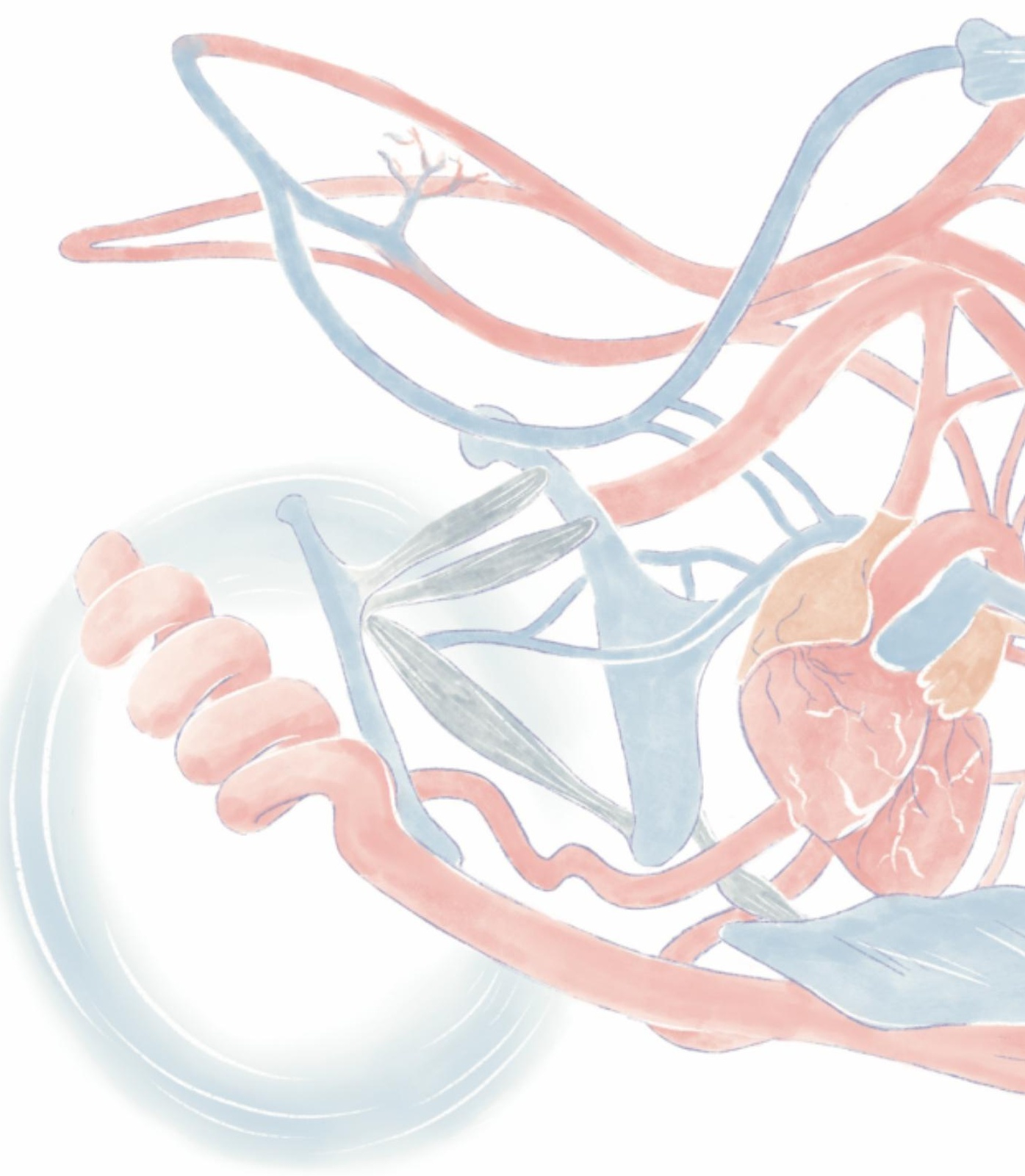




\title{
Addendum
}

\author{
Summary
}


In this thesis, the main focus of my work was to investigate the effects of (anti)coagulation on atherosclerosis development. Herein, we focussed on the role of the vascular smooth muscle cells (VSMC) as key-mediators of atherosclerosis.

In chapter 1 we describe the consequence of cardiovascular disease. Moreover, we elaborate on the role of vitamin $\mathrm{K}$ metabolism in coagulation and calcification, related to atherosclerosis. Atherosclerosis is a chronic inflammation of the vessel wall, mediated by lipid accumulation in the intimal space under endothelial cells. Atherosclerosis development is linked to endothelial cell activation, migration of inflammatory cells and proliferation of VSMC. Inflammation, but also apoptosis and extracellular vesicles are all linked to the initiation of vascular calcification. The location and size of calcification has an impact on the clinical consequences. I have focussed on intimal calcification and distinguished vascular calcification in micro- and macrocalcification. Moreover, I discuss calcification inhibitors and the effect of loss of inhibitory effect of vitamin $\mathrm{K}$ dependent matrix Gla protein upon vitamin $\mathrm{K}$ antagonist (VKA) treatment. In Chapter 2 we reviewed effects of oral anticoagulation treatment using VKA and direct non-vitamin $\mathrm{K}$ antagonists (NOAC) on coagulation and the vasculature. We briefly introduce the origin of oral anticoagulation treatment with VKA and continue with the comparison of anticoagulation between VKA and NOAC. We discuss in more detail the side effects of both forms of anticoagulation on the vessel wall. Here, we underline the role of vitamin $\mathrm{K}$ dependent proteins and protease activated receptor signalling on VSMC.

The clinical consequences of different forms of calcification in the vasculature is debated in chapter 3 were we further elucidated the interplay between coagulation and calcification. We show that the presence of vascular calcification promotes a systematic prethrombotic state, with a key role for VSMC phenotypic switching. Synthetic VSMC increase coagulation potential compared to contractile VSMC and calcification of VSMC further aggravates coagulation. The increased coagulation on calcified VSMC is predominantly occurring under flow conditions which increase fibrin formation compared to synthetic non-calcified VSMC. Additionally, we show that VSMC mediates coagulation predominantly via the extrinsic coagulation pathway. Our results indicate that calcified atherosclerotic plaques are more prone to induce thrombosis upon rupture. Additionally, our data support the association between presence of calcification and risk of cardiovascular events.

Currently, treatment for vascular calcification is lacking. Therefore, we investigated vitamin $\mathrm{K}$ (MK7) supplementation, after initial VKA treatment, on atherosclerosis development and intimal calcification. In chapter $\mathbf{4}$ we report that discontinuation of VKA treatment has beneficial effect on atherosclerosis. Discontinuing of VKA treatment attenuates atherosclerotic plaque calcification and progression. Moreover, vitamin K subtype MK7 supplementation improves atherosclerotic plaque environment by reducing both oxidative stress and fetuin-A uptake and increasing collagen content, likely via direct effects 
on VSMC. Our results support clinical testing of MK7 as treatment for atherosclerosis and calcification.

For decades, VKA is used as long-term anticoagulant treatment. Nevertheless, VKA have unfavourable pharmacokinetics, a small therapeutic window and requires frequent monitoring resulting in the development of direct non-vitamin $\mathrm{K}$ antagonist (NOAC). In chapter 5 we investigated effects of warfarin and dabigatran on the development of atherosclerosis, with a focus on the role of anticoagulation on calcification development. While VKA aggravated atherosclerosis and calcification, dabigatran reduced atherosclerotic plaque progression and had no significant effect on calcification development. Both VKA and thrombin increased VSMC oxidative stress and extracellular vesicle release, and thrombin mediated effects were attenuated by dabigatran. Our findings suggest that the choice of anticoagulant treatment for patients at risk for arterial and venous thrombosis influence atherosclerosis development, thereby altering the risk for future cardiovascular events.

VSMC phenotypic switching plays an important role in controlling vascular health. Repair of the vessel requires contractile VSMC to switch towards a synthetic phenotypical. After repair, differentiation back into contractile state is necessary to regulate vascular contractility. However, upon prolonged pathological stress phenotypic switching of VSMC plays a pivotal role in developing vascular disease. Therefore, we investigated the effect of VSMC phenotypical switching in chapter 6 and report the novel finding that Nox 5 acts as a novel regulator of VSMC phenotypic switching. VSMC switching promotes calcification via increased extracellular vesicle release. Extracellular vesicles take up extracellular calcium and form the nidus for calcification. Nox5 regulates VSMC phenotypic switching via increasing oxidative stress. Hence, inhibition of VSMC phenotype switching in cardiovascular disease may have therapeutic benefit by limiting the development of vascular calcification.

In conclusion, the data described in my thesis provide further insight in the development and clinical consequences of vascular calcification. Both VKA and NOAC are potent anticoagulation treatment, in which VKA aggravates and NOAC dabigatran improves atherosclerosis development. Nox 5 may serve as a novel target for controlling cardiovascular disease while vitamin $\mathrm{K}$ supplementation may be used for limiting vascular calcification. 


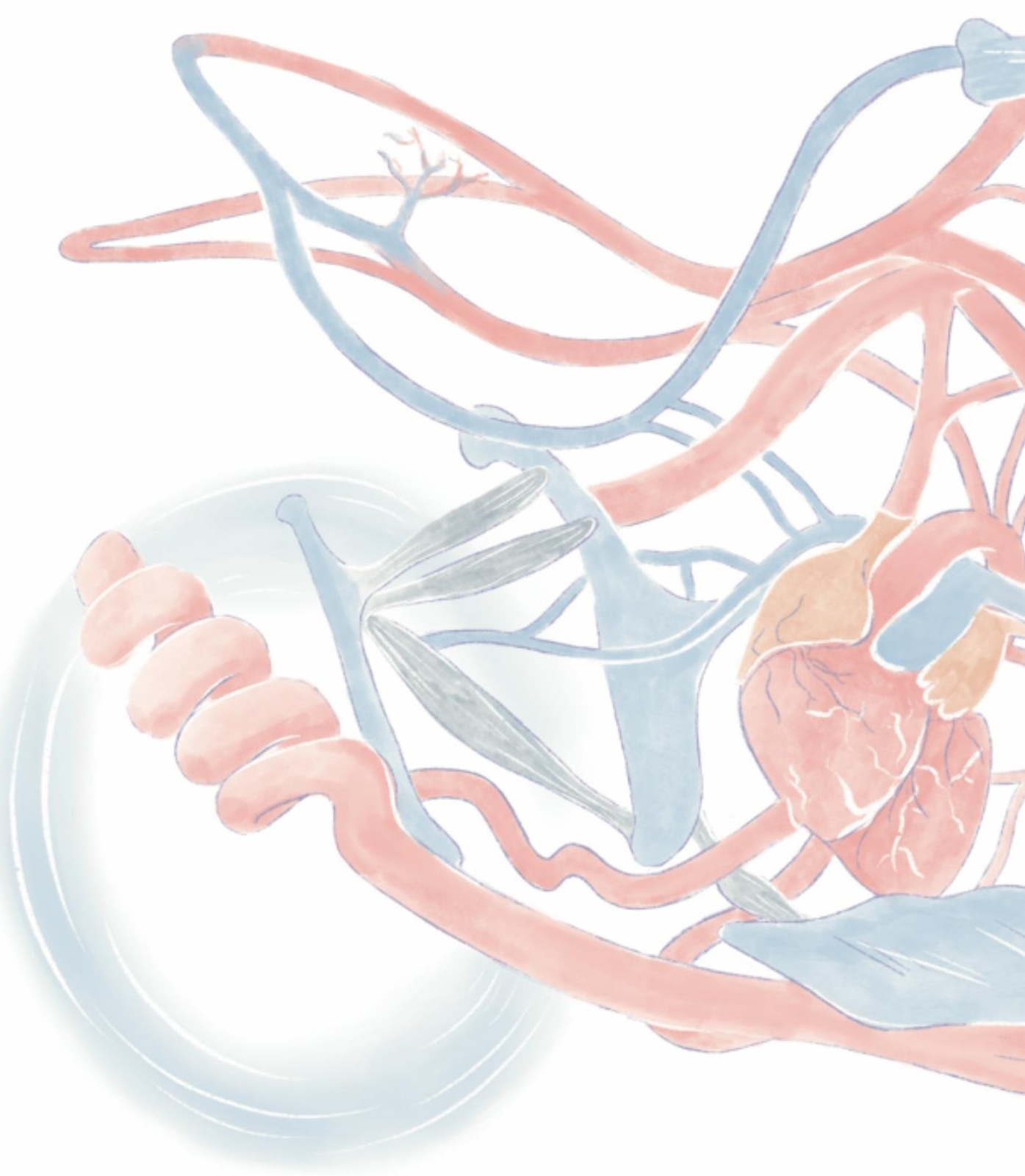




\section{Addendum}

Nederlandse samenvatting 
In dit proefschrift ligt de focus van mijn onderzoek op de effecten van (anti)stolling op de ontwikkeling van atherosclerose, waarin we specifiek kijken naar de rol van de gladde spiercel.

In hoofdstuk 1 worden de gevolgen van cardiovasculaire aandoeningen uitgelicht en wordt de invloed van stolling en vasculaire calcificatie besproken. Daarnaast gaan we uitgebreider in op rol van het vitamine $\mathrm{K}$ metabolisme in de stolling en calcificatie van de atherosclerotische plaque. Atherosclerose is een chronisch ontstekingsproces in de vaatwand dat veroorzaakt wordt door opstapeling van lipiden in de intimale ruimte onder de endotheelcellen. De ontwikkeling van atherosclerose is geassocieerd aan de activatie van endotheelcellen, migratie van ontstekingscellen en proliferatie van gladde spiercellen. Ontsteking, maar ook apoptose en extracellulair vesikles zijn verbonden aan het ontstaan van vasculaire calcificatie. De locatie en grootte van vasculaire calcificatie voorschrijft klinische gevolgen. Ik heb gefocust op intimale calcificatie en onderscheid gemaakt tussen micro- en macrocalcificatie. We gaan in op calcificatie inhibitoren en het verlies van de vitamine $\mathrm{K}$ afhankelijke calcificatie inhibitor matrix Gla protein als gevolg van vitamine $\mathrm{K}$ antagonist (VKA) behandeling. In Hoofdstuk 2 hebben we de effecten van orale antistolling behandeling door VKA en directe niet vitamine $\mathrm{K}$ antagonist (NOAC) op zowel antistollingsgebied als het vaatstelsel gereviewd. We omschrijven kort de ontdekking van orale antistolling behandeling met VKA en vergelijken daarna VKA en NOAC op het gebied van stolling. Bovendien wordt de rol van beide antistollingsmedicijnen op het vaatstelsel in meer detail besproken. Hierbij ligt de focus op de rol van vitamine $\mathrm{K}$ afhankelijke eiwitten en protease geactiveerde receptor signalering in de gladde spiercel.

De klinische gevolgen van verschillende vormen van vasculaire calcificatie wordt betwijfeld in hoofdstuk 3 waar we de interactie tussen stolling en calcificatie verder ontrafelen. We laten zien dat de aanwezigheid van calcificatie resulteert in een systematische pre trombotische staat. Een belangrijke rol in het induceren van de pre thrombotische staat is weggelegd voor de fenotypische verandering van gladde spiercellen. Synthetische gladde spiercellen verhogen het stollingspotentieel ten opzichte van contractiele gladde spiercellen. Het stollingspotentieel is verder verhoogd door calcificatie van gladde spiercellen. Deze gevolgen van fenotypische veranderingen is extra benadrukt door blootstelling van gladde spiercellen aan stromend bloed. Hier verhoogd de gecalcificeerde gladde spiercel de fibrine formatie in vergelijking met de niet gecalcificeerde gladde spiercellen. Daarnaast laten we zien dat gladde spiercellen de stolling vooral mediëren via de extrinsieke stolling route. Deze resultaten wijzen erop dat een gecalcificeerde plaque vatbaar is voor trombosevorming als deze scheurt. Daarnaast ondersteunen onze resultaten de connectie tussen de aanwezigheid van calcificatie en het risico op een cardiovasculair evenement.

Momenteel is er geen behandeling voor vasculaire calcificatie. Daarom hebben we de effecten vitamine K (MK7), na een eerste periode van VKA-behandeling, op de ontwikkeling van atherosclerose en intimale calcificatie onderzocht. In hoofdstuk 4 laten we de positieve effecten van het stoppen met een VKA-behandeling op atherosclerose zien. Daarnaast 
verbeterde MK7 supplementatie de atherosclerotische plaque door het verlagen van zowel oxidatieve stress als fetuin-A opnamen en het verhogen van de hoeveelheid collageen in de atherosclerotische plaque. Dit zijn waarschijnlijk gevolgen van directe effecten van MK7 op de gladde spiercel. Onze resultaten onderbouwen de gedachte om MK7 in de kliniek te testen voor behandeling van atherosclerose en calcificatie.

Voor tientallen jaren zijn VKA gebruikt als langdurige orale antistollingsbehandeling. Door de ongunstige farmacokinetiek, kleine therapeutische ruimte en regelmatige controle zijn er alternatieve orale antistolling medicijnen ontwikkeld genaamd directe geen vitamine $\mathrm{K}$ antagonist oftewel NOAC. In hoofdstuk 5 onderzochten we de gevolgen van VKA, warfarine, en NOAC, dabigatran etexilate, op de ontwikkeling van atherosclerotische plaques. We focusten specifiek op de rol van antistolling behandeling in de ontwikkeling van vasculaire calcificatie. VKA-behandelding verergerde de ontwikkeling van atherosclerose en calcificatie, terwijl dabigatran de ontwikkeling van atherosclerose verminderde en geen effect had op de ontwikkeling van calcificatie. Zowel VKA als trombine verhoogde oxidatieve stress en extracellulaire vesikels in de gladde spiercel, maar de effecten van trombine werden verminderd door toevoeging van dabigatran. Onze bevindingen suggereren dat de keuze voor antistolling medicijn, voor patiënten met een risico op een arteriële en veneuze trombose, de ontwikkeling van atherosclerose beïnvloed en daarmee het risico op een toekomstig cardiovasculair event.

Fenotypische verandering van gladde spiercellen speelt een belangrijke rol in het controleren van vasculaire gezondheid. Voor het repareren van de vaatwand moet de contractiele gladde spiercel veranderen in een synthetische gladde spiercel. Na de reparatie is het belangrijk dat de synthetische spiercel terug veranderd in een contractiele gladde spiercel voor het reguleren van vasculaire contractiliteit. Echter, na langdurige pathologische stress speelt het fenotypisch veranderen van de gladde spiercel een cruciale rol in de ontwikkeling van pathologische vasculaire aandoeningen. Daarom hebben we in hoofdstuk $\mathbf{6}$ de gevolgen van fenotypische verandering in de gladde spiercel onderzocht en beschrijven de nieuwe bevinding dat deze gereguleerd wordt door Nox5. Het fenotypische veranderen van de gladde spiercel bevordert de ontwikkeling van calcificatie via extracellulaire vesikles. Extracellulaire vesikles nemen extracellulaire calcium op en vormen zo het startpunt voor de ontwikkeling van calcificatie. Nox5 controleert de fenotypische verandering van gladde spiercellen door middel van oxidatieve stress. Dus, het blokkeren van fenotypische verandering van de gladde spiercel bij een pathologische aandoening kan resulteren in een positief effect op de ontwikkeling van vasculaire calcificatie.

Tot slot, de resultaten beschreven in dit proefschrift zorgen voor nieuwe inzichten in de ontwikkeling en klinische gevolgen van vasculaire calcificatie. Zowel VKA als NOAC zijn goede antistollingsmedicijnen, maar waar VKA de ontwikkeling van atherosclerose verergert wordt deze juist verbeterd door NOAC. Daarnaast kan Nox5 geütiliseerd worden als nieuw doelwit om cardiovasculaire aandoeningen te controleren en kan vitamine $\mathrm{K}$ supplementatie zorgen voor het limiteren van calcificatie. 


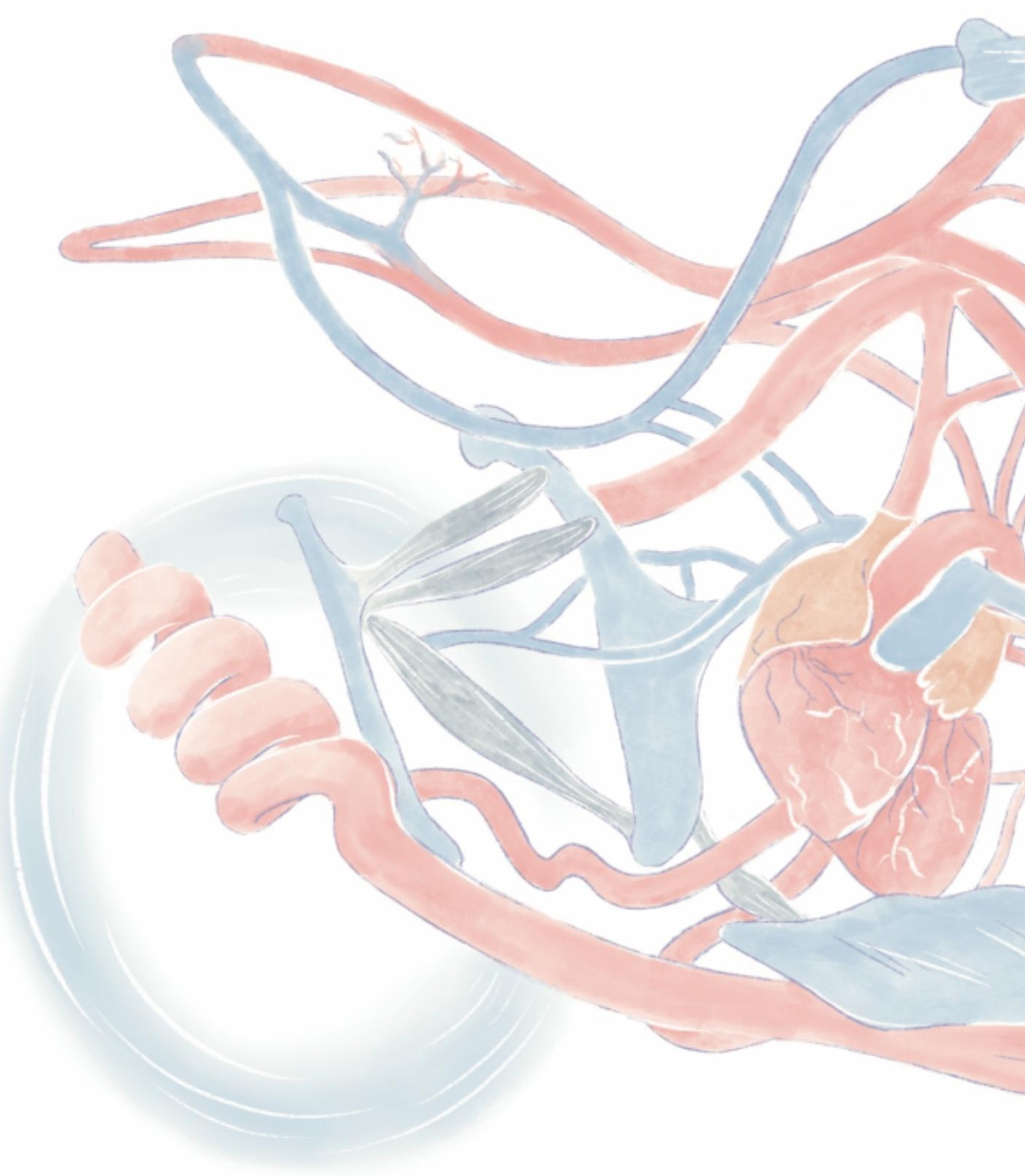




\title{
Addendum
}

\author{
Valorisation
}


In this chapter I discuss possible opportunities for valorisation of knowledge based on the findings described in this thesis. Valorisation is about the impact created by the transfer of scientific knowledge. This is not limited to money, but can also result in spin-off companies, patents or updates in medial guidelines. Research can be split into basic and clinical research. Here, we present translational research with focus on increasing basic knowledge to have a direct impact on medical care. Knowledge obtained via basic translational research by providing mechanistic insight, clinical research can be better and more specifically conducted. This interplay is crucial for further improving our medical care.

The main objective of this thesis is to gain further insight in the role of anticoagulants and vitamin $\mathrm{K}$ metabolism in cardiovascular disease, focusing on the role of vascular smooth muscle cells in this process.

Vascular calcification was long thought to be a passive process, but is now accepted as an active and highly regulated process. Vascular calcification is acknowledged as independent risk factor for cardiovascular disease and can be related to several leading cardiovascular disease resulting in death in the western world. The impact of anticoagulant treatment on calcification and cardiovascular disease is discussed in more detail in chapter

\section{1 and 2.}

In Chapter 3 I have investigated the interplay between intimal calcification and coagulation. The consequence of intimal calcification is currently debated. It is currently accepted that microcalcification, or spotty calcification, worsens plaque stability and thereby increase the chance of cardiovascular risk. On the other hand, macrocalcification, or sheetlike calcification has been put forward as being beneficial and aggravating atherosclerosis. Moreover, volume and density of calcification influence cardiovascular outcome differently. Our research demonstrates that intimal calcification activates the coagulation system. Increased coagulation has been shown to worsen atherosclerosis and thereby increases cardiovascular risk. Thus, our research indicates that VKA-induced vascular calcification worsens cardiovascular outcome. These results give further insight in the role of vascular calcification in cardiovascular disease risk and encourages to investigate ways to reduce vascular calcification.

In Chapter 4, we investigated vitamin K supplementation as potential treatment option for intimal calcification. Currently, treatment to prevent, hold or regress vascular calcification is lacking. We identified beneficial effects of vitamin $\mathrm{K}$ on atherosclerosis development, likely mediated via VSMC. Moreover, we show that discontinuation of VKA treatment has beneficial outcome on atherosclerosis. Taken together, we demonstrate the importance of vitamin $\mathrm{K}$ for vascular health. Biomarkers of vitamin $\mathrm{K}$ status, such as vitamin $\mathrm{K}$ dependent proteins are currently being used to predict vascular calcification. The role of vitamin $\mathrm{K}$ in vascular disease has further led to the collaboration with companies selling vitamin K2 (Nattopharma ASA) and companies measuring vitamin K status using the vitamin $\mathrm{K}$ dependent matrix Gla-protein (IDS). 
We demonstrate in Chapter 5 that every anticoagulation drug has off-target effects thereby influencing cardiovascular disease differently. We compared the VKA warfarin and the NOAC dabigatran on atherogenesis. Our data suggest that patients with increased risk for arterial disease, i.e. vascular calcification, would benefit from NOACs. If translatable to the human situation, our data urge for a personalized choice of anticoagulant agent and can further help evaluate clinical decisions on the use of either VKA or NOAC treatment. Although NOAC are more expensive in direct costs, cardiovascular risk reduction will be beneficial for the health care system on the long run. Some patient groups, including patients with mechanical valve replacement and patients with end stage chronic kidney disease, are still limited to VKA treatment. Patients with end-stage chronic kidney disease are prone to develop cardiovascular disease. Our data support the development of NOAC that are not limited by clearance via the kidney. Also, novel anticoagulation therapies focusing on upstream coagulation factors XI and XII are currently being investigated to induce anticoagulation but limit bleeding risk.

In Chapter 6 the focus lies on the role of VSMC in the initiation of vascular calcification. We show that phenotypic switching of VSMC is key in the initiation of vascular calcification. Moreover, we identified Nox5 as key-regulator of pathological VSMC oxidative stress which mediates this phenotypic switching. VSMC plays a pivotal role in many vascular diseases and therefore Nox 5 is an interesting novel therapeutic target. Recently, the WHO recognized Nox inhibitors as a new therapeutic class. Nox5 is the least studied of the Nox family, probably due its absence in rodents. The Nox inhibitor Setanaxib is recognized as Nox1/4 inhibitors, however no specific targets for Nox 5 are available yet.

Of note, the results described in my thesis are from experimental animal models and in vitro work on VSMC. Therefore, confirmation of findings in clinical trials will be important before implementation in clinical guidelines. Nevertheless, the underlying mechanistical insight creates the opportunity for novel therapeutic approaches and highlights the importance of personalized medicine. Moreover, the results will open up opportunities to limit progression of vascular calcification. 


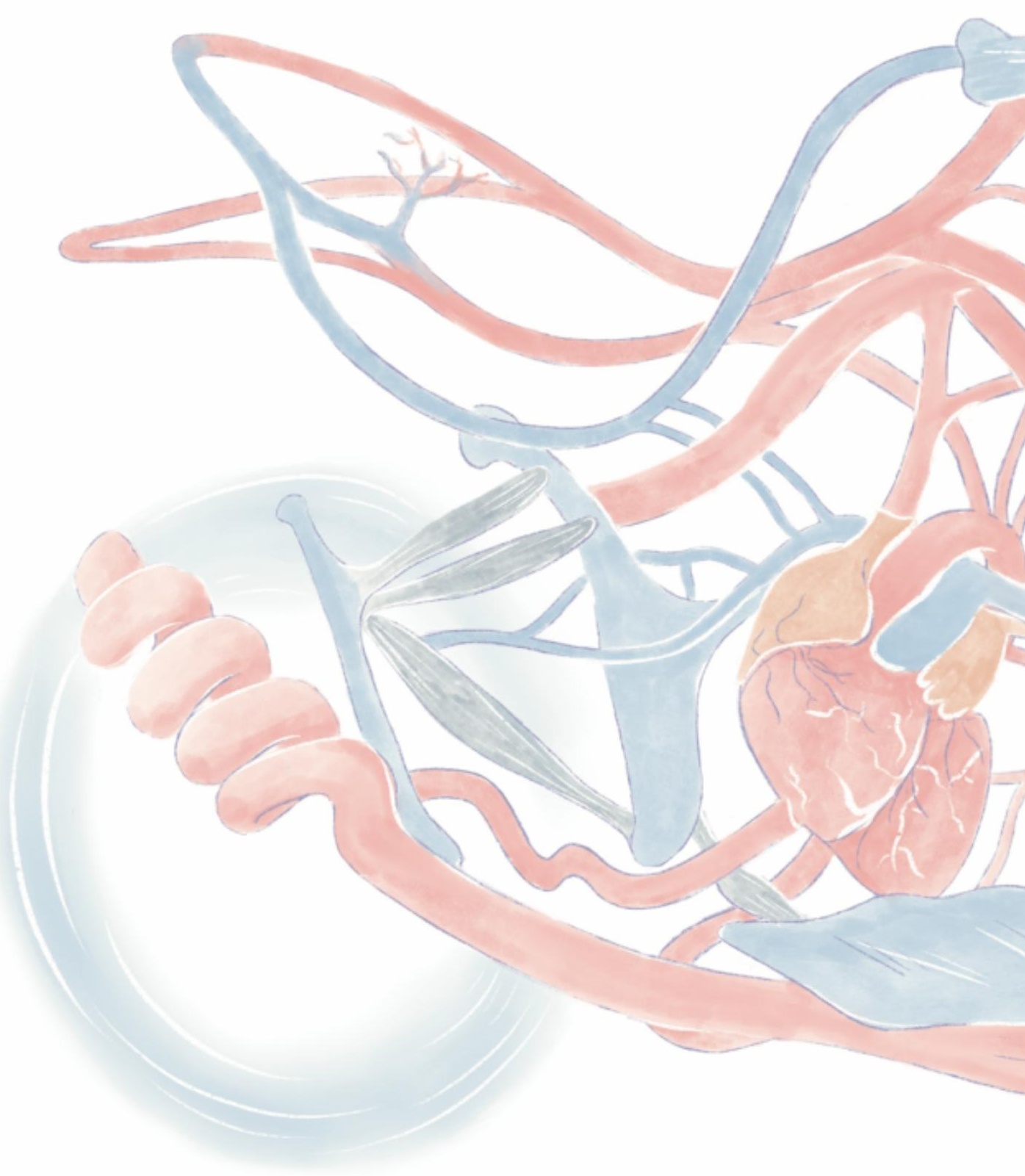




\title{
Addendum
}

\author{
About the author
}


Rick Henricus Petrus van Gorp was born on 3th of March in 1991 in Oosterhout, the Netherlands. In Oosterhout he graduated from the Havo completing a Nature and Health profile at Mgr. Frencken College in 2008.

After graduation of high school he started to study applied biological and medical laboratory at the University of Avans in Breda. Rick specialized in medical research and animal science and after a 9 months internship under the supervisor of Dr. Ramon Langen at Maastricht University, received his Bachelors degree in July 2012. After completion of the bachelors program he started a 2 year master program at University Maastricht focusing on biomedical science specializing in Clinical Molecular Sciences. In July 2014, Rick obtained his Masters degree after a 9 months internship under the supervision of Dr. Tim Wolfs at Maastricht University.

In September 2014, Rick started his industrial PhD program at Nattopharma ASA in collaboration with Maastricht University under the supervision of Prof. Dr. Leon Schurgers and Prof. Dr. Chris Reutelingsperger. The aim of his $\mathrm{PhD}$ project was to investigate the role of (anti)coagulation and vitamin K supplementation on vascular smooth muscle cells in the development of atherosclerosis development. 
ABOUT THE AUTHOR 


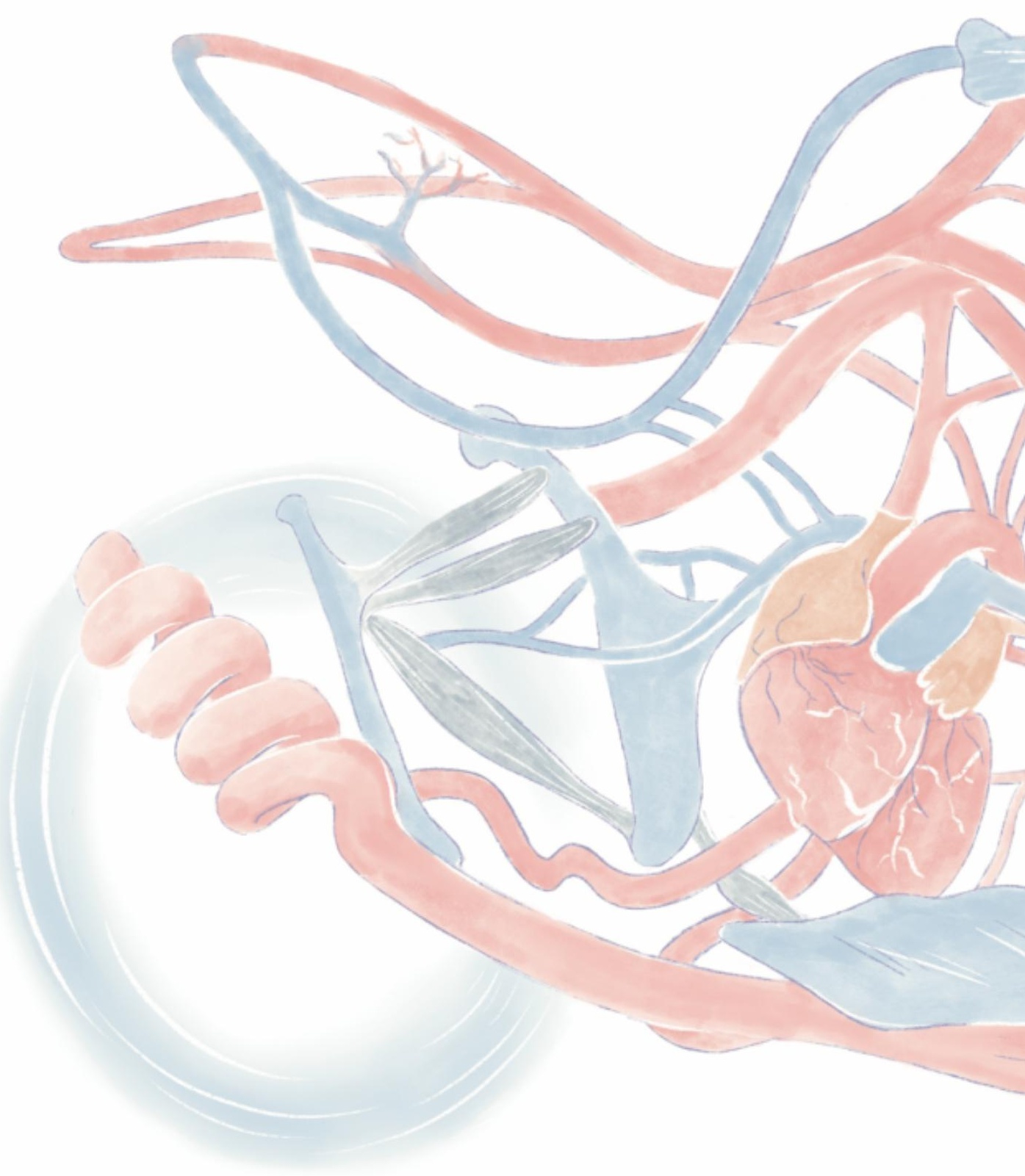




\title{
Addendum
}

\author{
Publications
}




\section{Publications}

Theeuwes WF, Pansters NAM, Gosker HR, Schols AMWJ, Verhees KJP, de Theije CC, van Gorp RHP, Kelders MCJM, Ronda O, Haegens A, Langen RCJ and Remels AHV. Recovery of muscle mass and muscle oxidative phenotype following disuse does not require GSK-3 inactivation. Biochim Biophys Acta Mol Basis Dis. 2020 Feb 19:165740. doi: 10.1016/j.bbadis.2020.165740.

Jelle J. posthuma, Jens J. N. posma, Rene van oerle, Peter Leenders, Rick H. van Gorp, Armand M. G. Jaminon, Nigel Mackman, Stefan Heitmeier, Leon J. Schurgers, Hugo ten Cate \& Henri M. H. Spronk. Targeting Coagulation Factor Xa Promotes Regression of Advanced Atherosclerosis in Apolipoprotein-E Deficient Mice. Sci Rep. Nature Publishing Group; 2019 Mar 7;9(1):3909.

Spronk HMH, Padro T, Siland JE, Prochaska JH, Winters J, van der Wal AC, Posthuma JJ, Lowe G, d'Alessandro E, Wenzel P, Coenen DM, Reitsma PH, Ruf W, van Gorp RH, Koenen RR, Vajen T, Alshaikh NA, Wolberg AS, Macrae FL, Asquith N, Heemskerk J, Heinzmann A, Moorlag M, Mackman N, van der Meijden P, Meijers JCM, Heestermans M, Renné T, Dólleman S, Chayouâ W, Ariëns RAS, Baaten CC, Nagy M, Kuliopulos A, Posma JJ, Harrison P, Vries MJ, Crijns HJGM, Dudink EAMP, Buller HR, Henskens YMC, Själander A, Zwaveling S, Erküner O, Eikelboom JW, Gulpen A, Peeters FECM, Douxfils J, Olie RH, Baglin T, Leader A, Schotten U, Scaf B, van Beusekom HMM, Mosnier LO, van der Vorm L, Declerck P, Visser M, Dippel DWJ, Strijbis VJ, Pertiwi K, Ten Cate-Hoek AJ, Ten Cate H. Atherothrombosis and Thromboembolism: Position Paper from the Second Maastricht Consensus Conference on Thrombosis. 2018. pp. 229-50.

Stöhr R, Schurgers L, van Gorp R, Jaminon A, Marx N, Reutelingsperger C. Annexin A5 reduces early plaque formation in ApoE -/- mice. PLoS ONE. Public Library of Science; 2017;12(12):e0190229.

Hendrikx G, Hackeng TM, van Gorp R, Bauwens M, Schurgers LJ, Mottaghy FM, et al. Use of Cyclic Backbone NGR-Based SPECT to Increase Efficacy of Postmyocardial Infarction Angiogenesis Imaging. Contrast Media Mol Imaging. Hindawi; 2017;2017(1):8638549-9.

de Theije CC, Schols AMWJ, Lamers WH, Ceelen JJM, van Gorp RH, Hermans JJR, Köhler SE, Langen RCJ. Glucocorticoid receptor signaling impairs protein turnover regulation in hypoxia-induced muscle atrophy in male mice. Endocrinology. 2017 Oct 23.

Döring Y, Noels H, van der Vorst EPC, Neideck C, Egea V, Drechsler M, Mandl M, Pawig LB, Jansen Y, Schröder K, Bidzhekov K, Megens RTA, Theelen W, Klinkhammer BM, Boor P, Schurgers LJ, van Gorp RH, Ries C, Kusters PJH, van der Wal AC, Hackeng TM, Gäbel G, Brandes RP, Soehnlein O, Lutgens E, Vestweber D, Teupser D, Holdt LM, Rader DJ, 
Saleheen D, Weber C. Vascular CXCR4 Limits Atherosclerosis by Maintaining Arterial Integrity: Evidence from Mouse and Human Studies. Circulation. American Heart Association, Inc; 2017 Apr 27;:CIRCULATIONAHA.117.027646.

van Gorp RH, Schurgers LJ. New Insights into the Pros and Cons of the Clinical Use of Vitamin K Antagonists (VKAs) Versus Direct Oral Anticoagulants (DOACs). Nutrients. 2015;7(11):9538-57.

Nikiforou M, Kemp MW, van Gorp RH, Saito M, Newnham JP, Reynaert NL, Janssen LE, Jobe AH, Kallapur SG, Kramer BW, Wolfs TG. Selective IL-1 $\alpha$ exposure to the fetal gut, lung, and chorioamnion/skin causes intestinal inflammatory and developmental changes in fetal sheep. Lab Invest. 2016 Jan;96(1):69-80.

Hendrikx T, Jeurissen MLJ, van Gorp PJ, Gijbels MJ, Walenbergh SMA, Houben T, van Gorp R. Pöttgens CC, Stientra R, Netea MG, Hofker MH, Donnders MMPC, Shiri-Sverdlov $\mathrm{R}$. Bone marrow-specific caspase-1/11 deficiency inhibits atherosclerosis development in Ldlr(-/-) mice. FEBS J. 2015 Jun;282(12):2327-38.

Brandenburg VM, Schurgers LJ, Kaesler N, Püsche K, van Gorp RH, Leftheriotis G, Reinartz S, Koos R, Krüger T. Prevention of vasculopathy by vitamin K supplementation: Can we turn fiction into fact? Atherosclerosis. Elsevier Ltd; 2015 May 1;240(1):10-6.

\section{Publications (in preparation)}

van Gorp RH, Baaten CCFMJ, Peeters FECM, Leenders P, Crijns H, Heemskerk J, ReutelingspergerCP, Spronk HM, Schurgers LJ. Vitamin K antagonist induces calcification and atherosclerotic plaque progression resulting in increased hypercoagulability. (Submitted)

van Gorp RH, Petsophonsakul P, AM Jaminon, Leenders P, Jahnen-Dechent W, Reutelingsperger CP, Schurgers LJ. Vitamin K inhibits vascular smooth muscle cell induced calcification via reduced oxidative stress and extracellular vesicle secretion. (In preparation)

van Gorp RH, Dijkgraaf I, Bauwens M, Leenders P, Jennen D, Dweck MR, Bucerius J, Briedé JJ, van Ryn J, Brandenburg V, Mottaghy F, Spronk HMH, Reutelingsperger CP, Schurgers LJ. Off-target effects of oral anticoagulants - vascular effects of vitamin $\mathrm{K}$ antagonist and non-vitamin $\mathrm{K}$ antagonist oral anticoagulants. (Submitted)

Furmanik M, Chatrou M, van Gorp R, Akbulut A, Willems B, Schmidt H, van Eys G, Bochaton-Piallat M, Proudfoot D, Biessen E, Hedin U, Matic LP, Mees B, Shanahan C, Reutelingsperger C, Schurgers L. Reactive oxygen-forming Nox5 links vascular smooth muscle cell phenotypic switching and extracellular vesicle-mediated-vascular calcification. (Submitted)

Till Seime, Asim C. Akbulut, Urszula Rykaczewska, Rick H. van Gorp, Olivia J. Waring, Moritz Lindquist Liljeqvist, Eva Karlöf, Mariette Lengquist, Andrew J. Buckler, Malin 
Kronqvist, Jan H.N. Lindeman, Anton Razuvaev, Leon J. Schurgers, Ulf Hedin, Ljubica Matic. Proteoglycan 4 is a key factor in chondrogenic smooth muscle cell differentiation during vascular remodeling and intimal calcification. (Submitted)

\section{Oral presentations}

van Gorp R, Willems B, Scaf B, Spronk H, Reutelingsperger C, Schurgers L. Effect of dabigatran etexilate and warfarin on atherosclerotic plaque calcification in Apoe ${ }^{-/}$mice. Nederlands vereniging voor Thrombose en Hemostase (NvTH) 2015, Koudekerke, The Netherlands.

van Gorp R, Jaminon A, Spronk H and Schurgers L. Effects of Dabigatran-etexilate and Warfarin on atherosclerotic plaque vulnerability. European Congress on Thrombosis and Haemostasis (ECTH) 2016, The Hague, The Netherlands

van Gorp RH, Jaminon A, Bauwens M, Bucerius J, Brandenburg V, Jahnen-Dechent W, Spronk HMH, Reutelingsperger CP and Schurgers LJ. Oral anticoagulants as modulators of arterial calcification. Nederlands vereniging voor Thrombose en Hemostase (NvTH) April 2017, Koudekerke, The Netherlands. 
PUBLICATIONS 


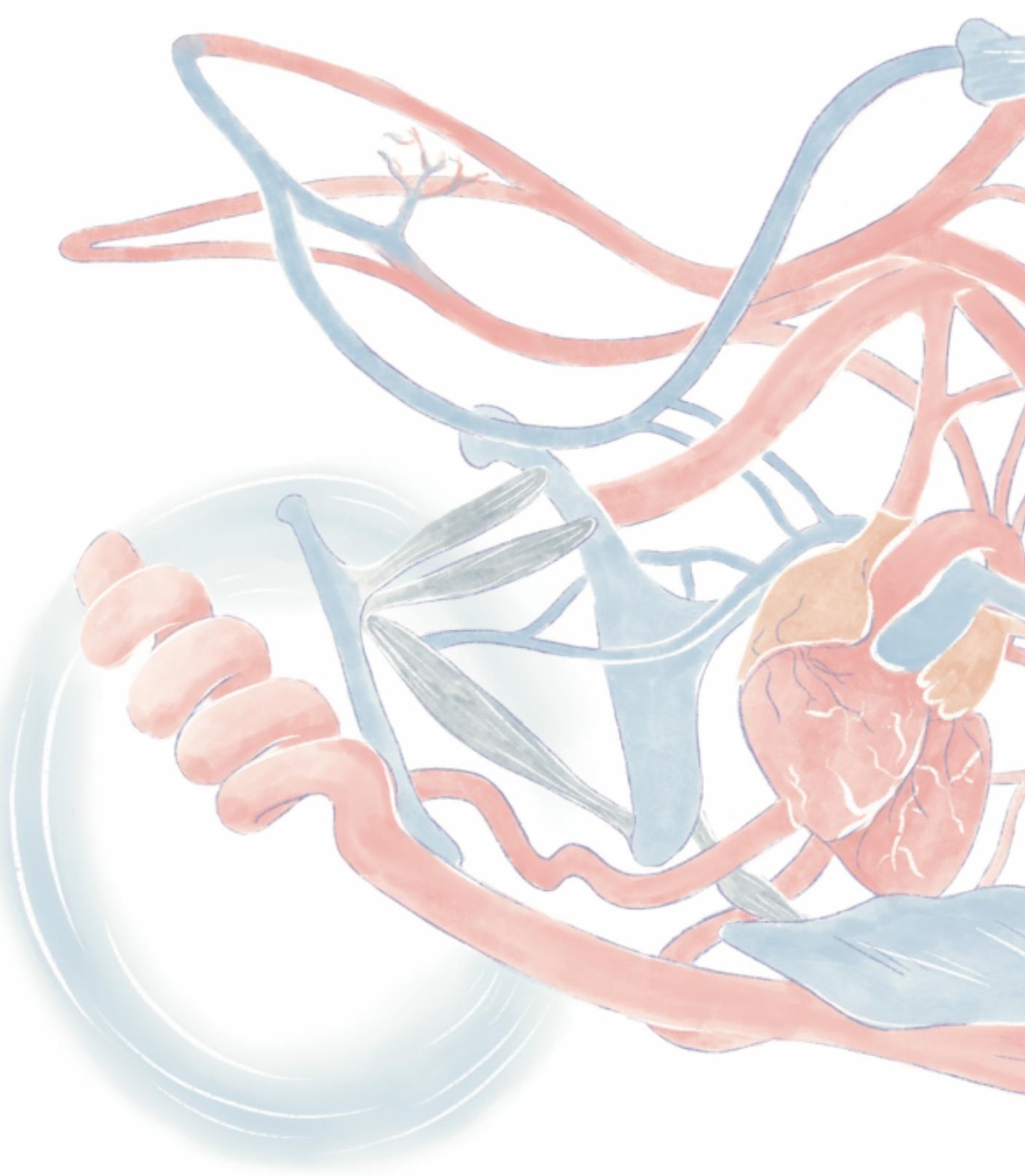




\title{
Addendum
}

\author{
Dankwoord
}


Met het dankwoord komen we op het einde van een lange, maar zeker ook een gezellige en leerzame weg. Er zijn vele mensen die ik wil bedanken voor alle hulp, steun maar ook voor de gezelligheid.

Mijn promotor, Prof. Dr. Schurgers. Beste Leon, allereerst wil ik je bedanken voor alle kansen die je me hebt gegeven. Ik kan me onze eerste ontmoeting tijdens de sollicitaties nog goed herinneren. Hierbij viel meteen je enorme enthousiasme op welke gedurende mijn hele PhD-traject vol aanwezig was. Deze enthousiasme was altijd een goede drijfveer en resulterende meer dan eens in een neven project. Ik heb altijd erg genoten van leerzame maar zeker ook gezellige reizen richting een congres of Nattopharma waar natuurlijke ook de benodigde versnaperingen bij aanwezig waren. Ik ben dankbaar dat je deur altijd openstond, wat dan weer wel de keerzijde had dat iedereen zomaar binnen viel in een meeting. Ik wil je enorm bedanken voor alle wijzen lessen die ik geleerd heb, zowel werk gerelateerd als privé. Ik wil je ook succes wensen met de enorme groei van jouw onderzoeksgroep de laatste jaren, waarvan ik weet dat het met jou enthousiasme alleen maar een succes kan worden.

Mijn promotor, Prof. Dr. Reutelingsperger. Beste Chris, je stond wat verder af van mijn onderzoek wat niks heeft afgedaan aan je bijdrage aan dit proefschrift. Je kritische opmerkingen, creatieve blik en onuitputtelijke bron van kennis hebben dit proefschrift naar een hoger niveau gebracht. Ik heb erg genoten van de congressen die we gezamenlijk bezocht hebben en waar we elkaar beter hebben leren kennen. Ik wil je graag bedanken voor alles wat je mij hebt geleerd en de kansen die je mij hebt gegeven.

Ik wil ook graag de beoordelingscommissie bedanken, Prof. dr. Kroon, Prof. dr. Middeldorp, Prof. dr. Post, Prof. dr. Biessen, en Dr. Boersma voor de tijd en moeite om mijn proefschrift te beoordelen.

Mijn paranimfen, Armand en Richard, voor beide zijn woorden niet genoeg. Beste Armand, we zijn tegelijk begonnen aan onze $\mathrm{PhD}$ ervaring en hebben daarmee het gehele project zij aan zij doorgelopen. Gedurende deze 5 jaar hebben wel veel samen mee gemaakt, zowel de ups en downs. Ik heb erg genoten van de congressen die we samen bezocht hebben, met als toppunt uiteraard het uitzicht in Boston, maar ook de talloze andere biertjes die we samen hebben gedeeld. Je bent altijd bereid andere te helpen waardoor we samen ook genoeg tijd in het lab hebben doorgemaakt. Ik wil je dan ook succes wensen met de laatste loodjes van je PhD en wens jou samen met Famke en Marit alvast een prachtige toekomst toe.

Beste Richard, we "kennen" elkaar van Avans en dat was genoeg. Ik heb erg genoten van onze talloze burger en bier avonden waarin jij al mijn verhalen hebt mogen aanhoren onder het genot van een biertje. Zowel onze wetenschappelijke discussie als de discussies die eigenlijk nergens op sloegen waren de relax momenten die ik soms even nodig had. Hiermee komt uiteraard de vraag: wanneer is de volgende? Je bent nu zelf aan je PhD bezig 
en ik wil je alvast al het beste toewensen met de afronding waarvan ik overtuigd ben dat dit goed komt.

I would like to thank Nattopharma for giving me the opportunity to do my industrial $\mathrm{PhD}$ in a collaboration between Nattopharma and Maastricht University. Herein I specially want to thank Henning, Laura, Frode, Hogne, Anne, Daniel and Kjetil for all your help, guidance and support.

I would like to thank Prof. dr. Paul Shiels for giving me the opportunity to join your group in Glasgow.

De collega's waarmee je misschien wel het grootste deel van je tijd mee door brengt, de kamergenoten. Dr. Willemse, Brecht, terwijl jij al in je laatste jaar zat begon ik net aan mijn $\mathrm{PhD}$. Jij hebt mij wegwijs gemaakt in het lab en was de vraagbaak gedurende mijn eerste periode. Daarnaast was het ook gewoon een gezellige tijd! Succes met je toekomstige carrière. Dear Ploi, you are also entering the last phase of your $\mathrm{PhD}$. When you arrived everyone felt sorry that you had to join the 2 guys, but you stood your ground and I hope you enjoyed it just as much as me! I enjoyed our discussions and really admire your always present smile. You have a good work life balance, which I feel grateful you put at pause to show us around in Chiang Mai where you even complained we had to little time, again thank you! I wish you all the best with finishing your PhD.

Beste Cecile, ik wil je graag bedanken voor alle hulp met de vele kleuringen die we hebben uitgevoerd. Daarin zitten uiteraard ook voor de vele leuke gesprekken en de avonturen op het lab en op de kamers. Beste Petra, hoewel jouw bijdrage bijna onzichtbaar lijkt zou dit proefschrift zonder jou niet bestaan. Naast al je hulp bij het administratieve gedeelte en experimenten heb ik genoten van onze gesprekken. Beste Niko, ik kan na deze jaren nog steeds dubbel liggen bij het gebruik van sommige vlaamse woorden tijdens de pauzes. Ik heb erg genoten van zowel de vele biertjes samen als het squashen met daaropvolgende sushi. Beste Peter, onze dieren expert. Het was altijd flink doorwerken, maar ook erg leuke en gezellige dagen. Beste Liset, je stond wat verder af van mijn onderzoek, desondanks stond je er altijd open voor om mijn vragen te beantwoorden als ik weer iets nieuws ging proberen.

Jullie zijn met zijn allen cruciaal voor zowel de geweldige sfeer op het lab als het draaiende hiervan houden. Off course I also want to thank the rest of the Reutelingsperger/Schurgers group for the entertaining time on the lab, fun day outs and relaxing breaks.

Naast onze groep wil ik ook de afdeling Biochemie bedanken voor alle leuke uitjes en gezelligheid gedurende mijn $\mathrm{PhD}$-traject. Hierin wil ik graag een paar mensen eruit lichten: Annemiek, Constance, Danique, Daniëlle, Jens, Joram, Peter en Stijn. Jullie hebben een 
extra belangrijke rol gespeeld in mijn PhD-traject, bedankt! Daarnaast wil ik ook Trees en Lidewij bedanken voor de administratieve hulp en de leuke gesprekken.

Naast collega's wil ik ook mijn vrienden bedanken voor hun steun en de afleiding die ze mij hebben gegeven tijdens mijn PhD-traject. Hierbij hebben jullie 1 van de waardevolste bijdrage geleverd, bedankt! Ook wil ik graag mijn familie bedanken welke altijd interesse hebben getoond in mijn project, dit heeft me altijd gemotiveerd.

Lieve schoonfamilie, ik wil jullie graag bedanken voor alle steun. In het bijzonder Lucie voor het maken van de prachtige cover!

Lieve mam en pap, zonder jullie zou dit proefschrift niet aanwezig. Jullie hebben me elk moment in mijn leven gesteund. Jullie hebben mij ook altijd mijn eigen weg laten zoeken en staan altijd op de zijlijn klaar als er iets is. Ik kan mezelf geen andere ouders wensen. Dit dankjewel staat in het niets met hoe dankbaar ik werkelijk ben maar toch, bedankt voor alles! Laura, lieve grote, kleine zus, ook jou wil ik graag bedanken voor alle steun en dat je altijd voor mij klaar staat!

Lieve José, woorden schieten te kort om te vertellen hoe dankbaar ik je ben. Je steun en geduld als ik er even klaar mee was, maar ook de ontspanning als ik het even los moest laten. Mijn habibi, ik kijk uit naar onze toekomst samen!

Om het samen te vatten: Bedankt!! 